\title{
Characterization and Control of Wave Propagation in the Heart
}

\author{
Dissertation \\ For THE AWARD OF THE DEGREE \\ Doctor rerum naturalium \\ of the University of Göttingen \\ within the doctoral program \\ Göttingen Graduate School for Neurosciences, Biophysics, and \\ Molecular Biosciences (GGNB) \\ of the Georg-August University School of Science (GAUSS)
}

\author{
submitted by \\ Sebastian Stephan Berg \\ from Bielefeld
}

Göttingen, 2018 


\section{Thesis Committee}

Prof. Dr. Stefan Luther, Institut für Nichtlineare Dynamik, Georg-August-Universität Göttingen

Prof. Dr. Tim SAlditT, Institut für Röntgenphysik, Georg-August-Universität Göttingen

Prof. Dr. Martin GöPfert, Institut für Zelluläre Neurobiologie, Georg-August-Universität Göttingen

\section{Members of the Examination Board}

Referee: Prof. Dr. Stefan Luther, Institut für Nichtlineare Dynamik, Georg-August-Universität Göttingen

$2^{\text {nd }}$ Referee: Prof. DR. Tim SAlditT, Institut für Röntgenphysik, Georg-August-Universität Göttingen

\section{Further members of the Examination Board:}

Dr. Karen Alim, Biological Physics and Morphogenisis, Max-Planck-Institut für Dynamik und Selbstorganisation

Prof. Dr. Wolfram Kollatschny, Institut für Astrophysik, Georg-August-Universität Göttingen 


\section{Table of contents}

Glossary and Abbreviations vi

1 Introduction $\quad 1$

1.1 The Heart . . . . . . . . . . . . . . . . . . . . . . . . . . . 3

1.1.1 Electrical Conductance . . . . . . . . . . . . . . . 4

1.1.2 The Cardiomyocyte and Action Potential . . . . . . . . . 5

1.1.3 Basic Mechanisms and Modelling of the Cardiac Cell . . . . . . 6

1.2 Excitable Media . . . . . . . . . . . . . . . . 7

1.2.1 Local Dynamics to Spatial Organization . . . . . . . . . . . 9

1.3 Cardiac Arrhythmia . . . . . . . . . . . . . . . . . . . . . 11

1.4 Spiral Wave Breakup . . . . . . . . . . . . . . . . . . . 12

1.5 Effect of External Electric Fields . . . . . . . . . . . . . . . . . . . . 13

1.6 Ischemia . . . . . . . . . . . . . . . . . . . . . . . . 14

2 Experimental Methods $\quad \mathbf{1 5}$

2.1 Langendorff Perfusion . . . . . . . . . . . . . . . . . . . 15

2.1.1 Contraction Uncoupling . . . . . . . . . . . . . . . . 17

2.2 Optical Mapping . . . . . . . . . . . . . . . . . . . . 18

2.2.1 Limitations of Optical Mapping . . . . . . . . . . . . . . . . 19

2.3 Further Measurements . . . . . . . . . . . . . . . . . . . . . 20

2.4 Far Field Shocks Protocols and Local Pacing . . . . . . . . . . . . . 20

2.5 Software and Experimental Control . . . . . . . . . . . . . . . . . . . . . 21

2.6 Induction and Termination of VF . . . . . . . . . . . . . . . . . . . . . . . . . . . . . .

2.6.1 Induction Protocol . . . . . . . . . . . . . . . 21

2.6.2 Defibrillation ..................... 22

3 Theoretical Background $\quad 23$

3.1 Activation Maps and Time . . . . . . . . . . . . . . . . 23

3.1.1 Theoretical Foundation of Wave Emission at Boundaries . . . . 23

3.1.2 From Circular Boundaries to Activation Times . . . . . . . . . 24

3.1 .3 Typically Activated Areas . . . . . . . . . . . . . . . . . . 25

3.2 Defect Mediated Turbulence and Markov Chain Modelling . . . . . . . 26

3.2.1 Markov Chain Models of the Number of PS . . . . . . . . . 26

3.2.2 Properties and Interpretation of the Transition Rates . . . . . . 29

3.2.3 Properties and Interpretation of the Stationary Distribution . . 31 
4 Video Processing and Data Analysis Methods 33

4.1 Preprocessing of the Video Data . . . . . . . . . . . . . . . . . . 35

4.1.1 Manual Creation of Masks . . . . . . . . . . . . . . . . . 35

4.1.2 Basic Video Preprocessing . . . . . . . . . . . . . . . . 35

4.1.3 Spatial Smoothing . . . . . . . . . . . . . . . 36

4.1.4 Rescaling to the Fractional Fluorescent . . . . . . . . . . . . . . 36

4.2 Detection of Activation Times . . . . . . . . . . . . . . 36

4.3 Further Analysis . . . . . . . . . . . . . . . . . . . . . . . . . . . . . 39

4.3.1 Activation Map and Time Analysis for Quiescent Tissue . . . . 39

4.3 .2 Cycle Length . . . . . . . . . . . . . . . . . . . . 40

4.3.3 Action Potential Duration . . . . . . . . . . . . . . . . 40

4.3.4 Triangularity Index . . . . . . . . . . . . . . . . . . . . 41

4.4 Phase Singularity Identification and Tracking . . . . . . . . . . . . . . 42

4.4.1 Overview ........................ . . 43

4.4.2 Typical Definitions for the Phase . . . . . . . . . . . . . . 43

4.5 Problems and Limitations of Singularity Identification . . . . . . . . . 45

4.5.1 Complex Propagation Patterns . . . . . . . . . . . . . 45

4.5.2 Minimum Propagation Speed . . . . . . . . . . . . . . . 45

4.5.3 Definition of the Phase in this Thesis . . . . . . . . . . . . 46

4.5.4 Identification of Wavefronts and Phase Singularities . . . . . . . 46

4.5.5 Filtering of Short Lived Phase Singularities . . . . . . . . . . . 48

4.5.6 Estimating Creation, Annihilation, Entering, and Leaving Rates 49

4.5.7 Limitations of PS Identification and Tracking . . . . . . . . . 50

4.6 Notes on Images and Plots . . . . . . . . . . . . . . . . . . . . . 50

5 Ischemia Characterization using Pinacidil $\quad 51$

5.1 Pinacidil as Ischemia Model . . . . . . . . . . . . . . . . . . 51

5.1 .1 Effects of Pinacidil . . . . . . . . . . . . . . . 52

5.2 Experimental Design . . . . . . . . . . . . . . . . . . . 52

5.2 .1 List of Experiments . . . . . . . . . . . . . . . 54

5.3 General Analysis Notes . . . . . . . . . . . . . . . . . . 54

5.4 Results . . . . . . . . . . . . . . . . . 55

5.4.1 Control of APD as a Marker for Pinacidil Effect . . . . . . . . 55

5.5 Activation Time Analysis Results . . . . . . . . . . . . . 56

5.5.1 Activation Time Overview for the Different Experiments . . . . 58

5.5.2 Dependency on the Pinacidil Level . . . . . . . . . . . . . . 58

5.6 Results Characterizing the Arrhythmia . . . . . . . . . . . . . . 59

5.7 Summary . . . . . . . . . . . . . . . . . 62

6 Analysis of the PS Markov Model and its Parameters 63

6.1 NPS Rates of Change . . . . . . . . . . . . . . . . 63

6.1.1 Results from the Pair Creation Rate . . . . . . . . . . . 65

6.1.2 Results from the Pair Annihilation Rate and Pair Statistics . . 68

6.2 Results for the Probability Distribution of the NPS . . . . . . . . . 69 
6.2.1 Relation to Self Termination . . . . . . . . . . . . . . . 73

6.3 Interpretation of Markov Model Results . . . . . . . . . . . . . . . . . 74

6.4 Autocorrelation Function of the NPS . . . . . . . . . . . . . 76

6.5 Nonstationarity and Intermittence . . . . . . . . . . . . . . . . 78

7 Spatial Phase Singularity Organization and Emergent Dynamics 83

7.1 Emergent Dynamics of Spatio-Temporal Chaos in a Heterogeneous Ex-

citable Medium . . . . . . . . . . . . . . . . . . 84

7.2 Experimental Findings of Spatial Structuring . . . . . . . . . . . . . 99

7.2.1 Results . . . . . . . . . . . . . . . . . . . 103

7.2.2 Discussion of the Spatial Heterogeneity Results . . . . . . . . 105

8 Controlling Cardiac Arrhythmia $\quad 107$

8.1 Visualizing Shock Induced Excitation . . . . . . . . . . . . . . . . . . 109

8.2 Recruitment and Wave Propagation after the Shock . . . . . . . . . . . 111

8.3 Comparison of Shock Protocols . . . . . . . . . . . . . . . . . . . . 114

8.4 Quantification of the Excitable Gap for Far Field Shocks . . . . . . . . 116

9 Summary and Discussion $\quad 121$

9.1 Discussion and Outlook . . . . . . . . . . . . . . . . . . 123

$\begin{array}{ll}\text { References } & 125\end{array}$

$\begin{array}{ll}\text { Appendix A Pinacidil Experiments } & 137\end{array}$

A.1 Overview Plots for all Experiments . . . . . . . . . . . . . . . . . . . . . . . 138

A.2 Defibrillation Testing . . . . . . . . . . . . . . . . 144

A.3 List of all Activation Map Measurement Fits . . . . . . . . . . . . . . 146

A.4 Comparison of Cycle Length Calculations . . . . . . . . . . . . . . . 150

A.5 Alternative analysis of the Spatial Organization . . . . . . . . . . 153

$\begin{array}{lr}\text { Appendix B Code Sample } & 157\end{array}$

B.1 Windowing Function for Upstroke Detection . . . . . . . . . . . . . . . 158

Appendix C Additional Information or Details 159

C.1 Experimental Recommendations . . . . . . . . . . . . . . . . . 159

C.2 Overview over PS Identification Methods . . . . . . . . . . . . . 161 


\section{Glossary and Abbreviations}

\section{Abbreviations}

ACF Autocorrelation function

ATP Adenosine diphosphate

APD Action potential duration. $\mathrm{APD}_{X}$ at level $X$.

ATP Adenosine triphosphate. In cardiology also used for anti tachycardia pacing.

CL Cycle length, the time between two events. Typically the cell excitation but also electric shocks/pacing.

$\Delta \quad$ Triangularity index, see 4.3.4.

ECG Electrocardiogram

LV Left ventricle, the left main chamber of the heart

NPS Number of Phase Singularities

〈NPS $\rangle$ average Number of Phase Singularities within one Episode or analyzed Video.

$\mathcal{N} \quad$ Number of Phase Singularities - used in quoted/included paper.

PS Phase Singularity

RV Right ventricle, the right main chamber of the heart

VF Ventricular fibrillation - Arrhythmia associated with many spiral waves and chaotic dynamics.

VT Ventricular tachycardia - Arrhythmia associated with a fast rhythm and few stable spiral waves. 


\section{Glossary}

apex The lower tip of the heart.

charge The toplogical charge of a PS. Thus the direction, left or right, the spiral wave rotates around the PS. Can be +1 or -1 (higher orders possible theoretically).

chirality The direction of spiral wave rotation. For a PS synonymous with its charge.

core The spiral core is the area around which the spiral rotates. Used synonymous with the PS.

endocard The innermost layer of the heart, generally used to refer to dynamics close to the inside of the heart.

epicard The outer protective layer, generally used to mean close to the outside of the heart.

excitable gap The time within the activation cycle of a cardiac cell during which it can be excited by an external stimulus.

mother rotor A dominating rotor driving the fibrillation, as opposed fibrillation being driven by multiple wavelets.

pacing A periodic electrical stimulus or shock, usually using a local electrode.

Pinacidil A drug that opens ATP-sensitive potassium channels.

reentry Any spiral wave like behaviour. A reentry can be a wave travelling on a ring like structure or a free spiral wave with a PS at its core.

refractory time The time within the activation cycle of a cardiac cell during which an external stimulus cannot cause excitation.

rotor Any spiral wave like behaviour.

shock An electrical far field shock delivered through plate electrodes.

trace The temporal signal from a single camera pixel or an averaged area.

upstroke The initial phase during cardiac excitation of increasing action potential.

vulnerable window The subset of the excitable gap, during which the newly created wave will be blocked in one direction and thus may create new spirals.

wavebreak The point at which wavefront and waveback meet. Used as a synonym for a PS with a specific phase definition.

wave emitting site Any place, typically a heterogeneity, where an electrical shock excites the tissue. 



\section{Chapter 1}

\section{Introduction}

The heart is one of the most important organs in the human body. It is capable of constantly pumping blood throughout the body without any interruptions and with incredible reliability. The importance of the heart, however, means that dysfunctions can have an enormous impact. This is highlighted by the fact that heart disease is one of the largest causes of deaths in Europe [1].

A central type of cardiac dysfunctions are arrhythmias and of these especially ventricular fibrillation is immediately life-threatening. In its normal function, the heart contracts in regular intervals. During fibrillation, however, the coordinated contraction does not occur anymore and the pumping function is lost. When this happens, a strong electrical shock is typically applied to "reset" the heart - the defibrillation.

Uncoordinated fibrillatory behaviour is associated with many individual excitation waves perpetually moving through the heart $[2,3]$. The description of these waves as spirals with well-defined cores - the phase singularities - was a milestone for understanding this lethal condition. Today, phase singularities are seen as a hallmark of ventricular fibrillation and are commonly analyzed or used as a theoretical tool to study arrhythmia and defibrillation strategies.

Often the occurrence or sustaining of fibrillation can be linked to other heart diseases, that can cause changes in the heart muscle. One such example is the insufficient blood supply - ischemia -, which can change the dynamics of the arrhythmia. In many cases changes in the dynamics, such as occurring also due to restricted blood supply, are limited to some areas of the heart and create an heterogeneity. It is well known that such diseases are precursors to cardiac arrhythmia and the theoretical study of such heterogeneity has received increasing scientific attention.

Much progress has been made in understanding cardiac diseases and precursors to fibrillation including detailed simulation studies progressing towards patient specific modelling. At the same time, theoretical approaches employ phase singularity analysis as a means for characterization. Yet, the characterization of experimental data based on phase singularities has not progressed as significantly.

The understanding and improvement of defibrillation techniques is a central aim of this research. One question relates to the variations seen between fibrillation episodes 
and hearts. The success of new defibrillation approaches can vary and the reasons for these variations are elusive. One such variation can be found in the influence of ischemia. The experiments shown in this thesis were devised to quantify such variations and a main focus of this thesis is the detailed analysis of phase singularities as a means to characterizing the variations. First, the changes in phase singularity behaviour are analyzed with respect to changes in the whole organ. Second, the effect of spatial heterogeneity, which can be associated with localized ischemia, is considered. Further, variation in defibrillation success is not only related to the dynamics but also to how and where electrical shocks control the arrhythmia. A detailed characterization of the dynamics and spatial structure of phase singularities will help to better describe fibrillation. Together with insights into how shocks affect the fibrillation this can help to understand and finally improve defibrillation.

\section{Structure of the Thesis}

After introducing the main concepts concerning the heart, cardiac arrhythmia, and excitable media, a detailed introduction into the experimental methods is given. In Chapter 3 some theoretical background - mainly of the stochastic modelling approach used in Chapter 6 - is presented and existing literature reviewed. The analysis of optical mapping videos is the main topic of the thesis and the detailed analysis approach and methods are explained in Chapter 4. The results part of the thesis is structured into four chapters:

- Most of the results in the thesis are based on experiments where the drug Pinacidil was used to modify the dynamics of the heart with the idea to understand the variability in fibrillation and finally defibrillation. The result Chapter 5 details the experiments and the changes caused by Pinacidil.

- Based on the same data, Chapter 6 introduces the analysis of cardiac experimental data using a stochastic Markov model for the number of phase singularities. It discusses results with respect to Pinacidil and theoretical studies.

- The effect of heterogeneity on the dynamics is studied in Chapter 7. First, this is done from a numerical perspective. Then the chapter continues by studying experimental data with respect to such dynamical heterogeneity.

- Finally, the last result chapter discusses how electric shocks interact with fibrillation. This discussion is mainly based on exemplary studies.

At the end a summary and discussion of the results, and an outlook are given. 


\subsection{The Heart}

The heart, being the central pumping organ for the transport of blood, is vital for survival. Here, some basic properties of the heart, especially those related to cardiac arrhythmia will be presented.

Starting with its large scale structure, the mammalian heart is composed of four chambers, the left and right atria, and the left and right ventricles as shown in Figure 1.1. The overall blood flow through the body can be summarized as follows:

1. Oxygen enriched blood travels through the pulmonary veins into the left atrium.

2. During atrial contraction the blood is transported into the left ventricle.

3. As the ventricles contract blood is pressed out of the left ventricle into the aorta and through all of the body including the heart itself.

4. This blood gets de-oxygenated and reaches back to the heart into the right atrium through the superior and inferior vena cava.

5. Now, during the atria's contraction, the right atrium pumps the de-oxygenated blood into right ventricle.

6. Finally, during the ventricles' contraction, the right ventricle pushes the blood into the pulmonary artery to the lungs.

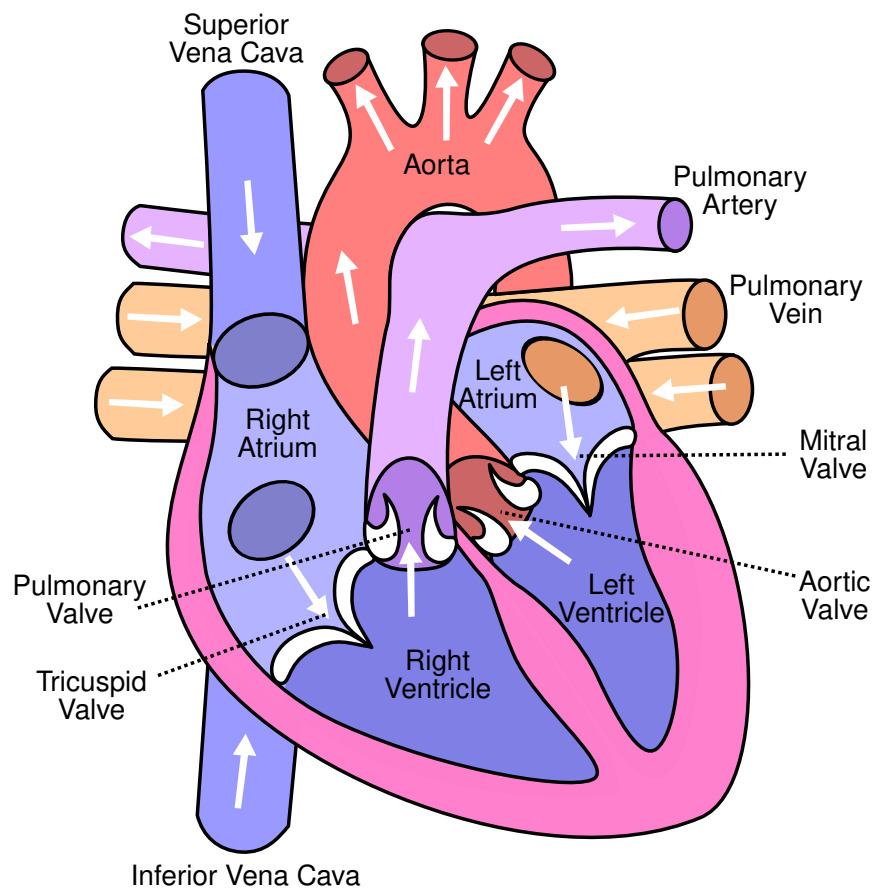

Fig. 1.1 Diagram of a human heart including the blood flow. ${ }^{1}$

\footnotetext{
${ }^{1}$ https://en.wikipedia.org/wiki/File:Diagram_of_the_human_heart_(cropped).svg
} 
Since it has to provide blood flow to the whole body, the left ventricle performs the largest part of the pumping effort. Anatomically this means that the left ventricle has thicker walls and is generally larger than the right ventricle. Also the atria, which mostly assists the ventricles, is much smaller and has thinner walls by comparison. The muscle tissue separating the left and right ventricles is called the septum and has a similar wall thickness as the rest of the left ventricle.

On smaller scales the heart is highly heterogeneous and does not have smooth walls on the inside (endocard). The so called trabeculae span the ventricles inside as ridges or bridges. Further heterogeneities are for example formed by the blood vessels which provide the heart with nutrients [4].

The cardiomyocytes are the main building blocks of the heart. They are elongated cells of about $100 \mu \mathrm{m}$ in length and $10 \mu \mathrm{m}$ in width and height, which contract along their major axis. The cells are aligned in a very regular and structured way. Sheets of cells with the same alignment wrap around the heart. At the same time their direction also changes from the inside (endocard) to the outside (epicard). This spatial organization is important for two properties of the heart. First, it explains why the wave propagation is anisotropic [5] - in the direction of cell alignment, waves travel twice as fast as in the orthogonal direction. Second, the complex cardiomyocyte alignment causes the twist like contraction observed during the heart beat. On the cellular level, more inhomogeneities are found. Clefts exist between cells, and other cell types such as fibroblasts are embedded into the tissue.

\subsubsection{Electrical Conductance}

The electrical conduction inside the heart is normally initiated by the sinoatrial node located in the right atrium. This sinoatrial node consists of pacemaker cells, which undergo periodic excitation. These pacemaker cells thus cause the normal (sinus) rhythm of the heart. After initial excitation, occurring at the sinoatrial node, waves first propagate throughout the atria causing their contraction. Between the atria and the ventricles lies the atrioventricular node. The atrioventricular node delays wave propagation of excitation waves from the atria. It then triggers the ventricular contraction through a fast conduction system and the Purkinje fibres. This causes a rapid depolarization of of the ventricles, with a wave that travels from the apex upward.

While the normal sinus rhythm as described above is associated with a fast activation of the ventricles, this is different during arrhythmia, when a much less organized wave propagation with complex wave patterns can be found. Due to the fast conduction system, in general the sinus rhythm also has a much faster propagation speed compared to activation, for example, by a local stimulus. However, cardiomyocytes do not require the fast conduction system. Instead they are directly coupled through gap junctions allowing for a wave to spread in all directions.

The wave spreading in the heart thus occurs due to an excited cell causing excitation in its neighbouring cells. In simulation studies this coupling of neighbouring cells is commonly described by a diffusion term in the membrane potential of the cells. 


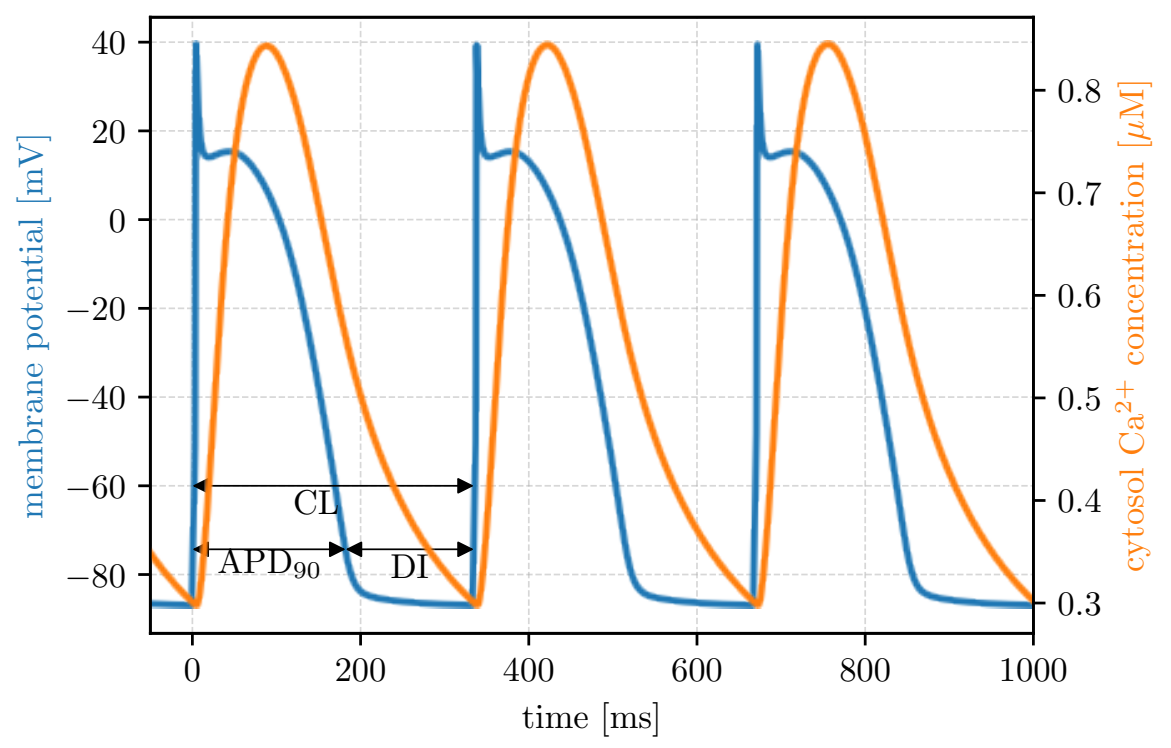

Fig. 1.2 Simulation of a rabbit ventricular myocyte paced at a frequency of $3 \mathrm{~Hz}$ and modelled using the Mahajan model [6]. The plot shows the membrane potential and cytosolic calcium concentration in the cytosol. The $\mathrm{APD}_{90}, \mathrm{DI}$, and CL indicate the action potential duration at $90 \%$ repolarization, the diastolic interval, and the basic cycle length, respectively.

The coupling enables the organized excitation and contraction of the heart. However, it also allows for the complex - arrhythmic - wave patterns which will be described later.

\subsubsection{The Cardiomyocyte and Action Potential}

The main building blocks of the heart are its muscle cells, the cardiomyocytes. Like neurons, cardiomyocytes can be triggered by an external stimulus, and then will go through a fast depolarization and subsequent repolarization as shown in in the time course of the action potential depicted in Figure 1.2.

The blue curve in Figure 1.2 shows a cardiomyocytes action potential. After a fast upstroke or increase in membrane potential, a plateau phase is reached before the cells membrane potential falls again. A commonly used characteristic is the action potential duration (APD), typically defined as the duration of the action potential at a given percentage of the maximum depolarization. Thus, $\mathrm{APD}_{90}$ is be the time spent above $10 \%$ of the resting potential and within $90 \%$ of the maximum membrane potential (compare Fig. 1.2). The time after the APD until the next upstroke is called the diastolic interval (DI) since it is associated with the relaxation, the diastole, of the heart. The time interval between two consecutive upstrokes is referred to as the cycle length (CL).

The excitation of the cell is normally caused by neighbouring cells or an outside electrical current which increases the membrane potential above a threshold and by 
that triggers the characteristic action potential described above. The heart itself is intrinsically paced periodically and can adept its frequency to external triggers like physical strain. Thus, it should be no surprise that the cardiac cells adapt to various pacing frequencies. A higher frequency of excitation will cause the action potential to shorten. Additionally, if a cell is paced too fast, it will not be able to follow the pacing anymore. This highlights two important characteristics of the cardiac system:

1. The APD restitution describes the shortening of the action potentials at higher frequencies/shorter CL. Furthermore, its APD restitution can explain some of the dynamical phenomena observed in the heart, including APD alternans: When the heart is paced at a fixed frequency the APD of consecutive beats can differ [3].

2. The refractory period, is the time during which an external stimulus is not able to excite a cell again [7,8]. A stimulus given during most of the action potential plateau will not be able to cause a new excitation.

The second property will be one of the main properties explaining how cardiac arrhythmia arises. The excitation of cells by an external stimulus, together with the refractory period, make the heart an excitable medium, which will be discussed in more detail in Section 1.2. Briefly, these properties are sufficient to explain wave patterns such as the spiral wave. In the cardiac sciences spiral waves are also often called rotors or reentry ${ }^{2}$.

A large number of ion channels are involved in the formation of cardiac action potential. One prominent ion current is the calcium release, which contributes both to the action potential and signals the contraction [9]. Another important ion is potassium. Increasing the extracellular potassium concentration $\left(\left[\mathrm{K}^{+}\right]\right)$increases the cells' resting potential. This is clinically relevant since it is the active component of the cardioplegic solution used to preserve hearts in a low energy state for transportation. Furthermore, many diseases or drugs are associated with specific ion currents and thus ionic currents are an important field in medical research.

However, the detailed ionic currents shall not be discussed here. From a dynamical system point of view, the general properties of the cell and the complexity arising through cell to cell coupling are more important. While the ionic currents are central, the properties leading to and sustaining cardiac arrhythmia are of a more fundamental nature and do not require a detailed understanding of the ionic currents in the cell.

\subsubsection{Basic Mechanisms and Modelling of the Cardiac Cell}

Cardiac cells can be modelled using electrical analogues. The single cell is characterized by its cell membrane capacitance $C_{m}$ as well as ionic currents through the cell membrane or within different parts of the cell, which cause the cell membrane potential $V_{m}$ to change [9]:

\footnotetext{
${ }^{2}$ Especially reentry may also be used to distinguish a spiral wave and wave propagation along a ring like structure.
} 


$$
\frac{\mathrm{d} V_{m}}{\mathrm{~d} t}=-\frac{\left(I_{\mathrm{Na}^{+}}+I_{\mathrm{K}^{+}}+\cdots\right)}{C_{m}}
$$

as a function of the different ion currents $I_{X}$. These are in turn regulated by ion channels in the cell membrane with their specific gating characteristics, which are often dependent on $V_{m}$ but may also be activated/deactivated by certain concentrations or even physical strain [10]. Other important components of the ionic system are active membrane enzymes (such as the $\mathrm{Na}^{+} / \mathrm{K}^{+}$-ATPase). These act as pumps which create the concentration difference between inside and outside of the cell. Through the Nernst equation this concentration difference explains the resting potential of the cardiac cell.

Models of the cardiac cell vary widely in complexity ranging from only two to dozens of variables [8]. However, as mentioned before, this thesis is focused on studying the heart from a dynamical systems point of view. So in the next sections I will describe excitable systems in more depth.

\subsection{Excitable Media}

The cardiac tissue constitutes what is called an excitable medium and as such is a part of nonlinear dynamics research. Besides the heart, other examples include Mexican waves, forest fires, or bacterial cultures.

To form a media, neighbours needs to be coupled in some form. Additionally, the basic properties of the local behaviour are the existence of:

- a quiescent or resting state, in which a cell may be excited by neighbours

- an excited stated which may also cause excitation in the surrounding medium

- a refractory state, in which a new excitation is not possible and no excitation of neighbouring cells will occur.

To give some examples, different excitable systems and their corresponding states are given in Table 1.1. At the limit of small cells, these systems can be described by partial differential equations. When describing the system as partial differential equations, at least two variables are necessary. The first variable describes the excitation dynamics while the second variable represents the refractory state and follows the first variable.

One such model is the Bär-Eiswirth model [11] which will be used later in this thesis. The local behaviour of the Bär-Eiswirth model is described by the differential 
Table 1.1 Examples of excitable systems and the corresponding states they exhibit.

\begin{tabular}{|c|c|c|c|c|}
\hline System & Resting & Excited & Refractory & Coupling \\
\hline cardiac & resting state & depolarization & $\begin{array}{l}\text { ion concentrations } \\
\text { prevent further } \\
\text { depolarization }\end{array}$ & gap junctions \\
\hline forest fire & $\begin{array}{l}\text { grown } \\
\text { vegetation }\end{array}$ & burning fire & no vegetation & flames \\
\hline $\begin{array}{l}\text { bacterial } \\
\text { growth }\end{array}$ & $\begin{array}{l}\text { plenty of } \\
\text { nutrients }\end{array}$ & $\begin{array}{l}\text { bacterial growth } \\
\text { and use of } \\
\text { nutrients }\end{array}$ & no nutrients & $\begin{array}{l}\text { expansion of } \\
\text { the colony }\end{array}$ \\
\hline $\begin{array}{l}\text { mexican } \\
\text { wave }\end{array}$ & sitting person & standing person & "tired" person & $\begin{array}{l}\text { observing } \\
\text { neighbours }\end{array}$ \\
\hline
\end{tabular}

equations:

$$
\begin{aligned}
& \frac{\mathrm{d} u}{\mathrm{~d} t}=\frac{1}{\varepsilon} \cdot u \cdot(1-u) \cdot\left(u-\frac{v+b}{a}\right) \\
& \frac{\mathrm{d} v}{\mathrm{~d} t}= \begin{cases}-v & u<\frac{1}{3} \\
1-6.75 \cdot u \cdot(u-1)^{2}-v & \frac{1}{3} \leq u \leq 1 \\
1-v & u>1\end{cases}
\end{aligned}
$$

The model parameters are $\varepsilon, a$, and $b$ with $\varepsilon=0.08, a=0.84$, and $b=0.07$.

To understand the implications of these equations it is helpful to plot the nullclines, the lines where $\mathrm{d} u / \mathrm{d} t=0$ or $\mathrm{d} v / \mathrm{d} t=0$ as shown in Figure 1.3. Additionally, the arrows indicate the derivatives given in Equation 1.2. Inspecting the figure shows that the system has a stable point when both variables have the value zero. However, when a small stimulus shifts $u$ to the green triangle indicated in the figure the self excitation causes a rise in $u$ until it plateaus when $u \lesssim 1$. Next, the slower dynamics of $v$ take over which causes $v$ to drop again and the system goes back to stable fixed point - the quiescent state. The local dynamics thus are akin an oscillator only that the oscillation requires an initial perturbation to start.

The parameter $b$ directly controls the excitation threshold and negative values would lead to oscillatory behaviour by shifting up the nullcline and making the $(0,0)$ point unstable. The parameter $a$ further modifies the position of the nullcline and thus trajectory. The excitability $\varepsilon$ is important, since it separates the time scales by ensuring that the dynamics in $u$ are generally faster than in $v$. For the upstroke to occur $u$ has to increase in value fast while $v$ is still small since otherwise an increase in $v$ would counteract the increase in $u$. Thus $\varepsilon \ll 1$ is necessary for the characteristic dynamics. 
a)

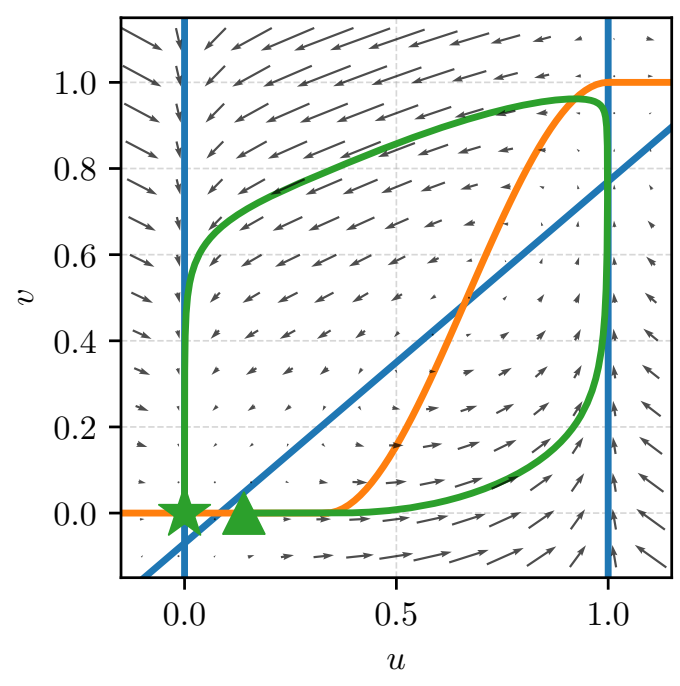

b)

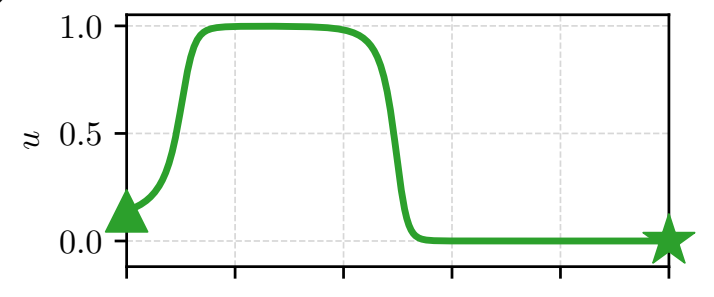

c)

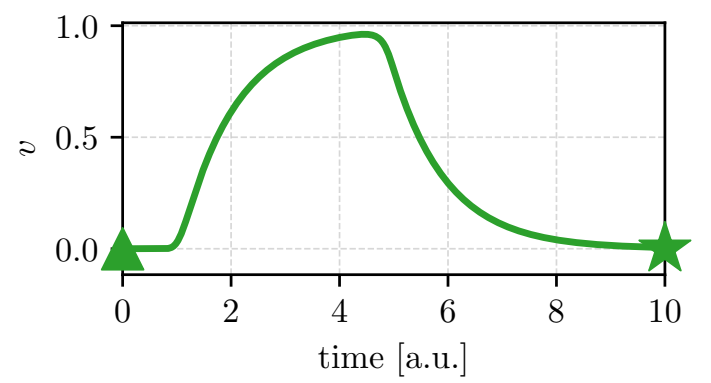

Fig. 1.3 a) Phase portrait of the Bär-Eiswirth model with parameters $\varepsilon=0.08, a=0.84$, and $b=0.07$. The blue and orange lines show the $u$ - and $v$-nullclines (zero derivatives). The arrows indicate the derivative of the system. The green line shows a trajectory starting from the triangle and ending at the stable fixed point marked by the star. $\mathbf{b}+\mathbf{c}$ ) The temporal evolution of the $u$ and $v$ variable for the line shown in (a).

\subsubsection{Local Dynamics to Spatial Organization}

To model spatial coupling in the partial differential equation of an extended medium a diffusive term is introduced into Equation 1.2 (or eq. 1.1 for general cardiac models):

$$
\frac{\partial u}{\partial t}=f(u, v)+\nabla(\mathbf{D} \nabla u) .
$$

$\mathbf{D}$ is the diffusion tensor and the equation can be simplified with $\nabla(\mathbf{D} \nabla u)=D \nabla^{2} u$ for isotropic diffusion with the corresponding diffusion constant $D$. In this model the coupling is limited to the membrane potential, although for excitable media diffusion can in general occur for all variables.

A sketch of an excitable medium in the form of a circle is shown in Figure 1.4. In (a) we see the circle fully prepared in the excitable state. A stimulus is initiated at the position indicated by the green triangle. This stimulus travels upward on both sides of the circle and finally collides at the top. On the other hand, in (b), the system is prepared with a refractory region on the left side. This means that the wave initiated from the green triangle cannot travel to the left and a wave only propagates on the right side. However, if the refractory state goes back to the resting state within the time until the wave reaches this area from the other side then the final behaviour is a perpetually counterclockwise rotating wave.

This circular representation is an example of a so called reentry in the cardiac sciences, since such circles may also occur inside the heart. Similar reentry patterns are 

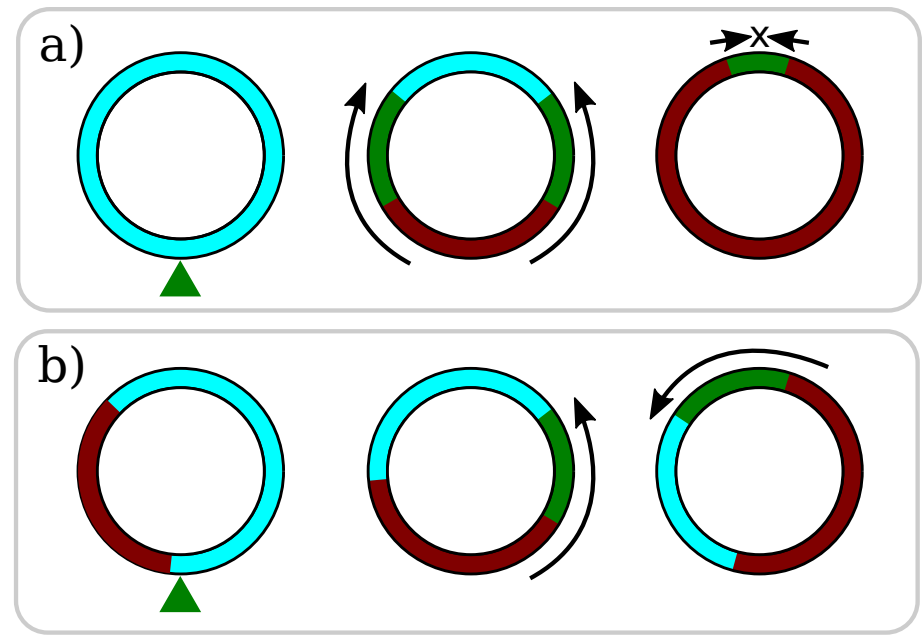

Fig. 1.4 Schematic of a circular excitable medium. Turquoise indicates the resting, green excited, and dark red the refractory state. a) A stimulus is given at the green triangle position into a fully resting system. b) A stimulus is given when the system is prepared with a refractory area on the left side. (Further explanation in the text.)
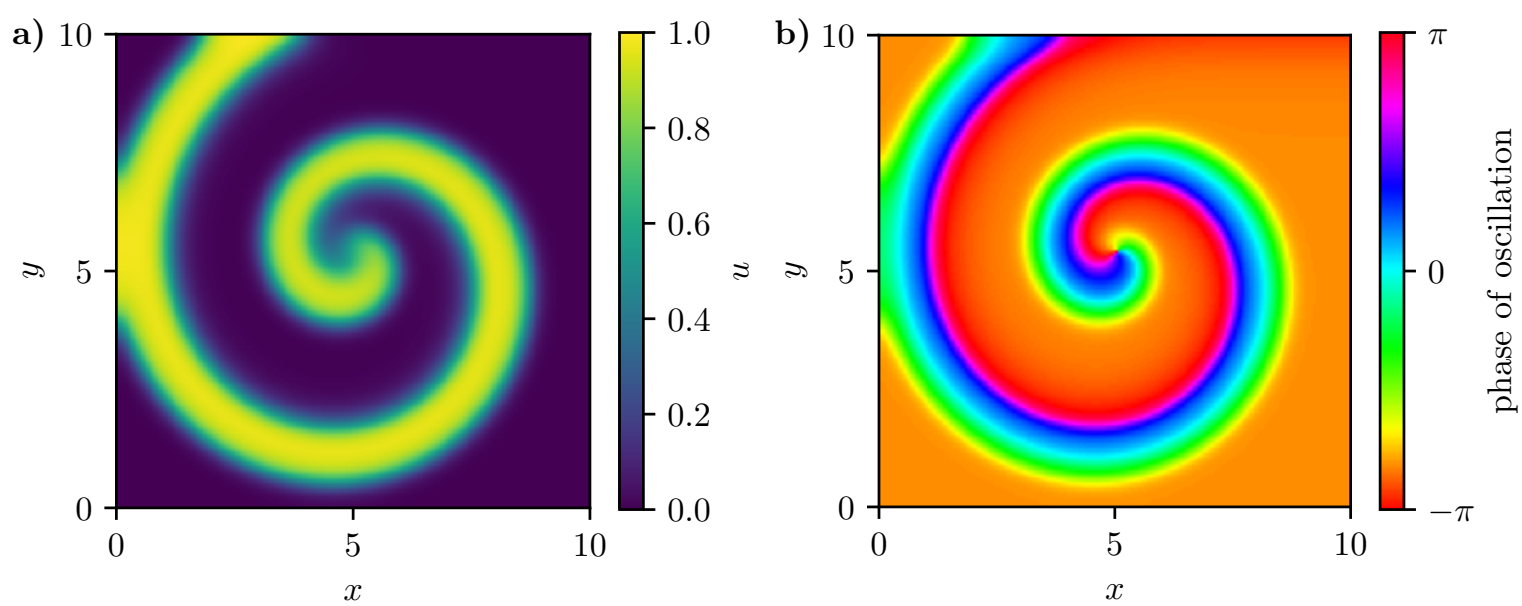

Fig. 1.5 Example of a spiral wave activation in the two-dimenional Bär-Eiswirth model (eq. 1.2) with $a=0.84, b=0.07, \varepsilon=0.07$, and $D=0.1$ (see eq. 1.3). a) The $u$ variable, which corresponds roughly to the cardiac membrane potential and $\mathbf{b}$ ) the phase of the oscillation which is defined as the angle around the point at $u=0.65$ and $v=0.45$ in Figure 1.3. In this phase image, the spiral has a well defined core where all phases meet the phase singularity (PS). 
also possible when extending the ring to a two dimensional domain. When extending the ring to become a circle, one finds that there is a point where all states (excitable, excited, and refractory) must meet. This point is the spiral core and will later be associated with a phase singularity. When extending the circle to the outside, the waves will bend to form a spiral.

Such a spiral wave as modelled in the Bär-Eiswirth model is shown in Figure 1.5 (a). Further Panel (b) shows the phase associated with the local state. This is defined by:

$$
\varphi(x, y)=\operatorname{atan} 2(u(x, y)-0.65, v(x, y)-0.45)
$$

It indicates the local state with respect to its oscillation like behaviour.

\section{Phase Singularities}

The spiral is clearly visible in both panels of Figure 1.5, but the core of the spiral is a distinct point for the phase. Directly at the core all phase values meet and the image is not differentiable. This singular point is called a phase singularity (PS) and identifies spiral wave activity. A line integral around the PS will integrate to $2 \pi$ or $-2 \pi$ depending the direction in which the spiral rotates.

PS are topological defects and have conservation properties. When the phase changes continuously with time the PS can move through the domain. However, PS can only be created in pairs of opposing chiraltity or at a boundary. Further, a PS can only be removed either when colliding and thus annihilating with one of opposing chirality, or when colliding with a boundary.

This thesis only analyzes two dimensional data, however, the heart is a three dimensional tissue. In general the PS will be associated with a so called filament in three dimensions and the spiral wave becomes a scroll wave (compare Fig. 1.6). The PS is then the point where such a filament ends at the boundary.

\subsection{Cardiac Arrhythmia}

After reviewing the basic mechanisms of the dynamics of the healthy heart, as well as the properties of excitable media, cardiac arrhythmias can now be understood in these terms. On the one hand the heart can be thought of as a self-sustained oscillator causing regular pumping, where oscillation is driven by localized pacemakers. On the other hand, the entire heart tissue is an excitable medium and thus can exhibit selfsustained spiral wave activation patterns without the need for pacemakers. Such spiral wave activation patterns form the cardiac arrhythmia: atrial flutter (AFl) and atrial fibrillation (AFib) as well as ventricular tachicardia (VT) and ventricular fibrillation (VF). VF will be the main focus in this thesis. VT is much faster than the normal sinus rhythm and is often associated with one or few spiral wave patterns on the heart 
a)

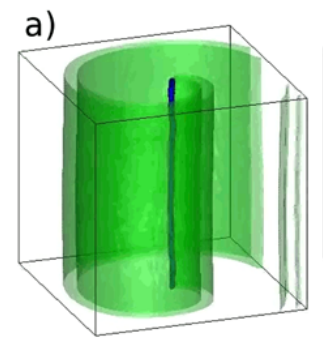

e)

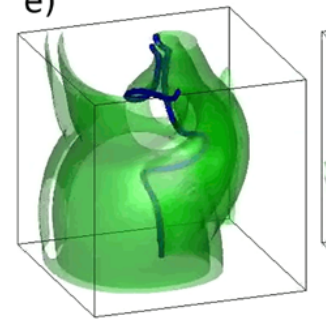

b)

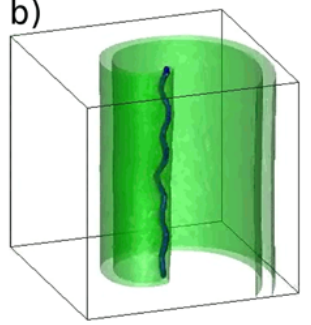

f)

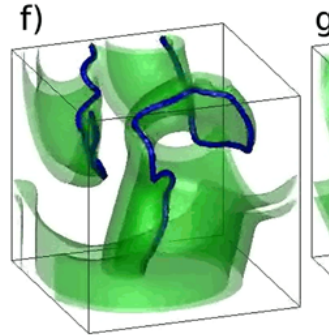

c)

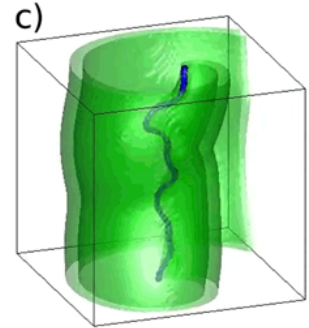

g)

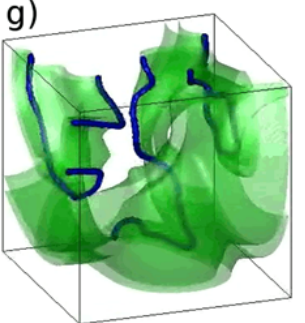

d)

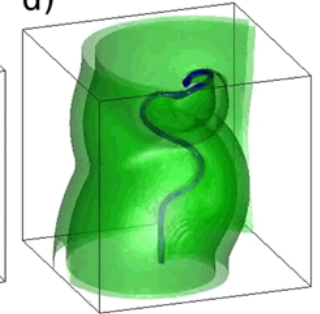

h)

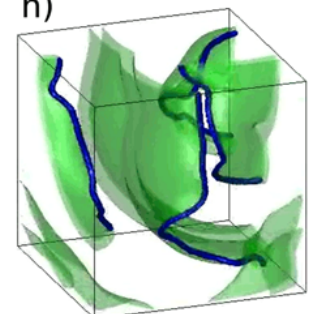

Fig. 1.6 Spiral wave breakup due to negative filament tension, reproduced from [12]. The initially straight filament at the centre of the scroll wave (three dimensional spiral wave) elongates from Panels (a) to (d). In (e) two filaments exist which create three PS on the top surface. Panels (f) to (h) show the further evolution. The model system is a Luo-Rudy model with modified parameters: The values of $G_{\mathrm{Na}}$ and $G_{\mathrm{si}}$ are reduced to lower tissue excitability. Reprinted by permission from Springer Nature Customer Service Center GmbH: Springer Bulletin of Mathematical Biology "Negative Tension of Scroll Wave Filaments and Turbulence in Three-Dimensional Excitable Media and Application in Cardiac Dynamics" Sergio Alonso, Markus Bär, and Alexander V. Panfilov. (O) Society for Mathematical Biology 2012 .

which follow periodic dynamics. VF is characterized by a higher number of spiral waves, an even faster rhythm, and fully complex dynamics on the ventricles.

Such activity associated with VF implies that the activation of the heart is not coherent anymore, and blood cannot be pumped. Thus, especially VF is immediately life threatening and requires intervention. This is achieved by electrical defibrillation where high energy electrical shocks are applied to the heart (compare Sec. 1.5).

\subsection{Spiral Wave Breakup}

Phase singularities (PS) or their three dimensional counterparts, phase filaments, describe the existing spiral wave patterns in the heart. During ventricular fibrillation new PS are both continuously created and annihilated in pairs or collide with the boundary. During the process of PS creation, existing spiral waves break up into new ones. This can occur due to various mechanisms of spiral wave breakup observed in the heart and models of cardiac tissue $[13,14]$. Such breakup can occur at, or be promoted by, heterogeneities such as shown in the porcine heart in [15]. For example wave breakup occurs when a wave catches up with a previous one and partially collides with or slows due to the previous wave. Such a wave may split up into multiple new 
waves and thus create new PS pairs.

Many mechanisms of spiral wave breakup are listed in detail by Fenton et al. in [14]. Most of these mechanisms exist both in two as well as three dimensions. There is, however, a notable exception. The creation of new PS due to negative filament tension occurs only in three dimensions. Figure 1.6 from [12] shows such a breakup due to the elongation of filaments and the creation of new PS in the Luo-Rudy model [16].

\subsection{Effect of External Electric Fields}

Although the mechanisms by which defibrillation stops the arrhythmia are complex, the basic principles of how an electric stimulus affects the cardiac tissue are well known. A local current injected into the cell will cause a change in the membrane potential of the cell and thus cause the excitation through opening of ion channels. The effect of an electric field or current applied over a tissue can be understood in the bidomain model. In the bidomain model the intracellular and extracellular domains are described separately. While the intracellular domain describes currents flowing through the cells and gap junctions between them, the extracellular domain has different electrical properties and describes currents flowing outside the cells. The two domains are coupled through the cell membrane so that electrical currents can flow both in the intra- and extracellular domain as well as between the two. The electric current will split up according to the resistivity within and around the cells to flow either through or around them (intra- or extracellular). Due to different resistances in the intra- vs. extracellular domains, different currents flow through them and a net voltage difference is created when the currents enter or exit the intracellular domain. This occurs when the resistances of either domain changes locally or the tissue ends.

This theory suggests that the effect of electrical shocks depends on geometry, encounters with tissue boundary, blood vessels, changes in tissue properties, changes in anisotropy ratios of the resistances, and even small scale heterogeneities such as clefts between cells. The general effects are readily observed in experiments, and both theoretical and numerical studies have predicted more in-depth properties [17-20].

The locations excited by the shock are also called virtual electrodes, wave emitting sites, or wave emitting heterogeneities. Whether or not a specific location is recruited - enough current flows - depends on the shock strength. However, it also depends for example on the size of the heterogeneity (compare Sec. 3.1) and the relation of the required shock strength for different sizes is an important part of research into improved techniques for defibrillation $[4,21]$.

\section{Excitable Gap}

During their activation cycle cardiac cells go through a refractory phase, where they cannot be excited by an external stimulus, be it from neighbouring cells or an electrical shock. In general, the time span for which the cell cannot be excited again is dependent on the strength of the electrical shock. After the refractory time the cell can be excited 
again until activation occurred. This time span is referred to as excitable gap. An early verification of its existence for local pacing in the heart was for example done in [22, 23]. An overview on how cells or spiral waves on the heart will respond to electrical shocks can be found in [24].

\subsection{Ischemia}

Ischemia describes the state of the cardiac tissue in the case of insufficient perfusion by blood. This state is associated with multiple changes on the cellular level as reviewed in great detail by Carmeliet in [25]. A second effect of insufficient perfusion is insufficient waste disposal. All of this causes multiple changes in concentrations on the cellular level. Three of the most important ones are: First, an increase in the extracellular potassium concentration. Second, an increase of the ADP concentration while the ATP concentration decreases. Third the lack of oxygen causes a switch to the anaerobic metabolism, which additionally causes acidosis.

All of these changes directly affect the action potential shape and the properties of an arrhythmia. One of the most prominent changes is the shortening of the action potential which is largely due to the ATP sensitive potassium channels. This finally also leads to a more triangular shape as for example observed for the rabbit heart in [26]. For this triangular shape, compare also Section 4.3.4. These changes further cause a general decrease in the excitability largely due to the increased extracellular potassium concentration [25].

In-vivo a VF will also lead to ischemia. Because of this ischemia the arrhythmia is not stable and changes over time. Typically the arrhythmic frequency initially increases but then decreases again [27-29]. At the same time the conduction velocity also decreases after long periods of ischemia [28, 30]. Commonly, an increase of the heterogeneity in frequency or action potential duration is also observed [27, 31]. 


\section{Chapter 2}

\section{Experimental Methods}

In cardiac sciences the Langendorff perfusion is the fundamental method of studying the heart as a whole organ in an ex-vivo setting [32]. The Langendorff perfusion allows to keep an extracted heart under physiological conditions for several hours and thus is an ideal method for the studies presented in this thesis.

The setup is shown in Figure 2.1 and is described in more details in the following sections. Some more general recommendations, mostly concerning defibrillation trials, can be found in the Appendix C.1 (p. 159).

The main reason for the use of perfused hearts is that it enables a more detailed study for example by using optical techniques. In the late 1980s optical mapping measurements of cardiac cells and whole hearts became possible and spurted a multitude of studies such as [34-36] and later [37-39]. The technique of optical mapping will be explained in more detail in Section 2.2. It allows optical high speed and high resolution measurements for example of the membrane potential or the intracellular calcium concentration. Both are central to the cardiomyocytes activation cycle. While previously recordings could be done using electrical signals, for the first time it was thus possible to image cardiac arrhythmia at a high spatial resolution. For example, this allowed to verify the theory that cardiac arrhythmias are associated with spiral waves and phase singularities.

\subsection{Langendorff Perfusion}

The Langendorff perfused heart is retrogradely perfused through the aorta using either blood or a physiological buffer solution. For a short distance, the flow is thus opposite to the normal pumping of the heart. Comparing with Figure 1.1 the blood vessels providing nutrients to the heart start from the aorta, which is the main artery. Perfusion of the heart thus occurs the same way as normally, while the left ventricle would pump against an externally applied pressure.

In all experiments shown here a buffering solution is used to provide the excised heart with the nutrients and keep it in physiological conditions. The most important requirements for the perfusion are: 


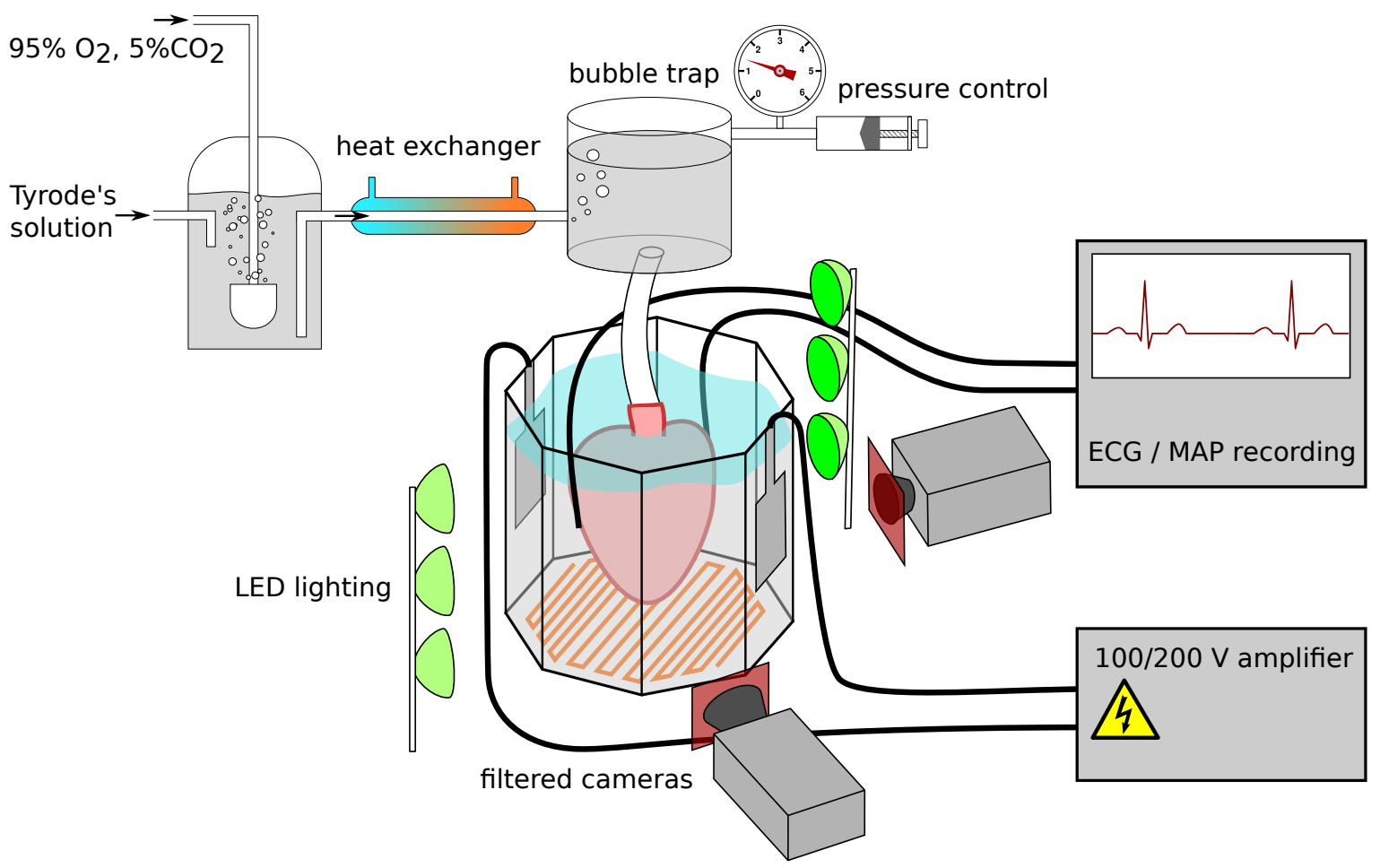

Fig. 2.1 Sketch of the experimental setup used for the whole heart experiments. The heart is perfused and submersed in a bath of Tyrode's solution keeping it in physiological conditions. Additionally, optical and electrical measurements can be taken and electrical pacing or shocks can be applied. Sketch modified from the original in [33] created by Daniel Hornung.

- Correct pH value of about 7.4

- A physiological temperature of $37^{\circ} \mathrm{C}^{1}$.

- Physiological ion concentrations

- Glucose and oxygen

- Correct pressure and/or flow through the blood vessels.

In the experiments shown here this is achieved using Tyrode's solution, a bicarbonate and phosphor buffering solution. The exact composition varies slightly among different experiments shown in the thesis, however the basic and most common composition is given in Table 2.1 .

Bubbling the solution with carbogen (95\% oxygen, $5 \%$ carbondioxide) provides oxygen and is necessary for the buffer solution to achieve a stable $\mathrm{pH}$.

The general circulation is shown in Figure 2.1. First, the solution is preheated and oxygenated in a reservoir at physiological temperature. Then it is pumped into a bubble trap which ensures that no air bubbles can reach the heart, since these

\footnotetext{
${ }^{1}$ This is popular although the typical body temperature of rabbits is somewhat higher than that of humans as pointed out by our veterinarians [40].
} 
Table 2.1 Composition of the Tyrode's solution used for rabbit experiments for 15 liters of solution.

\begin{tabular}{lcc}
\hline Component & Concentration $[\mathrm{mM}]$ & Weight $(15 \mathrm{l})[\mathrm{g}]$ \\
\hline $\mathrm{NaCl}$ & 125 & 109.575 \\
$\mathrm{KCl}$ & 4.5 & 5.025 \\
$\mathrm{MgCl}_{2}\left(6 \mathrm{H}_{2} \mathrm{O}\right)$ & 0.5 & 1.524 \\
$\mathrm{CaCl}_{2}$ & 1.8 & 2.996 \\
$\mathrm{NaH}_{2} \mathrm{PO}_{4}\left(\mathrm{H}_{2} \mathrm{O}\right)$ & 1.8 & 3.72 \\
$\mathrm{NaHCO}_{3}$ & 24 & 30.15 \\
Glucose & 5.5 & 14.85 \\
\hline
\end{tabular}

could block blood vessels. Here the pressure can be adjusted and is electronically measured. The heart is attached directly below the bubble trap hanging inside a bath with controlled heating to maintain physiological temperature.

\section{Preparation of the Heart}

All experiments shown in the thesis were performed with hearts from New Zealand white rabbits. The rabbits were injected with Heparin (2000 I.E.) and then euthanized using an overdose of Thiopental. Before excision, rabbits were further injected with cardioplegic solution. Then the hearts were removed and immediately submerged into ice-cold cardioplegic solution and finally connected to the perfusion setup. ${ }^{2}$

\subsubsection{Contraction Uncoupling}

The heart intrinsically moves strongly. But to achieve reliable data in the optical mapping, all movements have to be avoided ${ }^{3}$. In some optical mapping studies this is achieved by mechanical constraint [28, 43], however, in most studies chemical agents inhibiting the myosin, the active structure in muscle cell contraction, are employed. Blebbistatin is currently most commonly used for contraction uncoupling in whole heart experiments. The effect of Blebbistatin on the electrophysiology of the heart is thought to be small $[44,45]$. Blebbistatin is unstable when illuminated with blue light $(450-490 \mathrm{~nm})$. In principle, Blebbistatin as well as the products of its photo-instability are toxic to cells. Another, downside of Blebbistatin is its low solubility and possible crystallization [45]. Very recently, improved Blebbistatin derivatives were developed which solve some of these issues [46, 47]. Especially para-Aminoblebbistatin may be a promising enhancement in the future [47].

However, for the purpose of this study, contraction uncoupling is necessary to achieve sufficiently good data for analysis. Blebbistatin is a very reliable tool which is thought to have little effect besides the intended suppression of all movement. Thus,

\footnotetext{
${ }^{2}$ The experiment on the 2018-08-22 used a different drug for euthanasia.

${ }^{3}$ Or reliable tracking needs to be done in post processing, which is active research [41, 42].
} 


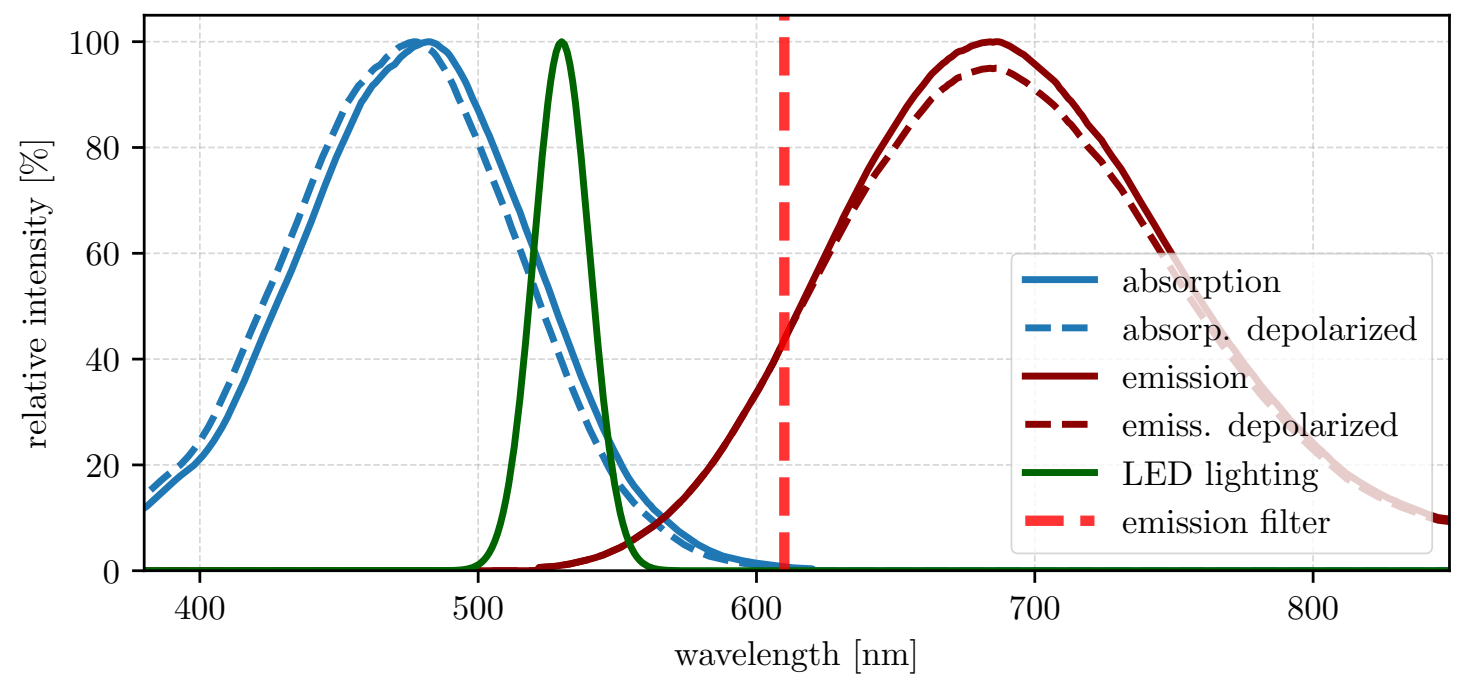

Fig. 2.2 Sketch of the optical properties of di-4-ANEPPS and the corresponding filters and lighting. The blue and red curves show the absorption and emission spectra of di-4-ANEPPS (data from Thermo Fischer Scientific) and a sketch both of the shift due to depolarization in the absorption as well as the emission spectra. Both the excitation filter and LED lighting are indicated. Compare [41] for quantitative values.

in the experiments shown in this thesis, a concentration between $5 \mu \mathrm{M}$ and $10 \mu \mathrm{M}$ was used.

\subsection{Optical Mapping}

Optical mapping techniques of membrane potential used in cardiac sciences were made possible by the development of dyes by Leslie Loew and co-workers in the 1980s [48-50] and became a fundamental method for research. These techniques enable the researcher to optically record membrane potential changes or, using other dyes, intracellular calcium changes on the surface of the heart. One requirement for the fluorescent dye in cardiac research is that it must respond to changes in action potential with microsecond resolution. This reaction time is especially short for many voltage sensitive dyes, causing no limitation to the temporal resolution.

The data analyzed in this thesis is based on the voltage sensitive dye di-4-ANEPPS. This dye attaches to the cell membrane. It then slightly shifts its absorption and emission spectra as a function of the membrane potential. Using green or blue light to excite the dye will emit light in the red range. The main effect is that with increasing membrane potential, the absorption spectrum shifts towards shorter wavelengths. Both spectra and their shift when the cell is depolarized are sketched in Figure 2.2.

To image the change due to depolarization, the brightness of the signal needs to change. This means that little or no signal is found if excitation is done around 
the peak of the fluorescent absorption. If the dye was excited around the peak, the intensity at this frequency would be hardly affected by the shift of the spectrum. On the other hand, the best signals are achieved with lighting chosen on the right flank in the cyan or green range. Using this light, the absorption efficacy decreases during the action potential and thus a decrease in fluorescent intensity $F$ is recorded - apparent by a larger difference between the blue and the dashed blue line. In theory a fractional change $\Delta F / F$ of about $9 \%$ for a $100 \mathrm{mV}$ change in membrane potential is possible [49]. Note that the deflection recorded for voltage data is thus typically negative in the raw intensity data. In practice, with the bath, non-active tissue and non-perfect staining, a fractional change of about $4.5 \%$ can be expected during sinus rhythm, although at the beginning of an experiment it can be well above $7 \%$.

\section{Optical Setup}

The setup uses high speed lenses with a maximum aperature of $0.95 / f$ and a focal lengths of $25 \mathrm{~mm}$ (Navitar), using spacers as appropriate to adjust the working distance. To record the video data we employ high speed EMCCD ${ }^{4}$ Cameras (Photometrics, Evolve or Cascade) recording at $500 \mathrm{~Hz}$ and $128 \times 128$ pixels. While having a relatively low spatial resolution, this resolution is sufficient for the data analysis and the cameras have very low readout noise. While not fully resolving the upstroke, the temporal resolution of $2 \mathrm{~ms}$ is sufficient fast to capture wave propagation on the heart. In our lab four of these cameras are mounted around the heart and recorded simultaneously as shown later in the Figure 4.1 (a).

Lighting is provided using 10 Tri-Star (each three) mounted LUXEON Rebel green (530 $\pm 10 \mathrm{~nm}$ half-width) LEDs. ${ }^{5}$ These are additionally highpass filtered at a cutoff wavelength of $560 \mathrm{~nm}$. The $530 \mathrm{~nm}$ is chosen to lie around the wavelengths where the highest change due to the absorption frequency shift is expected.

\subsubsection{Limitations of Optical Mapping}

The optical mapping resolution is limited by the sensors spatial resolution and readout speed. However, the largest limitation to the optical quality is the limited change in fluorescent intensity that occurs during excitation. Optimizing this fraction fluorescent change is a way to reduce most other artifacts as well. However, the fractional fluorescence is largely a property of the dye. The following lists different reasons limiting the video quality or precision:

- The exposure time used throughout this study is $2 \mathrm{~ms}$.

- The resolution of $128 \times 128$ pixels translates into a spatial resolution of about $0.2 \mathrm{~mm}$. This is reduced by spatial smoothing during post processing.

\footnotetext{
${ }^{4}$ EMCCD stands for electron multiplying charge-coupled device. These are camera sensors similar to typical CCD sensors, but implement an additional amplification for use in low light applications.

${ }^{5}$ Some of the recordings use a slightly different LED setup.
} 
- The Poisson noise due to the limited number of photons detected by the CCD is the largest stochastic image noise. This noise is important since the the changes in the fluorescence strength during excitation are small compared to the total signal strength.

- Even small motion can severely limit the quality. This is because the staining is not homogeneous and the difference in fluorescent activity of close pixels can be large compared to the intensity change due to the action potential. This means that even subpixel movements can cause artifacts.

- Numerical studies suggest that photon scattering and the lights penetration depth may be a larger factor limiting the spatial resolution than the CCD chip resolution itself $[51,52]$.

- Photobleaching, washout and internalization [53] of the dye reduces the quality over time ${ }^{6}$, though it may be partially offset by restaining during the experiment.

- In some cases bubbles in the bath can impair the image quality and cause fluctuating signals. These are for example created by defibrillation shocks.

A good overview of many aspects of optical mapping can be found for example in [54].

\section{$2.3 \quad$ Further Measurements}

Additionally to optical measurements, one or more ECGs are recorded for all experiments using electrodes placed around the heart. Both single and multiple electrodes setups where developed in house by Laura Diaz-Rodriguez to provide high quality and shock resistant amplification with a short blind time after the shocks.

Further, the pressure was continuously recorded and both $\mathrm{pH}$ and flow rate are monitored regularly during the experiments.

\subsection{Far Field Shocks Protocols and Local Pacing}

In all experiments far field shocks were applied using a high performance amplifier (BOP 100-10MG, Kepco Power Supply, USA). The shock electrodes are $3.5 \mathrm{~cm}$ times $5 \mathrm{~cm}$ stainless steel plates at a distance of $9.5 \mathrm{~cm}$, in general the impedance of the bath with the solution and the heart is about $20 \Omega$. These plate electrodes are used both for defibrillation and all other far field shocks. Local pacing was performed with tungsten tipped micro electrodes (FHC, USA) using a current source (STM100C, BIOPAC Systems, USA). The local electrode is used for induction of the arrhythmia or to pace the heart at a different frequency than the sinus frequency.

\footnotetext{
${ }^{6}$ It should be noted that Ref. [53] suggests half life times of both $F$ and $\Delta F / F$ which seem much smaller than experienced in our setup with typically relatively stable image quality over several hours.
} 


\subsection{Software and Experimental Control}

In the lab continuous recording of all time series, including for example ECG and pressure, is performed using an MP 150 device (BIOPAC Systems). The control of data acquisition and is handled by a custom Python software (pulsar) written by my colleagues and myself. This program controls a National Instruments NI USB-6259 data acquisition unit. This provides or controls:

- the output signal for local pacing

- the output signal for far field shocks

- all necessary electrical switches

- triggers for synchronized recording of the cameras

- remote control of the camera software for convenience

- recording of the ECGs synchronized to the cameras

- Powerful logging to simplify data analysis and notes taking

For video recording, a custom made software $(\mathrm{C}++)$ developed primarily by my colleague Johannes Schröder-Schetelig and Christoph Nikic is used.

The setup allows to perform live analysis of multiple ECGs and camera traces and it can deliver specific pacing protocols based on these signals. This includes calculation of the shock period for various shock protocols but also timing a shock based on the analysis of either ECG signals or camera traces. An important advantage of using a central control device is that the video data, ECG input and all electrical shocks are recorded at the same time without any, or with a known fixed offset. This greatly simplifies analysis.

\subsection{Induction and Termination of VF}

The main focus of the research in the experiments shown here is to study cardiac arrhythmia. For this, first initiation of the ventricular fibrillation is necessary. Then the arrhythmia and finally the defibrillation can be studied.

\subsubsection{Induction Protocol}

Induction of ventricular fibrillation was done using the local pacing electrode placed typically on the left ventricle. A biphasic rectangular stimulus with $3 \mathrm{~ms}$ total pulse duration and inverted polarity after $1.5 \mathrm{~ms}$ was used. Induction was performed well above the minimum required strength necessary for pacing the heart at a slow frequency (such as with a $300 \mathrm{~ms}$ period) and usually at $50 \mathrm{~Hz}$ for a duration of $3 \mathrm{~s}$ to $10 \mathrm{~s}$. In some cases, repeated or longer inductions were necessary or a lower frequency in the range of $20 \mathrm{~Hz}$ to $50 \mathrm{~Hz}$ was used in order to induce fibrillation. 


\subsubsection{Defibrillation}

In general defibrillation was achieved using $7 \mathrm{~ms}$ biphasic rectangular shocks with an inverted polarity after $5 \mathrm{~ms}(5 \mathrm{~ms}+2 \mathrm{~ms})$. The voltage for both shock parts is identical and adjusted for each defibrillation attempt. Three different strategies were employed in the experiments shown in this thesis:

- 1 Shock: A single, bi-phasic defibrillation shock.

- 5 Shocks: Five identical shocks given at a period that is a factor of 1.2 slower than the frequency of the arrhythmia as determined from the ECG.

- 50 Shocks: 50 shocks using overdrive pacing about 10\% faster than the frequency of the arrhythmia.

After any successful defibrillation, the heart was allowed to recover for a minimum of about five minutes.

Various defibrillation techniques have been studied intensively in many contexts. For example overdrive [4, 55-58] and underdrive [33, 59-61] pacing as well as shock forms [62]. All of the experiments done here were to some degree designed to compare such methods. However, most of the analysis presented in this thesis will be independent of that. 


\section{Chapter 3}

\section{Theoretical Background}

In this chapter, the theoretical background with respect to the analysis performed in the result chapters is given. Since the main focus of the thesis is the analysis of experimental data the chapter is limited to two parts:

1. A brief review of the theory for activation time analysis with the expectation of a power law, which is used in Chapter 5.

2. An introduction and overview over the literature of defect mediated turbulence. This introduces the stochastic Markov model used in Chapter 6.

\subsection{Activation Maps and Time}

In this section, I will briefly review the theory of activation times - i.e. the time after a shock until the whole tissue has been excited - as laid out in [4]. This theory motivates the calculation of power law exponents shown in Chapter 5.

\subsubsection{Theoretical Foundation of Wave Emission at Boundaries}

While a full understanding of boundary effects cannot be derived analytically and is only approachable using numerical methods using the bidomain model, it is possible to derive approximate solutions for simple boundary conditions such as circular non-conducting heterogeneities in isotropic media [4, 18, 20,63]. These theoretical considerations rely on a few assumptions and approximations:

1. Isotropic, homogeneous medium

2. Monodomain approximation of the cardiac bidomain

3. Low field strength approximation.

However, the qualitative results of wave emission site recruitment and general intuition can be confirmed in complex simulations [19] and in cell culture experiments [18]. 


\subsubsection{From Circular Boundaries to Activation Times}

Assuming a certain field strength, there is a minimum size at which non conducting circular enclosures will be activated. As laid out by Luther and Fenton et al. in [4] these considerations may be applied to the vasculature. If one assumes a homogeneous distribution of vasculature in the tissue and a constant propagation velocity, it is possible to make a prediction for the time after a shock that is required to activate the whole volume or surface of a three or two dimensional tissue.

To approximate this activation time it is necessary to know the size distribution of the wave emitting sites. These are assumed to follow a size probability distribution for their radius given by:

$$
\mathrm{P}(r) \propto r^{\alpha}
$$

with the constant $\alpha<-1$. This formula can describe the vasculature for sufficiently small blood vessel sizes as shown in references [4,33] and references therein.

Then the density of wave emitting sites with a radius or $r_{\min }$ or larger is given by its number $N\left(r>r_{\min }\right)$ and the volume $\mathrm{V}$ :

$$
\begin{aligned}
\rho(r) & =\frac{N\left(r>r_{\min }\right)}{V}=\int_{r_{\min }}^{\infty} \mathrm{P}(r) \mathrm{d} r \\
& \propto \int_{r_{\min }}^{\infty} r^{\alpha}=\left[\frac{1}{\alpha+1} r^{\alpha+1}\right]_{r_{\min }}^{\infty}
\end{aligned}
$$

and with the assumption of $\alpha<-1$ :

$$
\rho(r) \propto 0-\frac{1}{\alpha+1} r_{\min }^{\alpha+1}
$$

substituting the approximation $r_{\min } \propto \frac{1}{E}$ which was derived in $[4,63]$ gives:

$$
\rho(E) \propto E^{-(\alpha-1)} .
$$

Equation 3.5 thus gives an estimation of the density of new waves created by a shock of electric field strength $E$. These waves will travel in all directions from the emission site. For a given density $\rho$, the distance that has to be covered by a single site can be estimated by:

$$
d(\rho) \propto \rho^{\frac{1}{D}}
$$



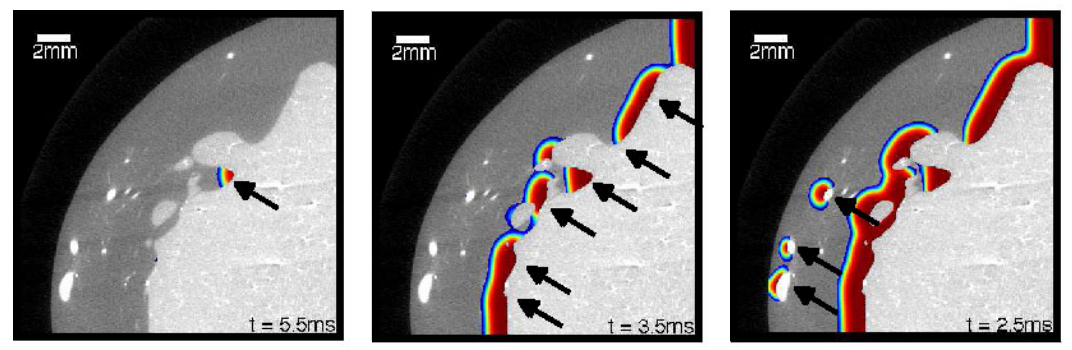

Fig. 3.1 Numerical model of activation on a rabbit LV geometry (micro-CT scan) with increasing field strengths from left to right $(0.2 \mathrm{~V} / \mathrm{cm}, 0.4 \mathrm{~V} / \mathrm{cm}$, and $1.0 \mathrm{~V} / \mathrm{cm})$. The colours indicate the membrane potential from $-80 \mathrm{mV}$ to $+20 \mathrm{mV}$ (blue to red). Figure modified from [18].

with the dimension $D$. Thus, the time $\tau$ necessary to cover this distance, based on the wave propagation speed $v$ is:

$$
\tau(\rho) \propto \frac{\rho^{\frac{1}{D}}}{v}
$$

Substituting Equation (3.5) gives:

$$
\tau(E) \propto E^{\frac{-(\alpha-1)}{D}}=E^{-\beta}
$$

with $\beta=(\alpha-1) / D$.

This gives a theoretical foundation for the expectation that, for sufficiently high field strengths, a power law for the activation time $\tau$ with respect to the field strength $E$ is expected. This relationship is based on the main assumptions that the underlying heterogeneities are circular and follow a size distribution well described by a power law.

\subsubsection{Typically Activated Areas}

As mentioned in the previous section more sites get activated by an external shock when the shock voltage is increased. To give a better intuition, Figure 3.1 shows an example from a simulation with increasing field strengths. In this study the first site activated is a protuberance (left panel). Then the endocardial wall is activated in many areas and finally the larger blood vessels are activated. More detailed simulations for the rabbit heart can be found in [19]. 


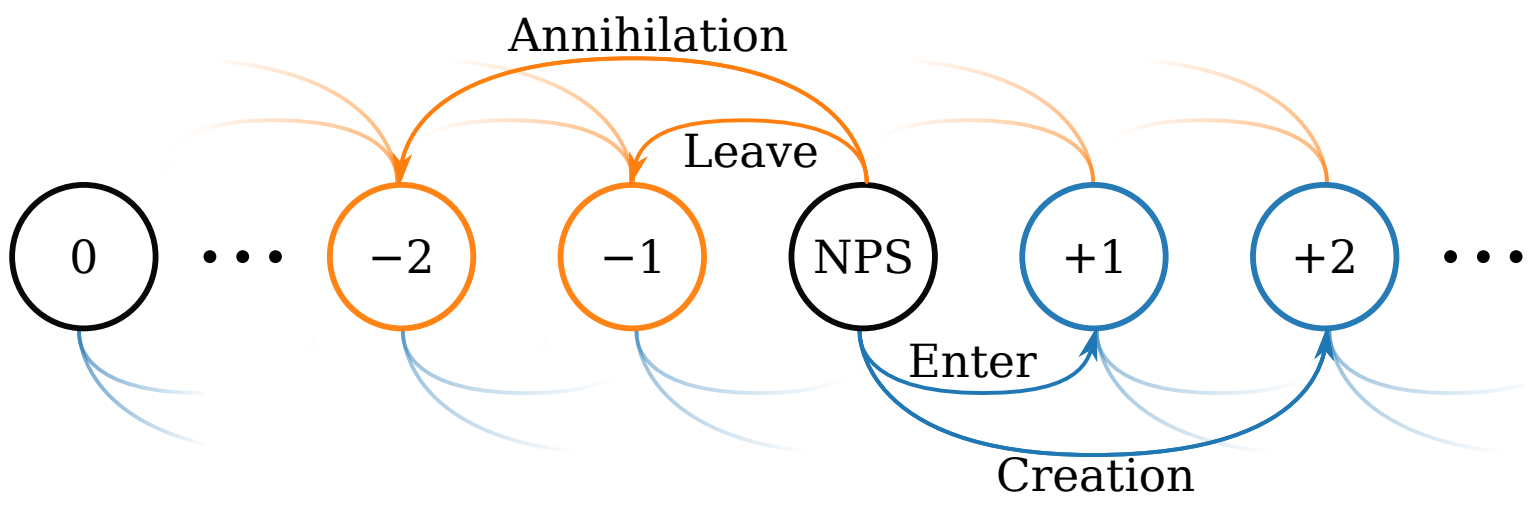

Fig. 3.2 Illustration of the Markov process. For a given number of PS (NPS) the four possible transitions are shown. The only exception is for zero and one NPS where further removal of PS is either not possible at all or not possible through pair annihilation.

\subsection{Defect Mediated Turbulence and Markov Chain Modelling}

The properties of the cardiac dynamics during arrhythmia can be likened to those of other pattern forming systems such as hydrodynamic, turbulent ones. Also in turbulent systems topological defects occur which are comparable to the phase singularities (PS) observed in cardiac dynamics. Individual PS may behave similar to gas particles moving colliding and, unlike gas particles, randomly appearing. Viewing their paths and kinematics as, to a certain degree, random suggest the possibility of modelling the behaviour of the current number of PS (NPS) over time as a stochastic process.

For many turbulent systems the stochastic properties of the defect dynamics have been analyzed previously both experimentally and numerically [64-74]. The focus in these studies is to describe the stochastic properties of the number of defects and, using numerical models, to form a deeper understanding of the system properties.

Aside from the general descriptive nature of such a model, by comparison to numerical studies new insights may be gained from inspection of the model parameters. This includes insights into the underlying dynamical properties, which are difficult to quantify and understand in depth. This means that the approach may provide a method for better characterizing and learning about the dynamical properties of cardiac fibrillation. After introducing the approach in the following sections, Sections 3.2.2f. provide a list of interpretations of the stochastic model, which may be applicable to the heart.

\subsubsection{Markov Chain Models of the Number of PS}

Two different approaches may be taken for characterizing the dynamics of PS, i.e. either quantifying the statistics for only positive/negative PS individually or the sum of both positive and negative ones. Since typically the individual count is expected to be approximately half of the total count, a large difference between the two methods is not 
expected. However, focusing on only a single chirality ignores the different mechanics of a PS entering compared to pair annihilation, and seems thus more accessible for simulations. This is because within a simulation framework with periodic boundary conditions, the topological conservation of charge means that PS always occur in pairs. $^{1}$

Here, I will derive the basic principles based on the assumption that the stochastic properties of the NPS can be described by a discrete, continuous time Markov jump model. In this context, the current state is described only by the probability distribution $P$ (NPS) and the basic transition can be split into four distinct rates or interactions which are illustrated in Figure 3.2:

$$
\begin{aligned}
C(\mathrm{NPS}) & =\frac{1}{2} r_{\text {creation }}(\mathrm{NPS}) \\
E(\mathrm{NPS}) & =r_{\text {entering }}(\mathrm{NPS}) \\
A(\mathrm{NPS}) & =\frac{1}{2} r_{\text {annihilation }}(\mathrm{NPS}) \\
L(\mathrm{NPS}) & =r_{\text {leaving }}(\mathrm{NPS}) .
\end{aligned}
$$

The factor $1 / 2$ exists since each creation and annihilation event contributes two phase singularities to the rate of change. These rates thus describe the mechanisms:

$C(\mathrm{NPS})$ : pair creation adds two PS.

$E(\mathrm{NPS})$ : one PS entering the field of view or being created at a boundary.

$A(\mathrm{NPS})$ : pair annihilation removes two PS.

$L(\mathrm{NPS})$ : one PS leaving the field of view or colliding with a boundary.

These four different events are directly accessible from experiments when using tracking methods. However, Qiao et al. [73] have suggested that an estimation from the NPS time series itself is possible even when the observation time step is large. ${ }^{2}$

One property of such a Markov model is the existance of a probability distribution $\mathcal{P}(\mathrm{NPS})$ which does not evolve over time. Given the above rates from Equation (3.9) conditions for the detailed balance in the case of stationary probability distribution $\mathcal{P}(\mathrm{NPS})$ can be derived to find it:

$$
\begin{aligned}
(C(\mathrm{NPS})+E(\mathrm{NPS})+A(\mathrm{NPS})+L(\mathrm{NPS})) & \cdot \mathcal{P}(\mathrm{NPS})= \\
& C(\mathrm{NPS}-2) \cdot \mathcal{P}(\mathrm{NPS}-2) \\
+ & E(\mathrm{NPS}-1) \cdot \mathcal{P}(\mathrm{NPS}-1) \\
& +A(\mathrm{NPS}+2) \cdot \mathcal{P}(\mathrm{NPS}+2) \\
& +L(\mathrm{NPS}+1) \cdot \mathcal{P}(\mathrm{NPS}+1)
\end{aligned}
$$

\footnotetext{
${ }^{1}$ Since there are no boundaries with which PS can collide or at which they can be created.

${ }^{2}$ The idea here is, that the measured transition matrix is given by the multiplication of $D$ shorter time transition matrices: $\mathcal{S}(\Delta t)=\mathcal{S}(\Delta t / D) \cdot \mathcal{S}(\Delta t / D) \cdots$. Thus for sufficiently large $D$ (and thus terms), the actual rate can be estimated by solving the equation for $\mathcal{S}(\Delta t / D)$ from the known $\mathcal{S}(\Delta t)$. This calculating can be performed using the fractional matrix power.)
} 
where $\mathcal{P}(\mathrm{NPS})$ is set to zero for NPS $<0$, since a negative NPS is not possible. Further, the transition probabilities can also be used to define the non-zero off diagonal elements of a transition matrix $\mathcal{S}$. Finding the transition matrix provides a convenient way to describe the system of equations derived from Equation (3.10) and to solve it using symbolical or numerical tools.

For certain functional forms of the rates and limiting to a single family of positive or negative defects $\left(\mathrm{NPS}_{ \pm}\right)$analytical forms have been derived [65, 67]. Gil et al. [65] describe that the annihilation rate in such systems is often quadratic in $\mathrm{NPS}_{ \pm}$leading to a squared Poisson distribution for $\mathcal{P}\left(\mathrm{NPS}_{ \pm}\right)$. Further, Daniels et al. [67] derive the stationary distribution for a linear leaving rate, a quadratic annihilation rate, and a constant creation and entering rate.

A general quadratic model with the constants const, $m$, and $a$ :

$$
r_{\text {all rates }}(\mathrm{NPS})=\mathrm{const}+m \cdot \mathrm{NPS}+a \cdot \mathrm{NPS}^{2}
$$

will be used in many parts for all rates in this thesis. Although limitations to the model will be used when less data is available. In this case the rates are given by:

$$
\begin{aligned}
r_{\text {creation, entering }}(\mathrm{NPS}) & =\text { const }+m \cdot \mathrm{NPS} \\
r_{\text {annihilation, leaving }}(\mathrm{NPS}) & =m \cdot \mathrm{NPS}+a \cdot \mathrm{NPS}^{2}
\end{aligned}
$$

These limitations disregard the quadratic term for the entering and creation rates, and the constant term for the leaving and annihilation rate. In both cases these terms are theoretically not expected to be important or, in the latter case, expected to be zero ${ }^{3}$.

In the results Chapter 6 the stationary distribution $\mathcal{P}(\mathrm{NPS})$ for the rates described by Equation 3.11 will be found by symbolically or numerically solving Equation (3.10) with a sufficiently high maximum NPS. Further properties of the Markov model system can be readily estimated numerically by simulating the system using the Gillespie algorithm [75].

\section{Alternative Models for the Stationary Distribution}

As an alternative to the Markov model derived above the following models will be considered for the description of the stationary probability distribution of the NPS:

- As a generic model and approximation expected to be correct for large enough NPS a Gaussian model can be fitted to the data. The Gaussian model has been used by Sugimura and Kori [76] for numerical simulations to estimate the time until the chaotic dynamics stop - a property commonly seen in such systems [77]. Additionally to a Gaussian probability distribution the authors introduce a transition rate from the state with one PS to the actual quiescent

\footnotetext{
${ }^{3}$ The annihilation/leaving rate has to be zero for NPS $=0$. Any negative rate arising due to fitting or extrapolation is thus replaced with zero.
} 

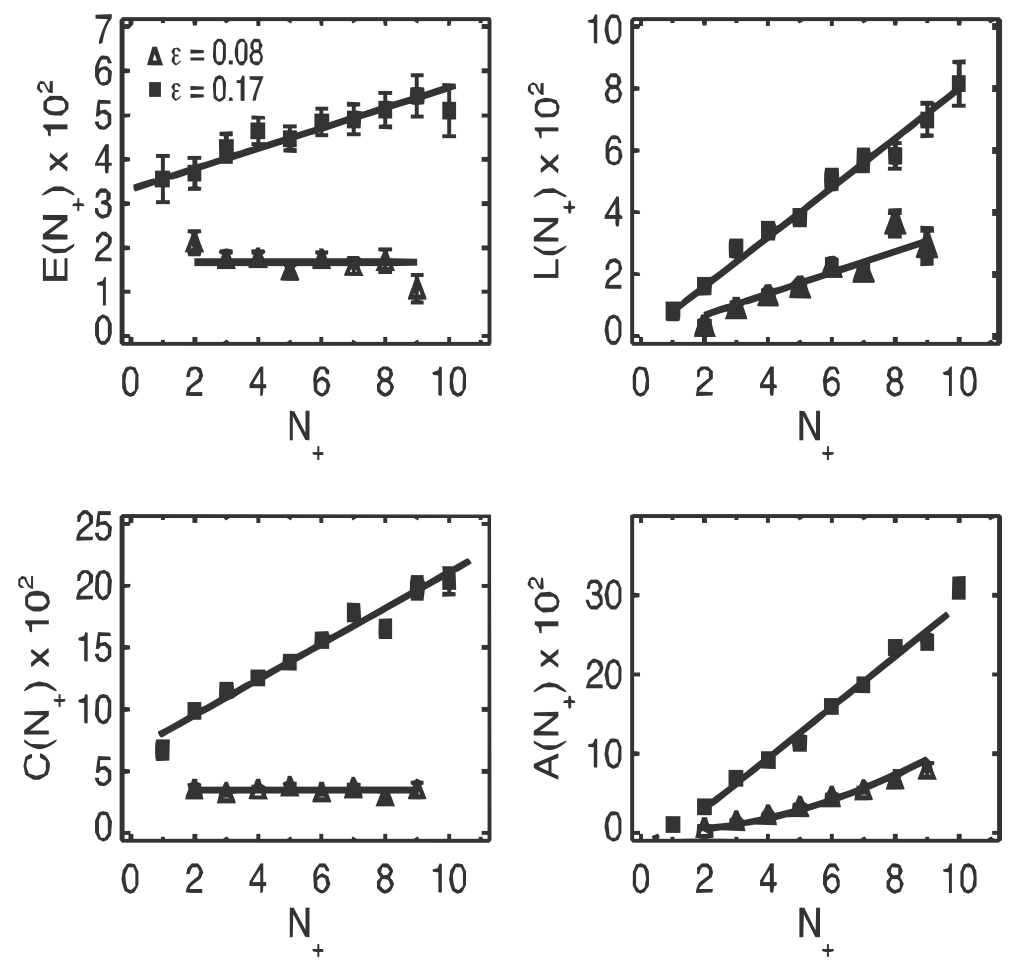

Fig. 3.3 Entering $(E)$, leaving $(L)$, creation $(C)$ and annihilation $(A)$ rates as function of the positive defects in an inclined-layer $\left(30^{\circ}\right)$ convection as presented by Huepe et al. in [70]. $\epsilon$ is defined by the temperature ratio $\epsilon=\Delta T / \Delta T_{c}-1$ and is a parameter tuning the chaos in the system. Reprinted from [70], with the permission of AIP Publishing.

state where no activity is left. The approach of using a Gaussian distribution is motivated by the central limit theorem.

- A Markov model that only allows for a single step increment or decrement in the NPS. A comparison with such a model highlights the importance of pair interactions for the dynamics.

\subsubsection{Properties and Interpretation of the Transition Rates}

In the previous section a stochastic Markov model for the NPS and its stationary distribution was presented. This Markov model thus makes predictions about the distribution of the NPS found in the experimental system and can be compared to it. Considering the complex dynamics of VF the correctness of such prediction is not guaranteed. For example periodic fluctuations could mean that a reduction to only the NPS is not emough to sufficiently describe the current state. However, the success when applied to other experimental systems as well as to numerical simulations suggest that such a model may be applicable to fibrillation.

The confirmation that the correct stationary probability can be estimated from the rates is important. Furthermore, the properties of the rates of change of the Markov 
model and how these vary with the NPS may be used to characterize and gain insights into the dynamics.

Figure 3.3 presents the rates for a single charge as found in an inclined-layer convection experiment by Huepe et al. [70]. In this experiment $\epsilon=\Delta T / \Delta T_{c}-1$ is a parameter of the system controlling how chaotic it behaves. Some general properties are visible from these measurements and were, in other studies, numerically and theoretically motivated:

1. For small $\epsilon$ both the entering rate and creation rate seen in in Figure 3.3 are approximately constant or slightly negative. This property is shared with many simulations of two dimensional models [74]. Qiao etal. [73] find also only a slight linear contribution in a two dimensional Belousov-Zhabotinsky reaction ${ }^{4}$, although St. Yves et al. suggested that noise could contribute in such a way [74].

2. For large $\epsilon$ a clear linear contribution to the entering and creation rates appears. In [72] Davidsen et al. show numerically that such a positive linear contribution occurs in, and may indicate, three dimensional dynamics. One reason for this can be negative filament tension. More PS in the system are related to more filaments and if negative filament tension is the mechanism of creating new PS, it is expected that more are created when more filaments already exist. In a later paper [74] they show that this can occur more generally when introducing a third dimension due to how filaments interact with the surface. The authors of [74] also points out an example of a system where an increase happens in two dimensions already. ${ }^{5}$ Yet, the rate may be an indicator of dimensional effects and is expected to have a strong linear component if the negative filament tension is the dominating effect driving the fibrillation. This may be interesting for the heart, where the dimension of the dynamics or the contribution of the negative filament tension is not well known.

3. The leaving rate can be estimated as a linear function of the NPS which is also found in other numerical and chemical systems cited before. Such a behaviour can be motivated by the assumption of randomly moving short range interacting defects.

4. The annihilation rate is first quadratic. When $\epsilon$ is increased, it becomes linear or has a large linear contribution. If defects are only weakly or short range interacting, a quadratic rate may be expected due to a higher likelihood of collisions as the density increases. As noted in [70, 78] a linear contribution indicates that $P S$ are interacting strongly and may for example commonly annihilate together with the same PS with which they were created.

To summarize, one of the main interest for studying the Markov model of the NPS over time, are the properties of the derived rates. These may serve as an indication both for the dimension on which the dynamics occur, as well as for whether or not

\footnotetext{
${ }^{4} \mathrm{~A}$ class of oscillatory chemical reactions capable of showing spiral waves.

${ }^{5}$ Which may be more similar to the one shown in Figure 3.3
} 
spirals are strongly interacting. Maybe more importantly the rates can provide an additional way to characterize the dynamics of the arrhythmia. This could help to distinguish different mechanisms underlying an arrhythmic episode.

\subsubsection{Properties and Interpretation of the Stationary Distri- bution}

Additionally to finding a general model and to validate the Markov model as an approach to characterization, the stationary probability distribution, as measured in the experiment or derived from the above introduced rates, may be used to interpret the properties of the system.

The main interpretation here will be the one of a spontaneous stop of fibrillatory activity when no phase singularities are left in the system. ${ }^{6}$ The phenomenon of selftermination is well known both for the heart as well as for general excitable media. There, depending on the system size, chaos can suddenly stop and reach the stable quiescent state without any spirals [77]. The probability of this transition and the average time until it occurs has been estimated from the phase singularity probability distribution in simulations by Sugimura et al. [76]. They did so by introducing an additional state and transition to this state, where activity is stopped and the model will remain perpetually. With an additional Gaussian assumption they were able to describe the average time of the transition to the quiescent state as a function of system size. While this exact method cannot be applied easily to experimental data, this suggests that, also in the heart, it is possible to use the derived distribution to assess how likely it is for self-termination to occur.

Although not used in this thesis, one further observation discussed by Carsten Beta in his $\mathrm{PhD}$ thesis [79, p.62] and shown by Hildebrand et al. [80] is that the width of the distribution may provide information about how close the system is to a non-chaotic state. In the heart, a transition from tachycardia to fibrillation may be expected to follow similar rules as the bifurcations, that lead from stable spirals to chaotic behaviour, seen in theoretical systems. This would suggest that the width of the distribution $\mathcal{P}\left(\mathrm{NPS}_{+}\right)$may provide hints on how close the heart is to tachycardia. ${ }^{7}$ In tachycardia the NPS is not expected to fluctuate and in a fibrillation, which is close to a tachycardic state, the fluctuation of the NPS could be expected to be small leading to a narrow probability distribution.

\footnotetext{
${ }^{6}$ The NPS $=0$ state, or a quiescent state, is a so called absorbing state if the Markov model never leaves it once it is reached.

${ }^{7}$ For this type of analysis no modelling itself should be necessary.
} 



\section{Chapter 4}

\section{Video Processing and Data Anlaysis Methods}

In this chapter, I will describe the full video data analysis pipeline used to produce the results shown in this thesis. It will go through all of the data analysis steps and explain why they are performed. Since my processing pipeline slightly differs from other approaches often used for the analysis of cardiac optical mapping data it will be discussed in more detail. Almost all of the analysis performed in this thesis is based on the robust estimation of when upstrokes, the time of local activation, occurred. While this is not the first step, it is the most central one to all video analysis, including the phase singularity (PS) tracking, which is described in Section 4.2. The general analysis procedure is as follows:

1. General (pre-)processing of the video data:

- Manually create a mask to limit the view to the ventricles.

- Perform basic cleanup of the video data and merge the videos from all four cameras into one video.

- Spatially smooth the image using a Gaussian smoothing kernel.

- Rescale the amplitude of each pixel in the video to simplify a homogeneous data analysis.

2. Find times when activations/upstrokes occur by employing a specially defined "upstroke-like" kernel (Sec. 4.2).

3. Perform video analysis, often making use of these excitation times:

- Map the activation times and analyze them.

- Extract the cycle lengths (CL), the time between upstrokes.

- Analyze the action potentials.

4. Make use the of the excitation times to find and especially track phase singularities (PS)

5. Analyze PS properties.

Even with detailed description of the data processing, it is difficult or time consuming to reproduce results in detail. Therefor, the code used to analyze the data will be made available upon request. 
a)

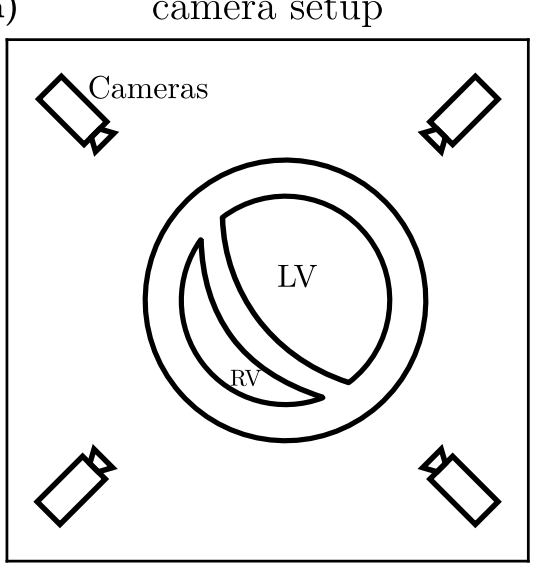

c) fractional fluorescence

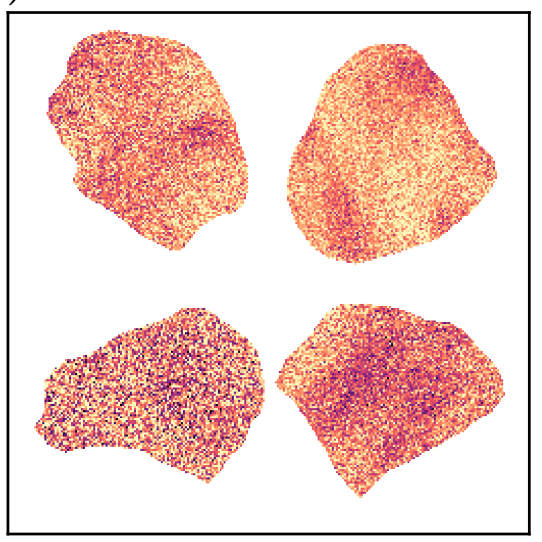

e)

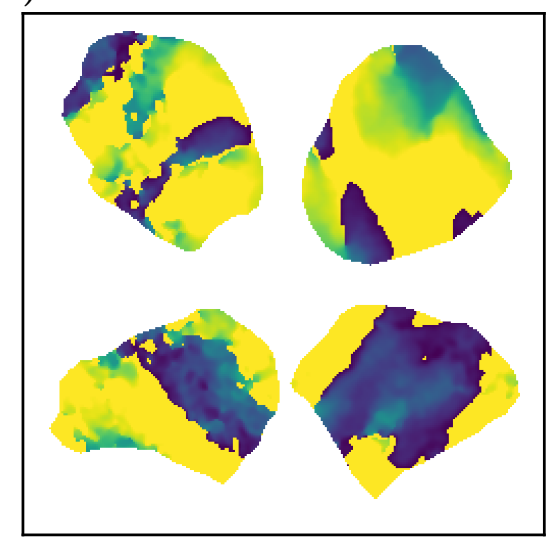

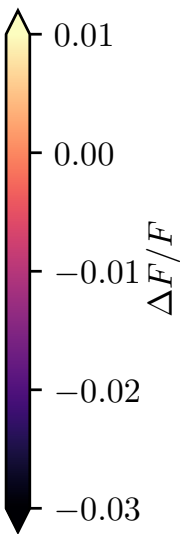

$-0.03$

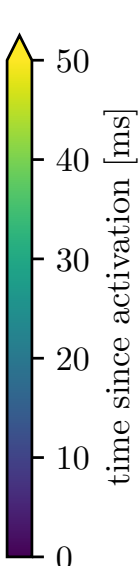

b)

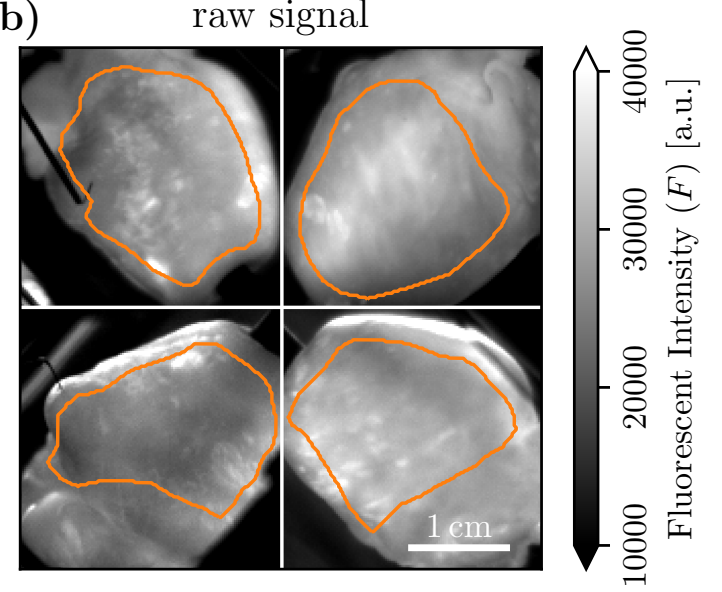

d)
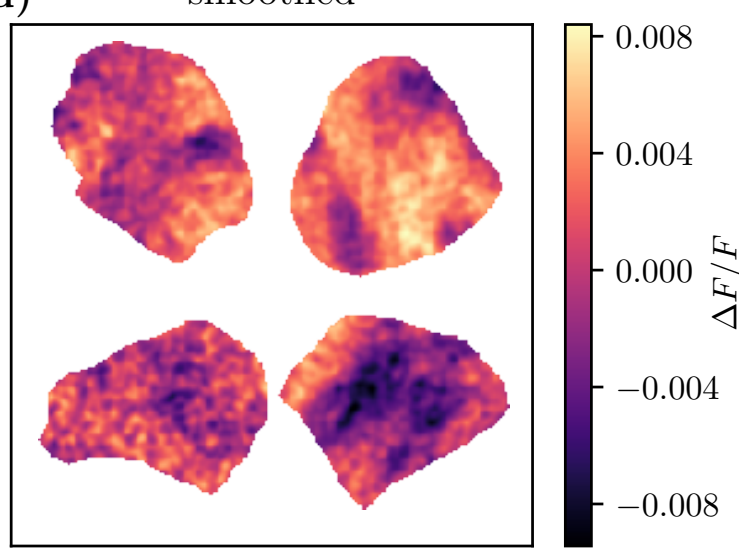

f)

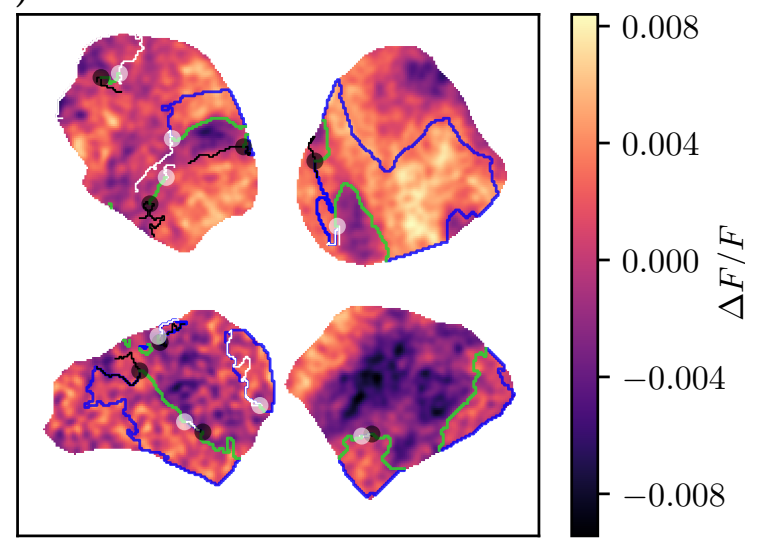

Fig. 4.1 Overview over the main steps of the video processing. Caption continued on next page. 
Fig. 4.1 (continued) a) Sketch of the setup with the heart. The view is from below the heart (from apex) which places the apex being in the centre in the following panels. b) The raw fluorescence signal of a rabbit heart stained with di-4-ANEPPS during an episode of ventricular fibrillation. The orange line indicates the mask used to limit the analysis to the ventricles and regions of good optical quality. c) Same as (b), but with the mask applied and the signal normalized to the fractional fluorescence. The activation waves become faintly visible as dark regions. d) Same as (c), but including the spatial smoothing step. e) Result of finding the activation time. It shows the time since a pixel was last activated in milliseconds. Dark blue areas were activated very recently. f) The same as (d) but including PS as black/white dots indicating chirality, wavefronts as green lines, and fine black/white lines showing the movement of the PS within $\pm 20 \mathrm{~ms}$. The green and blue lines together encircle areas that will be activated in the next $20 \mathrm{~ms}$. This is based on the analysis in (e) and explained in detail in Section 4.4. Sample traces from (c) and (d) can be found in Figure 4.2 (a). Figure 4.2 also explains the step from (d) to (e) in more detail.

\subsection{Preprocessing of the Video Data}

\subsubsection{Manual Creation of Masks}

Before any detailed analysis is possible, it is necessary to restrict the analysis of the video data to the areas of interest and with sufficient quality. While our group has experimented with automatic procedures to achieve this, manual mask creation is quick and reliable. The general method to create all these masks was as follows:

- Perform temporal filtering and play the video to observe the dynamics.

- Identify the contours of the ventricles. It is important to ignore both the atria as well as fatty tissue close to the atria where the signal quality is poor.

- The cameras have overlapping fields of view and in later steps spatial filtering is applied. Because of this the mask is chosen slightly smaller than the actual contours of the heart. This also mitigates possible artifacts which are often larger close to the contours of the heart.

Note that the mask is always applied after and not before the spatial smoothing step.

\subsubsection{Basic Video Preprocessing}

Unfortunately, due to technical issues related to the Photometrics EMCCD cameras, the raw video data has to receive very basic preprocessing. The first step of this is the removal of the first few frames from each movie because they are overexposed. Second, all cameras are triggered externally every $2 \mathrm{~ms}$ which is inconsistent with the cameras internal clock and causes slight differences in actual exposure time at regular intervals. This means that every five to six frames the frame has a slightly lower exposure time. 
Fortunately, the change is easy to detect and corrected since the difference in exposure time can be calculated from the internal clock. ${ }^{1}$

\subsubsection{Spatial Smoothing}

To reduce the noise, it is necessary to apply spatial filtering. The two main methods commonly employed in the cardiac sciences are either a square averaging or Gaussian kernel smoothing [81-83]. In this work, Gaussian kernels are used throughout for spatial smoothing. Due to the difference in signal quality and analysis needs, here, two different parameter sets are employed. For analysis of the activation times, the Gaussian smoothing parameter $\sigma$ is set to $\sigma=1$ pixels. For the analysis of all other video data, generally $\sigma=1.75$ was employed. These values corresponds to roughly $\sigma \approx 0.14 \mathrm{~mm}$ and $\sigma \approx 0.35 \mathrm{~mm}$.

\subsubsection{Rescaling to the Fractional Fluorescent}

The total brightness at a specific location is dependent on the local staining and illumination. Since the application of thresholds will be necessary in the following steps, it is useful to normalize the video. Here, this is achieved by dividing each pixel by its average fluorescence value $F$. This transforms the pixel value into the fractional fluorescence $\Delta F / F$, where $\Delta F$ is the change due to the electrical excitation (compare Sections 2.2 f.). ${ }^{2}$

\subsection{Detection of Activation Times}

The detection of upstrokes or activation times is central to data analysis tasks when studying the heart. Commonly two different approaches are used. When analyzing arrhythmia, temporal filtering with a following mean or median threshold is applied. For data with clear upstrokes - when there is no arrhythmia - the upstroke may be defined by using a threshold or as the point where the derivative is largest.

Here, a slightly different method is used. The approach is similar to typical edge detection methods used in computer graphics [84] and has been briefly shown in [85]. The main idea is to employ a step or activation like kernel function and perform the convolution (or correlation) with the original signal. This procedure is summarized in Figure 4.2. Panel (a) shows an example time series of a pixel trace from a VF episode including the times that are finally detected as an activation. Panel (b) shows the kernel employed and Panel (c) the result of correlating ${ }^{3}$ the original signal with the

\footnotetext{
${ }^{1}$ This has been done by Johannes Schröder-Schetelig. To achieve correction with a slightly higher precision, I use a linear program with the constraints that every 5th or 6th frame is corrected. The linear program is implemented using the CVXOPT python package.

${ }^{2}$ The resting state is actually the highest value, so that $F$ is only an approximation of the base value. However, the difference between the two is small since $\Delta F$ is small.

${ }^{3}$ Meaning a convolution operation with (b) flipped.
} 
a)

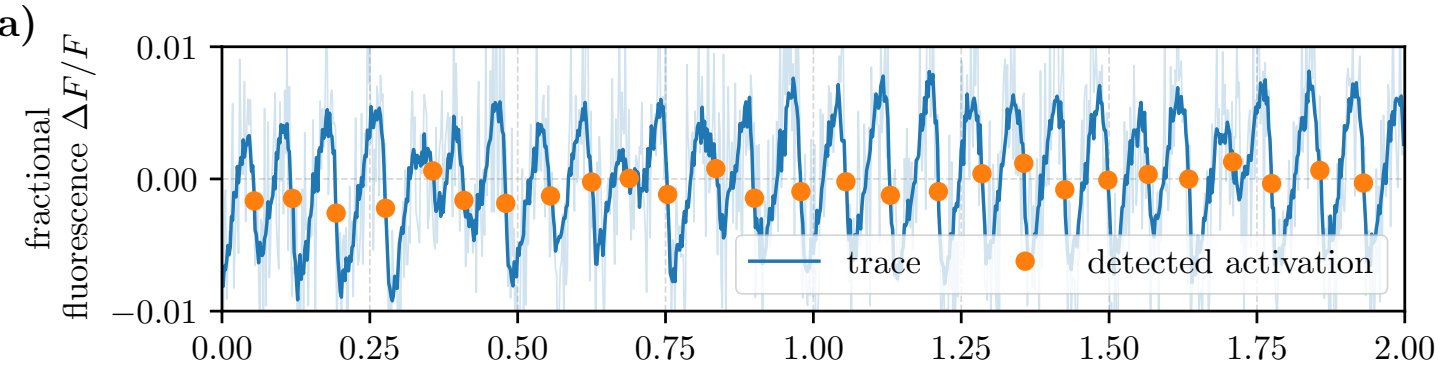

b)

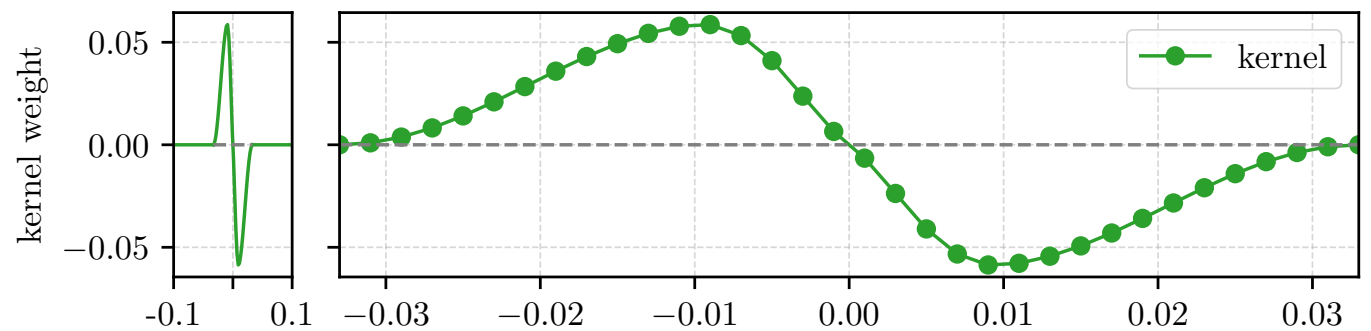

c)

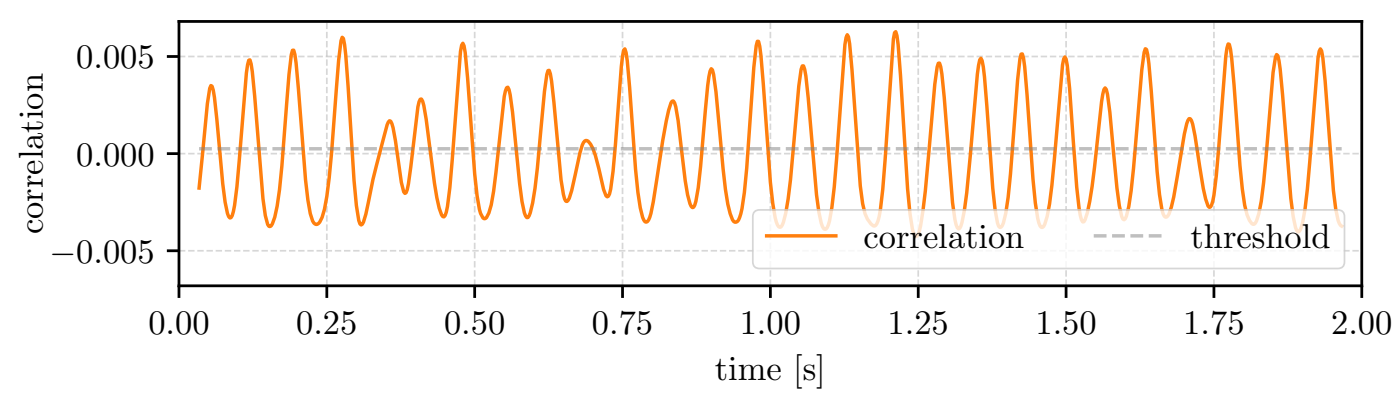

Fig. 4.2 Example of the method for detecting the upstrokes during an arrhythmia. a) An example time series from a single pixel during VF, with the final excitation times indicated. "Upstrokes" in the action potential point downwards in the fluorescent intensity. The thick line shows the trace with spatial smoothing applied. The same pixel trace without spatial smoothing is indicated in light blue. b) The kernel function used to identify steps/upstrokes in the original signal. In the left panel scaled to match (a) and enlarged along the time axis in the right panel. c) The result of correlating the original time series from (a) with the kernel in (b). The local maxima mark the upstrokes - as shown in (a) - with very small upstrokes removed. Due to particularly short action potentials in some of the experiments, a short kernel was used. Further, it worked better to err on the lower side for the threshold value. 
kernel. The theoretically best choice of the kernel function is complex and depends on the signal [84]. The kernel used here is based on the Tukey windowing function and gives good results in practice although it is not optimized. Python code to generate this kernel can be found in the Appendix B.1 (p. 158).

Below, the necessary steps and parameters are described in more detail:

1. The choice of the kernel parameters.

2. The identification of upstrokes from the convolution.

3. Additional processing performed.

For all data analysis shown in the thesis the same kernel parameters as shown in Figure 4.2 were used. The general parameters decide on how long the kernel should be and how smooth the step/central part is ${ }^{4}$. With better data quality both can be chosen shorter to avoid systematical errors, while larger parameters make the result more robust. In general, however, the total length should not be longer than the action potential duration. The analysis results are however generally very robust to changes in these parameters. The parameter for the action potential duration was chosen to be short, due to the short action potentials observed in the Pinacidil experiments analyzed later.

Using this kernel, it is now possible to calculate the smooth signal seen in Figure 4.2 (c) which reaches a maximum when the kernel correlates well with the signal. From this correlation signal the upstrokes can now be identified as local maxima. To suppress too many misidentifications due to noise, a threshold slightly above zero is used. This threshold is set to $2.5 \cdot 10^{-4}$ for the arrhythmia videos and $7.5 \cdot 10^{-4}$ otherwise. While the threshold is small, it is chosen to reduce the number of incorrect detections when almost no signal is present during the arrhythmia. The threshold used when no arrhythmia is present is high enough to suppress most of the otherwise spuriously detected activations.

The correlation result is robust and smooth. Thus, to improve the activation time definition to below one frame a quadratic interpolation around the maximum value is used. While care should be taken not to interpret the results as being more precise than the intrinsic camera exposure time of $2 \mathrm{~ms}$, it works well in practice.

After the activation times are detected, two additional processing steps are applied:

1. If detected upstrokes occur very close (less than $42 \mathrm{~ms}$ from each other), the one with the higher correlation value is chosen. This makes use of the assumption that there is a refractory time inhibiting very fast activations. During activation map and pacing analysis, with known much slower activation, this parameter was increased to $200 \mathrm{~ms}$.

2. For the analysis of arrhythmia better results were achieved with a lower rather than a higher threshold. Additionally, however, when an activation is detected

\footnotetext{
${ }^{4}$ It should be noted, that due to the finite propagation velocity and intrinsic limitations, the upstrokes are smoothed out even without any filtering. Further, the kernel should be smoother than the signal to reduce the effect of noise.
} 
without sufficient neighbours also being activated shortly after, this activation is ignored.

In general the signal processing using the kernel is very robust, although slight systematic errors may arise for the combination of poor data and short action potentials. However, similar shifts will also occur for any other temporal filtering technique and manual inspection for the activation map analysis showed no visible shift for the detected upstroke compared to the true upstroke. Thus, this approach worked reliably for all data analysis purposes presented here.

\subsection{Further Analysis}

Equipped with the previous tools and results, it is now straight forward to calculate many of the interesting values describing the activation patterns of the heart. This includes activation map analysis and the analysis of cycle lengths. It is also used as a basis for the PS tracking described in Section 4.4 .

\subsubsection{Activation Map and Time Analysis for Quiescent Tissue}

Activation maps describe how a shock, during the quiescent state of the heart, causes excitation that propagates and finally activates the whole surface. To visualize this activation it is customary to plot the the time until activation $[4,18,20,63]$. Since this time changes with the applied shock voltage, a number of increasing shock voltages are always measured at a CL faster than the sinus rhythm ${ }^{5}$. Each shock voltage is also repeated multiple times for three reasons:

1. The heart is still in sinus rhythm, so it is not fully quiescent. Multiple shocks are necessary to "win" over the sinus rhythm, especially at low voltages.

2. When increasing the shock voltage a short transient can occur, so that the first shock may have a different activation time.

3. Multiple shocks allow to see variations and estimate errors.

Since the recording program runs both the shock protocol and the cameras simultaneously, the exact time when a shock occurred in a video can be calculated. Using this information and the times when excitation occurred - as described in the previous section - the time until activation occurs is calculated for every pixel.

For the activation maps shown in this thesis, the time for each pixel is given as the median over the the shocks of the same voltage. The activation time is given as the time after the shock until $95 \%$ of the area is activated. Occasionally the sinus rhythm can disturb the measurement causing a large variability in the activation times. In rare cases also an arrhythmia occurred causing unusable measurements. Thus to filter out incorrect events or fluctuations, the following rules are applied:

\footnotetext{
${ }^{5}$ Generally 300 ms was used.
} 
1. The result of the first shock is always ignored.

2. If the heart was largely activated within $100 \mathrm{~ms}$ before the shock the value for this shock is discarded. This is because the activation from the previous shock would have happened much earlier.

3. Shocks with an activation time outside a 1.5 inter quartile range compared to the other shocks of the same voltage are ignored.

4. If less than half the points remain, the measurements for that voltage are discarded.

These rules allow the analysis of a large amount of activation maps without manually inspecting where sinus rhythm disturbed the measurement.

In very rare cases a natural alternating pattern occurs for the activation times, these can sometimes be lost due to the filtering. However, typically the variability of the activation time measurements from individual shocks is within $\pm 0.2 \mathrm{~ms}$, making the analysis used extremely robust.

\subsubsection{Cycle Length}

One interesting and commonly analyzed measure of a cardiac arrhythmia is its activation frequency. The cycle length (CL) is directly related to the frequency and thus can be analyzed analogously. To find the most typical CL as reliably as possible, all detected cycle lengths of a video, defined as the time between two upstrokes, are pooled. Then a kernel density estimator is used to find the dominant CL. The kernel density estimator used was scipy.stats.gaussian_kde from the SciPy package [86].

Since different estimations give slightly different results, the Appendix A.4 (p. 150) includes a comparison of how a spatial average (over the average for each pixel) behaves compared to the dominant CL reported in this thesis. This appendix also compares the dominant CL to the period as detected from the ECG by fitting a sinus to a one second segment ${ }^{6}$.

\subsubsection{Action Potential Duration}

The action potential duration (APD) describes for how long the cardiac cell is depolarized or excitated. This depends on the exact level $V_{\mathrm{m}}^{\text {thresh }}$ of the membrane potential $V_{\mathrm{m}}$ at which the APD is measured. A common value is for example the $\mathrm{APD}_{90}$ with the $90 \%$ denoting the duration for which the action potential is within $90 \%$ of its maximum value (compare also Figs. 1.2 and 4.3):

$$
V_{\mathrm{m}}^{\text {thresh }}=\min \left(V_{\mathrm{m}}\right)+\left(\max \left(V_{\mathrm{m}}\right)-\min \left(V_{\mathrm{m}} \cdot\right)\right) \cdot(100-\mathbf{9 0}) / 100
$$

Two different methods for calculation are used:

\footnotetext{
${ }^{6}$ The approach of the fit was developed by Annette Witt and improved by me for the live data analysis task necessary in our defibrillation experiments.
} 
1. Based on the PseudoECG - the average fluorescent signal over a whole camera with the assumption that excitation is fast enough during sinus rhythm.

2. Based on "folding" and averaging action potentials during the arrhythmia or pacing protocols. This is done by aligning action potentials based on the excitation times found previously. Such folding approaches are more often used when the folding period is already known from a pacing frequency. This alignment then allows to take an average signal over all action potentials detected for each pixel. Then, the APD is directly estimated for each pixel giving a map. This method is used in Chapter 7 for pacing events. It is also used to calculate an estimated APD during the arrhythmias analyzed in Chapter 5. Thus, the APD values given for arrhythmic data should be seen as an APD of the averaged action potential rather than a true APD value. However, the definition of an APD during arrhythmia is otherwise difficult and this approach provides an estimation. When reporting the APD for a VF episodes, the APD is here given as the spatial average over the APD map.

\subsubsection{Triangularity Index}

For the Pinacidil experiments shown in this thesis Daniel Hornung developed the triangularity index. This index aims to capture the changes in the action potential shape commonly associated with ischemia, i.e. the reduction of the plateau phase in the action potential towards a very narrow and pointy shape (compare Sec. 1.6). While the APD captures such changes to some degree, multiple APD levels would be necessary to capture the change of the shape itself. Another definition of triangularity has been previously used by Hondeghem et al. [87] by using the difference between the $\mathrm{APD}_{30}$ and $\mathrm{APD}_{90}$. They have found it to be a good predictor of the pro-arrhythmic effects of drugs.

Here, the goal is to capture the shape in a way that is more independent of the APD. Thus, the triangularity index is defined to quantify the deviation from a triangular action potential shape. Figure 4.3 shows three example traces of action potentials and their corresponding triangularity index at an $80 \%$ level $\left(\Delta_{80}\right)$. The triangular shape indicated is given by the maximum point during the action potential and a threshold value defined in the same way as the APD level. The green area in the figure indicates the deviation of the action potential from the triangle. The triangularity index is then defined as the ratio of the integral over this deviation and the triangles area. A large value thus indicates a prominent plateau phase - associated with the healthy heart and a value around or below zero translates to a narrow or pointy action potential associated with the ischemic condition.

The examples in Figure 4.3 are based on a single video containing multiple pacing events. For this analysis multiple action potentials are "folded" and averaged as explained for the APD maps. For the overview plots, instead the PseudoECG is used as the basis for finding the triangularity index. 

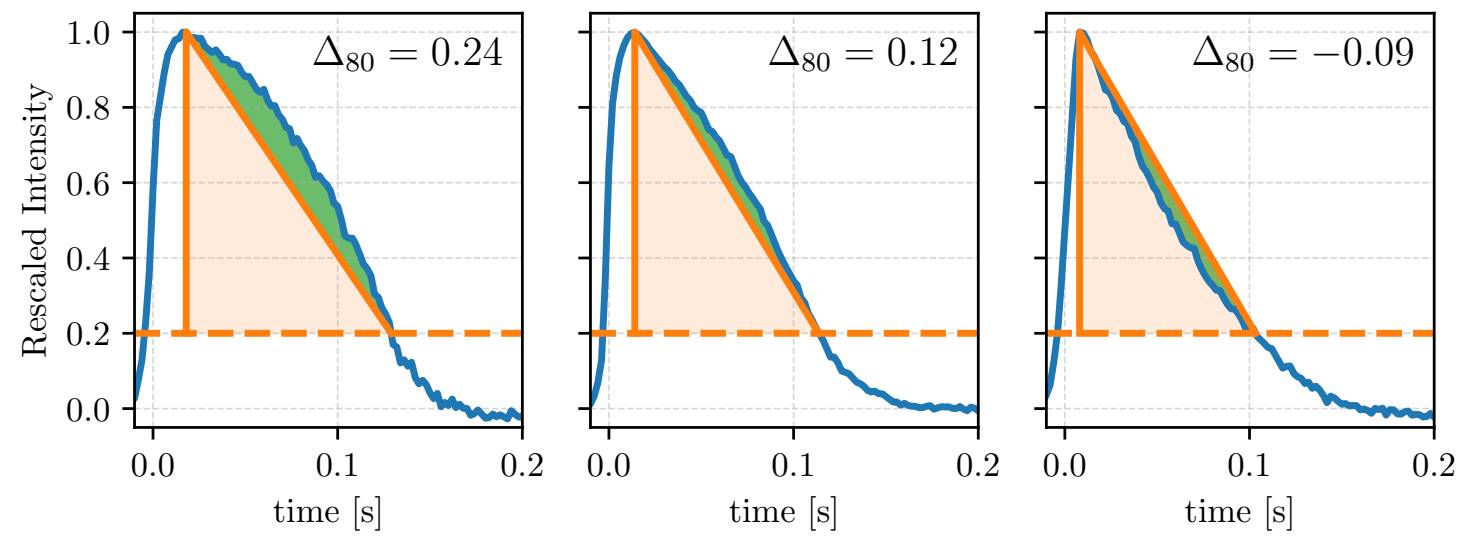

Fig. 4.3 Example of three action potentials and their triangularity index. All examples come from a recording paced at $300 \mathrm{~ms}$ on the 2017-10-05. The blue lines show the action potential scaled from zero to one. The horizontal dashed orange line shows the level corresponding to an $\mathrm{APD}_{80}$ or $\Delta_{80}$. An orange triangle extends from the maximum point in the action potential to the downstroke crossing the $80 \%$ level. The area of the triangle is indicated in orange. The deviation of the action potential from the triangular shape is indicated in green. The $\Delta_{80}$ is now given by the integral over the green area divided by the triangle area.

\subsection{Phase Singularity Identification and Tracking}

As already shortly reviewed in the introductory Section 1.2.1 cardiac arrhythmia is in most cases thought to be driven by rotors. During ventricular fibrillation, electrical excitation waves propagate continuously through the heart in a spiral like fashion. At the centre of each spiral wave exists a point around which the wave rotates. Furthermore, locally the heart goes through an oscillation like activation which can be associated with a phase. This phase will change continuously in time, however, in space it is continuous only in most places. The centre of each rotation contains a discontinuity much like a spiral staircase always has a pole at its centre. This point ${ }^{7}$ is the phase singularity (PS) and a hallmark of cardiac arrhythmia. In earlier cardiac literature other names, such as pivoting points [28] or wave breaks [88] have been used. These names will be used synonymously here, because conceptually they both describe the spiral cores and differ only in how the phase is defined. These differences will be discussed below.

This chapter gives a short overview over different methods of defining PS and identifying them. It further describes the approach developed and used for the data analysis in this thesis.

\footnotetext{
${ }^{7}$ The identification as a single point is practical but not necessarily correct. Indeed, linear reentries are common in cardiac tissue and a PS is "smeared" out in this case with the phase map being noncontinuous on a line.
} 


\subsubsection{Overview}

Phase singularity analysis can be separated into three parts, though, depending on the algorithm used, each may not be obviously separated:

1. defining the phase map

2. identifying the phase singularities

3. tracking phase singularities.

Although none of these steps may be expected to be particularly difficult, in practice each one poses its own challenges, as evidenced by continuing research in developing techniques to analyze PS and the multitude of methods employed in practice. Many of these methods, not including additional variations in tracking methods, are listed in the Appendix C.2 (p. 161). The main reasons for such difficulties are:

1. Video noise will cause variations in the phase.

2. Discrete pixels mean that phase gradients become phase differences. However, unlike phase gradients, phase differences are ill defined. For example, a phase difference of $-\pi$ is identical to one of $\pi$. By definition, however, close to a PS the gradient in the phase map must become large. Such large gradients mean that large phase differences are likely to be found. These large differences can then become problematic since their correct sign is unclear.

3. Complex activation patterns additionally complicate tracking by causing fast moving PS.

In the following sections I will briefly review some of the methods used for finding the phase map and PS. Then some of the issues and limitations will be discussed in more detail. Finally, the method of phase singularity identification and tracking used in this thesis will be presented and the different analysis steps described.

\subsubsection{Typical Definitions for the Phase}

In the Section 1.2 the local oscillator behaviour was shown exemplarily for the BärEiswirth model in Figure 1.3. In such a two variable model, a phase may be defined directly based on the two variables $-u$ and $v$ in the Bär-Eiswirt model. During the dynamics, the local oscillators rarely come close to the unstable point at $u=0.65$ and $v=0.45$ (compare Fig. 1.3) and thus the phase was defined as the angle around it.

\section{Defining the Phase using Embedding}

In simulations heart cells are often described by more than two variables while in experiments commonly only one variable - the membrane potential - is recorded. However, for defining a phase a second variable is necessary since an angle needs to be defined. Thus, the second variable needs to be reconstructed. This can be achieved by using the delay embedding technique from nonlinear time series analysis [89]. Delay 
embedding defines a time delay $\tau_{d}$ and then uses not just the membrane potential as a function of time $V_{\mathrm{m}}(t)$ but also the value at a delay $V_{\mathrm{m}}\left(t+\tau_{d}\right)$. The value for $\tau_{d}$ has to be chosen based on the typical oscillation period and APD. $\tau_{d}=25 \mathrm{~ms}$ has for example been used in [38]. After defining two variables, the phase can be found as before. For this the mean value $\left\langle V_{\mathrm{m}}\right\rangle=1 / T \cdot \sum V_{\mathrm{m}}(t)$ may be used to give the formula:

$$
\varphi=\operatorname{atan} 2\left(V_{\mathrm{m}}\left(t+\tau_{d}\right)-\left\langle V_{\mathrm{m}}\right\rangle, V_{\mathrm{m}}(t)-\left\langle V_{\mathrm{m}}\right\rangle\right)
$$

\section{Defining the Phase through Wavebreaks}

Another method of defining phase singularities (or wavebreaks) is to find the points where the wavefronts and wavebacks connect [28]. Here the wavefront is defined as the area that was just activated while the waveback is defined as the area where the action potential just dropped again. Such points where wavefront and -back meet will always exist for a stable spiral reentry. Effectively, it can be thought of as defining four distinct phases based on the comparison of the active areas in two consecutive (or time delayed) frames:

1. Activation phase: If a region was not active in the previous frame but is active in the current frame.

2. Active phase: If a region is considered active both in the current and the previous frame.

3. Refractory phase: If a region was active in the previous frame but is not active in the current frame.

4. Excitable phase for regions not active in either frame.

If each of these phases is assigned a phase value differing by $\pi / 2$ from the previous one this also defines a phase map similar to those of other approaches.

\section{Defining the Phase via Hilbert Transform}

Another method to overcome the difficulty when only a single variable $u$ - such as the membrane potential - is measured is to use the Hilbert transform of $u$ :

$$
H_{u}(t)=\frac{1}{\pi} \int_{-\infty}^{\infty} \frac{u(\tau)}{t-\tau} \mathrm{d} \tau
$$

In the Fourier domain the Hilbert transform causes a phase shift by $90^{\circ}$. It thus allows the definition of the analytic signal:

$$
\begin{aligned}
u_{a}(t) & =u(t)+i \cdot H_{u}(t) \\
& =\left|u(t)+i \cdot H_{u}(t)\right| \cdot e^{i \cdot \varphi} .
\end{aligned}
$$

With the value for $\varphi$ defining the phase. 
Since the Hilbert transform requires a stationary signal it typically requires a prior trend removal step by removing the mean or using a highpass filter. Sometimes more complex methods are used in an attempt to improve the behaviour for complex signals (compare also the notes in App. C.2).

\subsection{Problems and Limitations of Singularity Iden- tification}

While PS identification is relatively simple for stable spirals where the phase map is sufficiently smooth, problems arise as soon as the behaviour is more complex. Here, I wish to showcase an exemplary problem which can arise due to more complex wave structures and discuss a limitation regarding the slowest detectable propagation speed.

\subsubsection{Complex Propagation Patterns}

Far from any spiral core, wave propagation is typically regular. Such regular propagation is associated with a smooth phase map. For stable spiral cores, this means that the phase differences calculated when identifying phase singularities are well defined. For stable spirals large phase differences mostly arise close to the cores where they are expected. However, when spiral cores are moving quickly complex wave patterns can arise which can cause large phase differences - those close to $\pm \pi$ - between neighbouring pixels. Here are two examples of such wave patterns:

1. Linear reentries, which are common in cardiac dynamics, have a long/smeared area where phase differences (derivatives) are large. An algorithm must place the PS at some point in this area. However, in an area where large phase differences occur even small amounts of noise will lead to the wrong identification of PS.

2. A fast wave colliding with a leading waveback stops and must have a large phase difference along the line of block. Such difference may remain for a longer period of time. However, along the line where the wave is stopped short living PS pairs may be created and collide with each other. These short lived PS can be created even without noise, but noise will exacerbate the issue.

Further, artifacts due to pixels of low quality or with misidentified phase can create PS when a wave passes them. Many of the PS created through noise or complex wave patterns are of a transient nature and have little or no effects on the actual dynamics. A simple partial wave block may create a large number of short lived PS, which, after taking an average of the NPS, may suggest an increased complexity.

\subsubsection{Minimum Propagation Speed}

A second consideration concerns the required spatial and temporal resolution. To be able to estimate phase differences, the absolute phase differences between neighbouring 
pixels should be below $\pi$. Thus, a slowly propagating wavefront must move by one pixel in less time than it needs for the local phase to advance by $\pi$. Any slower movement will create a large phase difference and - at least from the perspective of the phase map - lose the information about which direction the wave moves. Since the local dynamics goes through all $2 \pi$ phases within one CL, the propagation speed should be faster than one pixel in half the CL.

Except for very low resolution methods, this consideration should often not matter in practice. However, it is mentioned here because in the method used in this thesis, it is used to justify the assumption that a wave has a minimum propagation speed. For long APD and CL this limitation would give a much slower propagation speed. However, in practice cardiac dynamics has a slower propagation speed when CL are shorter.

\subsubsection{Definition of the Phase in this Thesis}

In this thesis, the phase will be defined based only on the activation time and a time constant $\tau=20 \mathrm{~ms}$ as an assumed minimum refractory period. The definition and extraction of the activation time is described in detail in Section 4.2. Already during the detection of the activation times, they are only considered valid if there is more than $40 \mathrm{~ms}$ between successive activations. This translates to a time of at least $2 \cdot \tau$ between activations and this assumption is used during the tracking.

Given the time $t_{a}$ when an activation occurred, the method used here in practice defines the phase for the time span $t=\left[t_{a}-\tau, t_{a}+\tau\right]$ as:

$$
\varphi(t)=\pi \cdot\left(t-t_{a}\right)
$$

which gives $\varphi\left(t_{a}\right)=0$. If no activation exists for which $\left|t-t_{a}\right|<\tau$ applies the phase is chosen as $\varphi(t)=\pi$.

\subsubsection{Identification of Wavefronts and Phase Singularities}

Wavefronts can now be identified between areas that were activated no longer than the time $\tau$ ago and those that will be activated within a time $<\tau$ after the activated area - the activation is propagating. In this view, this is equivalent to a phase shift of $<\pi$ between the two neighbouring areas/pixels. PS are then naturally identified at the end points of each wavefront. Wavefronts ending at a boundary do not have a PS at this boundary. For the purpose of the algorithm, these are nevertheless stored as PS but are specifically marked. This process is sketched in Figure 4.4 for a linear reentry as indicated by the arrow. The red colours indicate the area that is activated next and the green line shows the wavefront. The figure includes an unexcited area (gray) and an area where activation is detected with a small delay $(t=5,8,10)$. A similar graph is also shown in Figure 4.5 for the experimental data. Panel (a) shows the time since the previous activation - corresponding to to the blue in the Figure 4.4 and Panel (b) gives the PS, the PS movement, the wavefronts, and the direction of 

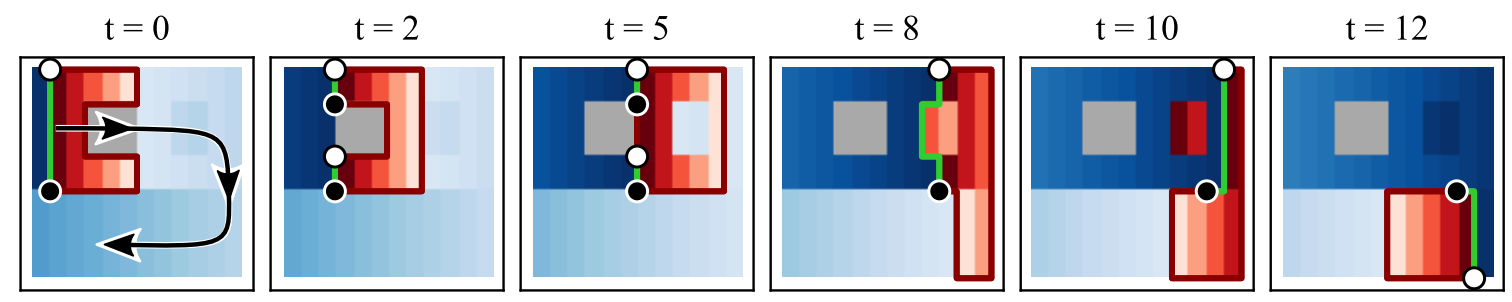

Fig. 4.4 (Figure and caption reproduced from the paper [85]) Sketch of the PS tracking. The plot shows a clockwise rotating spiral with the green line identifying the wave front. The spiral movement is indicated by the arrow in the first panel. Red colors indicate those pixels that will be activated within the next five frames with the darkest red indicating activation within the next timestep. Blue colors show those areas that were recently activated with lighter colors indicating a longer time since the last activation. PS are identified by black or white dots. The wavefront connects a PS pair, while their possible future track is identified by a red line. In this plot $\tau=5 . t=2$ and 5 show the behaviour at an inactive (gray) area, $t=8$ and 10 at an prematurely activated area and $t=12$ at the turning point.

movement. This direction of movement is indicated by the blue lines and, together with the green wavefronts, surrounds the area that will be activated next.

Technically, finding the wavefronts is achieved by employing a marching squares algorithm [90] to find the lines enclosing the area that will be activated within the time $\tau$. The marching squares algorithm used is a modified version from scikit-image [91], which places the wavefront always on the edges between pixels and ensures that two wavefronts ending on the same point are connected. This modification is necessary to simplify consistent tracking and identification. The tracking itself is based on the lines along the future activation (blue lines):

- If a PS is the closest to one with the same chirality existing in the previous frame - as defined along the direction of future travel (blue lines) - this is a movement event.

- If a PS did not move, a pair creation must have occurred. It is possible to extract which PS are associated in the creation event by inspecting the movements of direction (blue lines) from the previous frame.

With this approach some further information can be extracted and associated with each PS:

- The wavefront it belongs to, as well as the PS paired with it at the opposite side of the wavefront

- The direction of future movement, which is guaranteed to be along the area that will be activated next (blue lines in Fig. 4.5). Additionally, the next PS along this line can be found. If an annihilation occurs, the next PS along the direction of movement will be the PS annihilating together with the associated one.

- Whether the PS is a true PS or if it is on the boundary. 
a) activation detection

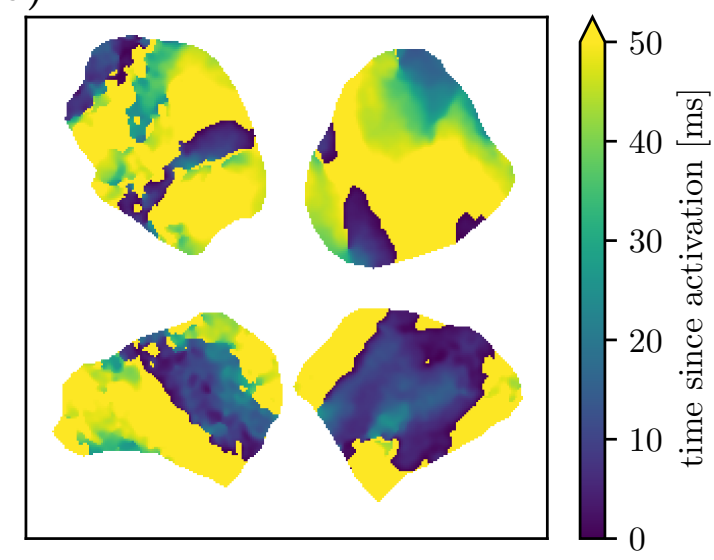

b) PS results

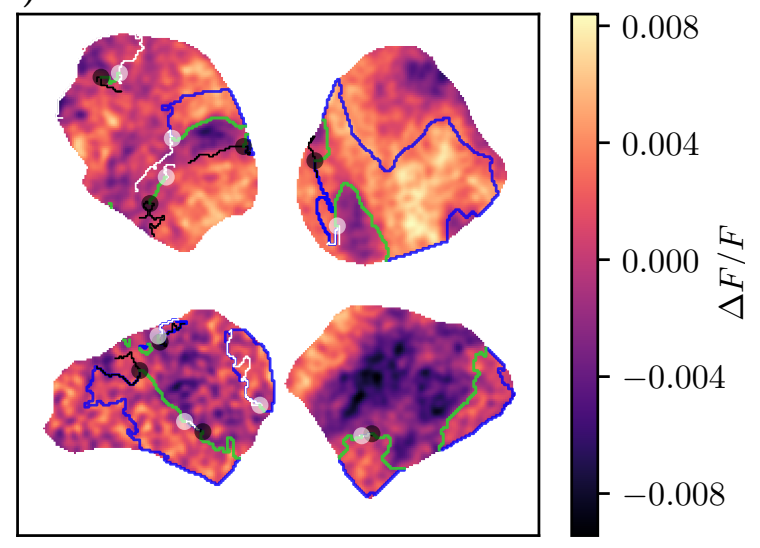

Fig. 4.5 a) Time since the last activation occurred. b) Smoothed fluorescence signal indicating PS (black and white circles) and wavefronts (green lines). The blue lines indicate the future movement direction of the PS and, together with the wavefronts, encircle the area activated within a time $\tau$ (within the next $20 \mathrm{~ms}$ ). (The figure reproduces the last panels of Figure 4.1 from the beginning of the chapter).

With these steps, it is thus possible to find and track all PS in the video. Further, it allows to store neighbourhood, creation, and annihilation information in a network or graph connecting all associated PS.

The strength of the approach described here is that no optimization or nearest neighbour search is necessary for the tracking. The reliable tracking is a direct byproduct of the given assumptions and inspection of the area that is activated next, i.e. the minimum refractory time and wave propagation speed.

\subsubsection{Filtering of Short Lived Phase Singularities}

Since noise such as misdetected pixels can easily create short lived PS, PS with a short life span are filtered in a second step. Here, this is achieved by employing the following greedy strategy:

- Set a minimum life span starting with one camera frame.

- Iterate through all frames:

1. Find all PS with life span shorter than currently allowed.

2. Find the PS with the shortest life span, if more than one such PS is found prefer the one closer to its neighbour in the direction of movement.

3. Attempt to remove the PS and the one it is associated with along the direction of movement.

- If this failed, attempt to remove the PS and the one it is associated with along the wavefront.

4. Scan whether the removal prolonged the life span of other PS. This occurs if a short lived pair is created along the movement path of an existing PS. 
After that pair is removed, the existing PS can move "through" it, while before it was annihilated and a new one created. ${ }^{8}$

- Iterate through all frames in reverse order:

1. Find the PS with the shortest life span, if more than one PS is found prefer the one associated with a shorter wavefront.

2. Attempt to remove the PS and the one it is associated with along the wavefront.

- If this failed, attempt to remove the PS and the one it associated with along the direction of movement.

3. Scan whether the removal prolonged the live span of other PS.

- Increase the minimum life span by one.

- If the minimum allowed life span reached its final value stop the iteration, otherwise start the whole process again.

In general a removal is only allowed if the PS were just created or are annihilated in the next frame. Further, at all times every PS must be paired with one of opposing charge both along the wavefront and its future path. Additionally, every PS is always paired with one of opposing charge for the creation and annihilation. Because of these constraints, in rare cases some short lived PS cannot be removed. Removing these would require more sophisticated strategies to ensure that no spatially inconsistent wave patterns would be associated with them.

The data shown in this thesis were filtered by removing all (or almost all) PS with a life span up to and including $30 \mathrm{~ms}$. The strategy itself iterates in a greedy fashion and appears to work well in general. It may be an interesting research topic to employ graph optimization algorithms or new approaches to optimize the removal strategy.

As a last step, phase singularities which only disconnect from the outside boundary for a short time, are also marked as boundary PS (and thus not true PS).

\subsubsection{Estimating Creation, Annihilation, Entering, and Leav- ing Rates}

For the data analysis in Chapter 6 it is necessary to classify each new PS as creation or entering, and each removed PS as an annihilation or leaving event. To do this classification the information of whether a PS is on the boundary and thus not considered a true PS is used. When a new PS appears it is counted as a creation if:

- it was not previously on the boundary

- the PS it was created with is not currently on the boundary,

otherwise it is counted as an entering event. The same approach is taken for a PS that disappears. It is counted as an annihilation if:

\footnotetext{
${ }^{8}$ This is alike the notion of compound rotors [92].
} 
- it does not continue to exist on the boundary

- the PS it annihilates with was not a boundary one,

and otherwise as a leaving event.

For each camera frame with the same NPS all of these events and how often the NPS value occurred are counted. From this it is possible to estimate the probability or rate of each of the events as a function of the current NPS. Since the probability of observing a creation, annihilation, entering, or leaving event is a Poisson event, the square root of the number of observed events is used as an error estimation for the fitting in 6 .

\subsubsection{Limitations of PS Identification and Tracking}

PS identification is inherently difficult for cardiac optical mapping data. This is largely due to the complex patterns and also the limitations of the optical mapping itself (compare Sec. 2.2.1) such as noise and motion artifacts. Often noise and regions of poor quality can create mostly short lived PS which are not real. However, even by eye it is often not easy to clearly identify the exact wave patterns.

Another limitation in this thesis is that the four cameras are analyzed separately and results are always given as the sum over all cameras. It would be a great improvement if a three dimensional surface reconstruction was used in the future. The separate analysis of the cameras means that meandering spirals can switch between two cameras. In some cases the same PS can also be visible from two cameras at the same time.

\subsection{Notes on Images and Plots}

To clarify possible confusion, here some notes on the plots and images shown in the thesis are given.

\section{Boxplots}

Boxplots are commonly employed to compare groups of data. In all cases boxplots are shown with $50 \%$ of the data points indicated by the box. The line inside the box indicates the median value. For the boxplots shown in this thesis the whiskers were always drawn to give the $9 \%$ to $91 \%$ range and all points outside are drawn as dots. Because of this, in some cases many "outliers" are indicated.

\section{Colourbars}

The image colourbars shown are sometimes limited to a central range. Limiting the colourbar inproves the contrast in the interesting regions. Whenever, the colourbar was limited this is indicated as an "arrow" at the corresponding end of the bar. 


\section{Chapter 5}

\section{Ischemia Characterization using Pinacidil}

The main datasets analyzed in this chapter and the following chapters were measured in a series using the drug Pinacidil. This experimental series was designed to change the dynamics of ventricular fibrillation to study variations in defibrillation energy observed in previous experiments. The drug Pinacidil was chosen for inducing changes similar to those occurring during ischemia. Here, the focus is on the characterization of the different states of the heart. While more experiments were performed during the course of my $\mathrm{PhD}$, these experiments provide a good basis for analysis. Further, some of the previous experiments were created with a focus on detailed ECG measurements which limited the video analysis that is the focus in this thesis.

The experimental series investigated here studied ten hearts and contains hundreds of episodes of ventricular fibrillation. This large dataset enables the characterization and further studies in the following chapters.

\subsection{Pinacidil as Ischemia Model}

In Section 1.6 ischemia - the reduced flow of blood - is described as a major change commonly occurring in hearts. This change can be intermittent, since blood flow may be impaired and then regained later on. For the patient ischemia is a side effect both during arrhythmia as well as a common disease on its own. This leads to questions:

1. What are the changes in characteristics of the dynamics such as activation times or phase singularities?

2. Do these changes have an effect on the defibrillation threshold, and can they explain observed fluctuations seen in the defibrillation success?

As the manipulation of parameters directly related to ischemia is difficult, the drug Pinacidil is used to achieve similar effect. Most importantly Pinacidil also leads to the shortening of the action potential. 
Thus, Pinacidil can be used to model a condition similar to ischemia in the heart, and, most central to this thesis, Pinacidil changes the dynamics of the tissue within a single heart during the experiment. This gives the opportunity to compare the properties of the turbulence within a single heart in different dynamic regimes.

While defibrillation study is always a major part of the experiment and a driving motivation behind our research, defibrillation energy and success statistics are only shown in Appendix A.2 (p. 144) for reference. Instead, a different approach will be used in Section 8.4 to discuss the effects of Pinacidil on how electrical shocks can control and affect the arrhythmias. Before presenting results based on novel analysis approaches, this chapter provides an overview and characterizes the changes observed with Pinacidil.

\subsubsection{Effects of Pinacidil}

The drug Pinacidil opens the ATP sensitive potassium channels $\left(\mathrm{K}_{\mathrm{ATP}}\right)$. In a clinical setting it is used to reduce blood pressure and thus not to directly related to the heart.

Under normal conditions, with a high ATP and low ADP concentration, the $\mathrm{K}_{\mathrm{ATP}}$ channels are predominantly closed. At low ATP and high ADP concentration they are predominantly open. Thus, when the heart becomes ischemic, the channels will open, which in turn causes the action potential to shorten. Pinacidil also causes these ATP sensitive potassium channels to open, and thus at a high enough concentration of the drug, an effect similar to that caused by a low ATP and high ADP concentration is expected. Pinacidil therefore models one of the symptoms of ischemia, although the strength of this effect is amplified by existing ischemia [93]. In experimental and computational studies Pinacidil has been found to shorten action potentials and increase the frequency of the arrhythmia [93-95]. The similar drug Cromakalim was found to have a comparable effect on the porcine heart and was found to stabilize spiral waves [96].

\subsection{Experimental Design}

The experiments were designed to increase the Pinacidil concentration in steps until a significant effect on the action potential was seen. While doing this, evaluation of the current state of the heart was done with multiple measurements. First activation time measurements were performed at the new Pinacidil concentration. Then fibrillation was induced and a long video recorded ( $15 \mathrm{~s}$ to $20 \mathrm{~s}$ ), although sometimes fibrillation could not be induced, especially early in the experiments. Additionally, at all times the heart state was observed during sinus rhythm and pacing with a fixed cycle length of $300 \mathrm{~ms}^{1}$ Using these measurements the shape of action potential was evaluated using the triangularity index (see Sec. 4.3.4). This was done already during the experiment to decide whether or not to increase the Pinacidil concentration further. After

\footnotetext{
${ }^{1}$ Except in the rare event that the sinus rhythm was faster.
} 


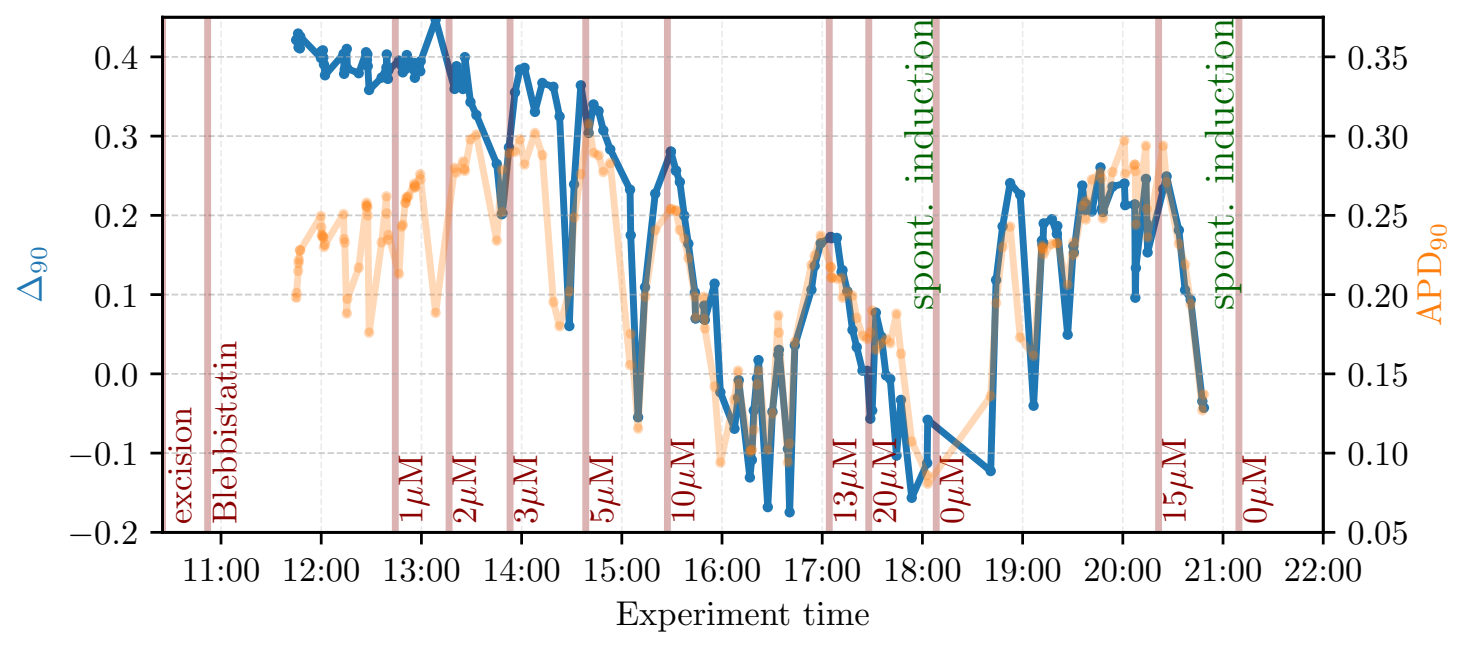

Fig. 5.1 Action potential triangularity index and action potential duration over time for the 2017-09-21 experiment. A smaller triangularity index corresponds to more pointy AP shape associated with ischemia. Changes in the Pinacidil concentration are indicated in red. "spont. induction" indicates times when arrhythmia started spontaneously and the Pinacidil concentration was subsequently reduced. Lines for the action potential and triangularity index are provided to guide the eye. Note that both the $\mathrm{APD}_{90}$ and the $\Delta_{90}$ are affected by arrhythmic episodes: After an arrhythmia both drop considerably and then recover within a short time. The values are calculated from averaged signals of one camera viewing the left ventricle (compare Sec. 4.3.3f).

the initial increase in the Pinacidil concentration many defibrillation trials and thus also VF measurements were performed as described in Section $2.6 \mathrm{f}$. When a sufficient number of defibrillation tests were performed, or if the heart became unstable during the measurement ${ }^{2}$, the perfusion was switched back to fresh Tyrode's solution without Pinacidil. This change in perfusion allowed the heart and action potentials to recover. The method of first testing Pinacidil was chosen to avoid changes over time confounding with the effect of Pinacidil.

Example traces of how the action potential changes at different times in the experiment are shown in Figure 5.2 and Figure 5.1 provides an overview of the extracted triangularity index and the APD over time in the experiment. In this experiment the concentration was slowly increased until the action potential was mostly triangular and with triangularity index of about 0.1. Since it was an early experiment, this concentration was studied for a time and then increased further. In later experiments the Pinacidil concentration was increased in larger steps in order to minimize the time required to find an optimal concentration. This is necessary since the time until Pinacidil takes full effect appeared to be around twenty minutes, meaning that multiple increments in the concentration would take several hours of measurement

\footnotetext{
${ }^{2}$ Meaning the spontaneous induction of VF not allowing a recovery time between VF episodes.
} 
Table 5.1 Overview over pinacidil experiments.

\begin{tabular}{cccc} 
experiment day & No. & age (months) & weight $(\mathrm{kg})$ \\
\hline $2017-09-21$ & 52336 & 10 & 3.4 \\
$2017-09-28$ & E26703 & 15 & 4.5 \\
$2017-10-05$ & E26712 & 15 & 3.2 \\
$2017-10-12$ & 52279 & 10 & 3.9 \\
$2017-10-17$ & 52333 & 10 & 3.5 \\
$2017-11-02$ & F25998 & 4.5 & 3.4 \\
$2017-11-16$ & F25990 & 5 & 3.7 \\
$2017-11-23$ & F25955 & 5 & 3.6 \\
$2017-11-30$ & F25950 & 5 & 3.6 \\
$2018-03-28$ & F37064 & 7 & 3.9
\end{tabular}

time.

\subsubsection{List of Experiments}

In total ten experiments were analyzed as listed in Table 5.1. All animals were female New Zealand white rabbits. For the general experimental procedures compare also Chapter 2. More information about who performed the experiments as well as timelines of some of the experimental parameters can be found in the Appendix A (p. $137 \mathrm{ff})$.

\subsection{General Analysis Notes}

The majority of the work here is performed studying the arrhythmic episodes recorded during this experimental series. These episodes in general are divided into two groups:

1. $3 \mathrm{~s}$ or $5 \mathrm{~s}$ long $\mathrm{VF}$ episodes recorded before each defibrillation attempt.

2. Occasional $15 \mathrm{~s}$ to $30 \mathrm{~s}$ long recordings of VF episodes.

Because a short VF episode does not necessarily provide enough data for a clear statistical analysis, the short episodes are grouped together if they are within a similar time span in the experiment. Additionally, each of these time span groups are manually checked to ensure that the dynamics did not fluctuate too strongly. Because of this approach, most of the later analysis of arrhythmia thus consists of 31 groups. These 31 groups are composed of 16 long VF videos recorded at a specific time and 15 groups of short VF episodes which lie within time spans ranging between 30 minutes and three hours. 


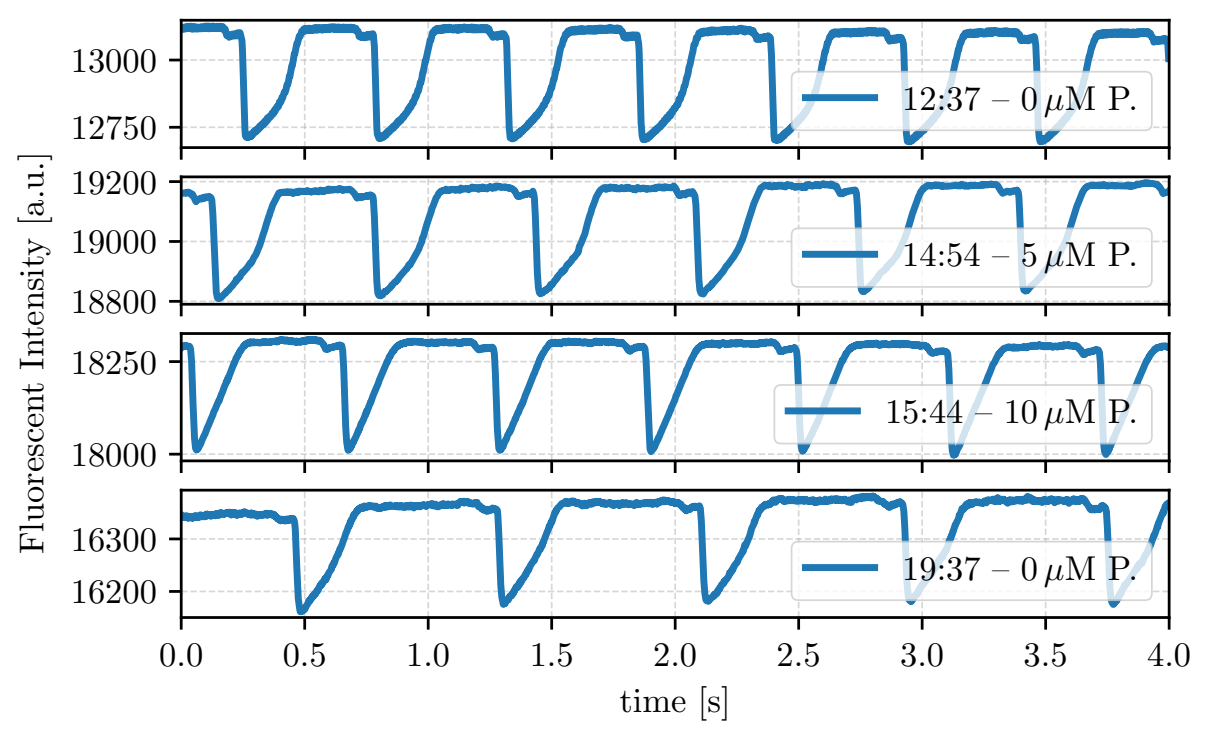

Fig. 5.2 PseudoECG of a single camera looking onto the left ventricle during normal sinus rhythm at four different times in the experiment (average fluorescent signal of the whole camera). The experiment day is 2017-09-21. From top to bottom the concentration of Pinacidil increases from $0 \mu \mathrm{M}$ over $5 \mu \mathrm{M}$ to $10 \mu \mathrm{M}$ and is then reduced again to $0 \mu \mathrm{M}$. Especially the action potentials at $10 \mu \mathrm{M}$ have less of a plateau but the form partially recovers when going back to $0 \mu \mathrm{M}$ Pinacidil. For $5 \mu \mathrm{M}$ the action potential is longer, most likely due to a slower rhythm.

\subsection{Results}

In this section the changes caused by Pinacidil are characterized. The same data is analyzed under different aspects also in the following chapters. This chapter continues with the following parts:

- The actional potential shape change caused by Pinacidil will be presented.

- The results of the activation maps performed during the experiments is given. This result will be necessary for interpreting the observations in Chapter 8 .

- Results from the analysis of VF with and without Pinacidil are given and compared.

- Note that a brief overview of the defibrillation testing performed can be found in Appendix A.2 (p. 144).

\subsubsection{Control of APD as a Marker for Pinacidil Effect}

In Figure 5.2 the action potential as recorded from the average fluorescent signal from one camera looking onto the left ventricle is shown. During the course of the experiment performed on the 2017-09-21, the Pinacidil concentration was increased 
regularly and the first strong effect on the action potential shape was seen at $10 \mu \mathrm{M}$ (third panel). This was later reversed by replacing the Pinacidil containing Tyrode's solution with fresh solution causing a recovery, albeit not a full one, as shown in the last panel.

Observing the change of the action potential shape, as well as its control through Pinacidil was thus successful in this experiment. However, the effect of a certain concentration on the heart was unfortunately not very reproducible. This made the precise control more difficult than anticipated, since the same Pinacidil concentration had a varying effect on different hearts.

Another apparent problem was that at too high concentrations, the hearts would show spontaneously arising, self-induced VF. This is problematic, since a resting time of five minutes between measurements of VF is desirable to achieve long lasting stable experimental conditions. Without any resting periods image quality and health of the heart will deteriorate.

However, in all except one experiment the desired change in the action potential form could be achieved, and many experiments had stable enough conditions for long measurements of defibrillation shocks and thus VF episodes. Even after discarding many videos with poor quality the remaining data provides the opportunity to analyze the arrhythmic dynamics with and without Pinacidil in several hundred episodes that add up to a total of about one hour of VF. Further, in many of the hearts both the dynamics with and without Pinacidil can be compared directly. The measurement and estimation of the triangularity index during the experiment provided us with a tool to quantify the effect of the drug and guide the experiment.

\subsection{Activation Time Analysis Results}

For each level of Pinacidil concentration activation maps were made after at least 15 minutes passed to allow the new concentration to take effect. These activation maps allow to assess changes on the recruitment of wave emitting sites and provide an important base line for the results in Section 8.4. As the voltage increases excitation waves are initiated at more and more places during the electrical shock, which in turn leads to a faster activation of the observed epicardial surface. For the theoretical background and motivation of activation time analysis compare also Section 3.1. Section 4.3.1 details how the activation times are calculated from the experimental data. The main result of the analysis here is the characterization of the activation time $\tau$ which denotes the time required for a pulse of a certain strength to activate $95 \%$ of the ventricular surface.

Figure 5.3 shows an example of an activation map for the 2017-10-05 experiment at 15:53 with Pinacidil and increasing shock voltage and the shock duration "pulse width". A typical activation pattern is visible with the first activation occurring close to the apex or within the septum reaching the epicard only with a significant delay of about $30 \mathrm{~ms}^{3}{ }^{3}$ At high voltages, the first areas activated are on the left ventricle

\footnotetext{
${ }^{3}$ The tiny area activated at the top is an artifact due to a reflection or proximity to the atrium.
} 

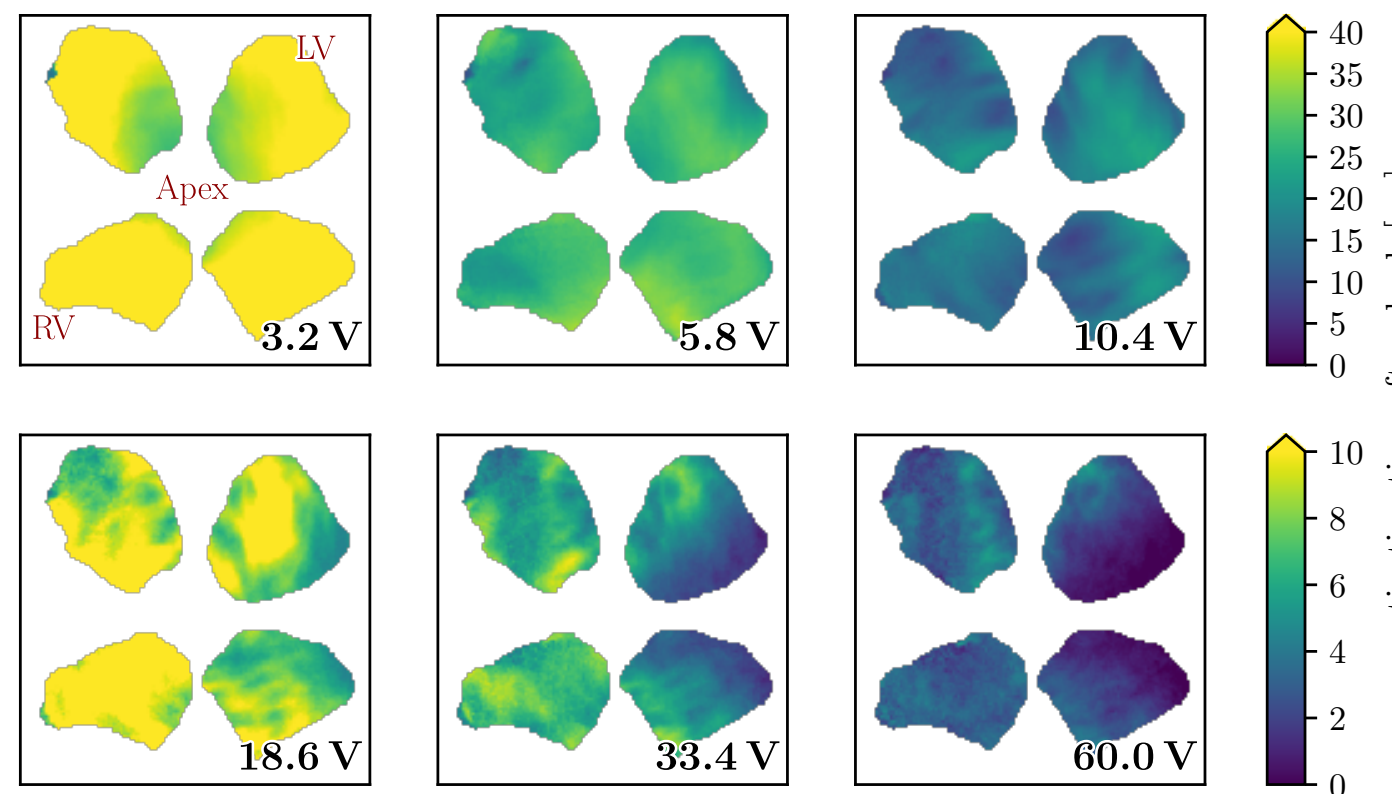

Fig. 5.3 Activation time maps recorded in the experiment 2017-10-05 at 15:53 with a pulse width of $5 \mathrm{~ms}$ and at a Pinacidil concentration of $7 \mu \mathrm{M}$. The panels show the behaviour with increasing shock voltage. The colours indicate how many millisecond after the shock a specific area on the hearts surface was activated. The top and bottom row use a different scaling, since the activations times in the top row are much longer. For the heart geometry and field of view, compare also Figure 4.1 (p. 34).

a)

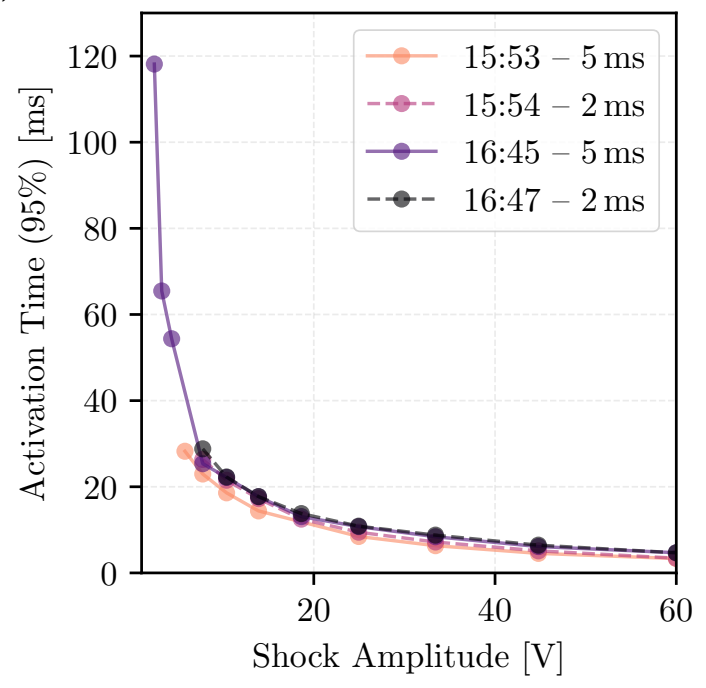

b)

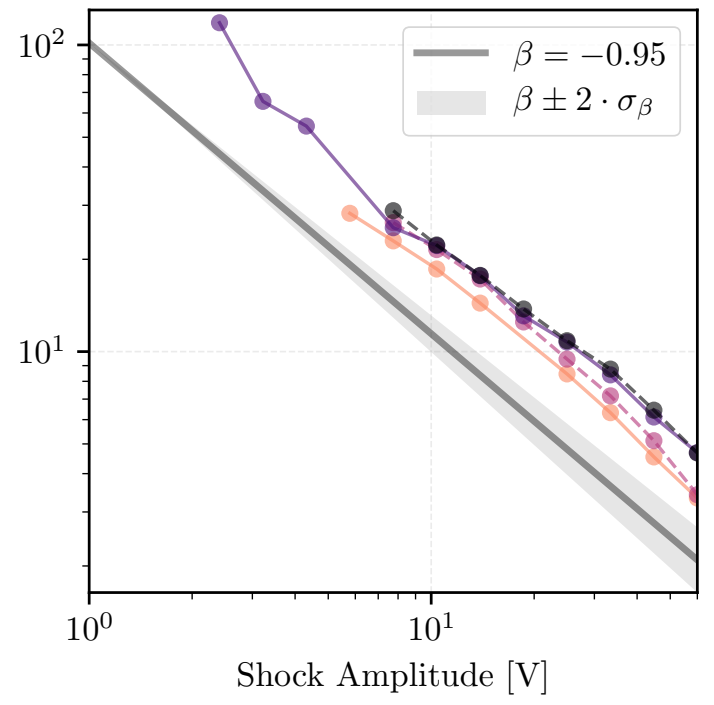

Fig. 5.4 Activation times (2017-10-05 experiment). The activation times indicate how long it took until $95 \%$ of the area was activated. a) Activation time plotted against the shock voltage and $\mathbf{b}$ ) the same but plotted on a double logarithmic axis. The slope and error of the slope fit to the activation times are indicated. 
(right side in the plot) with wave emission originating close to the left ventricular wall (compare Figure 4.1 and for the activation patterns Figure 8.1 on page 109).

Figure 5.4 shows the time it took for the $5 \mathrm{~ms}$ pulse width shocks of a specific voltage to activate $95 \%$ of the area ${ }^{4}$. This is given at four different times and with two Pinacidil concentrations during the experiment 2017-10-05. Panel (a) gives the activation times, while Panel (b) plots the times on a double logarithmic scale with a fitted power law slope indicated. A slight shift to shorter activation times is visible for the later measurements (15:53 and 16:45), however, inspecting further such plots manually no systematic shift in $\tau$ as a function of the voltage for the Pinacidil concentration itself is apparent.

For a specific shock voltage and pulse width, the measurements of $\tau$ typically fluctuate by a value of $\lesssim 0.2 \mathrm{~ms}$. Because of these very stable results the errors are not shown and were not used during the fitting procedure. Above approximately $7 \mathrm{~V}$ ( $10 \mathrm{~V}$ for $2 \mathrm{~ms}$ pulse width) the activation times follow a power law in most experiments. This is theoretically expected (compare Sec. 3.1) and thus the power law $\tau(E)=\tau(1) \cdot E^{-\beta}$, where $E$ is the electric field strength given by the voltage, is fitted to the double logarithmic application of the data using linear least squares.

A full table of all fit results can be found in the Appendix A.3 (p. $146 \mathrm{ff}$.). Instead of giving $\tau(1)$ the tables and plots list the activation time at $20 \mathrm{~V}$, so $\tau(20 \mathrm{~V})$ as a more intuitive measure to compare the shift of the activation time curves.

\subsubsection{Activation Time Overview for the Different Experiments}

Table 5.2 lists the activation time fit parameters and their standard deviations as calculated over the multiple activation time measurements done for each day as well as the mean and standard deviations calculated over all experiments. Some experiments - especially 2017-09-21 - do not show a perfect exponential behaviour, which explains the partially high values. However, most of the values for the slope $\beta$ agree fairly well. Further, there is no significant difference between the two pulse widths, giving a value of about $\beta=-0.79$ for both measurements.

\subsubsection{Dependency on the Pinacidil Level}

As already mentioned above, individual experiments do not seem to show a change of the activation time curves due to Pinacidil. This indicates that a direct effect of the Pinacidil on the shocks itself is either small or does not exist. This will be important in Chapter 8 since it shows that the ability to recruit certain wave emitting sites is unaffected by Pinacidil. Figure 5.5 shows a boxplot comparing the power law fit parameters of slope and offset for all experiments where a comparison between Pinacidil and no Pinacidil was possible. This shows that no apparent differences in

It is too small to have an effect on the final result but could be masked for the analysis.

${ }^{4} 95 \%$ is chosen for robustness to small errors. Varying the value does change the result slightly but not significantly. 
Table 5.2 Table summarizing the measured pulse width (p.w.) for each day giving the slope $\beta$, the activation time at $10 \mathrm{~V}$, and the voltage at which the activation time is $20 \mathrm{~ms}$ (as extracted from the fit parameters) as well as their standard deviations (when missing, less than three curves were recorded). The unweighted mean and standard deviation over all days is given in the "all" row.

\begin{tabular}{lllll}
\hline & & $\beta$ & $\tau_{10 \mathrm{~V}}$ & $\mathrm{~V}_{20 \mathrm{~ms}}$ \\
day & p.w. $[\mathrm{ms}]$ & & & \\
\hline $2017-09-21$ & 2 & $-0.621 \pm 0.099$ & $32.0 \pm 3.6$ & $21.3 \pm 2.5$ \\
& 5 & $-0.707 \pm 0.069$ & $31.2 \pm 2.2$ & $18.9 \pm 2.2$ \\
$2017-09-28$ & 2 & $-0.724 \pm 0.110$ & $20.4 \pm 2.0$ & $10.2 \pm 1.2$ \\
& 5 & $-0.718 \pm 0.086$ & $18.7 \pm 1.8$ & $9.0 \pm 1.3$ \\
$2017-10-05$ & 2 & $-0.994 \pm 0.090$ & $22.0 \pm 2.0$ & $11.0 \pm 1.0$ \\
& 5 & $-0.935 \pm 0.086$ & $19.3 \pm 1.9$ & $9.6 \pm 1.1$ \\
$2017-10-12$ & 2 & $-0.753 \pm 0.043$ & $23.7 \pm 2.1$ & $12.5 \pm 1.5$ \\
& 5 & $-0.749 \pm 0.072$ & $20.6 \pm 1.4$ & $10.4 \pm 0.9$ \\
$2017-10-17$ & 2 & $-0.808 \pm 0.127$ & $29.9 \pm 2.4$ & $16.5 \pm 1.5$ \\
& 5 & $-0.803 \pm 0.101$ & $26.5 \pm 1.5$ & $14.3 \pm 1.2$ \\
$2017-11-02$ & 2 & $-0.795 \pm 0.006$ & $23.8 \pm 1.8$ & $12.4 \pm 1.2$ \\
& 5 & $-0.801 \pm 0.019$ & $22.2 \pm 0.1$ & $11.4 \pm 0.1$ \\
$2017-11-16$ & 2 & $-0.818 \pm 0.078$ & $29.5 \pm 2.4$ & $16.0 \pm 0.9$ \\
& 5 & $-0.781 \pm 0.053$ & $32.5 \pm 3.1$ & $18.6 \pm 2.1$ \\
$2017-11-23$ & 2 & $-0.766 \pm 0.116$ & $35.4 \pm 2.4$ & $21.2 \pm 1.6$ \\
& 5 & $-0.803 \pm 0.096$ & $37.9 \pm 5.0$ & $22.1 \pm 2.5$ \\
$2017-11-30$ & 2 & -0.867 & 24.2 & 12.5 \\
& 5 & $-0.846 \pm 0.029$ & $21.4 \pm 1.3$ & $10.9 \pm 0.8$ \\
\hline all & 2 & $-0.794 \pm 0.102$ & $26.8 \pm 5.1$ & $14.8 \pm 4.2$ \\
& 5 & $-0.794 \pm 0.069$ & $25.6 \pm 6.8$ & $13.9 \pm 4.8$ \\
\hline
\end{tabular}

the activation times are caused by Pinacidil. Additionally, systematic differences in the propagation velocity should be small. This is visible since a faster propagation velocity would shift the activation time curves to smaller values on the logarithmic scale (compare eq. 3.7).

\subsection{Results Characterizing the Arrhythmia}

As mentioned at the beginning of the chapter, most of this study is limited to the study of episodes of ventricular fibrillation (VF) from manually chosen time spans during the experiment. These time spans may include multiple episodes that are of sufficient quality for the video analysis and appear to have stable experimental conditions. Most of the analyzed episodes are $3 \mathrm{~s}$ or $5 \mathrm{~s}$ segments of VF recorded just before a defibrillation shock was given. The recording length differs slightly between some of the experiments. Since also long videos with a length of $15 \mathrm{~s}$ to $30 \mathrm{~s}$ were 

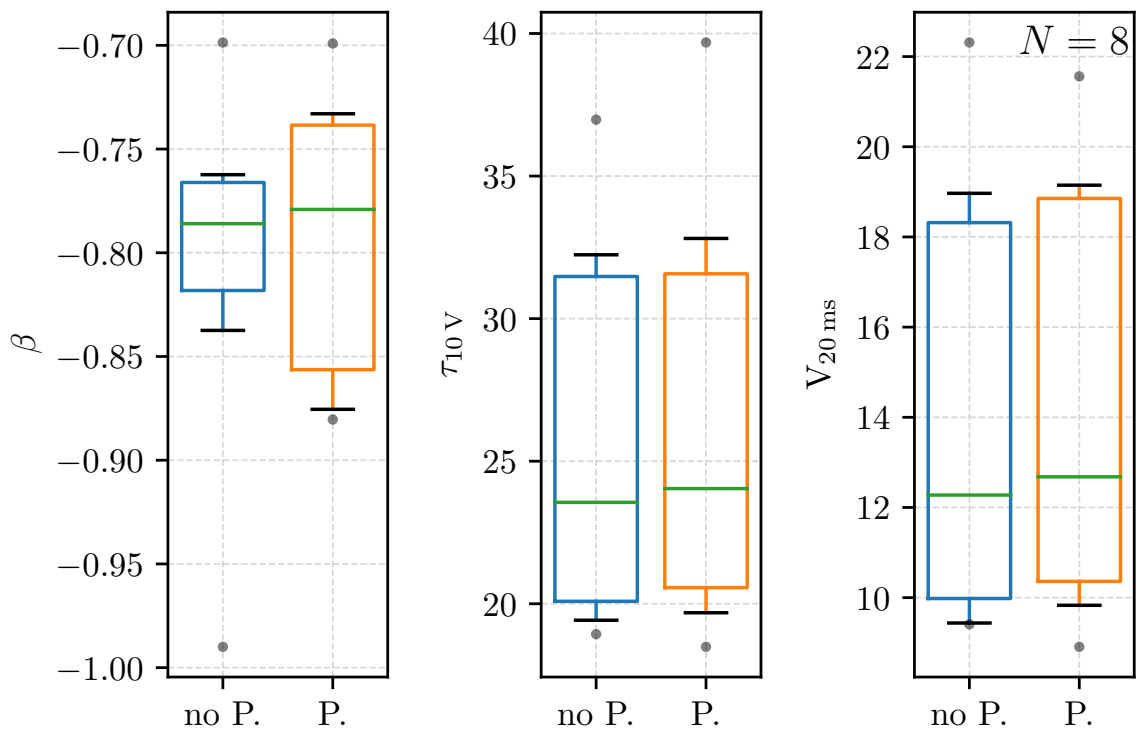

Fig. 5.5 Boxplot giving the fit parameters of the activation time curves as measured without and with Pinacidil. The comparison is made for activation times with a pulse width of $5 \mathrm{~ms}$ from a total of eight experiments. No significant change due to Pinacidil can be found both in slope or offset of the activation maps.

made, these are also analyzed below. However, when such a long video is within a time span containing short VF episode it is not included in the analysis to ensure a better comparability of the various time span data.

Table 5.3 provides an overview of the parameters calculated from this data. Like almost all data analysis in this thesis, these results are calculated based on all four cameras. This lists the average number of phase singularities (NPS), the average over the dominant cycle length (CL), the average estimated $\mathrm{APD}_{50}$ as well as general information. For all of these values the exact definitions and analysis methods are detailed in Section 4.3. The detected NPS usually ranges between 10 and 20 in these experiments. The CL usually takes values below $100 \mathrm{~ms}$ indicating a typical frequency of the arrhythmia of about $10 \mathrm{~Hz}$.

Figure 5.6 shows a direct comparison for those times where the parameters with and without Pinacidil can be directly compared within the same experiment (day). Some of the trends that are expected with ischemia and the changes caused by Pinacidil are visible:

- The NPS increases with the addition of Pinacidil.

- The CL decreases or, equivalently, the frequency of the arrhythmia increases.

- The APD also clearly decreases.

While the trends with respect to the change caused by Pinacidil are clear, the variability between the hearts is, however, also strong in these experiments. 
Table 5.3 Overview over parameters derived from and describing different VF episodes. These are grouped by the experiment day, time range and Pinacidil (P.) concentration. Only videos of manually confirmed quality were used. This includes both recorded individual movies as well as VF recorded before a defibrillation attempt. The total number of videos analyzed for each of the events, the VF episodes (ep.) and the total duration (dur.) are also given. While the NPS and its standard deviation are calculated over all combined episodes, the $\mathrm{CL}$ and $\mathrm{APD}_{50}$ means and standard deviations over their individual episode value.

\begin{tabular}{|c|c|c|c|c|c|c|c|}
\hline day & time & P. $[\mu \mathrm{M}]$ & NPS & $\mathrm{CL}[\mathrm{ms}]$ & $\mathrm{APD}_{50}[\mathrm{~ms}]$ & ep. & dur. $[\mathrm{s}]$ \\
\hline \multirow[t]{5}{*}{ 2017-09-21 } & $11: 00-12: 30$ & 0 & $15.1 \pm 5.7$ & $90.1 \pm 6.9$ & $53.6 \pm 6.5$ & 21 & 100 \\
\hline & $11: 50$ & 0 & $13.0 \pm 5.1$ & 94.5 & 58.2 & 1 & 29.8 \\
\hline & $13: 11$ & 1 & $15.0 \pm 5.6$ & 87.5 & 58.9 & 1 & 19.8 \\
\hline & $13: 43$ & 2 & $14.6 \pm 5.4$ & 76.5 & 49.5 & 1 & 29.8 \\
\hline & $19: 30-20: 20$ & 0 & $16.0 \pm 8.2$ & $94.7 \pm 9.3$ & $53.1 \pm 9.1$ & 20 & 95.2 \\
\hline \multirow[t]{3}{*}{$2017-09-28$} & $12: 08$ & 0 & $10.6 \pm 4.8$ & 95.5 & 45.6 & 1 & 29.8 \\
\hline & $14: 30-15: 10$ & 0 & $21.6 \pm 6.9$ & $81.5 \pm 3.3$ & $35.9 \pm 0.7$ & 3 & 14.3 \\
\hline & $14: 45$ & 0 & $16.3 \pm 5.6$ & 91.5 & 46.4 & 1 & 29.8 \\
\hline \multirow[t]{4}{*}{ 2017-10-05 } & 13:00-16:00 & 7 & $20.6 \pm 6.5$ & $66.4 \pm 4.0$ & $28.4 \pm 2.1$ & 83 & 395 \\
\hline & $13: 13$ & 7 & $21.8 \pm 5.9$ & 67.5 & 29.5 & 1 & 29.8 \\
\hline & $16: 40-18: 50$ & 0 & $14.2 \pm 7.1$ & $77.4 \pm 5.8$ & $34.8 \pm 5.2$ & 49 & 233 \\
\hline & $17: 02$ & 0 & $9.6 \pm 4.5$ & 78.5 & 37.7 & 1 & 29.8 \\
\hline 2017-10-12 & 16:00-18:10 & 0 & $14.2 \pm 4.8$ & $81.2 \pm 4.5$ & $40.1 \pm 3.7$ & 56 & 185 \\
\hline \multirow[t]{3}{*}{$2017-10-17$} & $14: 08$ & 22 & $14.2 \pm 5.5$ & 78.5 & 46.2 & 1 & 19.8 \\
\hline & $16: 00-19: 15$ & 63 & $17.0 \pm 6.8$ & $70.9 \pm 12.6$ & $34.3 \pm 3.7$ & 90 & 428 \\
\hline & $20: 15-22: 10$ & 0 & $16.9 \pm 9.4$ & $81.8 \pm 8.2$ & $37.5 \pm 6.5$ & 14 & 66.6 \\
\hline 2017-11-02 & $18: 05$ & 0 & $20.4 \pm 5.9$ & 86.5 & 38.9 & 1 & 9.76 \\
\hline \multirow[t]{4}{*}{ 2017-11-16 } & 12:00-12:38 & 16 & $17.5 \pm 5.6$ & $105.6 \pm 9.6$ & $52.4 \pm 5.8$ & 8 & 38.1 \\
\hline & $13: 10-14: 20$ & 24 & $23.5 \pm 6.8$ & $83.6 \pm 5.9$ & $37.2 \pm 3.7$ & 30 & 143 \\
\hline & $14: 20-15: 00$ & 24 & $28.5 \pm 8.0$ & $80.2 \pm 5.7$ & $35.4 \pm 2.7$ & 20 & 95.2 \\
\hline & $16: 05-18: 50$ & 0 & $17.1 \pm 6.1$ & $125.1 \pm 13.2$ & $66.0 \pm 8.1$ & 74 & 352 \\
\hline \multirow[t]{5}{*}{$2017-11-23$} & 18:00-19:50 & 15 & $7.9 \pm 4.6$ & $94.6 \pm 15.0$ & $44.8 \pm 8.7$ & 56 & 157 \\
\hline & $15: 28$ & 0 & $6.0 \pm 4.6$ & 135.5 & 84.8 & 1 & 19.8 \\
\hline & $17: 42$ & 15 & $8.5 \pm 4.2$ & 94.5 & 46.7 & 1 & 19.8 \\
\hline & 20:08 & 15 & $8.1 \pm 4.2$ & 113.5 & 51.2 & 1 & 14.8 \\
\hline & $20: 23$ & 15 & $7.8 \pm 3.8$ & 109.5 & 46.4 & 1 & 14.8 \\
\hline \multirow[t]{4}{*}{$2017-11-30$} & $11: 36$ & 0 & $9.9 \pm 4.8$ & 113.5 & 60.8 & 1 & 29.8 \\
\hline & $12: 30-14: 45$ & 10 & $22.3 \pm 7.2$ & $85.1 \pm 4.6$ & $33.4 \pm 2.4$ & 52 & 248 \\
\hline & $15: 40$ & 0 & $19.6 \pm 6.3$ & 82.5 & 33.6 & 1 & 29.8 \\
\hline & $16: 00-17: 50$ & 0 & $14.3 \pm 6.8$ & $94.8 \pm 7.7$ & $40.2 \pm 4.0$ & 53 & 158 \\
\hline $2018-03-28$ & $12: 35$ & 0 & $13.0 \pm 5.2$ & 107.5 & 67.8 & 1 & 29.8 \\
\hline
\end{tabular}


a)

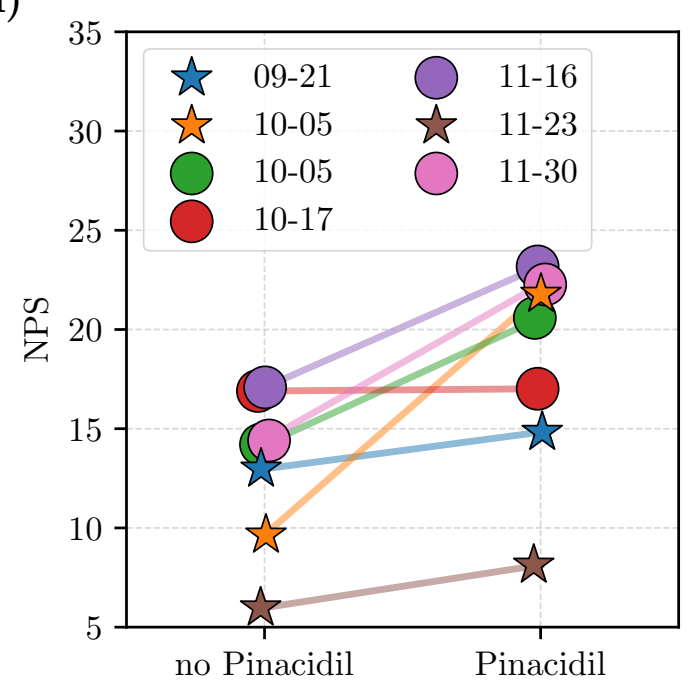

b)

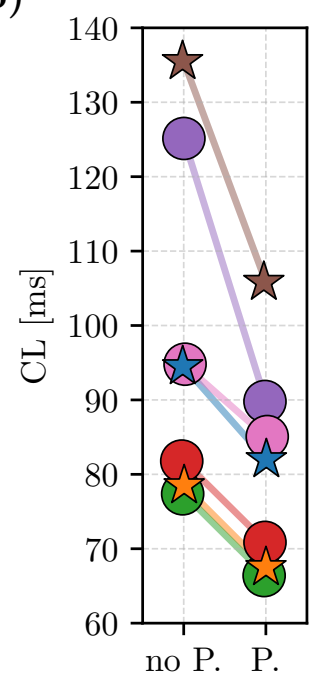

c)

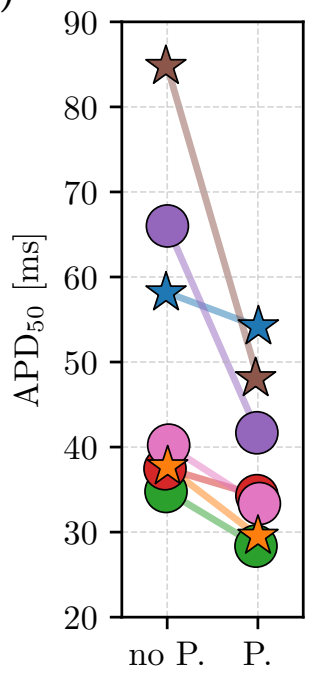

Fig. 5.6 Comparison of the changes in parameters describing the VF caused by Pinacidil. Each circle corresponds to the average of all time spans with multiple episodes $(3 \mathrm{~s}$ or $5 \mathrm{~s}$ long) on the given day with or without Pinacidil. Each star identifies the parameters derived from a single long episodes. Only experiments where a direct comparison was possible are shown. a) The average NPS, as an average over the individual episodes, $\mathbf{b}$ ) the estimated dominant $\mathrm{CL}$ and $\mathbf{c}$ ) the approximated $\mathrm{APD}_{50}$.

\subsection{Summary}

In this chapter the changes caused by Pinacidil as a model for ischemia were presented and the experimental procedure with respect to testing Pinacidil detailed.

The activation time results show that a power law can explain the activation time as a function of shock voltage in the intact rabbit ventricles. The value for the power law slope of $\beta=0.794 \pm 0.1$ can be compared to the results for canine ventricular wedge preparations with $\beta=-0.73 \pm 0.17$ and pig ventricular wedge preparations with $\beta \approx-0.98$ as presented previously in $[4,20]$. Additionally, no effect of Pinacidil on the activation times was found. This means that the the wave emitting sites during a far field shock are most likely unchanged by the drug, a fact that will be important in Section 8.4.

Considering previously reported changes in other hearts [94-96], the changes to the arrhythmia caused by Pinacidil in the rabbit heart are not surprising. However, they provide a comparison for Chapter 6 and characterize the effects of Pinacidil in these experiments. Further, they confirm that Pinacidil indeed is capable to model many of the changes observed during ischemia. 


\section{Chapter 6}

\section{Analysis of the PS Markov Model and its Parameters}

In Section 3.2, the theoretical foundation for describing the number of phase singularities on the heart using a Markov model, was derived and motivated. While a stochastic model is not capable of capturing the deterministic chaos which is used to model the heart in simulations, such a model and its descriptive parameters of entering, leaving, annihilation and creation rates of PS can characterize the arrhythmia and may provide insights into the dynamical properties of the arrhythmia.

Sections 6.1 and 6.2 present results directly related to the Markov model. The interpretation of these results is then be discussed in in Section 6.3. Furthermore, the temporal fluctuations of the NPS are discussed with respect to the autocorrelation function of the NPS in Section 6.4 and from the perspective of nonstationary behaviour in the following section.

\subsection{NPS Rates of Change}

For the previously described arrhythmic episodes and groups of episodes (time spans, see Sec. 5.3), it is possible to estimate the four rates by which the number of phase singularities (NPS) changes as a function of the current NPS as detailed in Section 4.4. Briefly, as described in the theory Section 3.2, the four rates are:

- entering: The rate at which single PS enter the field of view seen by the cameras.

- leaving: The rate at which single PS leave the field of view seen by the cameras.

- creation: Twice the rate at which pairs of PS are created, so that each PS counts towards the rate individually.

- annihilation: Twice the rate at which pairs of PS collide and annihilate with each other, so that each PS counts towards the rate individually.

Additionally, entering and creation rates as well as leaving and annihilation rates may be combined into: 


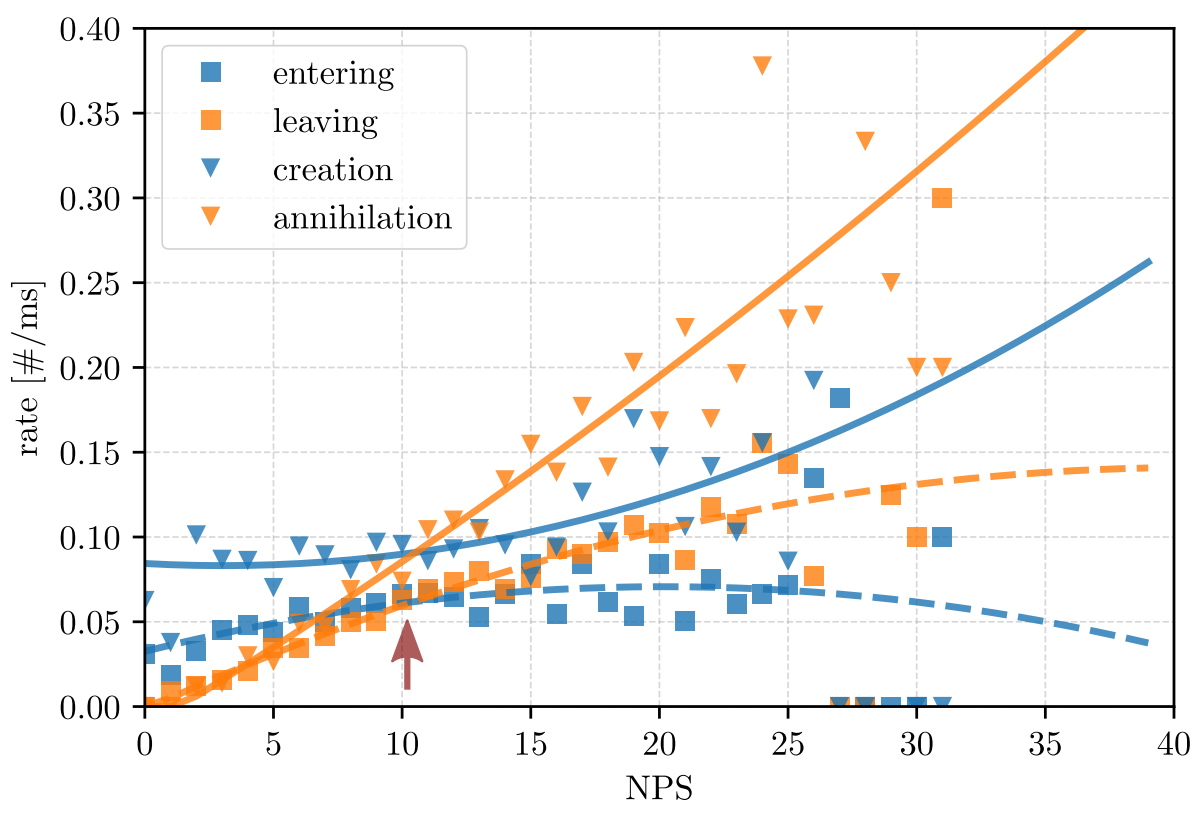

Fig. 6.1 Rates of change of the NPS for the episode recorded 2017-09-28 at 12:08 (30 s of VF). The symbols give experimentally measured rates for how fast the NPS changes for each observed NPS. The rates of change are split into the four basic mechanisms by which the NPS changes: entering, leaving, creation, and annihilation. The lines indicate the result of a quadratic polynomial fit to each of the four basic rates (see also eq. 3.9). The arrow marks the intersection points of creation and annihilation rates, and entering and approximately gives the average NPS. At higher NPS values leaving and annihilation dominate while at lower NPS values creation and entering are dominant. The figure, as all analysis in this chapter, is calculated using all four cameras.

- total creation: Sum of entering and creation rates.

- total destruction: Sum of leaving and annihilation rates.

This is used briefly in Section 6.2 to highlight that pair creation and pair annihilation are necessary to capturing the observed behaviour.

An example of this analysis can be seen in Figure 6.1 where the estimated rates for the $30 \mathrm{~s}$ episode recorded on 2017-09-28 at 12:08 is given. This analysis, as all analysis in this chapter, is made by analyzing all four cameras. In this example a few characteristics which are shared by most analyzed episodes can be observed:

- Creation and entering rates have a predominantly constant contribution, often with a small additional increase with the NPS.

- Annihilation and leaving rates increase with the NPS, in this example, the increase appears largely linear.

- For intuition: The intersections of creation/annihilation and entering/leaving rates approximately give the mean NPS as marked by the arrow. 
When interpreting the rates, it may be important to distinguish between short and long measurements of $\mathrm{VF}$, because the rates may change with time. When analyzing time spans that include many short VF episodes, it is possible to estimate the rates over all of them or individually. While the Markov model approach can be successfully used for both, rates calculated from multiple episodes and long videos may have to be interpreted differently.

Here, both the analysis of each individual short episodes as well as the combined modelling for many VF episodes within a time span, are used side by side:

- Sections 6.1.1 and 6.1.2 discuss possible information that may be inferred from the rates. To avoid effects of pooling data, the rates for these are calculated for individual recordings of VF. Thus, the models:

$$
\begin{aligned}
r_{\text {creation }}(\mathrm{NPS}) & =\text { const }+m \cdot \mathrm{NPS} \\
r_{\text {annihilation }}(\mathrm{NPS}) & =m \cdot \mathrm{NPS}+b \cdot \mathrm{NPS}^{2}
\end{aligned}
$$

will be fitted. Fewer parameters lead to easier interpretation here. Also, since the VF episodes are short, fitting of more complex models is less reliable.

- Section 6.2 discusses the probability distribution more closely focusing on the long episodes or pooling all VF episodes within each time span. Since more data is available here, more parameters can be estimated and lead to a better approximation of the probability distribution. Thus, generally the model:

$$
r_{\text {all rates }}(\mathrm{NPS})=\mathrm{const}+m \cdot \mathrm{NPS}+b \cdot \mathrm{NPS}^{2}
$$

will be used for all rates for simplicity.

Section 6.5 will discuss and interpret the variation in the rates that occurs over time.

\subsubsection{Results from the Pair Creation Rate}

One of the most interesting properties of the creation rate is the observation of its linear trend as a function of the NPS. Depending on the dynamical properties, the creation rate may for example be constant or increase with a higher NPS, which in turn may help to understand what mechanisms are driving the turbulent state. To analyze the change of the rate with increasing NPS, here a linear model is fitted to the experimental rates. Such a linear model captures the main contributions to the rate and allows to quantify an existing trend from the fit parameters.

As noted before, to avoid issues due to possible non-stationary behaviour, here the short $3 \mathrm{~s}$ or $5 \mathrm{~s}$ VF episodes are analyzed individually. For each of the time spans statistics are given over all VF episodes within either in the form of boxplots or mean values.

This analysis leads to Figure 6.2 summarizing the observed linear contribution to the creation rate for each time span. The figure shows the parameters derived 

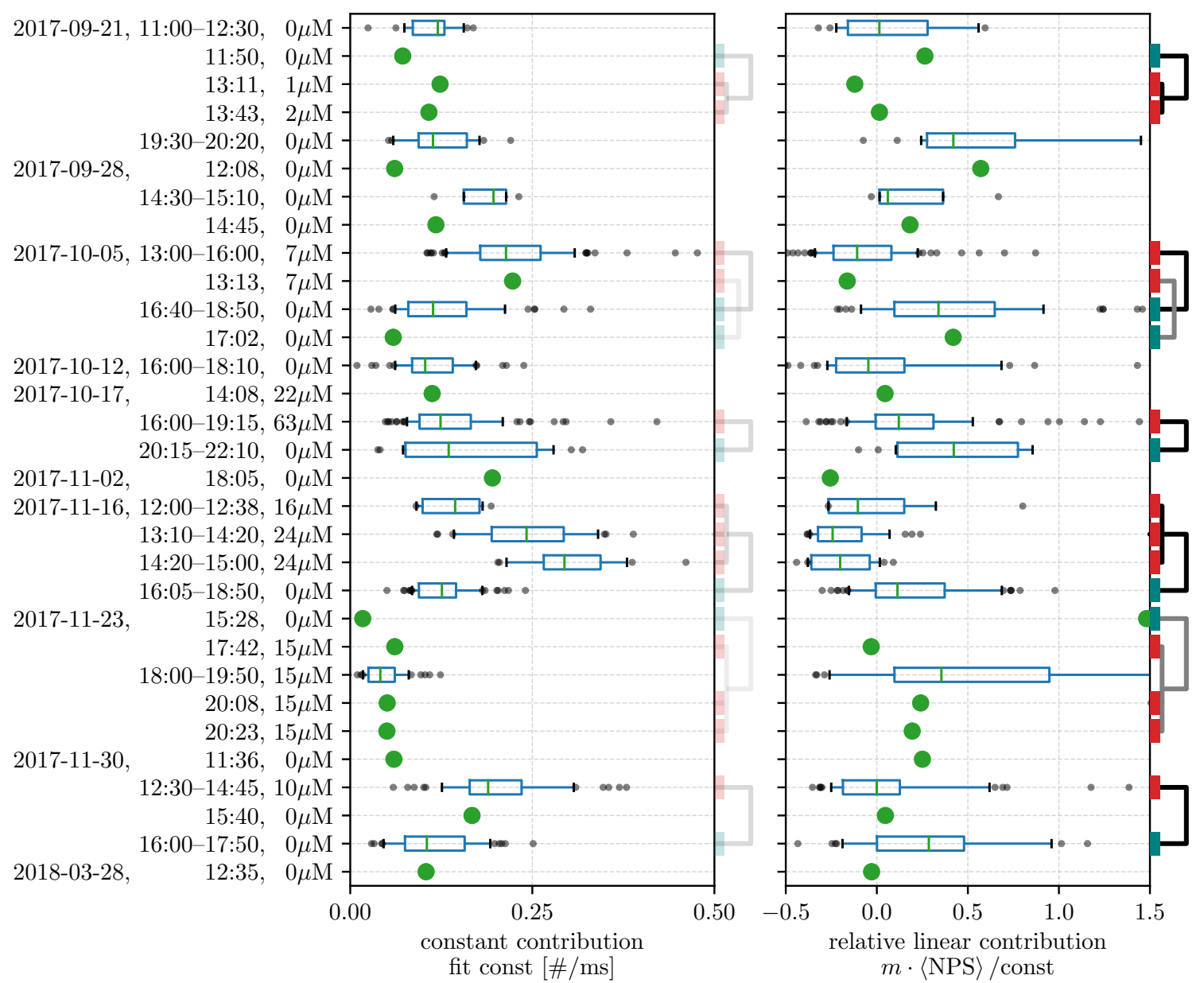

Fig. 6.2 Comparison of the importance of the constant and linear contribution to the creation rate for all time spans. Points indicate single long videos (15 s to $30 \mathrm{~s}$ ) and boxplots multiple short $(3 \mathrm{~s}$ or $5 \mathrm{~s})$ VF episodes within the time span. The $y$-axis lists the experiment day, time span, and Pinacidil concentration. The first panel shows the constant fit parameter of $r_{\text {creation }}(\mathrm{NPS})=$ const $+m \cdot \mathrm{NPS}$ and the second the linear contribution normalized as a relative contribution compared to the constant component. Brackets next to the axes indicate which times are used to compare the dynamics with and without Pinacidil. Their mean values are shown in 6.3 for a direct comparison. 
a)

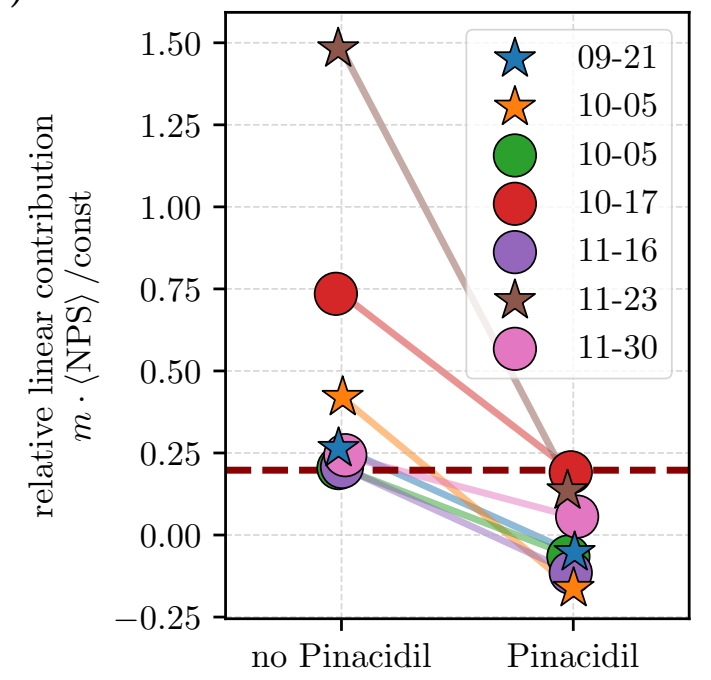

b)

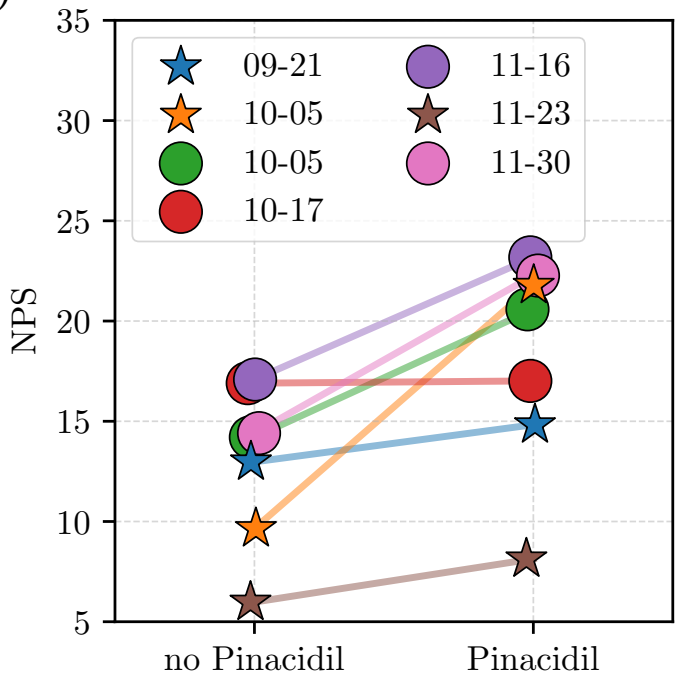

Fig. 6.3 a) Comparison of the relative linear contribution to the creation rate with and without Pinacidil. The plot compares the mean values of the brackets indicated in Figure 6.2 calculated over all VF episodes within the time span. Stars are calculated from single long VF episodes while circles correspond to the mean of time spans containing $3 \mathrm{~s}$ or $5 \mathrm{~s}$ episodes. The red line indicates a separating line between the two groups. b) The same comparison done for the average NPS reproduced from Figure 5.6 for comparison.

from the fit $r_{\text {creation }}(\mathrm{NPS})=$ const $+m \cdot$ NPS. Specifically, $m \cdot\langle$ NPS $\rangle /$ const is given to quantify how large the relative linear contribution is with respect to the constant term. The values for this relative linear contribution are typically around zero and well below 0.5. For example the two time spans 2017-09-21 at 19:30 and 2017-10-17 at 20:15 without Pinacidil have a relative linear contribution on the order of, or above, 0.25 .

The plot also indicates groups of time spans or episodes which may be compared to find differences due to Pinacidil. The mean values for each of the brackets are also shown in Figure 6.3 for a direct comparison. It is apparent that in all cases, six experiments and seven groups, the linear contribution is higher with Pinacidil. While the fact that both groups separate is certainly by chance, the difference is clear. Considering that these time spans and thus box plots include many independent events, random fluctuation cannot explain the differences in the rate contribution for the individual experiments. Independent of the changes due to Pinacidil, a change can, however, also be found for the two time spans starting at 11:00 and 19:30 2017-09-21. For these times, the later one has a strong linear contribution while the first one has not. This may indicate that over the course of this long experiment the dynamics of the arrhythmia changed as well. A further exception to the trend of lower linear contribution with Pinacidil is 2017-11-23 at 18:00-19:50. Here, and also in the single VF videos of the same day, the linear contribution appears relatively high considering that these are recorded with Pinacidil. This may be partially explained by the low 
NPS and the long cycle lengths observed, which may indicate that Pinacidil affected the heart to a smaller extent in this experiment.

Thus, while the variation in Figure 6.2 is quite high it confirms the intuition gained when examining examples of the observed creation rates: Positive linear contribution is, in these experiments, typically not the dominant contribution. However, it does show a clear contribution for many events. This contribution appears especially strong for the healthy conditions and seems to be reduced by Pinacidil.

The most important result of this section is the apparent sensitivity of the new parameter to Pinacidil. A more detailed discussion and interpretation is given in Section 6.3.

\subsubsection{Results from the Pair Annihilation Rate and Pair Statistics}

Similar to the above considerations about the creation rate, the annihilation rate warrants a closer look with respect to the contributions of the linear and quadratic terms as a function of the NPS. However, it is difficult to draw a clear picture from this. To give a notion of the two contributions, Figure A.12 (p. 152) shows the average contribution both for the linear and quadratic term of the model rate $r_{\text {annihilation }}(\mathrm{NPS})=m \cdot \mathrm{NPS}+a^{2} \cdot \mathrm{NPS}$. These coefficients quantify the contribution of the terms to the total rate.

Except for some long episodes, no clear dominant term can be seen when comparing the linear with the quadratic contribution. There also appears to be little systematic trend for which of the two is larger. This indicates that neither a purely quadratic nor a purely linear model is likely able to fully capture the annihilation rate during VF. A meaningful quantitative comparison between the linear and quadratic terms should be taken with a grain of salt. On the one hand, it seems clear that a purely quadratic model cannot sufficiently describe the data for most events. On the other hand, a purely linear model will inevitably miss an additional larger than linear contribution for many NPS. Additionally when comparing episodes of different Pinacidil levels no clear trend is seen in this data.

\section{Creation and Annihilation Pair Statistics}

As an alternative way to make a statement about how strongly pairs of PS tend to interact, it is possible to build a graph of PS creations and annihilations. From this graph, the fraction of phase singularities that annihilate together with the same PS that they were created with can be derived. This means that the creation and annihilation occurs between the same two PS. For the 16 long video data segments analyzed before, this fraction is $0.50 \pm 0.07$ and thus high compared to random collisions. ${ }^{1}$ In

\footnotetext{
${ }^{1}$ To calculate this statistics, wavefronts ending at the edge of the heart were considered to end in a PS that lies on the outer boundary. While mathematically not a PS, this ensures that PS are always created and annihilated in pairs.
} 


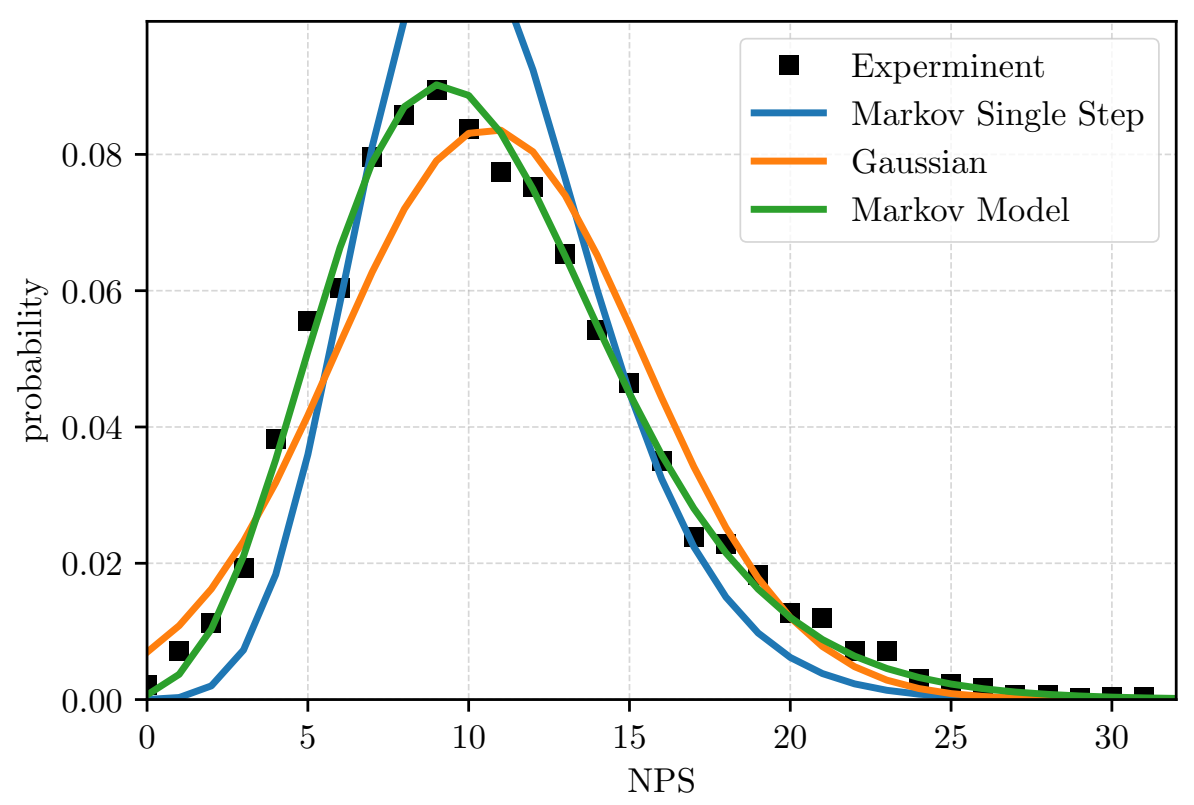

Fig. 6.4 Probability for observing a certain NPS for the same episode shown in Figure 6.1, consisting of a $30 \mathrm{~s}$ video of VF without Pinacidil. Additionally to the experimentally observed distribution (black squares), different models are shown. The Markov models distribution is derived from the curves fitted to the rates in Figure 6.1. Markov single step denotes a Markov model which does not include pair creation and annihilation, but otherwise reproduces the same total rates. The Gaussian model is a fit of the normal distribution to the data.

other words, half of the observed PS appear to be closely associated with a paired PS for their entire livespan. This PS is the one they were created with as well as the one they annihilate with.

\subsection{Results for the Probability Distribution of the NPS}

As detailed in Section 3.2.1 the NPS rates of change can be used to create a Markov model. This Markov model can predict the probability distribution of the NPS. To achieve this, the rates are fitted as introduced previously. Then, the detailed balance of the Markov model is solved to find the model's stationary probability distribution $\mathcal{P}(\mathrm{NPS})$ (compare Sec. 3.2.1). For comparison the NPS distribution is also estimated using:

1. A Gaussian model, derived from the mean and standard deviation of the experimental data.

2. A single step Markov model, which assumes that only events affecting a single PS exist and no pair creations and annihilations occur. Such a model thus views 


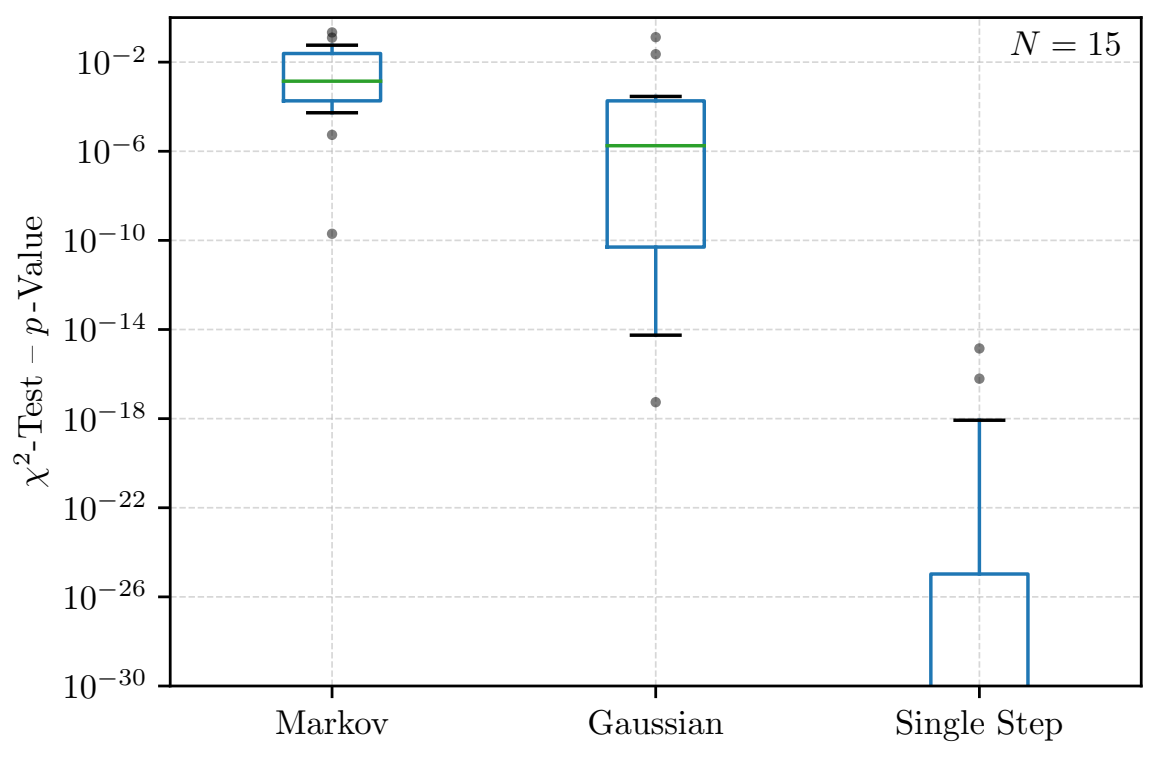

Fig. 6.5 $\chi^{2}$ test comparing the hypothesis that the PS distribution can be described using the different models. The boxplot summarizes the median of 100 repetitions over all 15 time spans with multiple short VF episodes. Each repetition uses half of the episodes to estimate the model, and the other half for the $\chi^{2}$-test. Higher values of the $\chi^{2}$ test indicate that the given model matches the experimental results better. They thus correspond to a better fit for example in Figure 6.4. 
a pair creation as two individual events.

An example for the results of this analysis for all three models can be found in Figure 6.4. It shows the three models as well as the experimentally observed distribution of the NPS for the same thirty second VF episode given in Figure 6.1.

To compare the Markov model's stationary distribution with the other alternative descriptions for the NPS distribution, this is tested in more depth for all time spans composed of many individual episodes. One approach to compare how well the models describe the experimentally observed distribution is the use of a $\chi^{2}$ goodness of fit test. Three problems arise when computing the test statistics:

1. Samples should be uncorrelated, but the NPS are correlated especially on the time scale of about one CL (compare Sec. 6.4).

2. The $\chi^{2}$ test is not reliable when bins contain very few events.

3 . The $\chi^{2}$ test requires knowledge of the degrees of freedoms of the model fitted to the data.

To reduce issues due to the first point, the NPS time series used for the test is subsampled at $100 \mathrm{~ms}$ intervals removing strong correlations ${ }^{2}$.

The second issue can be solved by combining multiple NPS probabilities/bins with very few data points into a single probability bin including adjacent NPS values with a higher number of events. Here this is achieved by combining multiple NPS levels until each contains at least five events. Since few events occur for either large or small NPS values, this combining of bins is done starting from the largest NPS bin going down, and then starting from zero NPS going up.

The third issue is avoided here by using only part of the data for the model estimation step and the rest of the data for the $\chi^{2}$ test. ${ }^{3}$ Thus, the rates are estimated using only half of the VF episodes within a time span (rounded up) and the test is then performed on the remaining half. Using such a training (model estimation) and validation ( $\chi^{2}$ test) strategy ensures that model estimation uses a separate data set. For the test this effectively means that there are no additional degrees of freedom contributed by the model parameters. This procedure is repeated 100 times with different splits of the data and the median over all 100 test results is reported.

The result of this procedure is summarized in Figure 6.5 shown as a boxplot over all 15 time spans. The higher value of the $\chi^{2}$-test for the Markov model shows that it performs better than for example a Gaussian one. While the $p$-Value for the probability of the Markov model matching the observed distribution is not very high, given enough data this is expected even for small model deviations.

To summarize the $\chi^{2}$-test results, the Markov model works well when looking at an average over multiple episodes and this appears to be true even for unseen data. ${ }^{4}$

\footnotetext{
${ }^{2}$ Although this should mostly affect the absolute values and these should be interpreted with care in any case.

${ }^{3}$ Such as training and validation/testing sets in machine learning, see for example [97].

${ }^{4}$ Although not necessarily for predicting an individual episode.
} 


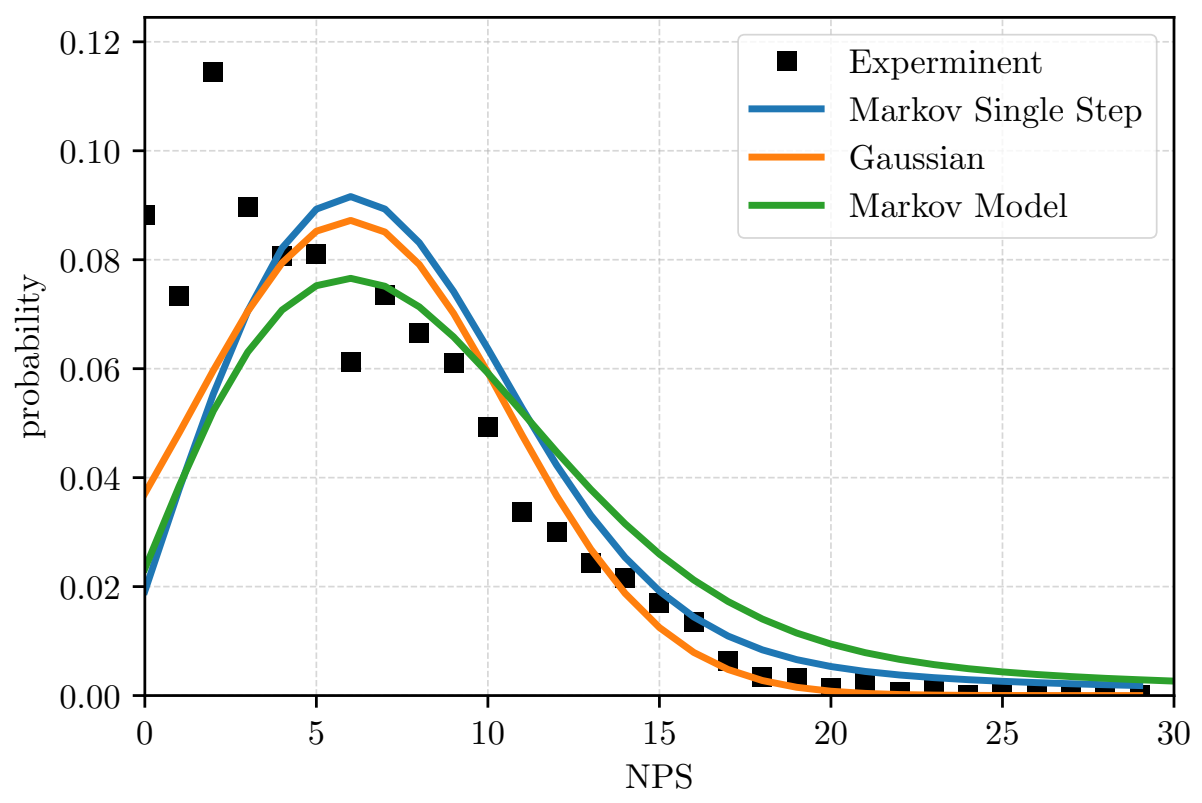

Fig. 6.6 Probability of observing a certain NPS for the episode in the experiment 2017-11-23 at 15:28. This video of a VF is $20 \mathrm{~s}$ long and no Pinacidil was used. From all 31 Markov models, it is the only exception showing a visually significant derivation of the Markov model from the experimental observation. Lines indicate the probability distributions of the different models and black squares the experimental data, as also shown in Figure 6.1 for a different episode.

Manual inspection of the NPS distributions from all time spans and VF episodes shows a visually good fit in all but one event. This exception is the VF episode 2017-11-23 at 15:28 and shown in Figure 6.6. Two overlapping reasons may explain the strong deviation:

- When the NPS drops too low on average, the model may not perform well due to hidden dynamics, for example within the septum, having a large influence. One may hypothesize that a hidden Markov model with additional unseen states may capture this to some degree. ${ }^{5}$

- This video visually shows repetitive patterns that quickly activate most of the heart akin to a VT. This could indicate that, more than in most events, the dynamics are driven by unseen dynamics not captured by the Markov model. Above, this was interpreted as a general shortcoming of the model when few PS are present. However, it could also be interpreted as the existence of a dominating part of the dynamics that is unseen and cannot be captured by

\footnotetext{
${ }^{5}$ A simple extension, by allowing negative NPS, indeed shows a large improvement. Such an extension may be reasonable even in other cases where the results do not change much since the probability to reach no NPS is low. However, the choice of the hidden states and their emission probabilities in the hidden Markov model add too much complexity to be discussed or analyzed in depth here. For a formal definition of hidden Markov models see for example [98, Ch. 3.2]
} 
a)

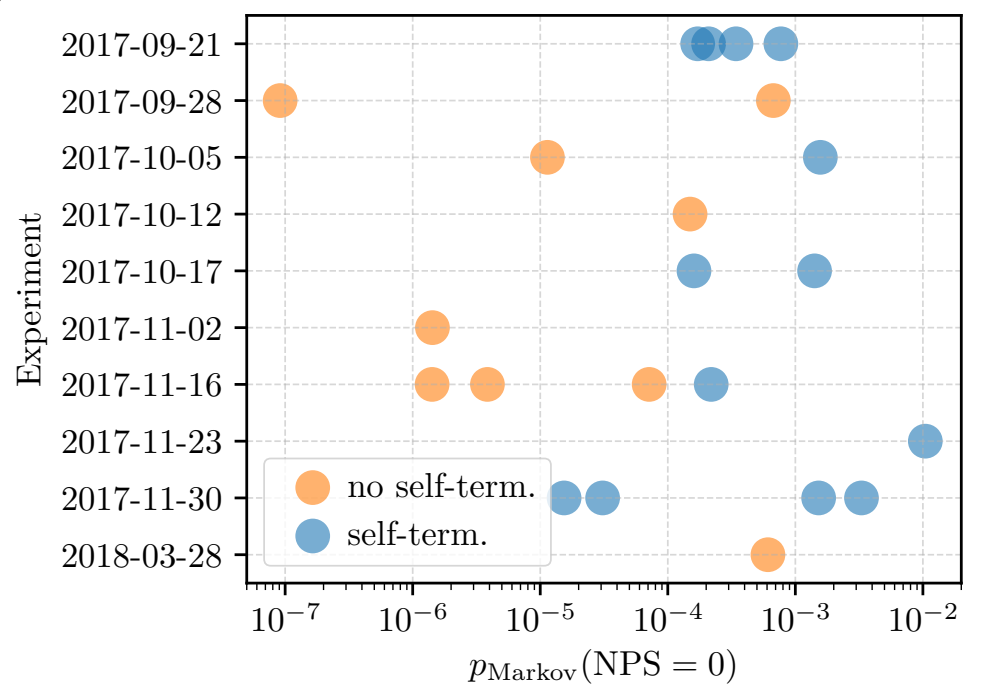

b)

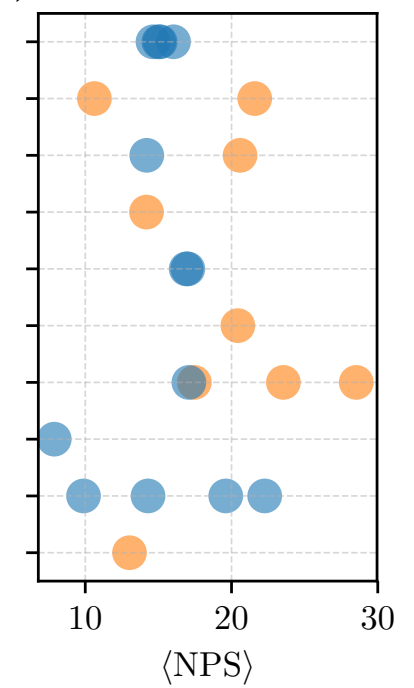

Fig. 6.7 For each of the experiment on the $y$-Axis each dot represents on time span with the colour indicating whether or not self-termination was observed. a) gives the probability of finding no PS in the Markov model on the $x$-Axis, while b) shows the average NPS. Both (a) and (b) give similar results with the average NPS being anti-correlated with the probability of no phase singularities $p_{\text {Markov }}(\mathrm{NPS}=0)$.

the stochastic Markov model. One possibility for this would for example be a relatively stable dominating spiral wave. In cardiac literature such a dominating rotor is called a "mother rotor" and its importance to sustaining the arrhythmia is contested. This is discussed for example in [2] and references therein. If this interpretation would be substantiated for example by simulations, it would suggest that in most cases a dominating mother rotor does not exist in the rabbit heart.

In general, the Markov model works very well to describe the probability distribution of the NPS for the analyzed time spans and videos. This is a behaviour shared with other pattern forming systems as well as the simulations mentioned in the theory Section 3.2. It further provides the basis for the following section where the knowledge of the probability distribution is used.

\subsubsection{Relation to Self Termination}

All data analyzed here was recorded from fully developed fibrillation that did not stop during the recording time. However, in some of these experiments self-termination, the spontaneous end of fibrillation, is observed. For example it happens that after inducing a VF, it stops within a short time ${ }^{6}$. Alternatively, a VF may stop even after a long time of fibrillation.

\footnotetext{
${ }^{6}$ If it stops too fast the induction would be considered unsuccessful itself.
} 
For the previously analyzed time spans it is now possible to fit the rates to find the Markov model and its stationary probability distribution. Since termination is characterized by the fact that no spirals remain, a necessary condition for it is that no PS is observed. Thus, the probability of finding no PS in the Markov model $p(\mathrm{NPS}=0)$ may be an indicator for whether or not self-termination was indeed observed. This is similar to the approach taken by Sugimura et al. for simulations [76].

Furthermore, it is possible to analyze all experiments and determine whether selfterminations occurred at or around a given time span. Here, this was done using two sources of information:

1. Often it was noted during the experiment when self-termination occurred.

2. When induction events were repeated within a short time, although the first one was noted as a successful induction, a self-termination has most likely occurred between the two.

While these records do not provide a quantification of how commonly self-termination occurred, they do provide an approximate categorization into whether or not it was observed at all.

The result of this analysis is given in Figure 6.7 where blue dots show episodes with observed self-termination and orange ones identify those without.

The correlation between $p(\mathrm{NPS}=0)$ and the average NPS is very high so that both provide similar information. However, the plot indicates that self-terminations may indeed be more likely when there are fewer PS observed and $p(\mathrm{NPS}=0)$ is large. The results shown in Figure 6.7 are based on a fairly small set of data and depend more on the heart than on whether or not Pinacidil was given. Further, the identification of whether or not self-termination occurred may be prone to errors. Nevertheless, the results motivate that such an approach to understand self-termination may not be limited to simulations and could be applied successfully to experimental observations.

\subsection{Interpretation of Markov Model Results}

The results shown in the previous sections can be interpreted with respect to the theoretical and numerical findings mentioned in the introductory Sections 3.2.2f. There are three parts to this.

First, the rate of creation is expected to rise with the NPS in three dimensional systems. This is shown by Davidsen and colleagues to occur with negative filament tension [72] or more generally as a surface effect [74]. Figure 6.2 quantifies the linear and the constant contribution to the pair creation rate. When looking at individual VF episodes, the pair creation rates typically do not show a dominating, but often a clear, increase with the NPS. This suggests that the third dimension in many cases, while certainly an important feature of the heart, may not play a dominating mechanistic part in the chaotic dynamics. The linear contribution may, to some degree, quantify whether the negative filament tension mechanism contributes to the turbulence, since 
a linear increase would be expected with a strong influence from the negative filament tension. A comparison with comprehensive numerical studies on realistic geometries could provide a better interpretation of this result. In the healthy rabbit heart, wave sources appearing on the epicardium are observed and from simulations it has been suggested that they are linked to negative filament tension $[12,99]$. The linear contribution parameter appears to agree with this observation and may help to quantify it.

In the experiments, the addition of Pinacidil appeared to removed the linear contribution from the creation rate. This suggests that negative filament tension may be less important with Pinacidil. In contrast, from a theoretical standpoint, naively, Pinacidil may be expected to cause the opposite, because a lower excitability increases the negative filament tension in models [12]. However, more factors, such as the increased excitable gap (compare Sec. 8.4), may be involved in the phenomenon.

With a better understanding based on simulations, the creation rate could help to give insights into the mechanisms driving the arrhythmia, such as the importance of negative filament tension. Considering that the rate appears to be affected by Pinacidil this can also add a new and useful way to characterize the dynamics.

Second, the rate of pair annihilation gives some information about the interaction of phase singularities. As noted in [78] the rate of pair annihilation should increase quadratically with the NPS if PS are interacting weakly. This is because both the NPS itself as well as the probability of a collision increase linearly. However, from Figure A.12, it is not clear that the quadratic contribution is dominant in a model that includes both linear and quadratic terms. This suggests that phase singularities in the heart are not weakly interacting. One reason for this is that PS commonly annihilate together with the PS they are created with and those pairs are most likely closely associated for their full live span. Using direct statistics, this was shown to occur for about half of the phase singularities.

Therefore, direct analysis and, to a lesser extend, comparing the quadratic and linear contributions to the annihilation rate both indicate that PS are commonly strongly interacting. Comparing the conditions with and without Pinacidil, there appear no significant changes in these values associated with the annihilation. This suggests that, in the rabbit heart, Pinacidil has a larger effect on the creation dynamics than the annihilation dynamics.

Third, chaotic excitable media, such as the heart, have the interesting property that self-terminations can occur spontaneously after a random time has passed. This time is known to scale exponentially with the domain size [77]. For estimating it, Markov model inspired approximations have been suggested to work well in simulation systems to deduce the probability of self-termination from the statistical properties of the NPS [76]. Figure 6.7 suggests that a similar argument may also be possible for the experimental system of the fibrillating heart. The probability of finding zero PS in the Markov model thus may allow to assess the chance of such self-terminations to occur even when self-terminations (or a drop to zero phase singularities) are not 
observed. In the future, such an approach should be revised by employing more advanced techniques available for Markov models: The time until the zero "absorbing" state is reached could in principle be computed directly in such a framework.

Additionally, interesting results could possibly be achieved by comparing these results with, or applying this technique to the atrium. Two main points may prove interesting. First, the atrium, which is better approximated by two dimensions, may show a different trend in the pair creation rate. Further, atrial fibrillation, which is a common disease and can in principle be studied in the patient itself, may be an interesting target for detailed analysis using these methods. For example, having a marker for how likely self-termination is, could guide the doctor when deciding whether or not a specific therapy is promising for the inhibition of atrial fibrillation.

These findings may also help in the validation of more realistic cardiac models, and in turn such simulations may provide new insights into the interpretation. Simulations should aim to reproduce and explain properties shown to exist in experimental data. New insights into the dynamical properties of the heart and how it changes due to drugs, may further guide the development of new therapies.

\subsection{Autocorrelation Function of the NPS}

A property of the NPS time series that has received little attention so far is its temporal autocorrelation function (ACF). This can be calculated directly from the experimental observation. Additionally, it can also be derived for the Markov model. This analysis also provides an estimate for the necessary delay until the NPS values can be assumed to be uncorrelated, as was necessary for the tests in Section 6.2.

Calculating the autocorrelation as a function of the time delay $\Delta t$ for both the experimental data and the derived Markov model gives Figure 6.8. In this particular event, the ACF drops relatively slowly compared to the one derived from the stochastic Markov process. One large difference between the true ACF and the one derived from the Markov model is that the former has an oscillatory component in addition to the approximately exponential decay over time. ${ }^{7}$ To separate the exponential decay time and find the period of the oscillatory component, here, an exponentially decaying cosine function added to an exponential term is fit to the experimental ACF by minimizing the squared error. The functional form used is ${ }^{8}$ :

$$
\mathrm{ACF}_{\text {fit }}(\Delta t)=\alpha_{1} \cdot e^{-\frac{\Delta t}{\tau_{1}}}+\left(1-\alpha_{1}\right) \cdot e^{-\frac{\Delta t}{\tau_{2}}} \cdot \cos \left(\frac{2 \pi \cdot \Delta t}{\text { period }}\right)
$$

\footnotetext{
${ }^{7}$ Such an oscillatory term is also present in simulations such as in the Bär-Eiswirth model or the Fenton-Karma model (data not shown).

${ }^{8}$ It is chosen similar to the form that could be expected for example in a Fokker-Plank equation [100]. The fit has mostly problems with examples that would require an additional term with a longer time constant.
} 


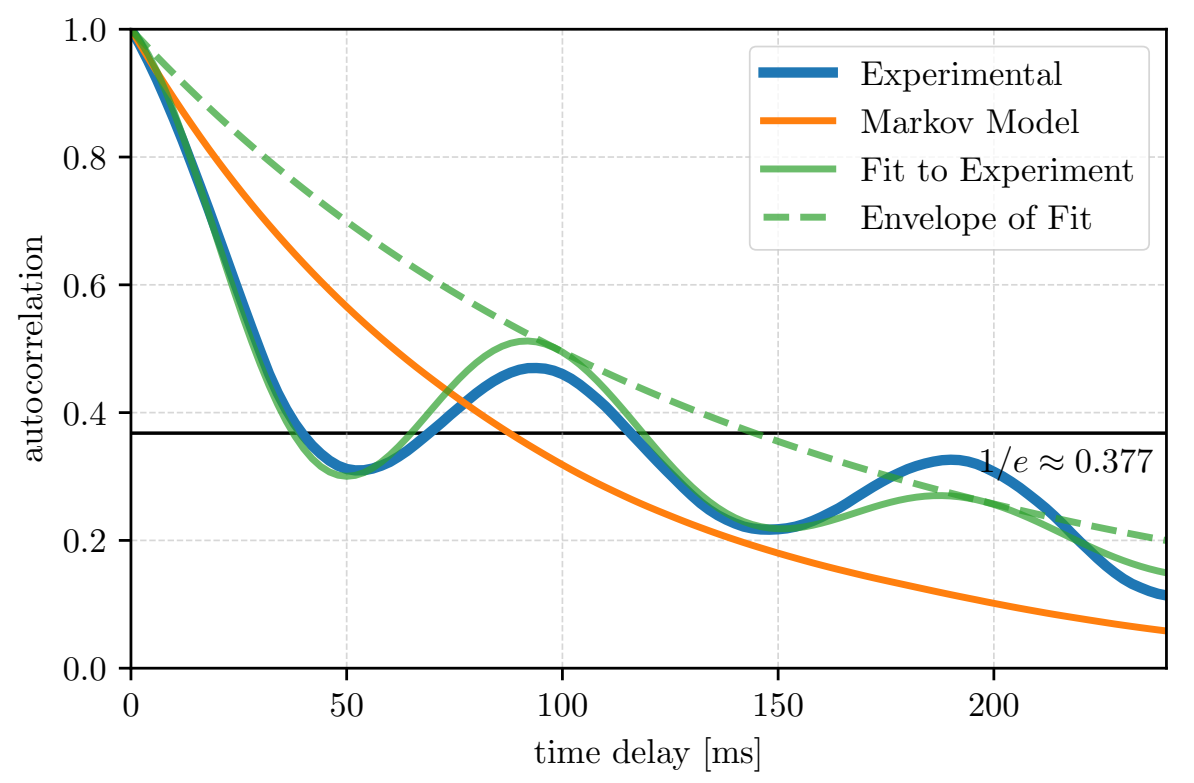

Fig. 6.8 Autocorrelation function of the NPS for the episode 2017-09-28 at 12:08 (30s of VF) calculated for delays of up to 120 frames or $240 \mathrm{~ms}$. The orange line represents the autocorrelation function of the Markov model simulated for one million frames (two million milliseconds) using the Gillespie algorithm. The green line indicates a fit to the experiment as described in the text (equation (6.4)). This specific longer video has a relatively slow falling autocorrelation function and a comparably poor fit.

with the fit parameters $\alpha_{1}, \alpha_{2}, \tau_{1}, \tau_{2}$, and the period. Although it has some difficulties, especially in the specific example, the fit allows to extract the time $\tau_{\text {exp }}$, at which the $\mathrm{ACF}$ drops to a value of $1 / e$. It further allows to estimate the period of the oscillatory component.

When calculating the ACF for time spans including many VF episodes, here, the $\mathrm{ACF}$ is estimated for each episode individually and then averaged. For short episodes of only three or five seconds, this may effectively filter long term correlations. In all ACF calculation a maximum delay of $240 \mathrm{~ms}$ is used (cf. 6.8).

Figure 6.9 (a) compares the decay times from the experiment $\left(\tau_{\text {exp }}\right)$ with those from Markov model simulations $\left(\tau_{\text {sim }}\right)$. This shows that, while the decay times vary widely and may have some systematic differences, their average values in experiments and simulations are similar and close to the typical CL.

Further, the period as extracted from the cosine component of the $\mathrm{ACF}_{\text {fit }}$ is similar albeit larger than the CL estimated from the video directly. While the origin of this offset is not clear, the individual CL and their comparison to the ACF oscillatory terms period are shown in Figure $6.9(\mathrm{~b})$. The black line denotes the identity. Except for the offset, the two values appear to be correlated.

These results also show that in most cases the ACF of the NPS drops significantly within about $100 \mathrm{~ms}$. An oscillatory component in the ACF with a similar period as the CL is expected due to repeating wave patterns. The decay time $\tau_{\exp }$ is smaller or 
a)

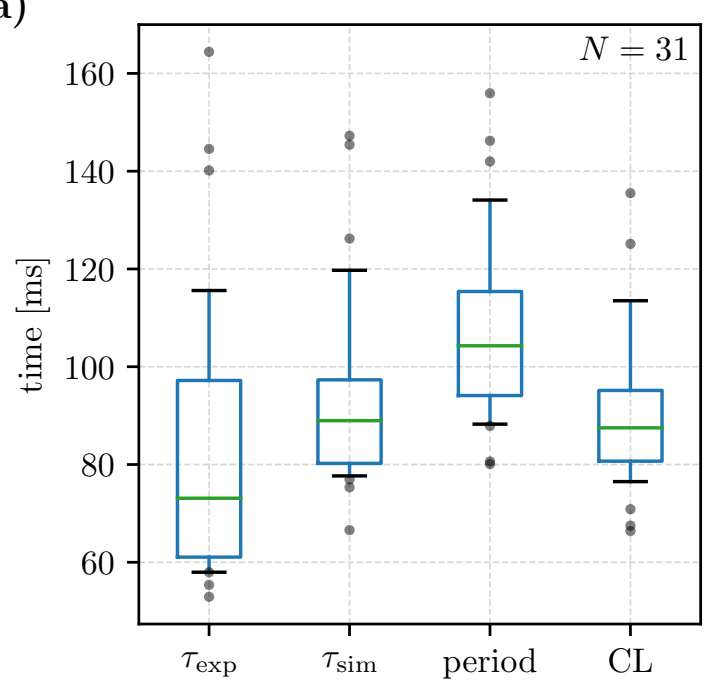

b)

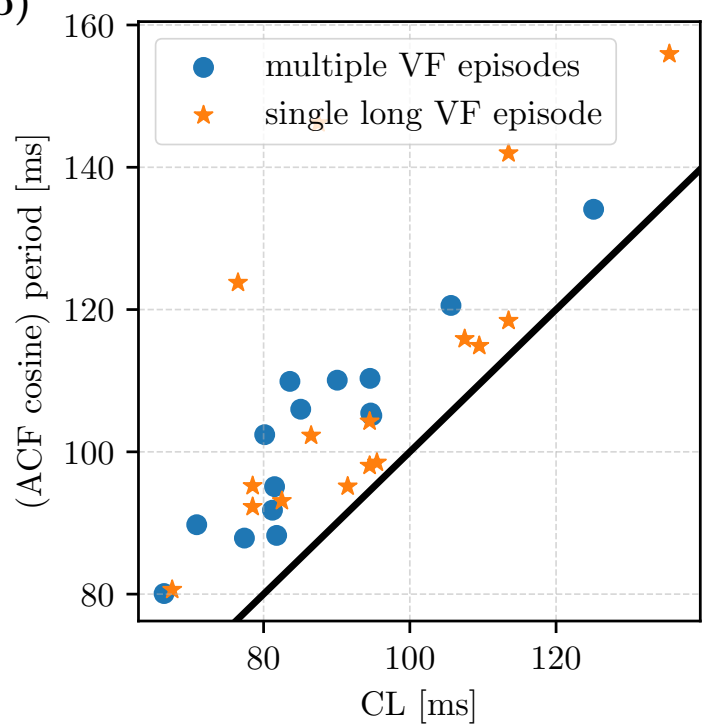

Fig. 6.9 a) A boxplot summarizing all analyzed time spans for the autocorrelation function of the NPS information. This includes the decay times observed in the experiment $\tau_{\exp }$ and estimated for its corresponding Markov model $\tau_{\text {sim }}$, as well as the period found by the fit in Equation (6.4). Further, the CL of the VF is given for comparison. b) Shows the average CL during fibrillation on the $x$-Axis and the estimated period from the ACF on the $y$-Axis. The colour indicates whether the data comes from a single long or many short VF episodes. The ACF for all data points are derived the same way as for Figure 6.8.

similar to the oscillatory period. Thus, while the oscillatory component is present, it is not dominant as seen by the decay in the ACF below $1 / e$ before a full period.

Another observation to note is that while these ACF capture the short term correlations, in some examples of longer VF episodes, such as the first one in Figure 6.8, long term correlations seem to exist. This gives an additional argument for the existence of intermittent behaviour which will be discussed in the next section.

\subsection{Nonstationarity and Intermittence}

In the above sections, time spans containing multiple VF episodes were analyzed. Between these VF episodes, or even within long VF episodes, the dynamics of the arrhythmia may change. For example, the dynamics of an arrhythmia is known to change over time after it was induced ${ }^{9}$. Thus, while the probability distributions for many episodes in Section 6.2 describe the observed NPS distribution well, the distribution may differ strongly for an individual episode. Intermittence, or variability, during VF episodes, such as a lower NPS were intensively studied in Alexander Schlemmer's $\mathrm{PhD}$ thesis [101] and approaches for quantification were also discussed in our paper [85].

\footnotetext{
${ }^{9}$ Although this effect is stronger in-vivo, rather in these ex-vivo experiments, because in-vivo the blood pumping stops during VF while in the experiment the perfusion remains unchange.
} 
Table 6.1 Comparison of how much the average NPS fluctuate over individual episodes compared to simulation results. The simulations are carried out with the same time spans and those parameters are fitted to the observed experimental rates. The standard deviation for the simulation results are calculated by simulating $100 \mathrm{VF}$ episodes with the same Markov model parameter set. The ratio column lists the ratio of both standard deviation columns highlighting the increased variability in the experiment.

\begin{tabular}{lrrrcccc}
\hline \multirow{2}{*}{ day } & & \multicolumn{2}{c}{ Experiment } & \multicolumn{2}{c}{ Simulation } & \\
\hline $2017-09-21$ & $11: 00-12: 30$ & 0 & 15.1 & 1.78 & 15.1 & 0.995 & 1.79 \\
& $19: 30-20: 20$ & 0 & 16.0 & 2.74 & 15.9 & 1.77 & 1.54 \\
\hline $2017-09-28$ & $14: 30-15: 10$ & 0 & 21.6 & 1.64 & 21.6 & 1.2 & 1.36 \\
\hline $2017-10-05$ & $13: 00-16: 00$ & 7 & 20.6 & 2.89 & 20.6 & 1.17 & 2.47 \\
& $16: 40-18: 50$ & 0 & 14.2 & 3.73 & 14.5 & 1.57 & 2.38 \\
\hline $2017-10-12$ & $16: 00-18: 10$ & 0 & 14.4 & 1.61 & 14.2 & 1.12 & 1.44 \\
\hline $2017-10-17$ & $16: 00-19: 15$ & 63 & 17.0 & 3.38 & 17.4 & 3.31 & 1.02 \\
& $20: 15-22: 10$ & 0 & 16.9 & 5.14 & 17.2 & 2.15 & 2.39 \\
\hline $2017-11-16$ & $12: 00-12: 38$ & 16 & 17.5 & 1.39 & 17.4 & 0.924 & 1.5 \\
& $13: 10-14: 20$ & 24 & 23.5 & 3.17 & 23.9 & 2.6 & 1.22 \\
& $14: 20-15: 00$ & 24 & 28.5 & 4.44 & 29.3 & 3.38 & 1.32 \\
& $16: 05-18: 50$ & 0 & 17.1 & 1.52 & 17.3 & 1.19 & 1.27 \\
\hline $2017-11-23$ & $18: 00-19: 50$ & 15 & 7.84 & 2.34 & 7.92 & 1.3 & 1.8 \\
\hline $2017-11-30$ & $12: 30-14: 45$ & 10 & 22.3 & 3.02 & 22.2 & 1.52 & 1.99 \\
& $16: 00-17: 50$ & 0 & 14.4 & 3.66 & 14.7 & 1.85 & 1.98 \\
\hline
\end{tabular}

Such variations are interesting also with respect to defibrillation shocks. Whether or not a shock is capable to defibrillate is a probabilistic event: with the same energy a VF is sometimes successfully terminated and sometimes not. Finding variations in the dynamical properties over the course of a VF may thus help to understand this probabilistic nature.

First, I wish to demonstrate that such fluctuations between VF episodes cannot be fully explained by the stochastic behaviour which is captured by the previously shown Markov model. Let us assume that a single short episode has constant dynamical properties. Additionally, the ACF has decay time $\tau_{\exp }$ which is comparable to that of the Markov model $\left(\tau_{\text {sim }}\right)$ as shown in Section 6.4. If these assumptions are true, using the Markov model to simulate VF episodes of the same length as the experimentally observed individual VF episodes should yield comparable statistics. In other words, if individual VF episodes are all comparable, the average NPS of each VF episode is expected to fluctuate in a similar manner as it does for the simulated episodes.

The result of how the average NPS fluctuates between VF episodes and between 100 simulated episodes of the same (average) length is shown in Table 6.1. Both for 
a)

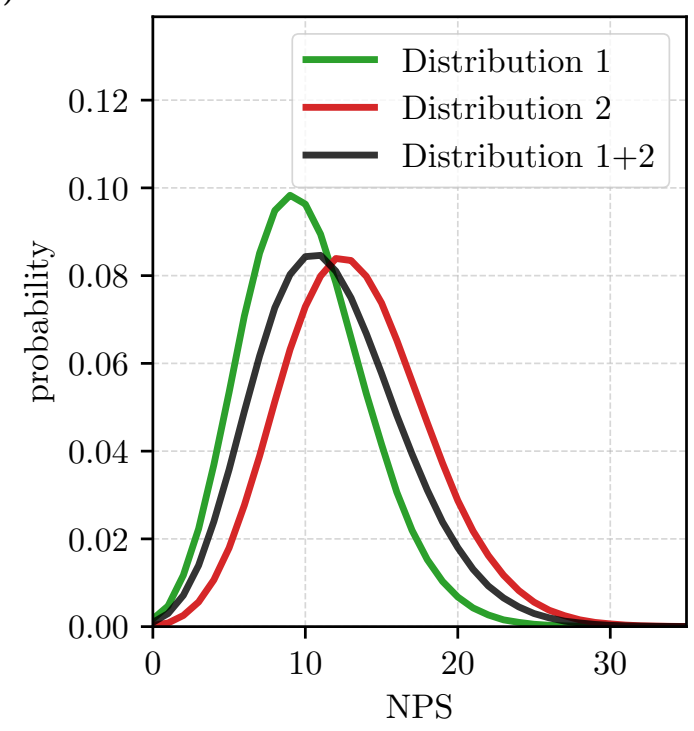

b)

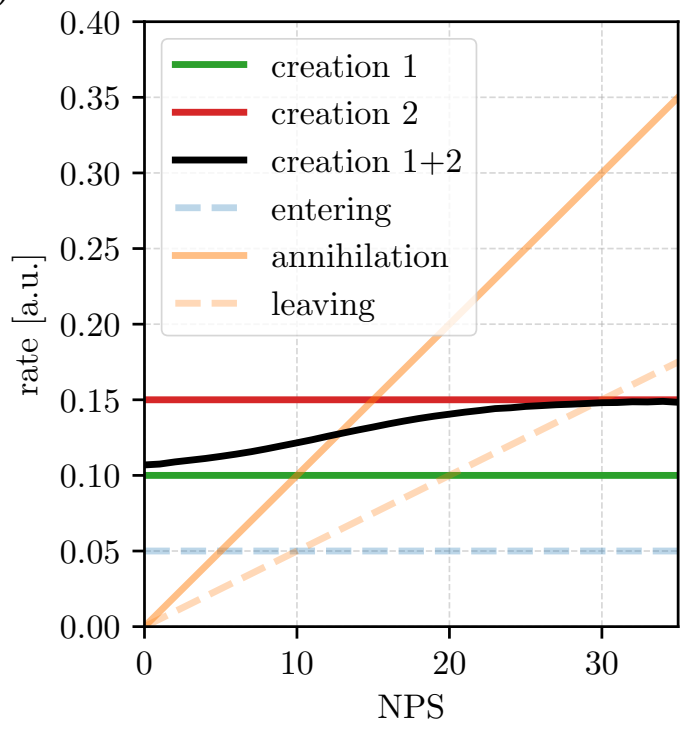

Fig. 6.10 a) Exemplary NPS probability distributions (1 and 2) based on the rates in (b). The distribution $1+2$ shows the average of distributions 1 and 2. b) Shows the corresponding rates creating the distributions, while only the creation rate differs. The creation rate that would be observed for the black distribution in (a) is also given. A model based on this would reproduce distribution $1+2$ in (a).

the simulation and experiment the mean and standard deviation of the average NPS over the individual episodes are shown. While the mean is known to be identical for the simulated episodes and the experimental episodes, the standard deviation of the mean NPS (of individual episodes) seen in the experiments is always larger than for the simulated episodes. This is true even though the time constant $\tau_{\exp }$ of the autocorrelation function tends to be slightly shorter than $\tau_{\text {sim }}$ (compare previous section) and a shorter time constant would mean a faster loss of correlation leading to a smaller standard deviation in the mean NPS.

Thus, it seems that a purely stochastic model of the NPS during VF cannot capture the full variability observed in the experiment. From the perspective of the Markov model, this can be associated with fluctuations of the model parameters over time. This is discussed in Figure 6.10, which shows the rates and associated distribution for two independent Markov models. In this example, they only differ in the creation rate. In Figure 6.10 (a) the black line indicates the result of combining both distributions with equal weight. Accordingly, Panel (b) shows the effective rate that would be measured when analyzing both together. Due to the property and type of the parameter change, the Markov model based on the combined creation $1+2$ rate has the exact same probability distribution as the directly averaged distribution shown in (a). This visualizes that the Markov modelling approach is robust under the assumption of slow and uncorrelated changes in the rates. Additionally, it provides a likely explanation for the higher standard deviation shown before. If multiple episodes have somewhat 
different rates as seen in the figure, their mean values will vary much stronger than would be expected from the combined distribution alone.

A model such as indicated in Figure 6.10 may help to approach the variability seen within VF episodes. If the heart switches between the behaviour associated with distribution 1 and 2 with a certain probability, a new Markov model could be created incorporating both. Instead of an average however, the new model would now be a hidden Markov model. This is because it is not known if the current NPS value comes from the behaviour of distribution 1 or from distribution 2, so that there are two states for the same observed NPS. However, for such hidden Markov models, efficient algorithms exist to find the most likely distribution based on the time series of NPS (see e.g. [98, Ch. 3.2]). Thus, in the future, such considerations may open a way to identify or classify the current state of a fibrillation also with respect to short term fluctuations. 



\section{Chapter 7}

\section{Spatial Phase Singularity Organization and Emergent Dynamics}

Spiral waves in cardiac tissue or chemical reactions are known to interact in complex ways with heterogeneities [102-104]. It is also known that regional differences in dynamic properties may induce arrhythmia and this has been shown in experiments [105]. While Valderrábano et al. showed that phase singularities (PS) cluster along geometrical heterogeneities such as blood vessels or trabeculae [102], the importance of dynamical heterogeneity - i.e. changes in the local dynamics rather than in geometry - on the spatial organization of spiral waves in fibrillation has not been studied in depth in experiments. Further, while simulation studies have addressed issues of spiral wave meandering in a dynamical heterogeneous medium, few have studied how such heterogeneities influence the spatio-temporal chaos associated with fibrillation.

A more detailed introduction can be found in the manuscript included as part of the following Section 7.1. This paper studies the changes due to regions of lower excitability embedded into a two dimensional medium modelled by the Bär-Eiswirth model. Such regions may be likened to ischemic conditions and, in this interpretation, compared to experimental findings.

After the simulation study in the following paper, I will validate the study by showing experimental findings of spatial organization of PS similar to those found by Valderrábano et al. [102]. However, unlike Valderrábano et al. my work analyzes the importance of dynamical heterogeneity. 


\subsection{Emergent Dynamics of Spatio-Temporal Chaos in a Heterogeneous Excitable Medium}

The following pages contain the article [106]. The paper is reproduced from:

P. Bittihn, S. Berg, U. Parlitz, and S. Luther. "Emergent dynamics of spatio-temporal chaos in a heterogeneous excitable medium". In: Chaos: An Interdisciplinary Journal of Nonlinear Science 27.9 (Sept. 1, 2017), p. 093931. DOI: 10.1063/1.4999604,

with the permission of AIP Publishing.

The original idea and the observation of emergent stable states on a regular grid were previously found as part of Philip Bittihn's PhD thesis. This initial finding included observation of stability changes with respect to the radius $R$. The further analysis of the effect of the heterogeneity on the chaotic dynamics to elucidate the contributing parameters and the effect of irregular heterogeneities was equally devised by Philip Bittihn and me in close cooperation.

The code to estimate the Lyapunov exponents as well as most of the general simulation framework was previously implemented by Philip Bittihn. All further simulation and data analysis code were done by me. I performed both the simulations and data analysis presented in the paper.

All figures shown in the paper were created by me. All authors contributed extensively to the writing of the manuscript, with the results section mainly written by me. 


\title{
Emergent dynamics of spatio-temporal chaos in a heterogeneous excitable medium
}

\author{
Philip Bittihn, ${ }^{1, a), b), c)}$ Sebastian Berg, ${ }^{1,2, b)}$ Ulrich Parlitz,, ${ }^{1,2,3}$ and Stefan Luther ${ }^{1,2,3,4,5}$ \\ ${ }^{1}$ Max Planck Institute for Dynamics and Self-Organization, Göttingen, Germany \\ ${ }^{2}$ Institute for Nonlinear Dynamics, Georg-August-Universität Göttingen, Göttingen, Germany \\ ${ }^{3}$ German Center for Cardiovascular Research (DZHK), Partner Site Göttingen, Göttingen, Germany \\ ${ }^{4}$ Institute of Pharmacology and Toxicology, University Medical Center, Göttingen, Germany \\ ${ }^{5}$ Department of Physics and Department of Bioengineering, Northeastern University, Boston, MA 02115, USA
}

(Received 31 March 2017; accepted 11 July 2017; published online 13 September 2017)

\begin{abstract}
Self-organized activation patterns in excitable media such as spiral waves and spatio-temporal chaos underlie dangerous cardiac arrhythmias. While the interaction of single spiral waves with different types of heterogeneity has been studied extensively, the effect of heterogeneity on fully developed spatio-temporal chaos remains poorly understood. We investigate how the complexity and stability properties of spatio-temporal chaos in the Bär-Eiswirth model of excitable media depend on the heterogeneity of the underlying medium. We employ different measures characterizing the chaoticity of the system and find that the spatial arrangement of multiple discrete lower excitability regions has a strong impact on the complexity of the dynamics. Varying the number, shape, and spatial arrangement of the heterogeneities, we observe strong emergent effects ranging from increases in chaoticity to the complete cessation of chaos, contrasting the expectation from the homogeneous behavior. The implications of our findings for the development and treatment of arrhythmias in the heterogeneous cardiac muscle are discussed. Published by AIP Publishing.

[http://dx.doi.org/10.1063/1.4999604]
\end{abstract}

Understanding the mechanisms that govern the onset, development, and termination of cardiac arrhythmias is essential to develop and further refine novel strategies for controlling them. However, cardiac tissue is inherently heterogeneous, and heterogeneity might be exacerbated in diseased hearts. In a generic model of excitable media, we investigate how patches of lower excitability change the characteristics of spatio-temporal chaos, which is related to lethal cardiac fibrillation. Surprisingly, the presence of multiple discrete heterogeneities may lead to the complete cessation of chaos, even though lower excitability increases complexity in a homogeneous medium. In other cases, the complexity of spatio-temporal chaos increases beyond the values expected from homogeneous behavior. Our results show that spatial variations in local parameters may have truly emergent effects and information about the distribution of natural heterogeneity in the cardiac muscle as well as the spatial scale and strength of pathological heterogeneities is an indispensable prerequisite for understanding the stability properties of cardiac arrhythmias and developing control strategies tailored to specific types of dynamics.

\section{INTRODUCTION}

Excitable media provide a generic description for a variety of spatially extended systems, including chemical and biological examples. ${ }^{1-3}$ A prominent example is the cardiac

\footnotetext{
a)pbittihn@ucsd.edu

${ }^{b)}$ P. Bittihn and S. Berg contributed equally to this work.

${ }^{c}$ Current address: BioCircuits Institute, University of California San Diego, La Jolla, California 92093, USA
}

tissue, where a single cell can be stimulated to display an action potential, and the communication through gap junctions and the extracellular space corresponds to diffusive coupling. The discovery that activation patterns found in excitable media such as stable or interacting rotating waves underlie cardiac arrhythmias ${ }^{4-7}$ has led to identification of cardiac arrhythmias as a "dynamic disease" that can be studied using computer simulations and the methods of nonlinear dynamics. In the past decades, more and more detailed mathematical models were developed that faithfully describe the ionic transmembrane currents of cardiac cells and the anisotropic bidomain structure of tissue (see, e.g., Clayton et al. ${ }^{8}$ for a review). However, since the fundamental dynamics underlying tachycardia and cardiac fibrillation can already be observed in homogeneous, generic models of excitable media, a spectrum of models with varying levels of detail continues to be used to uncover the underlying nonlinear phenomena which lead to and characterize cardiac arrhythmias. 9 The work presented in this paper uses the latter approach, building on the analogy between generic excitable media and cardiac tissue, while taking into account a prominent property of cardiac tissue, namely spatial heterogeneity in local tissue parameters.

The importance of tissue heterogeneity has been pointed out in various contexts in the past decades. Besides the known regional differences in electrical cell properties across the ventricular and atrial wall of the heart, ${ }^{10-12}$ tissue heterogeneity can be further increased by cardiac remodeling after myocardial infarction $^{11}$ and during reperfusion of the ischemic tissue which may lead to reductions in tissue excitability. ${ }^{13}$ Lower excitability has been implicated in the proarrhythmic effects of certain antiarrhythmic drugs. ${ }^{14}$ Fibroblasts have 
also been suggested as a major source of tissue heterogeneity, as they modulate action potential propagation and can act as current sinks, ${ }^{15,16}$ and fibrosis has been directly implicated in the development of cardiac arrhythmias. ${ }^{17,18}$ In modeling studies, it was found that the arrhythmogenenic effect of varying degrees of fibrosis is determined by the maximum local fibrosis, ${ }^{19}$ assuring that the properties of arrhythmias cannot be understood in terms of average tissue properties. A study by Shajahan et al. showed that spiral wave dynamics in different mathematical models of cardiac tissue depends sensitively on the presence and position of both conduction and ionic heterogeneities $^{20}$ and the importance of conduction heterogeneities has been considered both for the creation of spiral wave reentry ${ }^{21}$ and the spontaneous ${ }^{22}$ as well as the forced ${ }^{23}$ termination of arrhythmias.

More abstract modeling studies have tried to build a bottom-up understanding of the influence of heterogeneities by investigating the interaction of local variations in parameters with the constituents of many cardiac arrhythmiasreentrant waves. After the stability properties of spiral waves and their potential to deviate from the simple dynamics of rigid rotation ("meandering") had been established using the tools of nonlinear dynamics, ${ }^{24-26}$ more recent studies have extended the stability concept to spirals subjected to external stimuli or evolving in media with localized heterogeneities using the so-called response functions. ${ }^{27,28}$ They found that spiral waves may be attracted or repelled by circular heterogeneities, and abrupt spatial changes in medium parameters may also lead to a heterogeneity-induced drift along the boundary of the heterogeneity or orbiting around circular heterogeneities, which is in line with earlier experimental results on artificially induced regions of lower excitability in cardiac tissue. ${ }^{29}$ Similar effects have also been observed for other types of heterogeneities such as impermeable inclusions (i.e., holes) in the medium. ${ }^{30}$ In different models of excitable media, heterogeneity was shown to cause drift of spiral waves towards regions of longer APD and lower excitability, ${ }^{31-34}$ although exceptions of the so called "anomalous drift" are also known. ${ }^{35}$

Most of the above studies were concerned with the effect of heterogeneity on the stability of single reentrant waves or the likeliness for the onset of arrhythmias in the presence of heterogeneity. The characterization of these instabilities is important to understand the susceptibility of the cardiac substrate to the development of spiral wave breakup and thus life-threatening arrhythmias. However, the question of how heterogeneities can shape the quality and complexity of spatio-temporal chaos itself has received less attention, although the answer might be equally important for informing more specific and tailored termination strategies for undesired cardiac rhythms. Here, we use the Bär-Eiswirth model of spiral wave breakup ${ }^{36}$ and consider heterogeneities in the local excitability of the medium. More specifically, we limit ourselves to parameters which, in homogeneous substrates, all lead to spatio-temporal chaos, i.e., which do not differ qualitatively on a superficial level. We build on the characterization by Strain and Greenside, who showed that, at low excitabilities, chaos is extensive and sustained for large enough spatial domains. ${ }^{37}$ We characterize the dynamics using tools from nonlinear dynamics - the Lyapunov dimension-and phase singularity statistics, allowing us to systematically investigate how heterogeneities in excitability of different strengths, scales, and shapes can alter the dynamics and to detect effects that deviate from the expectation based on average parameters. First, we characterize the dynamics in homogeneous media for a range of excitabilities, before we combine different parameters to obtain heterogeneous spatial structures that range from random to spatially ordered.

\section{METHODS}

\section{A. Model and numerical solution}

We use the generic model of excitable media by Bär and Eiswirth $^{36}$ with the following equations:

$$
\begin{aligned}
\frac{\partial u}{\partial t}=\frac{1}{\varepsilon} \cdot u \cdot(1-u) \cdot\left(u-\frac{v+b}{a}\right) & +\nabla^{2} u, \\
& = \begin{cases}-v & u<\frac{1}{3} \\
1-6.75 \cdot u \cdot(u-1)^{2}-v & \frac{1}{3} \leq u \leq 1 \\
1-v & u>1,\end{cases}
\end{aligned}
$$

which is an extension of the well-known Barkley model ${ }^{38}$ and supports spatio-temporal chaos via at least two different breakup mechanisms. ${ }^{39}$ Here, we use the parameters $a=0.84$ and $b=0.07$, that lead to Doppler-induced spiral wave breakup near the core for $\varepsilon \gtrsim 0.069,{ }^{36}$ which is a type that is also seen in many cardiac models. ${ }^{9}$ The homogeneous background value for the excitability parameter is $\varepsilon=0.08$ throughout this study when heterogeneities with a value of $\varepsilon=\varepsilon_{h}$ are investigated. Note that here larger $\varepsilon$ corresponds to lower excitability, since the upstroke velocity of the fast $u$ variable varies with $\varepsilon^{-1}$. Strain and Greenside found that spatio-temporal chaos beyond the critical value of $\varepsilon$ is longlived and statistically stationary in large enough twodimensional systems with periodic boundary conditions, ${ }^{37}$ enabling estimation of the Lyapunov dimension and providing a well-defined basis for other statistical measures we put forward in this study.

It should be noted that our very broad usage of the term "excitability" to refer to the parameter $\varepsilon^{-1}$ of the Bär-Eiswirth model is, to some extent, a linguistic simplification that should be taken with a grain of salt in terms of cardiac electrophysiology. While the primary effect of a change in $\varepsilon$ is indeed a change in upstroke velocity (roughly corresponding to changes in cardiac sodium channel opening dynamics), its impact is not limited to the initial phase of an activation, as it determines the overall time scale separation between the fast $u$ and the slow recovery variable $v$. For example, a further increase of $\varepsilon$ beyond the range of parameter values investigated in this study might qualitatively change the behavior of the model, not necessarily leading to conduction block in the cardiac-tissue sense. The word "excitability" will be used despite these complications to provide the closest physiological analogy for the kind of heterogeneity we investigate. 
Except where otherwise noted, simulations are carried out in a two-dimensional rectangular domain of size $40 \times 40$ with periodic boundary conditions, using a forward-time centered space finite difference scheme with a spatial step of $\Delta x=1 / 3$ and a time step of $\Delta t=2 \times 10^{-3}$. The simulation period is 10000 time units and statistical analysis is carried out on the final 5000 time units. The initial condition is randomly generated to produce a few spiral waves in random locations that subsequently break up and produce spatiotemporal chaos. We confirmed that the results do not depend on the concrete choice of initial condition as long as the medium does not immediately return to the stable, spatially homogeneous quiescent state.

To obtain randomly shaped heterogeneities in the excitability parameter $\varepsilon$ that occupy $x \%$ of the medium, identically distributed Gaussian random numbers are generated for every computational point in the domain. These values are then smoothed with a Gaussian spatial filter of standard deviation $\sigma$. The computational points are sorted by the value of the resulting field, and the parameter $\varepsilon$ is set to $\varepsilon_{h}$ instead of 0.08 at the top $x \%$ points on this sorted list, yielding patches of $\varepsilon_{h}$ in a $\varepsilon=0.08$ background that occupy $x \%$ of the space and whose size correlates with $\sigma$.

\section{B. Characterization of complexity}

To compute the Lyapunov dimension $D_{L}$, the dynamics are first simulated for 8300 time units to account for any transient dynamics. We verified that the results do not differ from those obtained with only 1500 time units equilibration time (roughly 300 spiral periods; data not shown). Random vectors in tangent space are then initiated and integrated using the linearized version of (1). The linearization is carried out for the local dynamics and vectors are defined on the same spatial grid as the full dynamics. The diffusion term with periodic boundary conditions in (1) is preserved in tangent space. ${ }^{40}$ Gram-Schmidt orthonormalization of tangent space vectors is done every 1 time units. The vectors are allowed to align with the dynamics for another 200 time units before Lyapunov exponents $\lambda_{i}$ with $\lambda_{1}>\lambda_{2}>\cdots>\lambda_{n}$ are calculated using the standard Benettin procedure for the remaining 1500 time units. ${ }^{41,42}$ For all simulations carried out in this study, we integrate 120 vectors in tangent space alongside the actual dynamics, yielding $n=120$ Lyapunov exponents. The Lyapunov or Kaplan-Yorke dimension is then calculated using the standard formula

$$
D_{L}=j+\frac{1}{\lambda_{j+1}} \sum_{k=1}^{j} \lambda_{k}
$$

where $j$ is the largest index for which $\sum_{k=1}^{j} \lambda_{k}$ is still positive.

Phase singularities (PS) are detected by calculating a local phase

$$
\phi(x, y)=\arctan 2\left(u(x, y)-u^{*}, v(x, y)-v^{*}\right),
$$

at each computational point, where $\arctan 2(u, v)$ is the twoargument arctan function that maps each point to its angle $-\pi<\phi \leq \pi$ in the $u-v$ plane. We use an origin in phase space placed at $u^{*}=0.65, v^{*}=0.45$, and integrate the phase difference between consecutive points around a 4-point square path. A phase singularity is present if the integral is $\pm 2 \pi$ as opposed to 0 , where the sign determines the chirality. For the smooth simulation data of the Bär-Eiswirth model, the longer 8-point path that avoids large phase jumps due to nonlinearities in the model or experimental noise is not necessary. ${ }^{43}$ While an optimal origin in phase space could be chosen for each parameter set, this would lead to ambiguities in the case of heterogeneous parameters in the spatial domain. Therefore, we use the origin above across all parameters investigated in this study. To calculate spatial phase singularity densities, each computational point is considered a "bin" and the number of phase singularities present in each bin is counted over the final 5000 time units of a 10000 time unit simulation. Numerically, we calculate

$$
\rho_{\mathcal{N}}(x, y)=40^{2} \cdot \frac{\mathcal{N}(x, y)}{N_{t} \Delta x^{2}},
$$

where $\mathcal{N}(x, y)$ is the total number of phase singularities present at the computational point $(x, y)$ across $N_{t}$ time points and $\Delta x$ is the spatial numerical step size. The factor $40^{2}$ is arbitrary, but fixed, and leads to typical values of $\rho_{\mathcal{N}}$ between 0 and 100 . As $40 \times 40$ is also our standard domain size, a convenient side effect of this scale is that the local measure $\rho_{\mathcal{N}}(x, y)$ can be compared directly to the total number of phase singularities in the standard domain.

As an alternative measure for chaoticity or turbulence besides the Lyapunov dimension, we introduce the phase singularity volatility or PS volatility $\mathcal{V}$. It is equal to the average rate of change of the number $\mathcal{N}$ of phase singularities. Since $\mathcal{N}$ is an integer variable

$$
\mathcal{V}=\frac{1}{T} \int_{T_{1}}^{T_{2}}\left|\frac{\mathrm{d} \mathcal{N}}{\mathrm{d} t}\right| \mathrm{d} t
$$

is actually an integral over a comb of Dirac $\delta$ distributions, and we numerically calculate it by summing the absolute differences of $\mathcal{N}$ between the beginning and end of small time intervals

$$
\mathcal{V}=\frac{1}{T_{2}-T_{1}} \sum_{i=0}^{N-1}\left|\mathcal{N}\left(T_{1}+(i+1) \Delta t\right)-\mathcal{N}\left(T_{1}+i \Delta t\right)\right|
$$

where $\Delta t=\left(T_{2}-T_{1}\right) / N$. Each term in the sum will either be 0 (no change) or 2 (annihilation or creation of an opposite-chirality pair of phase singularities), provided $\Delta t$ is small enough such that each interval contains at most a single such event. Throughout this study, we use $\Delta t=2 \times 10^{-3}$ (i.e., the same as the simulation time step). Using a larger time $\Delta t$ in (6), leads to an increased probability for multiple creation/annihilation events per interval and an underestimation of $\mathcal{V}$. The volatility analysis for all simulations in this study was also carried out using intervals as large as $4 \times 10^{-2}$, and no changes in qualitative behavior were observed, while absolute numbers for $\mathcal{V}$ were significantly lower. 
Additionally, we track phase singularities to determine their individual lifetimes. This is done by minimizing the total distance traveled by phase singularities between consecutive time steps, assuming our time resolution does not allow for large jumps. Candidates for matching a specific phase singularity are either phase singularities of the same chirality in the next snapshot or those of opposite chirality within the same snapshot. The former identifies a movement and the latter represents either the annihilation (current snapshot) or creation (next snapshot) of a PS pair. This procedure allows us to define the lifespan $\mathcal{L}$ for each phase singularity in a simulation. We then calculate the average $\langle\mathcal{L}\rangle$ and the coefficient of variation $\mathrm{CV}(\mathcal{L})$ to characterize a particular simulation. Statistics of these measures across different realizations (usually 20, see below) are then obtained as usual. Note that $\langle\mathcal{L}\rangle, \mathcal{V}$ and $\mathcal{N}$ are related via $\langle\mathcal{L}\rangle=2 \mathcal{N} / \mathcal{V}$ for large enough averaging time $T=T_{2}-T_{1}$, as each PS makes two unit contributions to the volatility (one upon creation and one upon destruction), and the average number $\mathcal{N}$ of phase singularities is equal to $1 / T \cdot \sum \mathcal{L}$, where $\sum \mathcal{L}$ is the sum of the life spans of all PS. Therefore the additional information from $\mathcal{L}$ is contained in its distribution.

The very small but finite probability for spatio-temporal chaos to self-terminate ${ }^{37,60}$ combined with the extremely long simulations used here to obtain reliable statistics - with 10000 time units corresponding to well above 1500 dominant periods-leads to a small fraction of simulations that do not display sustained chaos for the entire simulation time. Instead, all activity is terminated and the medium goes to the stable quiescent state. Strain and Greenside noted that the dynamics before self-termination is statistically stationary and indistinguishable from sustained chaos in other simulations, ${ }^{37}$ which was recently confirmed by the identification of these termination events as abrupt type-II supertransients by Lilienkamp et al. ${ }^{60}$ Since our goal is to characterize spatio-temporal chaos, we therefore, for simplicity, excluded these simulations from the analysis where appropriate. Even for 10000 time units, spiral wave turbulence self-terminated in less than $0.4 \%$ of the cases, such that no such exclusion was necessary for most of the data points, which are usually averages of 20 simulations.

\section{RESULTS}

\section{A. Homogeneous excitability}

First, we establish a baseline for spatially homogeneous media exhibiting spatio-temporal chaos. Figure 1 shows typical snapshots of the system for two different $\varepsilon$ and the corresponding time series of the number of phase singularities $\mathcal{N}$. Higher $\varepsilon$ (lower excitability) leads to more turbulent dynamics causing less clear spiral cores and stronger fluctuations in the number of phase singularities $\mathcal{N}$.

To quantify these observations, Fig. 2 shows the Kaplan-Yorke dimension $D_{L}$, the PS volatility $\mathcal{V}$ [see (6)], the time-averaged number of phase singularities $\mathcal{N}$, and statistics of the lifespan $\mathcal{L}$ as a function of the excitability parameter $\varepsilon$. Because the calculation of the Lyapunov dimension is computationally intensive, $D_{L}$ was only calculated over a shorter period of time on a coarser $\varepsilon$ grid. The values of $D_{L}, \mathcal{V}$ and $\mathcal{N}$ obtained from these simulations are plotted a)

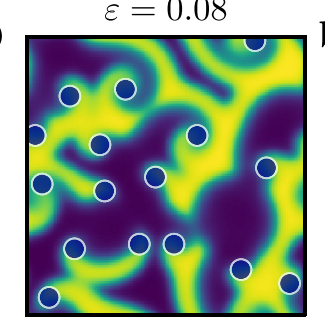

b)

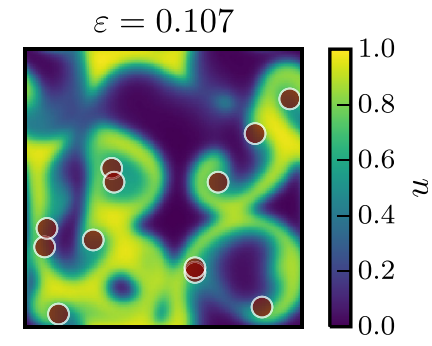

c)
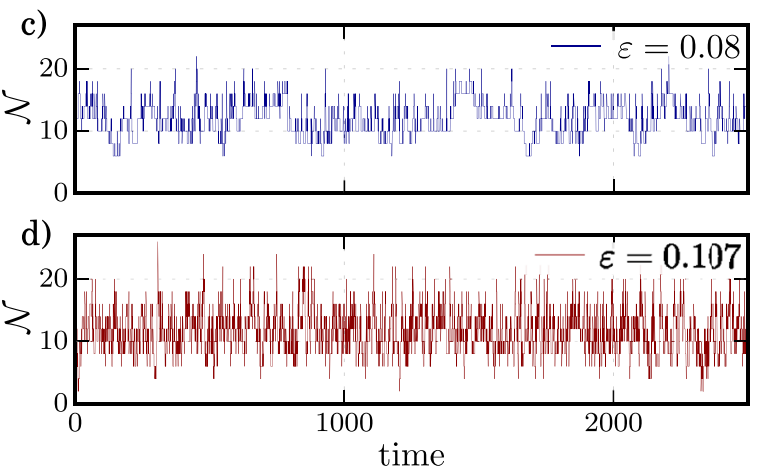

FIG. 1. (a) and (b) Snapshots of the $u$ variable for the homogeneous system with phase singularities marked as circles for two different values of $\varepsilon$. (c) and (d) Evolution of the number of phase singularities over time for the same values of $\varepsilon$. The segments have a volatility of $\mathcal{V}=1.3$ and $\mathcal{V}=4.2$.

as error bars for comparison with the finer scan for which only $\mathcal{V}, \mathcal{N}$ and lifespan statistics were calculated (displayed as shaded areas). Note that the number of phase singularities as detected by the method defined in Sec. II B, to a degree, depends on the origin in phase space which was chosen
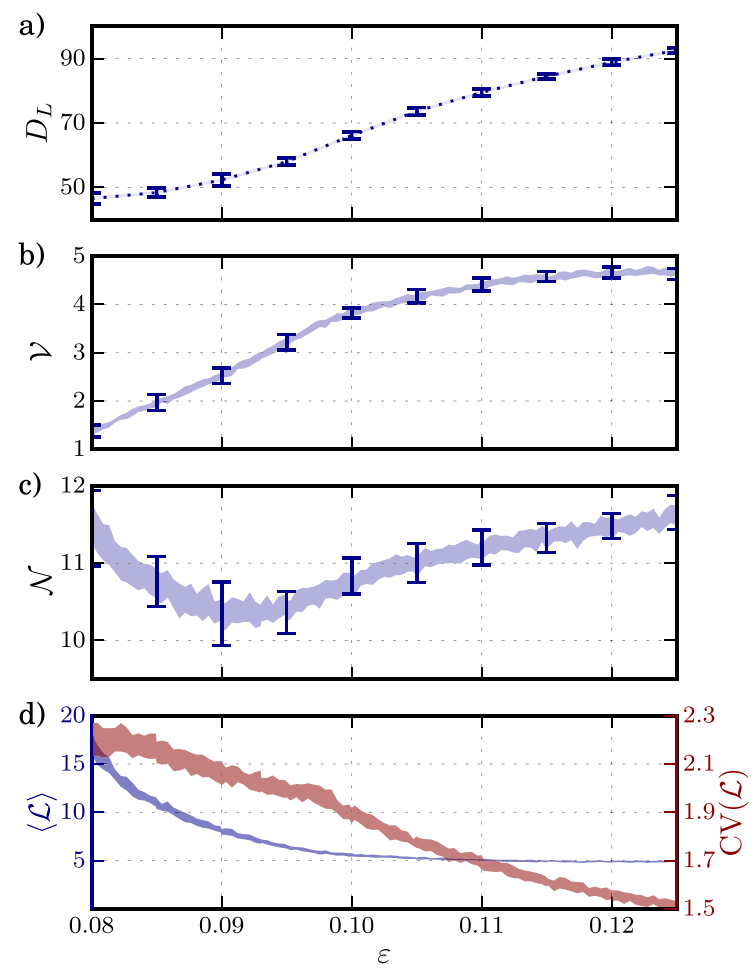

FIG. 2. (a) Lyapunov dimension $D_{L}$, (b) PS volatility $\mathcal{V}$, (c) number of PS $\mathcal{N}$, and (d) lifespan statistics in the homogeneous system when varying the excitability $\varepsilon$. The error bars shown are based on 20 simulations each averaging 1500 time units. The shaded areas in (b), (c), and (d) denote the value and standard deviation when averaging 5000 time units over 20 simulations. 
independent of the parameters. Therefore, the quantitative results could differ from those reported by Strain and Greenside. ${ }^{37}$ It is interesting to note that while the average $\mathcal{N}$ does not vary strongly between the parameters, both the Kaplan-Yorke dimension and the volatility increase substantially with $\varepsilon$, with $D_{L}$ almost doubling and the volatility increasing to almost five times its original value. Therefore, even beyond the onset of chaos at $\varepsilon \gtrsim 0.069$, complexity increases substantially for increasing $\varepsilon$. Due to the weak dependence of $\mathcal{N}$ on the excitability, even a normalized volatility "per phase singularity" $\mathcal{V} / \mathcal{N}$ would show the same dependence on $\varepsilon$ as that of the absolute volatility $\mathcal{V}$ in Fig. 2(c). This change in the dynamics of individual phase singularities is also reflected in a decrease of the expected lifespan of phase singularities as shown in Fig. 2(d). Interestingly, the lifespan's coefficient of variation also decreases continuously, indicating that the distribution of lifespans becomes more peaked at the same time.

Based on Fig. 2, $\mathcal{V}$ seems to be a suitable indicator of complexity when compared to the Lyapunov dimension $D_{L}$. Figures 3(a) and 3(b) show the spatial autocorrelation and the power spectrum for four equally spaced values of $\varepsilon$ which will be inspected more closely in the course of this study. It
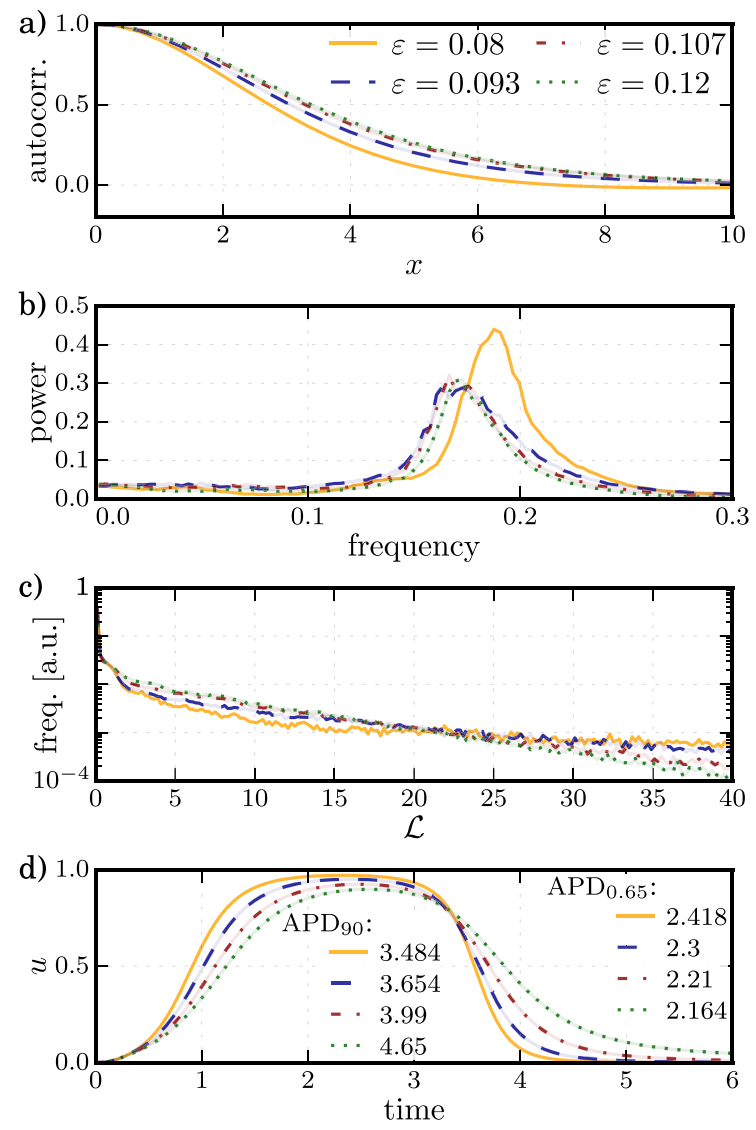

FIG. 3. Spatio-temporal characteristics of activity for four specific values of $\varepsilon$. (a) The spatial autocorrelation function for the four homogeneous cases calculated from the $u$ field and averaged over all time points. (b) The corresponding power spectrum, calculated from the time series of $u$ and averaged over all points in the spatial domain. (c) Relative frequencies of PS lifespans. (d) Time course of the model variable $u$ for a plane wave initiated in a quiescent medium. Indicated action potential durations (APDs) are measured for $90 \%$ repolarization and for a fixed threshold of $u=0.65$. is worth noting that most of the change happens between $\varepsilon=$ 0.08 and $\varepsilon=0.093$ : all three lower excitabilities (higher $\varepsilon$ ) have a lower dominant frequency and higher correlation length, while the difference among them is minor (and the trend for the dominant frequency is even reversed at $\varepsilon=0.12$ ). On a superficial level, the drop in frequency for larger $\varepsilon$ is expected, since the speed of the fast variable $u$ of the model in (1a) is inversely proportional to $\varepsilon$. However, this also means that the higher complexity illustrated by Fig. 2 is not due to generally faster dynamics but rather caused by altered creation and annihilation dynamics of phase singularities, which is confirmed by the distributions of PS lifespans shown in Fig. 3(c). Taken together, the general dominance of short-lived phase singularities and the existence of fewer phase singularities with long lifespans for lower excitability also explain the simultaneous decrease of the average lifespan and its coefficient of variation observed in Fig. 2(d), as the main effect of increasing $\varepsilon$ seems to be a reduction in the tail of the lifespan distribution. It should be noted that the local activation dynamics is naturally affected by the change in excitability. For example, the shape of the action potential, and consequently its duration, changes as $\varepsilon$ is increased [see Fig. 3(d)].

\section{B. Randomly structured heterogeneities}

We first consider the case of random patches of lower excitability that were constructed as described in Sec. II A. Figure 4 shows exemplary dynamics for two heterogeneous systems that both have a lower excitability of $\varepsilon_{h}=0.12$ in $40 \%$ of the area, but arranged on different spatial scales $\sigma$.

The first sign of emergent behavior is visible in Figs. 4(c) and 4(d). We find that just inside the lower excitability regions, the likelihood of finding PS is significantly increased, while just outside there is a depletion zone of PS. In Fig. 4(d) it also appears that small, but not too small, areas (such as in the lower right corner) which are surrounded by higher excitability on multiple sides seem to be more strongly affected. Likewise, in Fig. 4(c), the depletion effect also appears stronger for high excitability areas surrounded by low excitability.

To quantify this effect, we performed longer simulations in a domain of increased size where the excitability was reduced in an entire half plane, resulting in a linear interface in the middle of the medium. These results are shown in Fig. 5. The average PS density increases to a maximum at about 1.5-2 length units inside the less excitable area while it decreases on the other side, although Fig. 5(c) also shows a small long-range effect that causes slow convergence of the PS density to its homogeneous value for $\varepsilon=0.08$ towards the left. However, the dominant PS clustering as well as depletion dynamics happen on a characteristic length scale of 3 to 4 length units, and the strength of the effect increases with the height of the excitability step at the boundary. Therefore, the stronger clustering and depletion effects for some regions enclosed by an excitability boundary seen in Fig. 4 are potentially due to a length scale match with the clustering and depletion zones close to the boundary. An additional effect can be noticed when comparing the actual 
a)

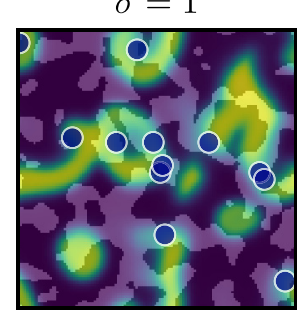

c)

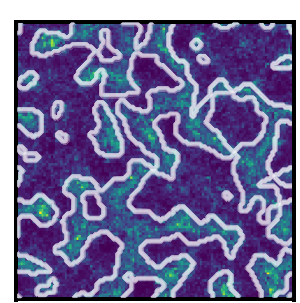

b)

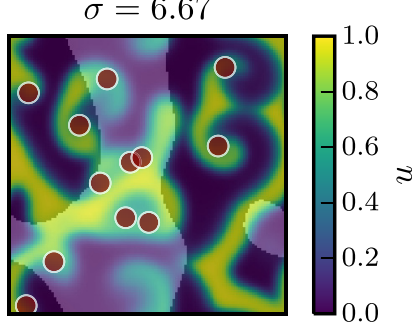

d)

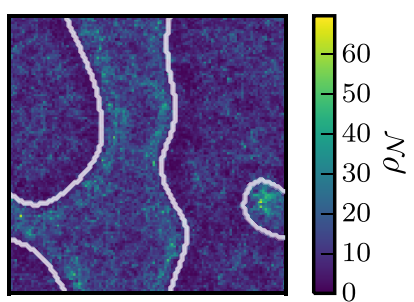

e)
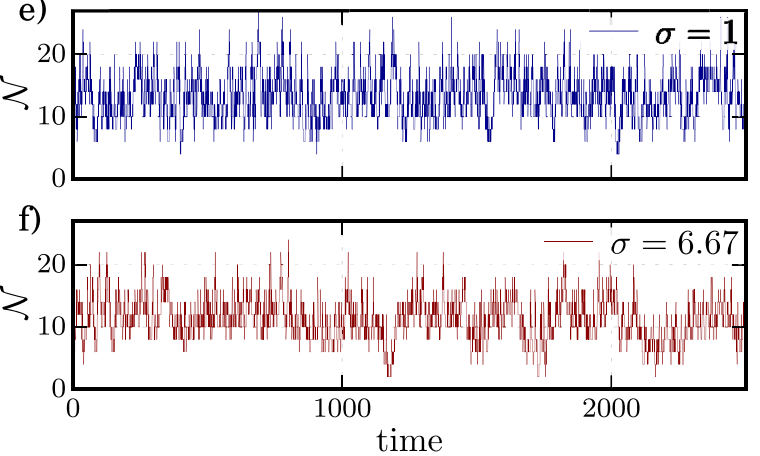

FIG. 4. Dynamics in two simulations for random patches of lower excitability $\left(\varepsilon_{h}=0.12\right)$ occupying $40 \%$ of the area but different spatial scale $\sigma$ (see Sec. II A). (a) and (b) Snapshots at $t=7500$ with PS marked by circles, the lighter areas correspond to lower excitability. (c) and (d) PS density per simulation domain calculated from $t=5000$ to $t=10000$ (excitability boundaries indicated by lines). (e) and (f) Temporal development of $\mathcal{N}$ before the snapshot. The segments have a volatility of $\mathcal{V}=4.1$ and $\mathcal{V}=2.6$.

number of PS in the simulation domain to the expected value calculated from PS densities for the corresponding homogeneous parameters in the left and right half plane, as done in Fig. 5(d): overall, the presence of the interface reduces the number of phase singularities and the suppression effect is stronger for higher values of $\varepsilon_{h}$.

We systematically investigated the complexity of the dynamics in heterogeneous media with random excitability patches as shown in Fig. 4 by measuring the PS volatility $\mathcal{V}$. While Figs. 6(a) and 6(b) visualize the raw value of $\mathcal{V}$, we also calculated a normalized volatility $\mathcal{V} / \mathcal{V}_{E} . \mathcal{V}_{E}$ is the expected volatility, assuming the homogeneous cases for $\varepsilon=0.08$ and $\varepsilon_{h}$ fully determine the volatility of the heterogeneous case, each contributing according to the area they occupy in the medium, i.e., $\mathcal{V}_{E}=\left[A_{\varepsilon=0.08} \mathcal{V}(\varepsilon=0.08)\right.$ $\left.+A_{\varepsilon=\varepsilon_{h}} \mathcal{V}\left(\varepsilon=\varepsilon_{h}\right)\right] / A_{\text {total }}$. Figures 6(c) and 6(d) show that, for large $\sigma$, i.e., large patches, the volatility agrees very well with this prediction, which is intuitive as locations far away from the boundary should not feel the interface between excitabilities. Hence, the general increase of $\mathcal{V}$ towards higher percentages of lower excitability in Figs. 6(a) and 6(b) is simply a result of the larger fractional area with lower excitability, which has a higher volatility according to Fig. 2.

a)

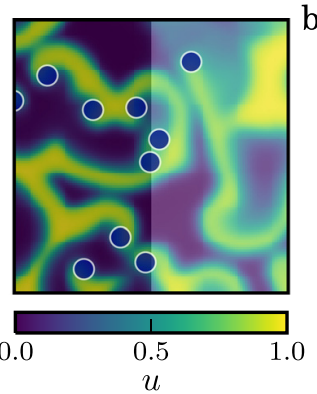

b)
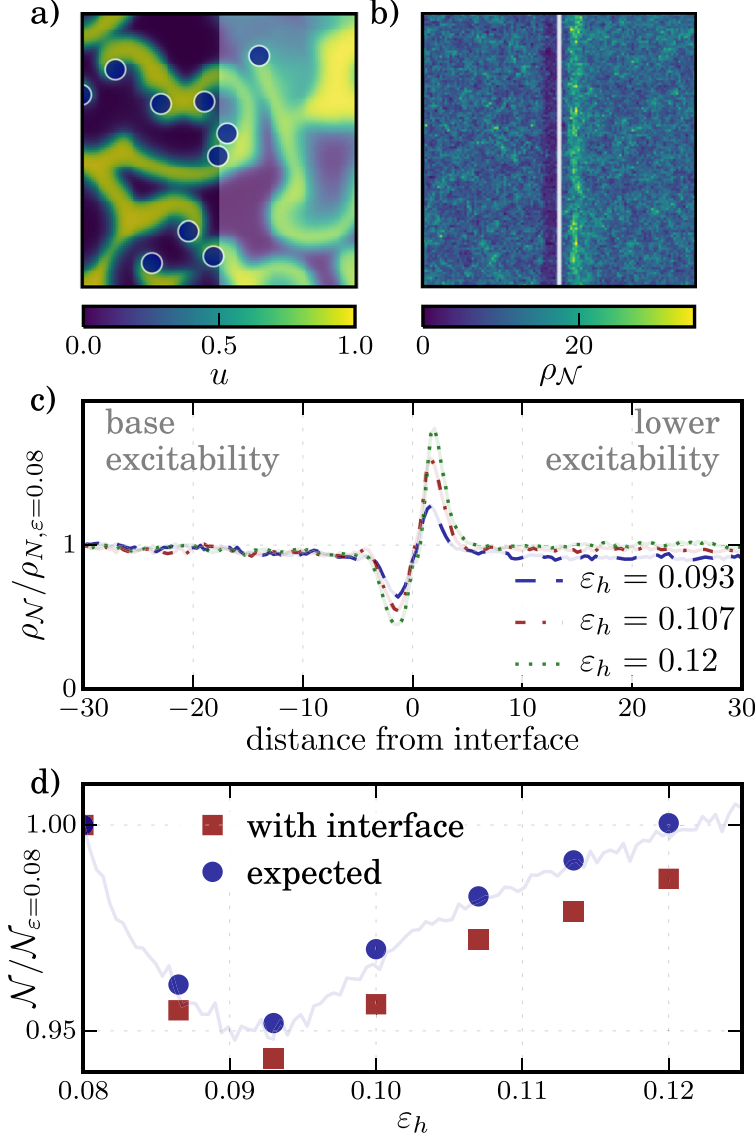

FIG. 5. Effects on PS statistics around an interface between basal $(\varepsilon=0.08$, left side) and lower excitability (right side). Simulations were carried out in a larger spatial domain of $160 \times 160$ spatial units, i.e., $480 \times 480$ computational points, to exclude boundary effects. (a) and (b) Snapshot at the end of the simulation and PS density close to the boundary calculated from $t=5000$ to $t=20000$ with the right side at $\varepsilon_{h}=0.12$. (c) PS density close to the excitability boundary (averaged along the $y$ direction) normalized by the PS density for the homogeneous case $(\varepsilon=0.08)$ for different values of the excitability in the right half of the medium. (d) Total number $\mathcal{N}$ of PS in the domain normalized by the number of PS in a homogeneous medium with $\varepsilon=0.08$ (red squares) for different excitabilities $\varepsilon_{h}$ in the right half of the domain. Expected number of PS calculated from homogeneous simulations with $\varepsilon=0.08$ and $\varepsilon=\varepsilon_{h}$ according to $\left[\mathcal{N}(\varepsilon=0.08)+\mathcal{N}\left(\varepsilon=\varepsilon_{h}\right)\right] / 2$ (blue circles) and the same estimate calculated from the homogeneous data measured in the original $40 \times 40$ domain (light blue line; based on data from Fig. 2(c) indicate minimal influence of domain size on homogeneous $\mathcal{N}$ and an overall reduction of $\mathcal{N}$ due to the presence of the interface.

However, close to $\sigma=1$, we find a large increase in $\mathcal{V}$ relative to $\mathcal{V}_{E}$, meaning that the number of phase singularities fluctuates more strongly than expected simply from the combination of the two homogeneous cases. While smaller patches of lower excitability attract phase singularities and cause a strong preferred localization inside those regions (cf. Fig. 4), they also seem to act as disturbing elements that cause more creation and annihilation events among phase singularities.

\section{Randomly placed circular heterogeneities}

The results above indicate that heterogeneities in excitability can have particularly strong effects exceeding the expectation based on homogeneous results, when enrichment 


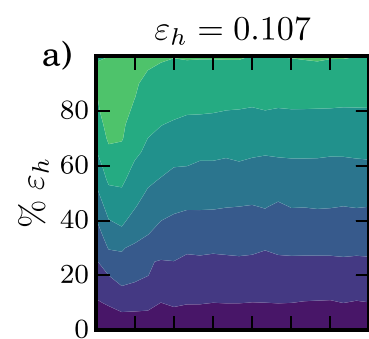

b)
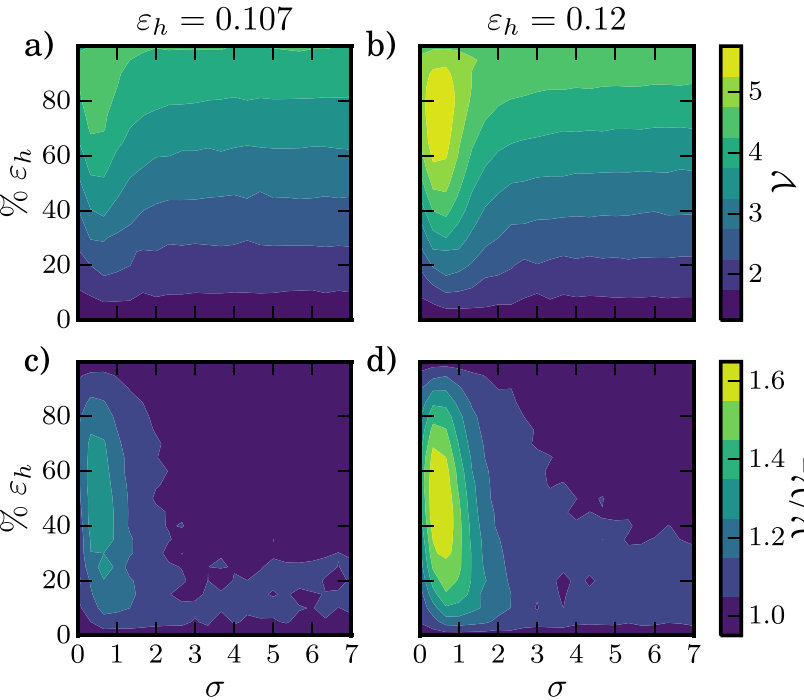

d)

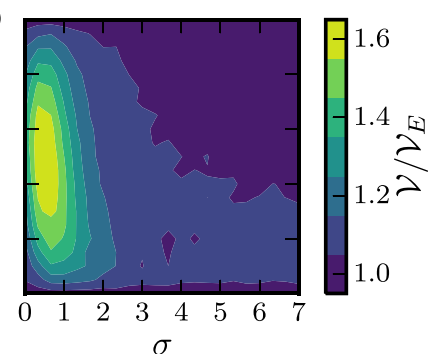

FIG. 6. Heat maps of PS volatility $\mathcal{V}$ in simulations of random patches of excitability as in Fig. 4, varying the percentage of the medium that has excitability $\varepsilon_{h}$ instead of $\varepsilon=0.08$ ( $y$ axis) and the spatial scale $\sigma$ ( $x$ axis) for two different $\varepsilon_{h}$. (a) and (b) PS volatility $\mathcal{V}$. (c) and (d) $\mathcal{V}$ normalized by the expected value $\mathcal{V}_{E}$, obtained by averaging the two homogeneous cases for $\varepsilon=0.08$ and $\varepsilon_{h}$ weighted by their respective fractional area. Each point represents the average of 20 simulations, the parameter grid step is 5\% for the area and $1 / 3$ for $\sigma$.

areas of PS are enclosed by excitability boundaries from all spatial directions. We therefore replaced the random patches by the simplest regular shape which fulfills this criterion, namely disks of lower excitability. We place these circular regions at random positions in the medium such that they do not overlap. Figure 7 shows snapshots of two such simulations with $\varepsilon_{h}=0.107$ inside the heterogeneities and different radii. The example in panels (a) and (c) shows the effect which we may expect based on the results in Sec. III B: PS cluster strongly inside the circular heterogeneities. However, the slightly larger radius in panels (b) and (d) shows clustering only within some of the disks and PS depletion outside the low-excitability zones is greatly enhanced, i.e., phase singularities seem to become trapped. This effect is also visible at the end of the $\mathcal{N}$ time series in Fig. 7(f) with only little fluctuation and a low average number of PS. Examining this type of heterogeneity more systematically (Fig. 8), we find that, indeed, the prominent emergent effect of the heterogeneities is an increase of $\mathcal{V}$ compared to the expected value $\mathcal{V}_{E}$, just like for random patches of lower excitability [e.g., around $R=2$ in Fig. 8(d)]. However, in rare instances, the effect can be reversed and the dynamics is stabilized, leading to lower than expected volatility, as visible for intermediate number of heterogeneities and $R=3.33$ in Fig. 8(c). Out of the simulated excitabilities, only $\varepsilon_{h}=0.107$ shows this substantial stabilization and decrease in $\mathcal{V}$. From Fig. $7(\mathrm{~d})$, it seems that the most effective trapping effect occurs inside heterogeneities that have a larger separation from neighboring lower-excitability regions (central and center-left disk).

\section{Circular heterogeneities on a regular grid}

In addition to the regular shape of the heterogeneities themselves, we therefore also enforce a certain distance by a)

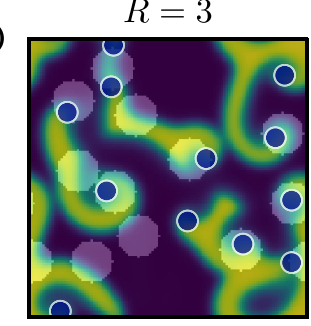

c)

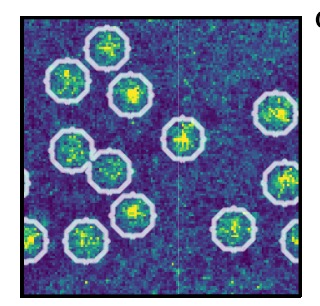

b)

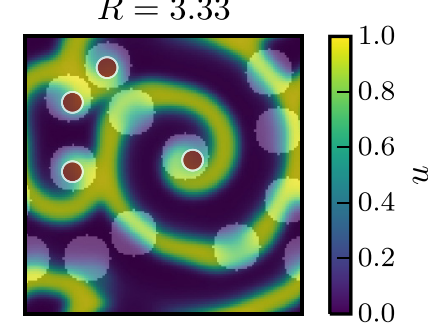

d)
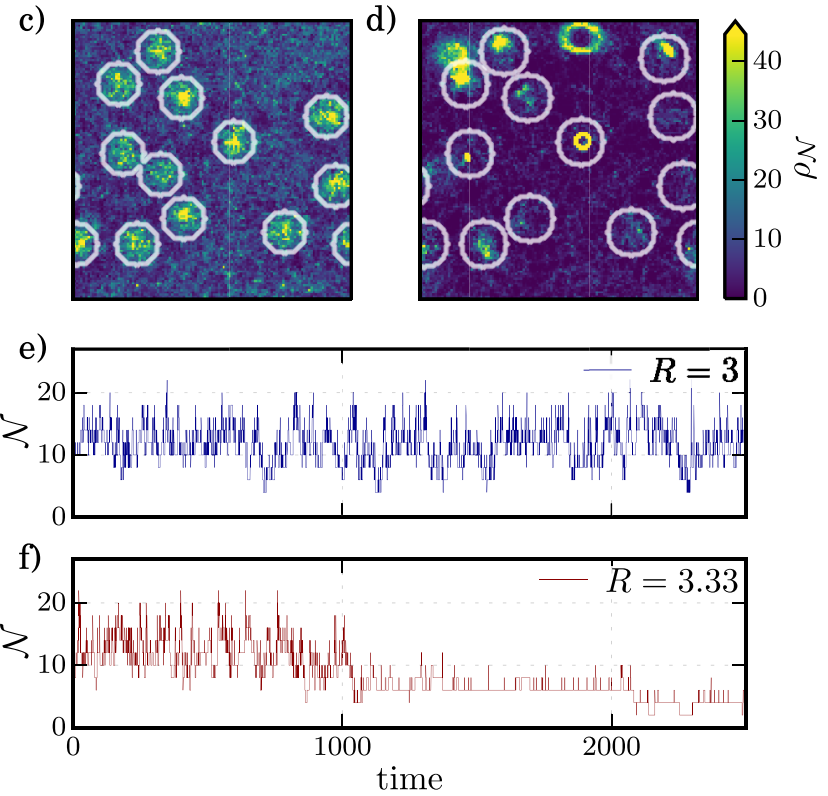

FIG. 7. Two simulations each containing 12 nonoverlapping circular regions in random locations with a lower excitability of $\varepsilon_{h}=0.107$ for two different radii. (a) and (b) Snapshots at $t=7500$ with PS marked by circles, the lighter areas correspond to lower excitability. (c) and (d) PS distribution per simulation domain integrated between $t=5000$ and $t=10000$ (excitability boundaries indicated by lines). Values of $\rho_{\mathcal{N}} \gtrsim 40$ are all represented by the same (yellow) color, indicated by the upward pointing colorbar. (e) and (f) Temporal development of $\mathcal{N}$ before the snapshot. The segments have a volatility of $\mathcal{V}=2.1$ and $\mathcal{V}=0.98$.

placing them on a regular grid. Two examples for heterogeneities with $\varepsilon_{h}=0.12$ on a $4 \times 4$ grid are depicted in Fig. 9, with the right column $(R=3.67)$ showing a case of complete stabilization. We confirmed this striking effect via the calculation of the Lyapunov dimension $D_{L}$, which, in contrast to $\mathcal{V}$, is independent of the detection of phase singularities, showing that these dynamics are indeed characterized by stable spiral waves.

We subsequently scanned this spatial configuration for three different $\varepsilon_{h}$ and different radii of the heterogeneities, and extracted the Kaplan-Yorke dimension $D_{L}$, the volatility $\mathcal{V}$ as well as the average $\mathcal{N}$ and lifespan statistics (Fig. 10). For $\varepsilon_{h}=0.093$, all measures of complexity stay close to their expected value and no stabilization occurs. For larger reductions in excitability and above a radius of $R=3$, however, $\mathcal{V}$ is significantly reduced. Interestingly, $D_{L}$ does not only confirm the striking stabilization effect within this range of radii range, but also shows an interesting behavior below $R=3$ : contrary to the expected value $D_{L, E}$, the Kaplan-Yorke dimension does not increase towards higher radii. Only after stabilization is lost towards larger radii does $D_{L}$ increase towards the expected value. While $\mathcal{N}$ tends to be lower in 


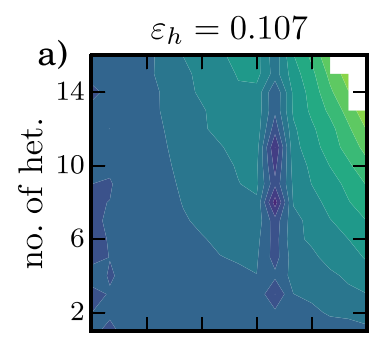

b)
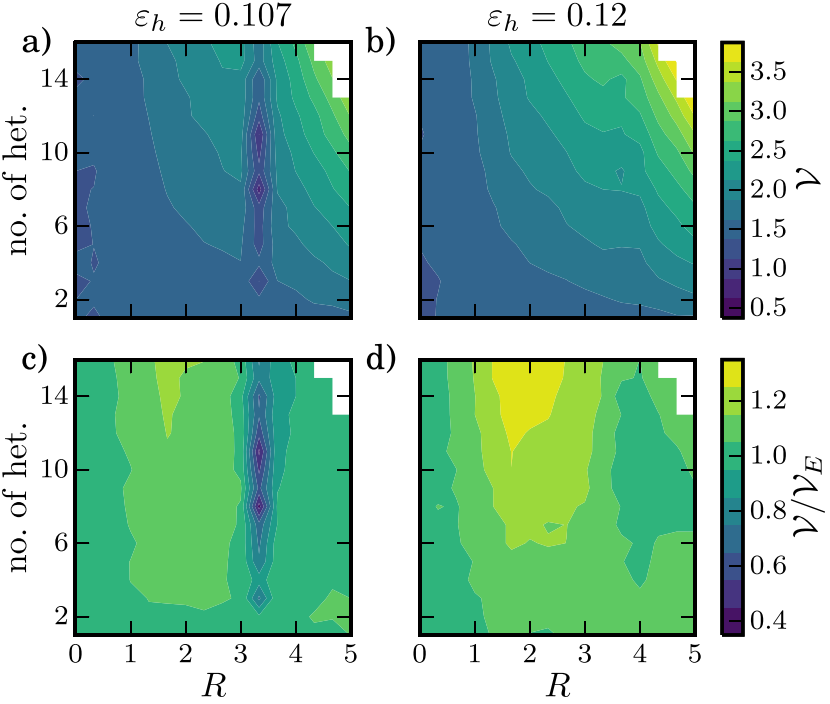

d)

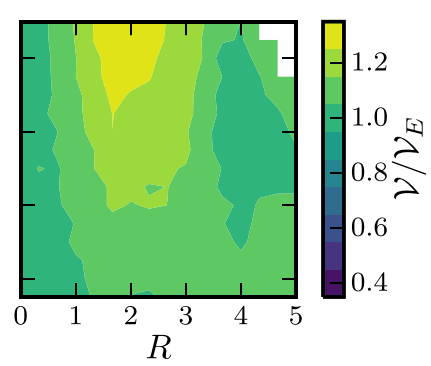

FIG. 8. PS volatility $\mathcal{V}$ as a function of the number of heterogeneities and their radius $R$ for simulations with randomly placed circular heterogeneities for two different $\varepsilon_{h}$. (a) and (b) PS volatility $\mathcal{V}$. (c) and (d) $\mathcal{V}$ normalized by the expected value $\mathcal{V}_{E}$ when weighting the two homogeneous $\varepsilon$ cases by the fractional area. Each point is the mean of 20 simulations. The parameter grid step is $1 / 3$ for $R$. Note that for the case of large and many circles, random placement becomes unlikely to succeed without overlap, so that a small area in the upper right corner is not sampled.

the stabilization region as well, the number of stable spiral cores is highly variable and can, in many cases, be just as high as in the homogeneous system, as indicated by the large error bars. It is important to note that stabilization usually occurs only after long transients, so some systems are in a fully stable state at the end of the simulation, while others are not. This is also visible in the lifespan statistics, which are shown for $\varepsilon_{h}=0.107$ in Fig. 10(d). For the stabilizing radii, the increase in lifespan is dramatic and standard deviations across simulations are large compared to radii that do not lead to stabilization.

In contrast to $D_{L}$, the volatility $\mathcal{V}$ shows a slight increase below $R=3$ compared to its expected value $\mathcal{V}_{E}$. This means that, while it appears generally possible to correlate large variations in $\mathcal{V}$ with changes in the complexity of the system, the analogy between the attractor dimension $D_{L}$ and $\mathcal{V}$ is certainly not perfect and care should be taken when trying to interpret small changes.

As the radius of the heterogeneity seems to be a critical parameter for stabilization to occur, we also extracted the power spectra for the case of $\varepsilon_{h}=0.107$ on a regular grid (Fig. 11) for different radii. For the same value of $\varepsilon_{h}$, stabilization even occurred for randomly placed heterogeneities for $R=3.33$ as discussed in Sec. III C (cf. Fig. 8), so one might suspect that certain properties of the trapped spiral waves enable stabilization even with large "gaps" in between the disks of lower excitability. For $R=3.33$ and $R=3.67$, the spectrum in Fig. 11 has clear peaks due to the stabilization, while spatio-temporal chaos for $R=3$ is characterized by a broad frequency spectrum similar to the homogeneous case. The frequency of the stabilized dynamics decreases with increasing $R$, which is intuitive at first since a larger fraction of the medium has an excitability which also yields a a)

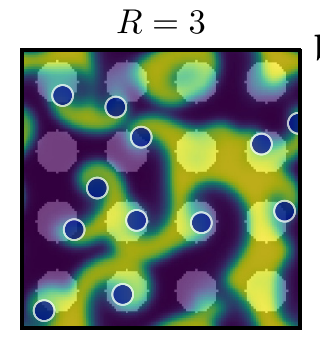

c)
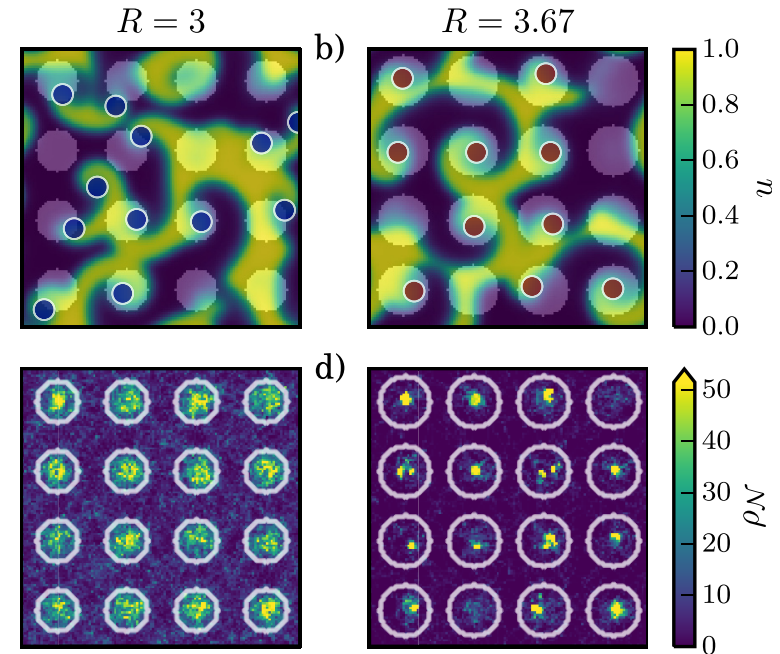

d)

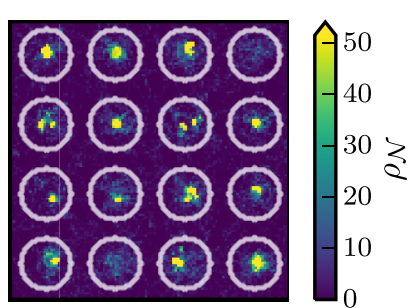

e)
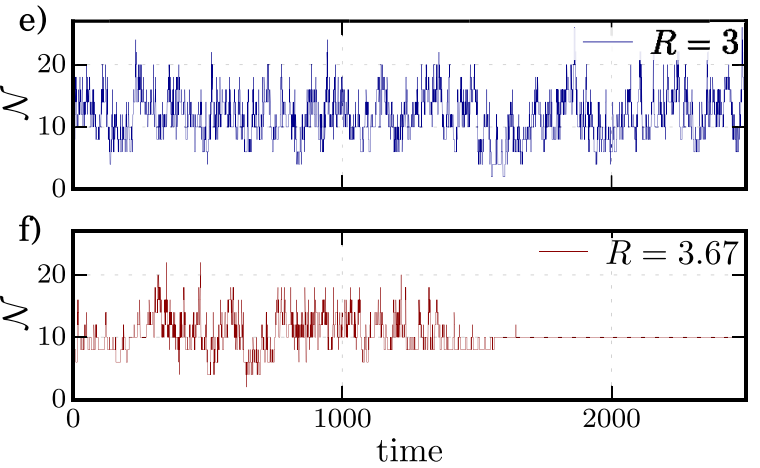

FIG. 9. Two simulations with 16 disks of lower excitability $\left(\varepsilon_{h}=0.12\right)$ placed on a regular $4 \times 4$ grid with different radii $R$. (a) and (b) Snapshots at $t=7500$ with PS marked as circles, the lighter areas correspond to lower excitability. (c) and (d) PS density per simulation domain calculated from $t=5000$ to $t=10000$ (excitability boundaries indicated by lines). Values of $\rho_{\mathcal{N}} \gtrsim 50$ are all represented by the same (yellow) color, indicated by the upward pointing colorbar. (e) and (f) Temporal development of $\mathcal{N}$ before the snapshot. The segments have a volatility of $\mathcal{V}=2.5$ and $\mathcal{V}=1.0$.

lower dominant frequency in the homogeneous case (Fig. 3). However, it is important to bear in mind that, in the stabilized state, spiral cores are trapped inside the lower excitability regions [Fig. 9(b)], which means that frequency of these spirals is still influenced by the surrounding environment. In this light, it is not surprising that the size of the heterogeneity might also impact the ability of waves emitted from trapped spiral cores to penetrate the rest of the medium and bridge large gaps between heterogeneities, as seen for $R=3.33$ in Fig. 8(c).

\section{E. Varying the separation between heterogeneities}

To probe this ability directly, we modified the regular grid scenario and varied the distance in between the diskshaped heterogeneities for a few selected radii. More specifically, we define the "separation" as the distance between the boundaries of neighboring heterogeneities. On the regular grid considered here with lattice parameter $d$, the separation therefore equals $d-2 R$. In contrast to all previous simulations, the medium size therefore changes for varying separation.

Figures 12 and 13 show the results for these scans for heterogeneities on a $4 \times 4$ grid with $\varepsilon_{h}=0.107$ and $\varepsilon_{h}=0.12$, 


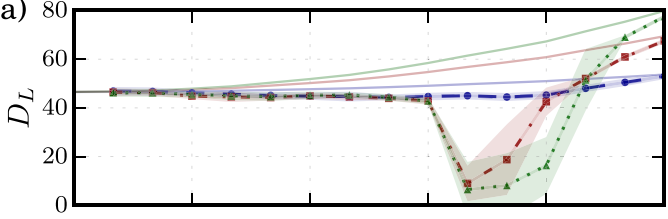

b)
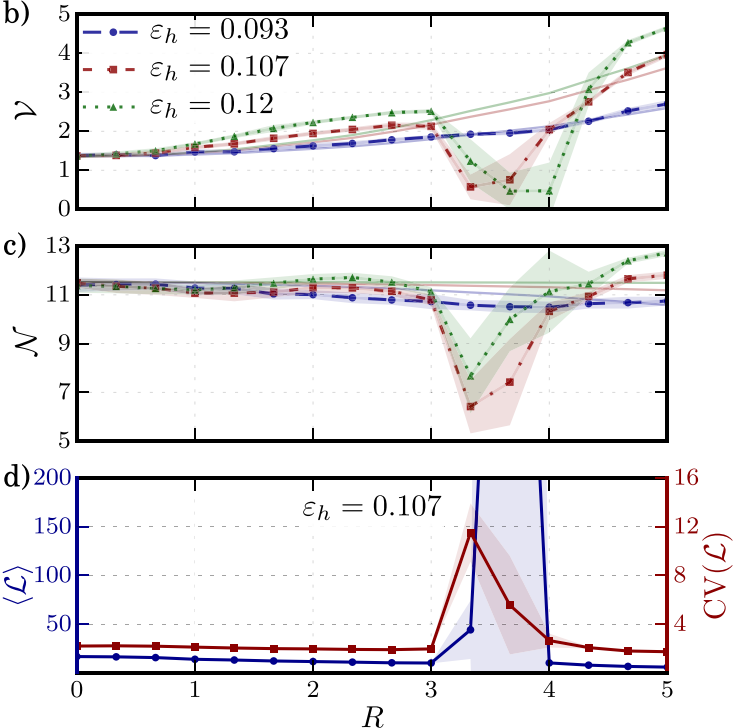

FIG. 10. Lyapunov dimension $D_{L}$, PS volatility $\mathcal{V}$, number of phase singularities $\mathcal{N}$, and lifespan statistics for circular heterogeneities placed on a $4 \times 4$ grid for varying radius $R$. (a) Mean $D_{L}$ of 20 runs each averaging from $t=8500$ to $t=10000$. The shaded areas indicate standard deviation across simulations. (b) and (c) Mean $\mathcal{V}$ and $\mathcal{N}$ of the same 20 runs, each averaging from $t=5000$ to $t=10000$. The thin solid lines in (a), (b), and (c) indicate expected $D_{L E}, \mathcal{V}_{E}$ and $\mathcal{N}_{E}$ when weighting the homogeneous results for $\varepsilon=$ 0.08 and $\varepsilon_{h}$ by the fractional area. (d) $\langle\mathcal{L}\rangle$ and $\operatorname{CV}(\mathcal{L})$ for one selected value of $\varepsilon_{h}=0.107$. The shaded areas indicate standard deviation across the same 20 simulations as in the other panels.

respectively. In both cases, stabilization occurs in a finite window of separations for most radii and the impact on $\mathcal{N}$ itself is relatively weak compared to the reduction in $\mathcal{V}$. While a too small separation between heterogeneities brings the system closer to the case of random (not well separated)
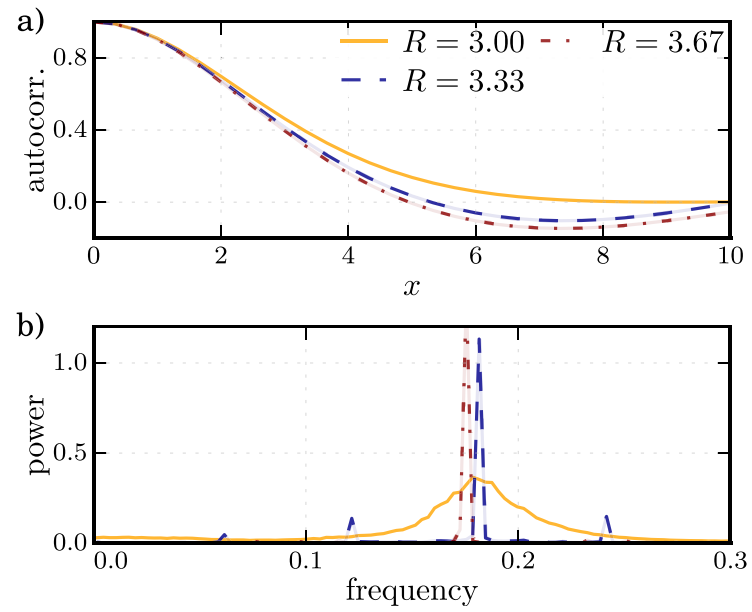

FIG. 11. (a) Spatial autocorrelation function for a medium with circular heterogeneities placed on a regular $4 \times 4$ grid for $\varepsilon_{h}=0.107$ and different radii. (b) Corresponding power spectra.

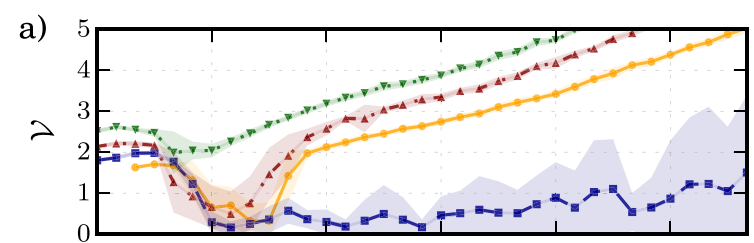

b)
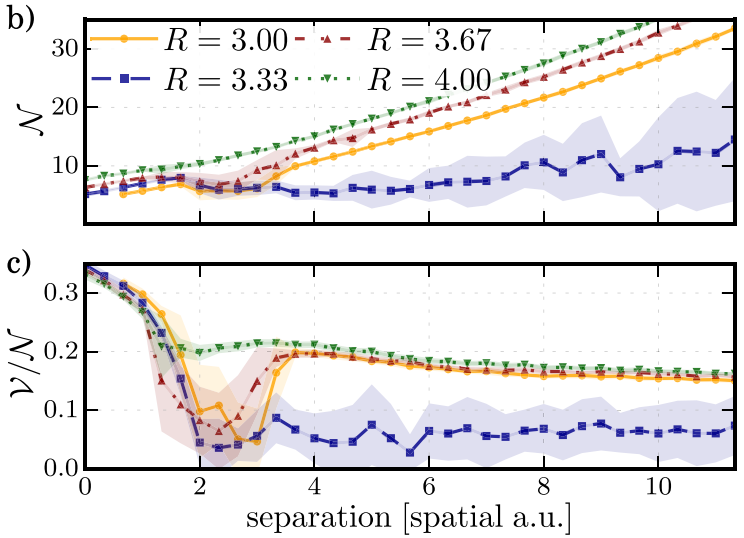

FIG. 12. PS volatility $\mathcal{V}$, the number of phase singularities $\mathcal{N}$, and their ratio for circular heterogeneities on a $4 \times 4$ grid with $\varepsilon_{h}=0.107$ and varying separation between the heterogeneities. Each value is based on the mean of 20 runs each averaging 5000 time units after a 5000 time unit transient. The shaded areas indicate the standard deviation across simulations.

heterogeneities as considered in Fig. 4, too large separations leave a large area in between the lower-excitability regions in which spatio-temporal chaos may survive. Figures 12 and 13 also show that, in cases where there is a significant reduction of $\mathcal{V}$ within a window of separations, these windows line up relatively well across different parameters. This means that separation between heterogeneities is a more decisive indicator for emergent behavior than the lattice parameter (i.e., the

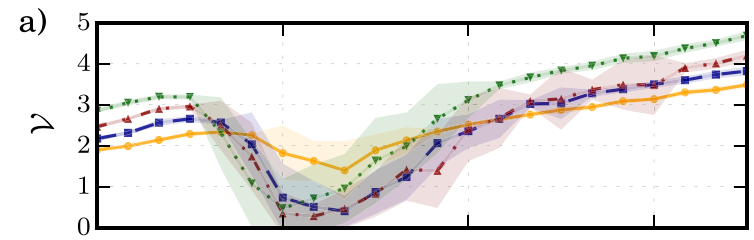

b)
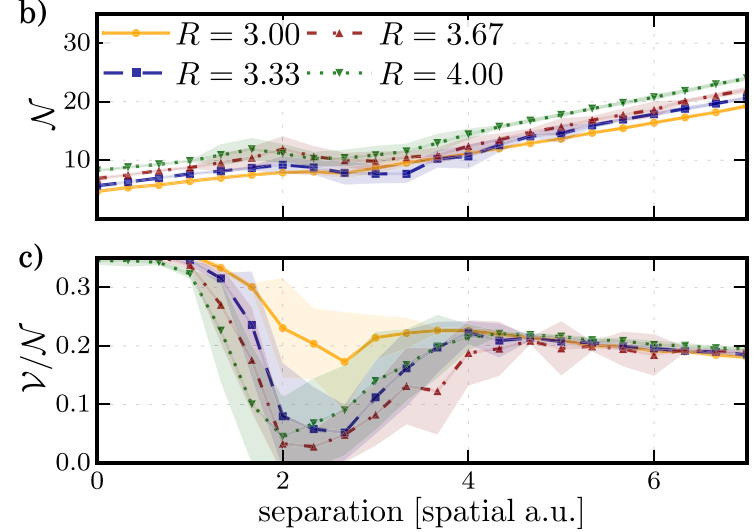

FIG. 13. PS volatility $\mathcal{V}$, the number of phase singularities $\mathcal{N}$, and their ratio for circular heterogeneities on a $4 \times 4$ grid with $\varepsilon_{h}=0.12$ and varying separation between the heterogeneities. Each value is based on the mean of 20 runs each averaging 5000 time units after a 5000 time unit transient. The shaded areas indicate the standard deviation across simulations. 
distance between the centers of the lower-excitability disks). As a function of the lattice parameter, each curve would be shifted by $2 R$ to the right, causing substantial misalignment between stability windows. The normalized volatility $\mathcal{V} / \mathcal{N}$ shown in panels (c) in both figures indicates that the slightly different absolute numbers for $\mathcal{V}$ outside the region of stabilization are mostly due to different absolute numbers of phase singularities: The curves of $\mathcal{V} / \mathcal{N}$ for different disk radii collapse almost perfectly for large separations, meaning that, in this regime, the size of the heterogeneity only changes the absolute numbers of phase singularities but not their intrinsic dynamics. In addition, this intrinsic volatility "per phase singularity" decreases towards larger separation, which is consistent with the fact that a larger and larger portion of the medium has the parameter value $\varepsilon=0.08$, which yields lower volatility (the apparent increase in panels (a) and (b) of both figures is merely due to the increase in medium size).

A notable deviation from the general pattern is the case of $R=3.33$ for $\varepsilon_{h}=0.107$ in Fig. 12, where strong stabilization occurs even for extremely large separations. This is similar to the findings for the randomly placed circles in Fig. 8, where only this case yielded stabilized dynamics. From the spectra shown in Fig. 11, one might suspect that the faster frequency for the case $R=3.33$ could allow waves emitted from trapped spiral waves to take over the area in between heterogeneities more easily. To examine this possibility, we extracted local periods at each point in the medium for representative simulations by detecting the time points at which the fast variable crosses the threshold $u^{*}=0.65$ and calculating intervals between consecutive crossing time points. We then averaged the past 6 periods at each point to obtain a measure for the local instantaneous period, while averaging out possible higher order rhythms (resulting, e.g., from Doppler shifts due to meandering). In this way, the transient dynamics that lead to stabilization can be visualized (Fig. 14). Indeed, for large separation and the "special" radius $R=3.33$ in panel (a), an area with homogeneous period emerges from a trapped spiral wave (marked by red star) and gradually takes over the medium. A similar mechanism does not seem to apply to the slightly larger radius $R=3.67$ in panel (b) which does not support stabilization for large separations according to Fig. 12, and homogeneous period areas around trapped spiral waves only occur transiently. In contrast, as depicted in panel (c), decreasing the separation for this radius makes stabilization possible and the transient looks similar to that in panel (a). It seems tempting to attribute the ability to take over the interstitial area to the shorter period of the stabilized waves in the case $\varepsilon=0.107$ and $R=3.33$ compared to $\varepsilon=$ 0.107 and $R=3.67$ [cf. panels (a) and (c)]. However, when we compare the period of the stabilized dynamics with that of the transient spatio-temporal chaos before stabilization, this mechanism is not unambiguously supported, as shown in Fig. 15: While the period for stabilized waves seems to be largely independent of the separation in the case in panel (c) where both large and small separations stabilize, it increases continuously with increasing radius $(\mathrm{b}) \rightarrow$ (c) $\rightarrow$ (d). The period analysis therefore fails to explain why the even faster period at $\varepsilon_{h}=0.107$ and $R=3$ cannot drive away interstitial activity and does not stabilize for larger separations.
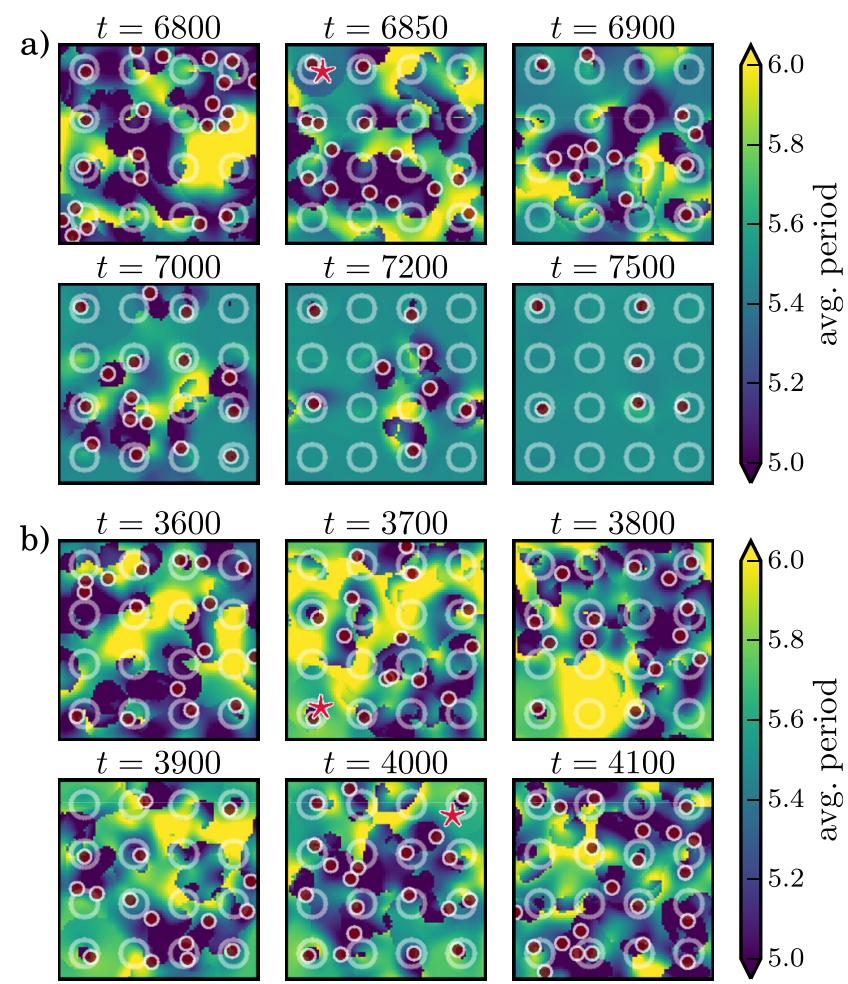
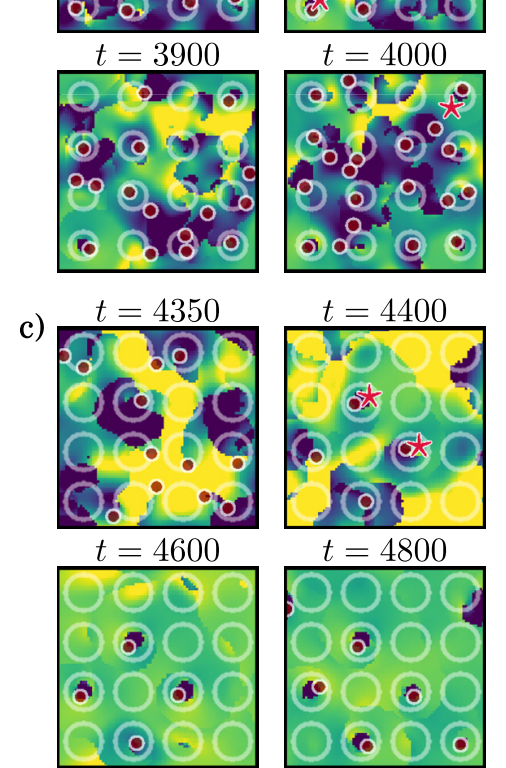

$t=4450$

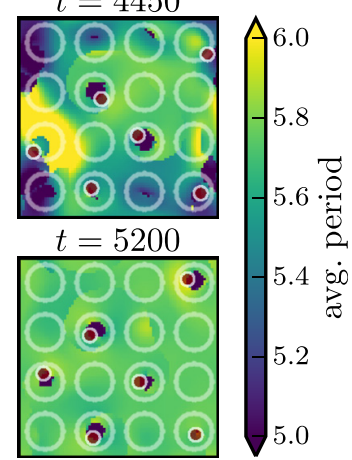

FIG. 14. Time evolution of instantaneous period in three systems during the stabilization process. The color indicates the average over the previous six previous periods detected from crossing the threshold $u^{*}=0.65$. The small circles mark the positions of the phase singularities. In all panels, the upward and downward pointing colorbars indicate that values above (below) the indicated maximum (minimum) are assigned the same color as the maximum (minimum) to enhance contrast within the range of interest. The stars indicate the heterogeneities with trapped spiral from which areas of homogeneous periods seem to emerge. (a) For $\varepsilon_{h}=0.107$ and $R=3.33$ and a separation of 6. (b) For $\varepsilon_{h}=0.107$ and $R=3.67$ and a separation of 6. (c) For $\varepsilon_{h}=0.12$ and $R=3.67$ and a separation of 2.67 .

\section{DISCUSSION}

In this study, we have investigated the emergent effects of heterogeneity on the complexity of spatio-temporal chaos in excitable media. While it was already known that heterogeneity can affect the stability and dynamics of isolated spiral waves, ${ }^{27,28,31-33,35}$ our results show that even for sustained spiral wave breakup conditions, heterogeneities in excitability can strongly influence the dynamics. According to our simulations, sharp local reductions in excitability can have strikingly different effects depending on their spatial arrangement in the medium. Even for the limited parameters 

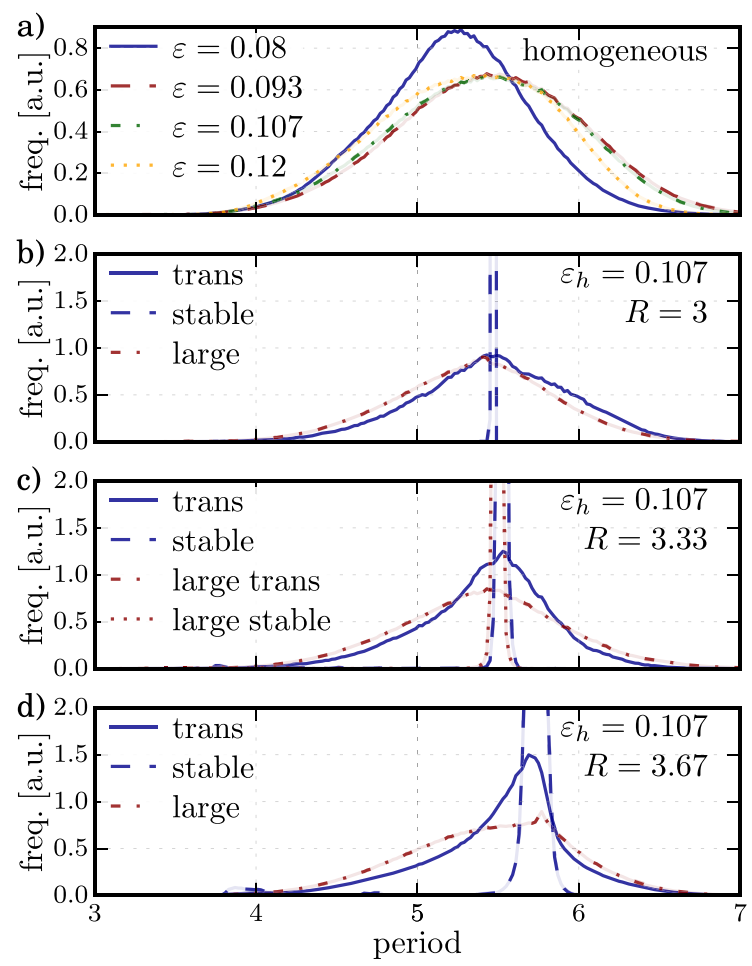

FIG. 15. Histograms over averages of six periods in various scenarios "large" indicates a separation of 6 instead of 2.67 between the heterogeneities. "trans" refers to the transient dynamics at the beginning of a simulation, whereas data for "stable" is extracted after the dynamics is fully stabilized. Since the stabilization occurs after a transient time period of random length ( $c f$. Fig. 14), the specific time periods used to calculate period histograms were chosen manually in each case. (a) The homogeneous case for comparison. (b)-(d) Each with $\varepsilon_{h}=0.107$ and increasing radius. Only the radius of $R=3.33$ in (c) shows a stabilization for a large separation of 6 , while all cases may stabilize for a separation of 2.67 .

investigated here, a zoo of different effects were discovered which range from an increase in turbulence to a complete stabilization of the spiral wave activity. We detected emergent behavior by comparing different complexity measures to the naive assumption that complexity in different parts of the medium is dictated by the local value of the excitability and hence the total complexity can be predicted by averaging of the results from the homogeneous scenarios (Fig. 2) weighted by their respective fractional areas in the medium. For example, small scale random patches of lower excitability can almost double the PS volatility $\mathcal{V}$ with respect to this expectation to values that are outside the range of observed values for any of the homogeneous parameters (cf. Figs. 2 and 6). Conversely, circular regions of lower excitability, placed in random positions as well as on a regular grid, can stabilize spiral waves up to the point when spatio-temporal chaos is completely suppressed (see Figs. 7 and 9). This effect is particularly surprising considering that all local parameters contributing to these stabilized cases lead to chaotic dynamics in a homogeneous setting. This is in contrast to, for example, a study by Shajahan et al., where qualitatively different parameters that do or do not lead to breakup were combined by introducing a single isolated heterogeneity. ${ }^{20}$ Moreover, media with a homogeneously lower excitability show turbulence with an even higher attractor dimension $D_{L}$ and PS volatility $\mathcal{V}$ (Fig. 2), whereas the same parameters limited to small regions below a certain critical size do not increase $D_{L}$ (Fig. 10).

While the exact mechanism of the stabilization is still unclear, our results provide evidence pointing to several different factors that could play a role. One very generic mechanism observed in almost all scenarios investigated in this study is the tendency of reduced excitability regions to attract phase singularities, thereby extending the mechanism of spiral wave drift towards regions of lower excitability and lower frequency ${ }^{31-33}$ to the case of spatio-temporal chaos, where "lower frequency" has to be replaced by "lower dominant frequency" (cf. Fig. 3). This observation parallels the pinning effect to regions of longer APD in a model of atrial fibrillation with periodically varying excitability. ${ }^{34}$ Since the phase singularities in our study localize mostly close the interface between regions of different excitability (see Fig. 5), this could be related to the mechanism theoretically investigated by Biktasheva et al. (but for chaotic parameters), according to which spiral waves are attracted and can drift along the boundaries of localized heterogeneities. ${ }^{27,29}$ Since this drift mechanism is based on response functions which are localized at the core of spiral waves, one might suspect that the (de-)stabilization dynamics observed here are mainly due to direct interaction of heterogeneities with the organizing centers of spiral waves. However, it is unclear how these results can be transferred to the case of spatiotemporal chaos.

Interestingly, the resonant radius that leads to entrapment of spiral waves in circular heterogeneities (see Figs. 10, 12 and 13) is on the same order as the width of the region of increased PS density close to the stepwise heterogeneity in Fig. 5. This radius might therefore represent an optimal trade-off: large enough to enclose the whole region of increased PS density attracted by the step in excitability, and small enough such that there is no room for the spiral wave to destabilize, as it would usually do at these local parameters. An interesting direction for future research would be to try to compute the stability properties (and/or response functions) of a single stabilized spiral wave in the "resonant" heterogeneous parameter settings with circular heterogeneities, which should be possible due to the rotational symmetry of the problem. This way, it could be possible to uncover which of the characteristic length scales of spiral waves (wave length, core size, etc.) is responsible for the resonant effect, and whether such a local determinant exists at all. Potentially, this analysis could also shed some more light on the unknown factors which determine the properties of waves emitted outwards by stabilized spirals that presumably determine to what extent they can compete with chaotic activity present in the medium. Our results indicate that too large deviations in either direction from the optimum size lead to a loss of the stabilizing effect: For larger scale heterogeneities, locations deep inside do not feel the boundary and therefore chaos develops as in the homogeneous medium. Smaller heterogeneities, on the other hand, do not contain the full potential trapping region of Fig. 5 and can even enhance PS volatility by transiently attracting phase 
singularities without trapping them (Fig. 4), apparently leading to more turbulent dynamics.

Other factors that determine the strength of the stabilizing effect are the number of heterogeneities and the size of the area in between them, whose investigation both result in the same conclusion: the density of heterogeneities must be large enough such that each PS has a high probability of simultaneously interacting with heterogeneity. Too large separation between heterogeneities leaves too much room such that chaos can survive. As pointed out above for the radius, the optimal separation between heterogeneities (Figs. 12 and 13) seems to have the same length scale as the interface effect shown in Fig. 5, meaning that depletion zones of PS between heterogeneities might be equally important for stabilizing the dynamics. This is in stark contrast to situations where a single stabilized rotor can drive away any remaining turbulent activity due to a higher rotation frequency. ${ }^{44}$ Our frequency analysis shows that the frequency of spiral waves is generally decreased in media of lower excitability (Fig. 3), leading to the inability of trapped spiral waves to do the same in most cases. However, if stabilized spirals happen to have this ability as seen in Fig. 14(a), they can bridge large separations between heterogeneities (Fig. 12) and also tolerate more random arrangements of lowerexcitability regions (Fig. 8), although it is currently not clear what determines this ability.

There seems to be a general relation between the degree of randomness and the stabilizing effect of the lowerexcitability regions: From random patches which always increase turbulence (Fig. 6) to randomly placed circular regions with stabilization occurring only very rarely (Fig. 7) to circular regions on a grid with at most mild increases in turbulence and stabilization in larger parameter regimes (Figs. 10, 12, and 13). This suggests that in realistic settings without perfect shape and order, increases in turbulence are the more likely result of heterogeneity, although this expectation should be tested with physiologically more faithful models for the local dynamics. On a more abstract level, the role of randomness reveals an interesting interplay between the structure and dynamics during spatio-temporal chaos: while heterogeneity in general, and specifically that associated with lower excitability, could be expected to increase turbulence, it also imposes a certain spatial structure that spiral waves can interact with. As our results show, the ordering effect of this spatial structure can outweigh the turbulenceincreasing tendency which is apparent for more random arrangements of heterogeneities (Fig. 4). Other types of heterogeneity that have a strong influence on spiral wave dynamics, such as a decrease in intercellular coupling or impermeable inclusions, have been shown to be able to stabilize or even lead to complete spontaneous termination of reentrant activity in two and three dimensions. ${ }^{22,44-46}$ The effects shown in our study are of a different quality, in that activity can still enter the lower excitability regions unhamperedly and the parameters inside the heterogeneity do not in themselves stabilize or terminate the activity (except for the tendency for PS to cluster at the border, see above). One consequence of this is that, as mentioned above, stabilization does not occur for too large heterogeneities, whereas, for example, for impermeable inclusions, effects may also start at a critical size but then persist towards large radii. ${ }^{45}$

In the context of cardiac arrhythmias, our results contribute to the mounting evidence that heterogeneity of tissue properties can have a profound impact on cardiac arrhythmias. While Bishop et al. found that the natural regional variations in electrical tissue properties in the ventricle play a critical role during arrhythmia onset but do not significantly influence the statistical properties of fully developed arrhythmias, ${ }^{47}$ the emergent effects discovered here prove that local differences in excitability due to, for example, remodeling, fibrosis or ischemic zones, have the potential to change the characteristics of fibrillation itself. Considering the intense research efforts to link certain local dynamics, e.g., genetic abnormalities, contributions of different trans-membrane currents, cell-to-cell coupling, etc., to the arrhythmogenicity of the cardiac substrate, the results presented in this paper add an additional aspect to the widely known fact that the dependence of spatio-temporal tissue dynamics-i.e., whether they support functional reentry, whether reentry is stable, etc. - on local parameters is rather non-trivial. Even if the relation between homogeneous local parameters and spatio-temporal dynamics was completely understood, the emergent effects discovered here mean that there could, for example, be situations when all locally determined tissue properties indicate susceptibility to fibrillation, but a specific spatial distribution of these parameters prevents spatiotemporal chaos from actually occurring. This emphasizes the need for faithful spatially resolved numerical models for concrete applications.

As intervention protocols become more sophisticated and more and more tailored to the specific type of the arrhythmia, ${ }^{48-52}$ being able to assess the complexity of the arrhythmia and understand its dependence on pathological heterogeneities might also become more important in order to fine-tune the termination strategy. An interesting question that arises in this context is what turbulence measures like the attractor dimension $D_{L}$ and the PS volatility $\mathcal{V}$ actually mean for termination efforts: While, intuitively, lower complexity certainly means "simpler," artificially stabilized arrhythmias might actually be harder to terminate, as has been shown for waves pinned to non-conducting heterogeneities, ${ }^{53-57}$ compared to very turbulent spiral chaos, where phase singularities fluctuate strongly. In the latter case, one could imagine that larger fluctuations also increase the chances to influence the dynamics with weak stimuli that cause phase singularities to annihilate or drift into boundaries. ${ }^{58,59}$ Therefore, investigating how termination success correlates with these turbulence indicators and the development of alternative metrics is also an interesting direction for future research.

\section{ACKNOWLEDGMENTS}

We acknowledge support from the German Federal Ministry of Education and Research (BMBF) (Project No. FKZ 031A147, GO-Bio), the German Center for Cardiovascular Research (DZHK), Partner Site Göttingen, and the German Research Foundation through SFB 1002 
Modulatory Units in Heart Failure (project C03). P.B. acknowledges support from the HFSP fellowship LT000840/ 2014-C.

${ }^{1} \mathrm{G}$. Ertl, "Oscillatory kinetics and spatio-temporal self-organization in reactions at solid surfaces," Science 254, 1750-1755 (1991).

${ }^{2}$ W. Jahnke, W. Skaggs, and A. Winfree, "Chemical vortex dynamics in the Belousov-Zhabotinsky reaction and in the 2-variable Oregonator model," J. Phys. Chem. 93, 740-749 (1989).

${ }^{3}$ B. Vasiev, P. Hogeweg, and A. Panfilov, "Simulation of Dictyostelium Discoideum aggregation via reaction-diffusion model," Phys. Rev. Lett. 73, 3173-3176 (1994).

${ }^{4}$ J. Davidenko, A. Pertsov, R. Salomonsz, W. Baxter, and J. Jalife, "Stationary and drifting spiral waves of excitation in isolated cardiac muscle," Nature 355, 349-351 (1992).

${ }^{5}$ A. Pertsov, J. Davidenko, R. Salomonsz, W. Baxter, and J. Jalife, "Spiral waves of excitation underlie reentrant activity in isolated cardiac muscle,' Circ. Res. 72, 631-650 (1993).

${ }^{6}$ R. Gray, A. Pertsov, and J. Jalife, "Spatial and temporal organization during cardiac fibrillation," Nature 392, 75-78 (1998).

${ }^{7}$ F. Witkowski, L. Leon, P. Penkoske, W. Giles, M. Spano, W. Ditto, and A. Winfree, "Spatiotemporal evolution of ventricular fibrillation," Nature 392, 78-82 (1998).

${ }^{8}$ R. H. Clayton, O. Bernus, E. M. Cherry, H. Dierckx, F. H. Fenton, L. Mirabella, A. V. Panfilov, F. B. Sachse, G. Seemann, and H. Zhang, "Models of cardiac tissue electrophysiology: Progress, challenges and open questions," Prog. Biophys. Mol. Biol. 104, 22-48 (2011).

${ }^{9}$ S. Alonso, M. Bär, and B. Echebarria, "Nonlinear physics of electrical wave propagation in the heart: A review," Rep. Prog. Phys. 79, 096601 (2016).

${ }^{10} \mathrm{C}$. Antzelevitch and J. Fish, "Electrical heterogeneity within the ventricular wall,” Basic Res. Cardiol. 96, 517-527 (2001).

${ }^{11} \mathrm{P}$. Kohl, "Cardiac cellular heterogeneity and remodelling," Cardiovasc. Res. 64, 195-197 (2004).

${ }^{12}$ E. M. Cherry and S. J. Evans, "Properties of two human atrial cell models in tissue: Restitution, memory, propagation, and reentry," J. Theor. Biol. 254, 674-690 (2008).

${ }^{13}$ E. Carmeliet, "Cardiac ionic currents and acute ischemia: From channels to arrhythmias," Physiol. Rev. 79, 917-1017 (1999); available at https:// www.ncbi.nlm.nih.gov/pubmed/10390520.

${ }^{14}$ S. Nattel, "Experimental evidence for proarrhythmic mechanisms of antiarrhythmic drugs," Cardiovasc. Res. 37, 567-577 (1998).

${ }^{15}$ P. Kohl, P. Camelliti, F. L. Burton, and G. L. Smith, "Electrical coupling of fibroblasts and myocytes: Relevance for cardiac propagation," J. Electrocardiol. 38, 45-50 (2005).

${ }^{16}$ M. Miragoli, G. Gaudesius, and S. Rohr, "Electrotonic modulation of cardiac impulse conduction by myofibroblasts," Circ. Res. 98, 801 (2006).

${ }^{17}$ M. S. Spach, "Mounting evidence that fibrosis generates a major mechanism for atrial fibrillation," Circ. Res. 101, 743-745 (2007).

${ }^{18} \mathrm{~S}$. Rohr, "Myofibroblasts in diseased hearts: New players in cardiac arrhythmias?," Heart Rhythm 6, 848-856 (2009).

${ }^{19}$ I. V. Kazbanov, K. H. W. J. ten Tusscher, and A. V. Panfilov, "Effects of heterogeneous diffuse fibrosis on arrhythmia dynamics and mechanism," Sci. Rep. 6, 20835 (2016).

${ }^{20}$ T. K. Shajahan, A. R. Nayak, and R. Pandit, "Spiral-wave turbulence and its control in the presence of inhomogeneities in four mathematical models of cardiac tissue," PLoS One 4, e4738 (2009).

${ }^{21}$ V. Zykov, A. Krekhov, and E. Bodenschatz, "Fast propagation regions cause self-sustained reentry in excitable media," Proc. Natl. Acad. Sci. U.S.A. 114, 1281-1286 (2017)

${ }^{22}$ E. Boccia, U. Parlitz, and S. Luther, "Spontaneous termination of reentrant activity under myocardial acute ischemia: Role of cellular conductivity and its relation to ischemic heterogeneities," Commun. Nonlinear Sci. Numer. Simul. 48, 115-122 (2017).

${ }^{23}$ E. Boccia, S. Luther, and U. Parlitz, "Modelling far field pacing for terminating spiral waves pinned to ischaemic heterogeneities in cardiac tissue," Philos. Trans. R. Soc. A 375, 20160289 (2017).

${ }^{24} \mathrm{D}$. Barkley, "Linear stability analysis of rotating spiral waves in excitable media," Phys. Rev. Lett. 68, 2090-2093 (1992).

${ }^{25} \mathrm{D}$. Barkley, "Euclidean symmetry and the dynamics of rotating spiral waves," Phys. Rev. Lett. 72, 164-167 (1994).

${ }^{26}$ D. Allexandre and N. F. Otani, "Preventing alternans-induced spiral wave breakup in cardiac tissue: An ion-channel-based approach," Phys. Rev. E 70, 061903 (2004).
${ }^{27}$ I. V. Biktasheva, D. Barkley, V. N. Biktashev, and A. J. Foulkes, "Computation of the drift velocity of spiral waves using response functions," Phys. Rev. E 81, 066202 (2010).

${ }^{28}$ I. V. Biktasheva, A. V. Holden, and V. N. Biktashev, "Localization of response functions of spiral waves in the FitzHugh-Nagumo system," Int. J. Bifurcation Chaos 16, 1547-1555 (2006).

${ }^{29}$ V. G. Fast and A. M. Pertsov, "Drift of a vortex in the myocardium," Biofizika 35, 478-482 (1990).

${ }^{30} \mathrm{C}$. W. Zemlin and A. M. Pertsov, "Anchoring of drifting spiral and scroll waves to impermeable inclusions in excitable media," Phys. Rev. Lett. 109, 038303 (2012).

${ }^{31}$ A. V. Panfilov and B. Vasiev, "Vortex initiation in a heterogeneous excitable medium,” Physica D 49, 107-113 (1991).

${ }^{32}$ P. Sadeghi and H. H. Rotermund, "Gradient induced spiral drift in heterogeneous excitable media," Chaos 21, 013125 (2011).

${ }^{33}$ K. H. W. J. ten Tusscher and A. V. Panfilov, "Reentry in heterogeneous cardiac tissue described by the Luo-Rudy ventricular action potential model," Am. J. Physiol.: Heart Circ. Physiol. 284, H542-H548 (2003).

${ }^{34} \mathrm{P}$. Comtois and S. Nattel, "Impact of tissue geometry on simulated cholinergic atrial fibrillation: A modeling study," Chaos 21, 013108 (2011).

${ }^{35}$ S. Sridhar, S. Sinha, and A. V. Panfilov, "Anomalous drift of spiral waves in heterogeneous excitable media,” Phys. Rev. E 82, 051908 (2010).

${ }^{36} \mathrm{M}$. Bär and M. Eiswirth, "Turbulence due to spiral breakup in a continuous excitable medium,” Phys. Rev. E 48, R1635-R1637 (1993).

${ }^{37}$ M. C. Strain and H. S. Greenside, "Size-dependent transition to highdimensional chaotic dynamics in a two-dimensional excitable medium," Phys. Rev. Lett. 80, 2306 (1998).

${ }^{38} \mathrm{D}$. Barkley, "A model for fast computer simulation of waves in excitable media," Physica D 49, 61-70 (1991).

${ }^{39}$ M. Bär and M. Or-Guil, "Alternative scenarios of spiral breakup in a reaction-diffusion model with excitable and oscillatory dynamics," Phys. Rev. Lett. 82, 1160 (1999).

${ }^{40} \mathrm{P}$. Bittihn, "Complex structure and dynamics of the heart," Springer Theses (Springer International Publishing, 2015).

${ }^{41}$ G. Benettin, L. Galgani, A. Giorgilli, and J.-M. Strelcyn, "Lyapunov characteristic exponents for smooth dynamical systems and for Hamiltonian systems; A method for computing all of them. Part 1: Theory," Meccanica 15, 9-20 (1980)

${ }^{42}$ G. Benettin, L. Galgani, A. Giorgilli, and J.-M. Strelcyn, "Lyapunov characteristic exponents for smooth dynamical systems and for Hamiltonian systems; A method for computing all of them. Part 2: Numerical application," Meccanica 15, 21-30 (1980).

${ }^{43}$ A. Iyer and R. Gray, "An experimentalist's approach to accurate localization of phase singularities during reentry," Ann. Biomed. Eng. 29, 47-59 (2001).

${ }^{44}$ F. Spreckelsen, D. Hornung, O. Steinbock, U. Parlitz, and S. Luther, "Stabilization of three-dimensional scroll waves and suppression of spatiotemporal chaos by heterogeneities," Phys. Rev. E 92, 042920 (2015).

${ }^{45}$ F. Xie, Z. Qu, and A. Garfinkel, "Dynamics of reentry around a circular obstacle in cardiac tissue,” Phys. Rev. E 58, 6355 (1998).

${ }^{46} \mathrm{Z}$. Qu, "Critical mass hypothesis revisited: Role of dynamical wave stability in spontaneous termination of cardiac fibrillation," Am. J. Physiol.: Heart Circ. Physiol. 290, H255-H263 (2005).

${ }^{47}$ M. J. Bishop, E. J. Vigmond, and G. Plank, "The functional role of electrophysiological heterogeneity in the rabbit ventricle during rapid pacing and arrhythmias," Am. J. Physiol.: Heart Circ. Physiol. 304, H1240-H1252 (2013).

${ }^{48}$ I. Gussak, C. Antzelevitch, A. A. M. Wilde, P. A. Friedman, M. J. Ackerman, and W.-K. Shen, eds., Electrical Diseases of the Heart (Springer London, London, 2008).

${ }^{49}$ S. Luther, F. H. Fenton, B. G. Kornreich, A. Squires, P. Bittihn, D. Hornung, M. Zabel, J. Flanders, A. Gladuli, L. Campoy, E. M. Cherry, G. Luther, G. Hasenfuss, V. I. Krinsky, A. Pumir, R. F. Gilmour, and E. Bodenschatz, "Low-energy control of electrical turbulence in the heart," Nature 475, 235-239 (2011).

${ }^{50}$ W. Li, A. H. Janardhan, V. V. Fedorov, Q. Sha, R. B. Schuessler, and I. R. Efimov, "Low-energy multistage atrial defibrillation therapy terminates atrial fibrillation with less energy than a single shock," Circ.: Arrhythmia Electrophysiol. 4, 917-925 (2011).

${ }^{51}$ C. M. Ambrosi, C. M. Ripplinger, I. R. Efimov, and V. V. Fedorov, "Termination of sustained atrial flutter and fibrillation using low-voltage multiple-shock therapy," Heart Rhythm 8, 101-108 (2011).

${ }^{52}$ W.-J. Rappel, J. A. B. Zaman, and S. M. Narayan, "Mechanisms for the termination of atrial fibrillation by localized ablation," Circ.: Arrhythmia Electrophysiol. 8, 1325-1333 (2015). 
${ }^{53}$ S. Takagi, A. Pumir, D. Pazo, I. Efimov, V. P. Nikolski, and V. I. Krinsky, "Unpinning and removal of a rotating wave in cardiac muscle," Phys. Rev. Lett. 93, 058101 (2004).

${ }^{54}$ C. M. Ripplinger, V. I. Krinsky, V. P. Nikolski, and I. R. Efimov, "Mechanisms of unpinning and termination of ventricular tachycardia," Am. J. Physiol.: Heart Circ. Physiol. 291, H184-H192 (2006).

${ }^{55}$ P. Bittihn, G. Luther, E. Bodenschatz, V. I. Krinsky, U. Parlitz, and S. Luther, "Far field pacing supersedes anti-tachycardia pacing in a generic model of excitable media," New J. Phys. 10, 103012 (2008).

${ }^{56}$ A. Pumir, S. Sinha, S. Sridhar, M. Argentina, M. Hörning, S. Filippi, C. Cherubini, S. Luther, and V. I. Krinsky, "Wave-train-induced termination of weakly anchored vortices in excitable media," Phys. Rev. E 81, 010901 (2010).

${ }^{57}$ A. Isomura, M. Hörning, K. Agladze, and K. Yoshikawa, "Eliminating spiral waves pinned to an anatomical obstacle in cardiac myocytes by highfrequency stimuli," Phys. Rev. E 78, 066216 (2008).

${ }^{58}$ S. Morgan, I. Biktasheva, and V. Biktashev, "Control of scroll-wave turbulence using resonant perturbations," Phys. Rev. E 78, 046207 (2008).

${ }^{59}$ S. Alonso, F. Sagues, and A. Mikhailov, "Taming winfree turbulence of scroll waves in excitable media," Science 299, 1722-1725 (2003).

${ }^{60}$ T. Lilienkamp, J. Christoph, and U. Parlitz, "Features of chaotic transients in excitable media governed by spiral and scroll waves," Phys. Rev. Lett. 119, 054101 (2017) 


\subsection{Experimental Findings of Spatial Structuring}

In the previous manuscript, the spatial structuring of PS due to dynamical heterogeneity was discussed. Further, a stabilization was shown and linked to this structuring. Since the strong stabilization depends on specific parameters it cannot be easily prepared in an experimental setting. However, the general organization effects seen in Figure 5 of the manuscript may be found. Also, in the experimental setting dynamical heterogeneities arise and reorganization along boundaries can occur.

To show that spatial organization due to dynamical heterogeneity occurs in the experiment, I will show that two hypotheses appear to be true:

1. Spatial structure seen in the PS distribution increases with larger spatial heterogeneity in the dynamical properties.

2. The spatial distribution of PS correlates with the expected reorganization due to the spatial heterogeneity.

Showing both of these hypotheses to be true gives very good indications that the effects studied in the manuscript are relevant in the heart. Showing the second hypothesis means that PS structure and dynamical heterogeneity are linked. However, such a link could have a common underlying cause in heterogeneities such as the vasculature. Additionally showing the first hypothesis makes the argument much stronger. It confirms the same observations in the numerical model and, more importantly, a common underlying cause seems unlikely to show such a relationship.

To test both hypotheses the spatial PS density as well as the expected structuring due to dynamical heterogeneity needs to be quantified. The former is directly accessible from PS identification and tracking as introduced in Section 4.4 and this analysis gives the result shown in Figure 7.2 (a). To quantify the expected structure the dynamical heterogeneity needs to be quantified first. Two main observables are directly available to indicate this heterogeneity and were used in the previous chapters:

1. The action potential duration (APD) is affected by local properties of the heart. For example it may shorten due to ischemia. The APD and its calculation is defined in Section 4.3.3.

2. The triangularity index $(\Delta)$ - a measurement for the action potential shape may similarly indicate changes such as local ischemic conditions. During his Bachelorthesis, Benjamin Böbel recently found that the spatial structure of the APD can differ from that of the triangularity index. The definition and method for calculating the triangularity index is described in Section 4.3.4.

In the first attempt, I used the APD to map the dynamical heterogeneity of the heart. However, doing the analysis shown later in this chapter based on the APD does not give as compelling evidence. Thus, here the second method is used and the following analysis is based on the triangularity index map calculated at an $80 \%$ level. An example of such a triangularity index map can be found in Figure 7.2 (a). 


\section{Estimation of the PS Density}

In general, it is unlikely that different PS identification methods should give strongly differing results for the PS density. For example Liu et al. [88] compared two different PS identification methods in this regard and find a very similar structure. Here, the PS density is estimated with the same method for PS identification used throughout the thesis and detailed in Section 4.4.

The PS density can be found directly from the PS tracking data. However, the PS data has too few events considering the high spatial resolution of the cameras. Thus, it is necessary to smoothen the data using methods such as kernel density estimation. For the following analysis the PS density is thus spatially smoothed using a Gaussian function with $\sigma=3$. However, the results do not appear to be sensitive to variations of $\sigma$. An example of such an analysis of the PS density is shown in Figure 7.3 (a). Although in this example the structures - regions of high and low PS density - are more pronounced than typical, the general form, especially in the less pronounced areas is exemplary.

\section{Calculation of Expected Structuring}

In Figure 5 of the previous manuscript it was shown that PS reorganize along the interface between two dynamical regions. Just inside the region of lower excitability the NPS is strongly increased, while just outside it is reduced. Since this effect is expected to occur across regions of different excitabilities, and since the triangularity index of the action potentials appears to be a good measure for such heterogeneity, we may expect the PS re-organization to occur over regions where the triangularity index changes steeply. Thus, to convert the triangularity index $\Delta_{80}$ into a value for the expected structuring it seems advantageous to make a prediction of re-organization along areas where $\Delta_{80}$ changes.

First the $\Delta_{80}$ map itself is calculated as described in the methods Section 4.3.4. While the PS density is estimated from VF data, it is better to not estimate the $\Delta_{80}$ value from the same arrhythmia data, because the action potential shape is much less clear during arrhythmia and because possible confounding effects are avoided. Confounding effects might exist since the action potential shape may be different close to a spiral core. However, throughout the experiments local pacing events were recorded regularly when the heart was in sinus rhythm. This data - recorded without any present arrhythmia - can thus be used to characterize the dynamical heterogeneity. Here the same data and time spans as also analyzed in the previous chapters are used (compare Sec. 5.3 and 5.6). Thus the data contains:

1. Time spans ranging from 30 minutes to three hours and containing VF episodes of $3 \mathrm{~s}$ or $5 \mathrm{~s}$ duration

2. Individual $\mathrm{VF}$ episodes of $15 \mathrm{~s}$ to $30 \mathrm{~s}$ duration.

For the first data sets the $\Delta_{80}$ maps can be analyzed by using pacing events within the same time spans listed in the previous chapters. For the individual long VF episodes 
pacing events within 15 minutes of the episode are used to estimate the $\Delta_{80}$ map $^{1}$. All of the $\Delta_{80}$ maps calculated from these pacing videos are then averaged. Some further details are given below:

- The triangularity index is calculated from local pacing videos, in most cases with a $300 \mathrm{~ms}$ pacing period (in some cases, faster due to a fast sinus rhythm). The number of pacing stimuli varies, but is normally above 20 giving a good basis for analysis.

- It is possible that the heart does not follow the first pacing events or, rarely, that it does not follow reliably at all. For the analysis all action potentials are averaged to find the $\Delta_{80}$ map. Thus, it is possible that multiple action potentials of different shapes are averaged. However, here only the spatial differences in $\Delta_{80}$ matter. Thus, effects due to averaging action potentials of different shapes are not expected to affect the spatial heterogeneity. ${ }^{2}$

- The triangularity index can fluctuate strongly after a VF episodes due to the recovery process. Again, here, this effect is ignored since the spatial structure is largely independent of the absolute value.

After calculating the $\Delta_{80}$ map it is now necessary to compute an expected structure from it similar to the one in Figure 5 of the manuscript. To achieve this, one option is to use an inverted Marr wavelet or kernel function which is defined by:

$$
M(x, y)=-\frac{1}{\pi \sigma^{2}}\left(1-\frac{x^{2}+y^{2}}{2 \sigma^{2}}\right) e^{-\frac{x^{2}+y^{2}}{2 \sigma^{2}}}
$$

with $\sigma$ scaling the width (see Fig. 7.1(c)). The Marr wavelet is the scaled second derivative of a Gaussian function and is used for example in image analysis for blob detection. Applying it to a step in the excitability - as estimated from $\Delta_{80}$ - will show a similar behaviour along interfaces as seen for the PS density in the manuscript at the beginning of the chapter. An example of this analysis method is shown in Figure 7.1 and the expected structure calculated there is comparable to the findings shown in Figure 5 and Figure 4 of the manuscript. To avoid boundary effects in the video analysis, values outside the masked areas are filled with the nearest value from the same camera when applying the Marr wavelet.

Here I will use a kernel scaling size of five pixels $(\sigma=5)$ as indicated in the Figure 7.1(c). Unfortunately, there is no certain way to choose the best kernel size, and the size could vary between videos ${ }^{3}$. However, a wide variation of two to ten pixels still gives similar results.

\footnotetext{
${ }^{1}$ This removes a single time without such pacing data

${ }^{2}$ Note that failure of pacing affects the whole surface in a similar manner.

${ }^{3}$ Methods such as using the spatial correlation function may be used to evaluate and scale the typical size of the spatial patterns. Testing many different approaches, however, does not necessarily help when addressing the initial hypotheses, since it can also cause issues due to multiple testing.
} 
a)

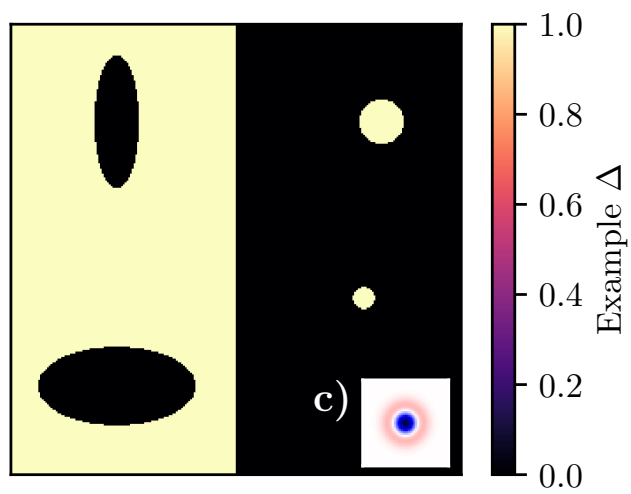

b)

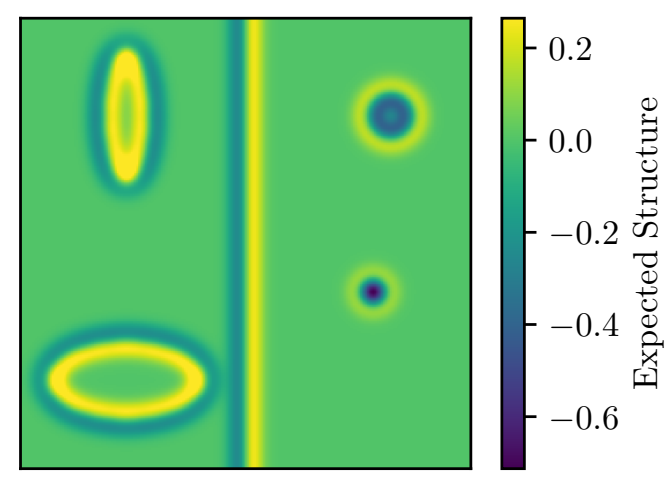

Fig. 7.1 a) A picture showing geometrical forms with a sudden exemplary change in the triangularity Index $(\Delta)$ between zero and one. The vertical line can be compared to the one seen in Figure 5 of the simulation analysis shown in the manuscript in Section 7.1. b) The result of the convolution with the inverted Marr wavelet. By highlighting the interfaces, the convolution result has a similar structure as seen in the simulation results. c) The small inset into (a) shows the kernel function with red values indicating positive weights, blue values negative weights and white zero weight. The spatial scales of all panels are chosen so that they can be directly compared to the experimental Figures 7.2 and 7.3.

Here, the analysis will mainly be based on this transform of the $\Delta_{80}$ map as this appeared to give the most stable results. However, similar results can also be achieved using $-\Delta_{80}$ directly. Both values are correlated and further study is required to know which one - or a combination of both - performs better. Fortunately, the exact choice of the model is not directly relevant to testing the initial hypotheses, since the aim is only to show that some type of relation appears to exist and not to precisely describe the relation.

\section{Quantification of the Spatial Structuring Strength}

To test Hypothesis 1, it is necessary to quantify the strength of both the heterogeneity in the PS density and the expected structuring due to the dynamical heterogeneity as calculated from the triangularity index. Here, this is done based on the standard deviation as a means to quantify the strength of the fluctuation. For the expected structure as calculated from $\Delta_{80}$, the standard deviation can be used directly as it is centred around zero. However, the PS density is naturally scaled by the NPS observed. Thus, instead of using the standard deviation, the standard deviation scaled by the mean value - the coefficient of variation - is used.

\section{Limitations}

The analysis of cardiac video data is fraught with difficulties. Spatial changes in signal quality or artifacts such as motion, could in principle produce similar artifacts in both 
a)

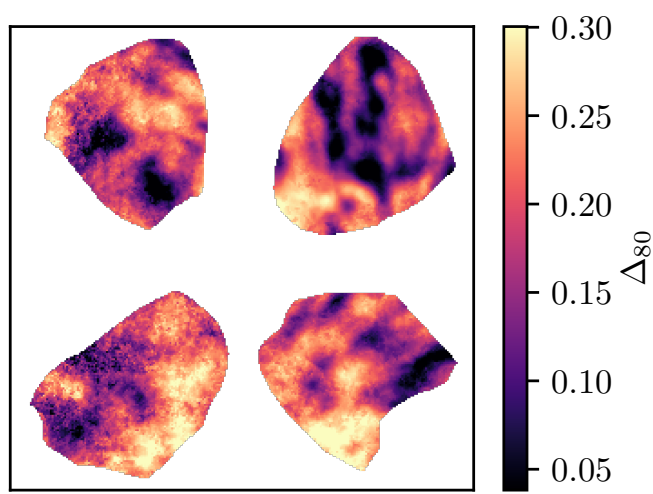

b)

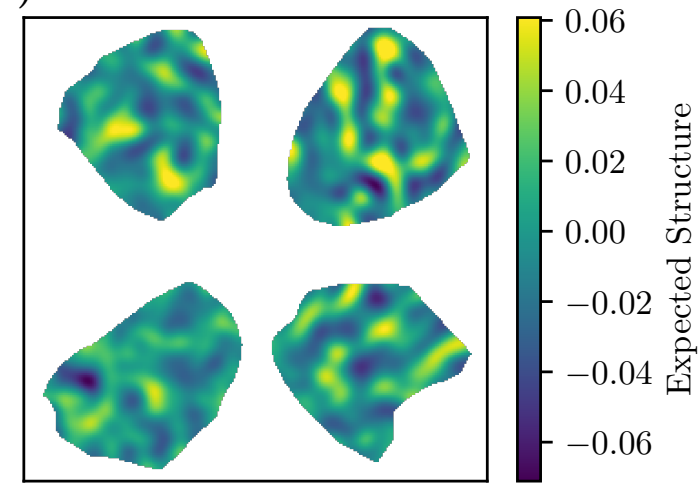

Fig. 7.2 a) Triangularity index $\Delta_{80}$ as calculated from pacing data recorded around the time of the VF episode 2017-09-28 at 12:08. Some areas of decreased triangularity index are visible for example in the upper right. b) Shows the result of the convolution with the inverted Marr wavelet which is designed to reveal changes of the spatial structure. The areas of low $\Delta_{80}$ have large values indicating that the PS density is expected to be higher there.

the PS density as well as the triangularity index calculation. While the PS density and the $\Delta_{80}$ analysis are based on different videos and thus on varying signal qualities, it cannot be fully ruled out that artifacts have a similar effect on both. Overall, it seems unlikely that there is an effect on the results since they are for example robust when limiting the analysis to a single camera view. This is a good sign, as the different cameras tend to have somewhat different image quality. Other limitations could arise due to the wrong choice of parameters. Fortunately, the results are robust against changes of the analysis parameters described in this chapter.

\subsubsection{Results}

Figure 7.2 shows the $\Delta_{80}$ map and the corresponding expected structuring as calculated using the convolution with the wavelet shown in Figure 7.1 and defined in Equation (7.1). This analysis smooths the $\Delta_{80}$ map slightly and gives clear maxima in those areas were the $\Delta_{80}$ value is low but surrounded by a higher value.

Figure 7.3 shows an example of the PS density and the same expected structurization. In this $30 \mathrm{~s}$ VF episode recorded on the 2017-09-28 at 12:08 there is a very dominant area where many - often relatively short lived - PS are detected. This area is located on the RV (lower left of the image) and is associated with slow wave propagation leading to many PS being found. While this region also has an increased value in Panel (b) it is not as prominent there. However, the contour lines in Panel (b) show that in many other areas the structures are similar in both (a) and (b). In this VF episode the correlation between the expected structure and PS density is 0.33. 
a)

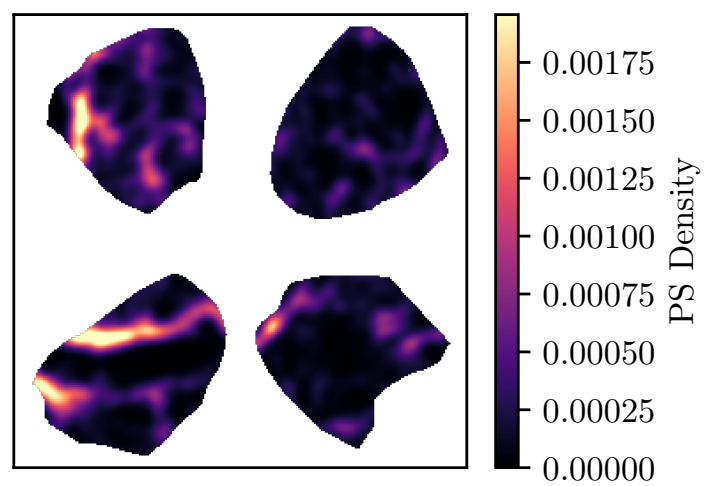

b)

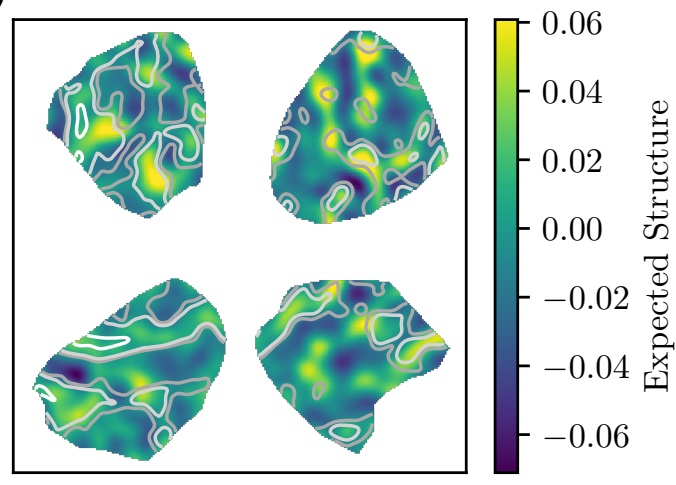

Fig. 7.3 a) The spatially smoothed PS density for the $30 \mathrm{~s}$ VF episode on the 2017-09-28 at 12:08. The density is high in the lower left of the plot - on the RV. In this area slow wave conduction commonly causes the detection of short lived PS. b) The result of the convolution with the inverted Marr wavelet (same as Fig. 7.2(b)). The additional contour lines indicate levels of the PS density for easier visual comparison. The spatial correlation coefficient between (a) and (b) is 0.33 .

\section{Statistical Analysis of all Episodes}

The analysis can now be repeated for all episodes ${ }^{4}$ and time spans analyzed in the Pinacidil series. Figure 7.4 discusses the two hypotheses stated at the beginning of the chapter:

1. Panel (a) compares how strongly the observed PS density is structured with how much structuring is expected from the $\Delta_{80}$ heterogeneity by applying the convolution with the Marr wavelet. There appears to be a strong linear trend with increasing values. This linear correlation can also be tested using a one sided $t$-Test based on Pearson's correlation coefficient ${ }^{5}$. The result of this analysis gives a $p$-Value below 0.005 . This is also true when limiting the analysis by removing the single videos (stars) - although not when limiting it only to the stars. While these $p$-Values should be taken with care due to the complexity of the overall video analysis, the behaviour of increased structure in the PS with increased dynamical heterogeneity seems compelling. ${ }^{6}$

2. Panel (b) shows the correlation coefficient between the expected, i.e. derived from the $\Delta_{80}$, and real PS density. While the correlation coefficient is generally small, it is in almost all cases positive so that a random fluctuation - around zero - can be ruled out. In other words, the null hypothesis of the analysis

\footnotetext{
${ }^{4}$ With the exception of the VF episode on the 2017-09-21 at 11:50 for which no pacing data was recorded within the time span of 30 minutes.

${ }^{5}$ Here the R command cor.test was used [107]. Similar results are found using rank based tests such as Kendall's $\tau$.

${ }^{6}$ For example they are based on multiple data points from the same heart. However, the spatial structure often varies strongly at least when comparing early to late VF episodes.
} 
a)

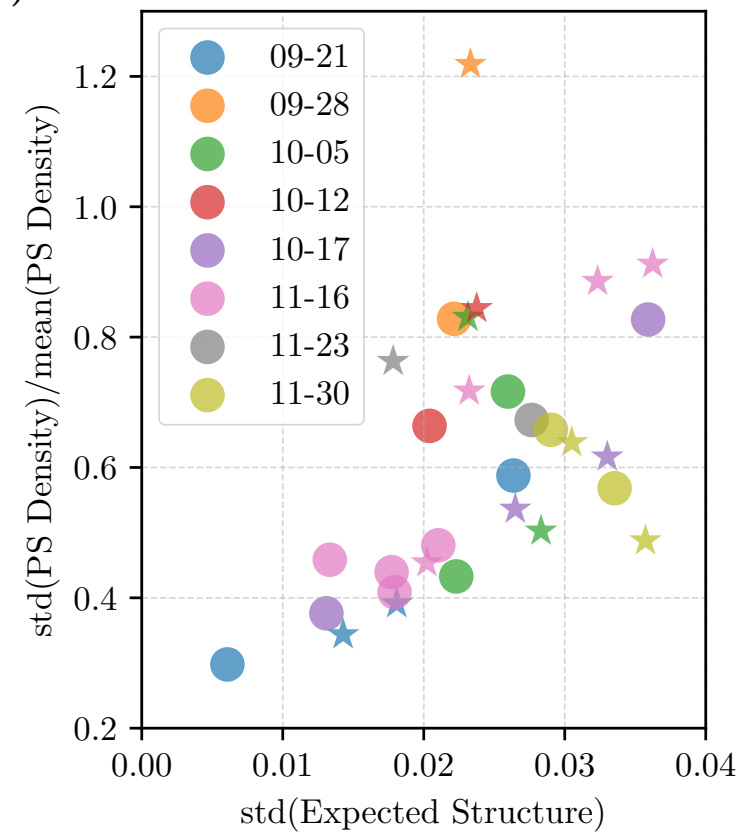

b)

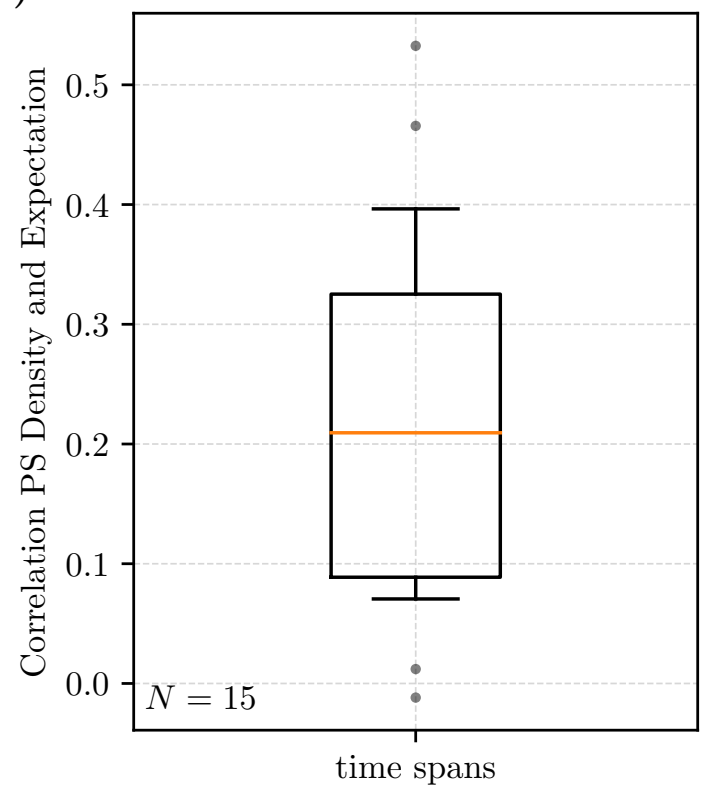

Fig. 7.4 a) The strength of the structuring seen in the PS density plotted against the expected structuring strength estimated from the $\Delta_{80}$ heterogeneity. A clear increasing trend is seen. Circles indicate time spans with many VF episodes while stars indicate a single long VF episode. The colours separate the experimental days, and the legend also applies to the stars. b) The spatial correlation coefficient between the PS density and the expected structure as derived from the $\Delta_{80}$ map. The two boxplots show the result of the time spans containing multiple VF episodes and the single long VF episodes. In total only very few out of 30 values show a correlation coefficient below zero.

finding both positive and negative correlation values with equal probability can be rejected.

The identical plot based on the analysis of the APD and without applying the Marr wavelet transformation can be found in Appendix A.5 (p. 153).

\subsubsection{Discussion of the Spatial Heterogeneity Results}

Considering the results shown in Figure 7.4 and the related statistical test, the results presented in this chapter clearly suggests that there is a relationship between dynamical heterogeneity - local changes of the tissue properties - and the spatial PS density. The generally positive spatial correlation between the expected and real structure of the PS distribution could on its own be explained by a common cause. Such a common cause could for example be the position of blood vessels. However, blood vessels are always present so that they cannot as readily explain a common increase in the fluctuations of both dynamical heterogeneity and PS density. The combination 
of both hypotheses thus makes the argument much stronger by pointing to a direct causal relationship between the two. The nature of this causality is, however, not studied here. Another result is that the analysis was more successful by applying the transformation based on the Marr wavelet as inspired from the theoretical manuscript. However, unlike in the manuscript it is not clear to which degree the structure is an effect associated with the interface rather than due to a higher number of PS in the ischemic regions. Likely both effects play a role.

Previous studies observed PS occurrence in preparations including regional ischemia [108-110] or relate an increased occurrence of VT to ischemia or myocardial infarction [111]. Here, the same is shown for spontaneous arising heterogeneity which is most likely related to ischemia as well. However, to the best of my knowledge no previous study has directly studied the first hypothesis or performed a statistic test to prove that such heterogeneities are in fact important to the spatial structure of the arrhythmia. Unlike works studying heterogeneities as causes of arrhythmia or properties related to it, here the effect on the developed VF is analyzed.

Thus, the results show with a high certainty that dynamical heterogeneity does affect the spatial organization of ventricular fibrillation in the rabbit heart. It shows that simulation studies as our manuscript above [106] and studies cited therein such as [112, 113] may indeed be important for a detailed understanding of cardiac fibrillation.

\section{Outlook}

While evidence for the spatial structuring in the PS due to dynamical heterogeneities is interesting, further analysis could help to better understand its nature and quantify the strength. The heart is highly anisotropic so that the circular Marr wavelet used here to find the expected structure is unlikely to be the best approach. Rather, a scaling based on the spatial correlation function may achieve a higher correlation coefficient between PS density variations and functional heterogeneity. The model for the expected structure was inspired by the structuring along a step boundary in a numerical model. However, the changes of the dynamical properties are in reality smooth and not of a binary nature. Thus, it could be interesting to test whether the model used here can be modified to better capture the reorganization occurring along smooth boundaries. 


\section{Chapter 8}

\section{Controlling Cardiac Arrhythmia}

Control of the cardiac dynamics during the arrhythmia can be exerted using local stimuli or far field shocks. When the termination of the arrhythmia is of interest, far field shocks are the most important method. In this chapter the perturbations and effects that far field shocks have on the developed ventricular fibrillation will be discussed.

Electric shocks used for defibrillation are applied either with paddle electrodes from the outside, or by an implanted defibrillator. In the case of the implanted defibrillator different shock vectors exist although one electrode is always on a catheter placed into the right ventricle of the patient.

The general effect of the shock, zones of hyper- and depolarization being created by the current passing between the paddles, is well known both in experiments and simulations, as described in the introductory Section 1.5. Activation maps, as shown briefly also in the previous Section 5.5, provide an understanding of where a shock can recruit wave emitting sites [4]. Further, simulation studies and theories give insights into more detailed mechanisms and recruitment patterns [18-20].

Effects, such as the excitable gap and the vulnerable window, which describes when a shock can affect the local dynamics or a spiral wave (compare Sec. 1.5), are well studied $[24,60]$ and were shown to affect the response to local pacing in experiments [22]. However, the actual recruitment patterns of far field shocks, which are at the core of defibrillation methods, are more elusive. Since fibrillation is a chaotic state, the effect of one shock will never be the same as the next. For example, a successful termination has a vastly different outcome than an unsuccessful one. Although case studies can highlight paths of defibrillation failure compared to its success, general assertions are difficult to make.

It is thus desirable to learn more about the recruitment of new waves caused by the depolarization zones from the electrical shock. One approach to find these recruitment patterns in optical mapping is to use statistics over many shocks. To this effect, I have performed and analyzed experiments by:

- Focusing on unsuccessful termination events since they allow rapid repetition. 
- Assuming that the perturbation of a non-terminating shock decays within a few typical cycle lengths of the arrhythmia.

- Finding the activation patterns after shocks and using statistics over the repetitions.

Shock protocols for this analysis were performed in several experiments. The analysis performed here always focuses on using the excitation times as the basis of analysis as shown in Section. 4.2. Based on this, the probability of an activation having occurred within a given time window is calculated. This time window normally consists of the ten milliseconds after a shock was applied. Many of the following results can certainly also be made by analyzing the fluorescence data more directly. However, the approach of calculating a probability provides clear interpretation and is thus used throughout this chapter for consistency.

\section{Limitations}

As noted before, most analysis here is based on the extraction of excitation times and smoothing as previously described in Chapter 4. One additional limitation arises here due to the shock itself. Due to a higher spatial coherence of activation and the depolarization caused by the shock, activation originating from a shock can have a larger amplitude than typical fibrillatory activity. To some extent focusing on the excitation times should make the analysis more robust in this regard. In some events, an activation that started before the shock, but is then enhanced by it, maybe falsely interpreted as having occurred after the shock. Also it sometimes seems that large amplitude shocks can cause an upstroke in tissues that would normally be assumed refractory. ${ }^{1}$ Since the excitation detection algorithm uses the assumption that excitations must be separated by a refractory time and chooses the most prominent one, this leads to missing the previous activation.

An experimental limitation is the trade-off between the waiting time between shock events, risking that the arrhythmia is still perturbed by the previous shock, and the maximizing of the total number of shocks for good statistics. While effects from previous shocks were not observed, they might nevertheless have affected some of the faster paced results.

A more general limitation is the use of optical mapping, limiting all discussions to epicardial observations.

\section{General Setup}

Figure 8.1 again sketches the general setup used in the experiments. It shows a section through the heart when viewed from below, so that the camera placement is the same

\footnotetext{
${ }^{1}$ Especially since the imaging reaches relatively deep into the tissue, it seems possible that some cells or sheets of cells skip activations as the effective imaging area averages over many cells. I believe similar observations were shown by Godfrey Smith during the 1st Be-Optical School in 2016 in Göttingen: Using two-photon excitation his team observed that some cells skipped beats while others close by did not.
} 


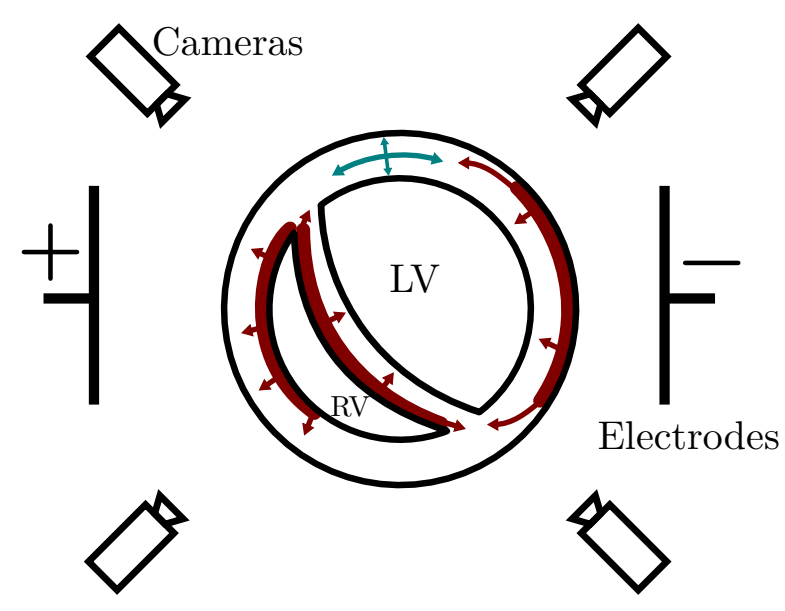

Fig. 8.1 Sketch of the setup showing a cross-section of the heart, cameras, and electrodes looking from below (the apex). RV indicates the right and LV the left ventricular chamber. The plate electrodes that deliver shocks are indicated on the left and right sides. Areas show in which activation is expected following an electric shock are shown in red. In the experiments, the orientation of the heart was always prepared to be similar to the sketch. Blue arrows indicate general wave propagation, it may be that wave propagation occurs more commonly along the wall than from the inside to the outside (transmural).

as in the result images. Two plate electrodes with a distance of $9.5 \mathrm{~cm}$ were used to deliver monophasic, rectangular shocks of $5 \mathrm{~ms}$ duration (compare Sec. 2.4). The dark red colour approximately indicates areas where important activation sites are expected [18-20] as well as the wave propagation from these. The blue arrows indicate wave propagation directions which is used for discussion later in this Chapter.

\subsection{Visualizing Shock Induced Excitation}

A beautiful example of the excitation structures was seen using this approach on data from an experiment performed by Daniel Hornung using my shock protocol ${ }^{2}$. During this experiment 100 shocks with a pause of half a second between them were made during a single VF episode. This was done first at a voltage of $10 \mathrm{~V}$ and then at $20 \mathrm{~V}$. After finding the times since the last excitation occurred, as described in Section 4.2, it is possible to extract the probability of a point being activated within $10 \mathrm{~ms}$ after the shock. This is calculated by first finding all points activated during the $10 \mathrm{~ms}$ after each shock. Then, for each pixel, the probability of activation is calculated over all shocks.

The results of applying this procedure on the data recorded for both voltages are shown in Figure 8.2. In most areas of the heart, the probability of excitation is unstructured and has values of about 0.1. This is expected since during unperturbed VF every point is activated approximately every $100 \mathrm{~ms}$ giving a $10 \%$ probability of

\footnotetext{
${ }^{2}$ Experiment performed 2017-07-10.
} 
10 V Shock

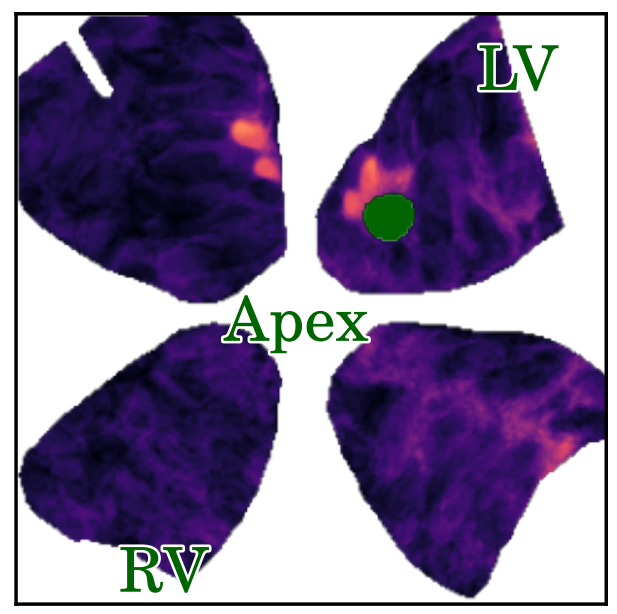

20 V Shock
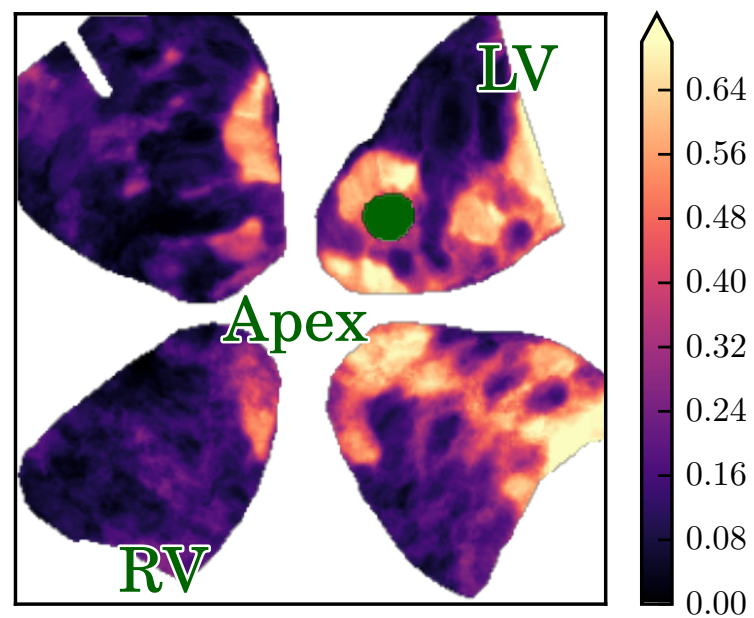

Fig. 8.2 Probability of an area being activated in the first $10 \mathrm{~ms}$ after a shock of the corresponding Voltage is given. The green area indicates a cryo-ablation zone (no activation possible). The probability is calculated by averaging over 100 shocks for each of the images. Both measurements were performed within a single VF episode.

activation within the time span of $10 \mathrm{~ms}$. For the $10 \mathrm{~V}$ shocks the main observation is a small wave source appearing at the cryo-ablation zone ${ }^{3}$ marked in green. This area is activated for about $40 \%$ of the shocks. Increasing the voltage to $20 \mathrm{~V}$ increases the effect around the cryo-ablation zone considerably. Additionally, large areas oriented towards the anode also have a high probability for being recruited (compare Fig. 8.1).

These recruited wave emitting sites can also be compared to those when the heart is quiescent. During this experiment activation maps using shocks of comparable strength and the same pulse width ${ }^{4}$ were recorded. The activation map in quiescent tissue also shows the first activation close to the surface ${ }^{5}$ at the cryo-ablation zone. In quiescent tissue activation at the cryo-ablation zone occurs at a voltage between $5 \mathrm{~V}$ and $6.3 \mathrm{~V}$. The areas which are recruited during $\mathrm{VF}$ at $20 \mathrm{~V}$ as seen in Figure 8.2 are activated starting around $15 \mathrm{~V}$ in quiescent tissue.

Thus, it is possible to directly image those areas that can act as a wave emitting site during the arrhythmia itself. Further, this analysis additionally provides the probability of activation. This detail will be analyzed more closely in Section 8.4.

It is not surprising that there is a strong correspondence of the results during VF when compared to those for quiescent tissue. However, the analysis above confirms that the activation sites indeed remain similar during VF.

\footnotetext{
${ }^{3}$ In this area tissue was ablated using metal cooled with liquid nitrogen.

${ }^{4}$ The shock period of the activation map was $0.5 \mathrm{~s}$, the pulse width and polarity were identical to the shocks. The voltage steps were slightly different.

${ }^{5}$ The first actual activation is likely at the apex but not close to the surface as indicated by a large time until activation. Similar to the activation map shown in Figure 5.3
} 

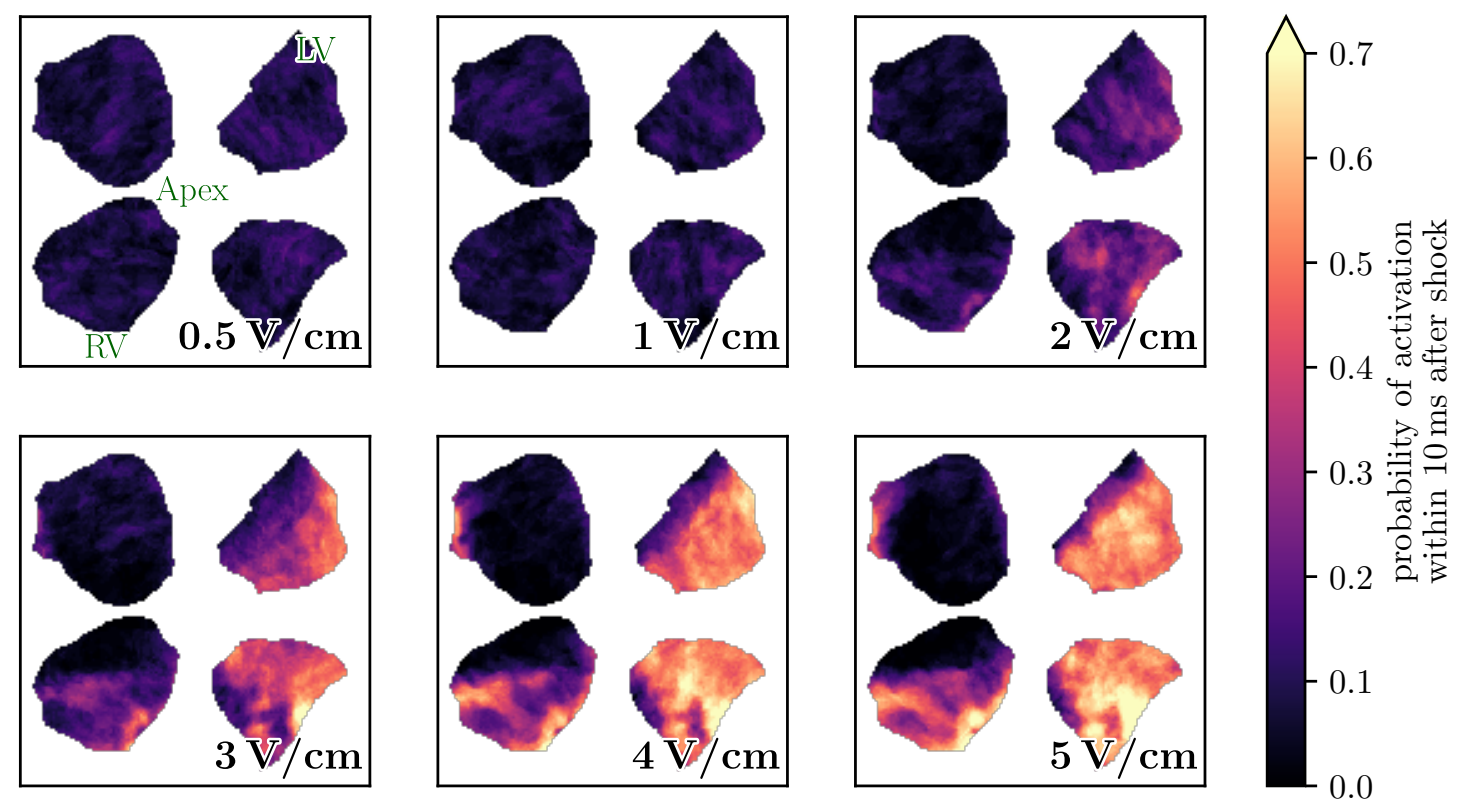

0.0

Fig. 8.3 Probability of an area being activated in the first $10 \mathrm{~ms}$ after a monophasic $5 \mathrm{~ms}$ long shock of varying Voltage is given. The probabilities are calculated from 70 events for each panel, and these repetitions were split over multiple VF episodes (each VF episodes would contain all voltage values). Shock strengths are indicated in the lower right in $\mathrm{V} / \mathrm{cm}$ with $1 \mathrm{~V} / \mathrm{cm}$ corresponding to $9.5 \mathrm{~V}$.

\subsection{Recruitment and Wave Propagation after the Shock}

In this section, results from a different experiment will be presented. In the experiment more and higher voltages were tested. First, the results of the previous section will be confirmed. Then I will take a closer look at the wave propagation just after the shock occurred.

In this experiment ${ }^{6}$ eight separate episodes of $\mathrm{VF}$ were measured with shocks ranging from $0.5 \mathrm{~V} / \mathrm{cm}$ to $5 \mathrm{~V} / \mathrm{cm}$ with $1 \mathrm{~V} / \mathrm{cm}$ corresponding to $9.5 \mathrm{~V}$. Within each episode all voltages were tested at most 10 times in a randomized order. The VF was given a time of $2 \mathrm{~s}$ to recover after each shock. In total 70 shocks are analyzed for each voltage. Very occasionally defibrillation occurred and was reinduced again quickly. Since the statistics are over a large number of events, these, however, have no significant effect on the outcome.

Figure 8.3 shows the result of the analysis as done in the previous section. At low voltages up to $1 \mathrm{~V} / \mathrm{cm}(9.5 \mathrm{~V})$ little or no activation can be seen within the first $10 \mathrm{~ms}$ after the shock was applied. At larger voltages, the affected areas as well as the probability of activation increase dramatically. This again confirms the expectation

\footnotetext{
${ }^{6}$ Experiment performed 2018-08-22.
} 
from activation maps or simulations about which areas are recruited for new waves, although in this heart the activation pattern results have less clear structures.

Instead of a snapshot as seen Figure 8.3 it is now also possible to shift the $10 \mathrm{~ms}$ window to later times after the shock. In this case wave propagation originating from the first activated sites becomes visible as an increased activation probability in neighbouring regions with a small delay. As the activity evolves after the shock, the probability of activation will also decrease again in the initially activated areas. Figure 8.4 captures this analysis. The first panel shows the maximum probability of activation found within any $10 \mathrm{~ms}$ window during a time of $100 \mathrm{~ms}$ after the shock. Thus, an area which is commonly activated at a later time after the shock will also have a large probability in the figure. Looking at this analysis, one finds that also in the left side of the plots (right side of heart) areas are eventually activated with a relatively high probability. Part of this area is indicated by a green square in the $5 \mathrm{~V} / \mathrm{cm}$ panel.

To capture the temporal information, Figure 8.4(b) shows the time span when the maximum activation probability occurred, with $0 \mathrm{~ms}$ indicating the probability of activation was maximal within $0 \mathrm{~ms}$ to $10 \mathrm{~ms}$ and $10 \mathrm{~ms}$ indicating a time span from $10 \mathrm{~ms}$ to $20 \mathrm{~ms}$ and so on. Especially at low voltages the times vary widely since the shock is dominated by the random fluctuations of the VF. However, the later panels with higher voltages show clear structures. First, they clearly show the initially activated areas. Then, waves spreading from these sites create areas of increasing times in the neighbouring regions. These regions also have large values in (a). Further, the area indicated by the green square in the $5 \mathrm{~V} / \mathrm{cm}$ panel of (a) has the highest probability of activation at times around $20 \mathrm{~ms}$ to $40 \mathrm{~ms}$. This may be linked to wave sources that are recruited within the right ventricle on the endocardial wall, which require a short time to propagate to the epicard. Another interesting observation is in areas orthogonal to the shock electrodes (top and bottom of the plot). These areas are indicated by green arrows in the $4 \mathrm{~V} / \mathrm{cm}$ panel of (a), and in them the probability of activation never reaches high values even at large voltages.

This phenomenon warrants some more discussion. On the one hand, it is known that less recruitment is expected to occur in these areas. However, one may expect a larger probability of waves propagating into such an area from those sites that are activated close by. One contribution to this low probability of activation may be that different activation paths have different length and propagation times. Further, it may be that no activation sites lie close to these areas inside the heart. Contrary to the orthogonal areas with a low excitation probability, for the main activated sites aligned towards the electrodes activation is expected to occur in large areas on or close to the wall itself. Thus, the relative distance to the closest wave emitting site may be larger. Also, Niels Otani and colleagues [114] suggested wave emission from the wall in an orthogonal direction to the direction of wave propagation as a mechanism for removing spirals from the heart and thus to defibrillate. A similar pattern as suggested by them may be an additional reason for the differences in the shock effect. The results shown highlight the importance of the shock geometry. The localized nature by which shocks 
a)
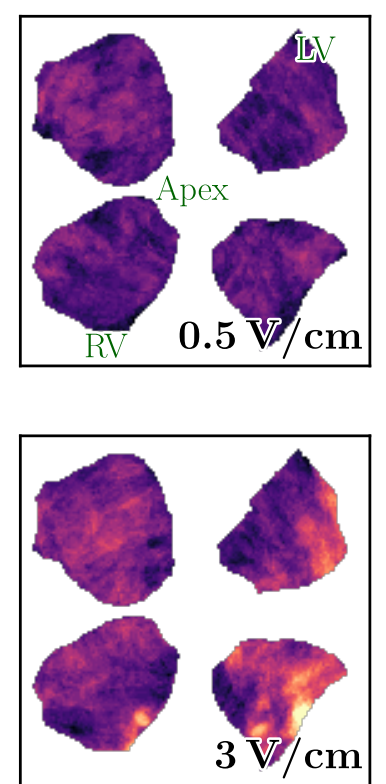

b)
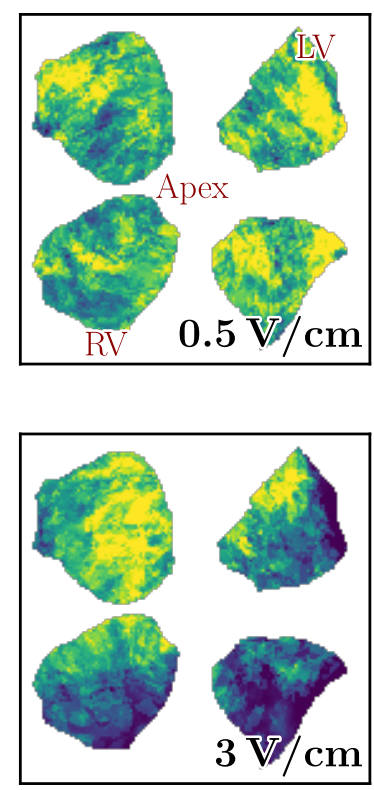
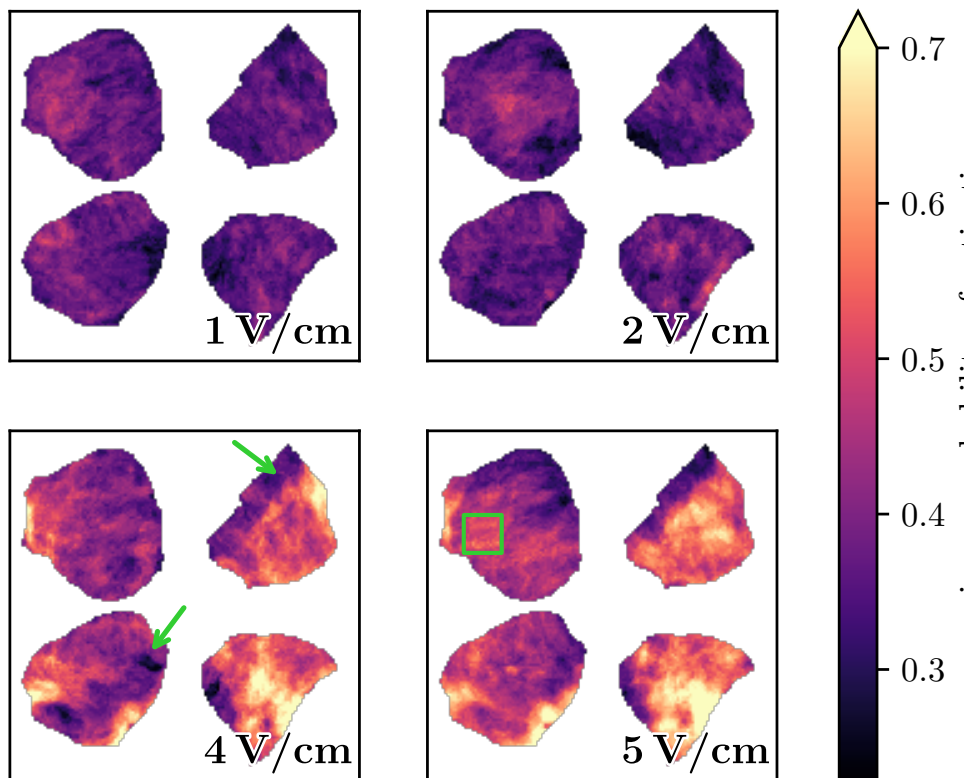

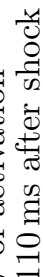

0.5 总
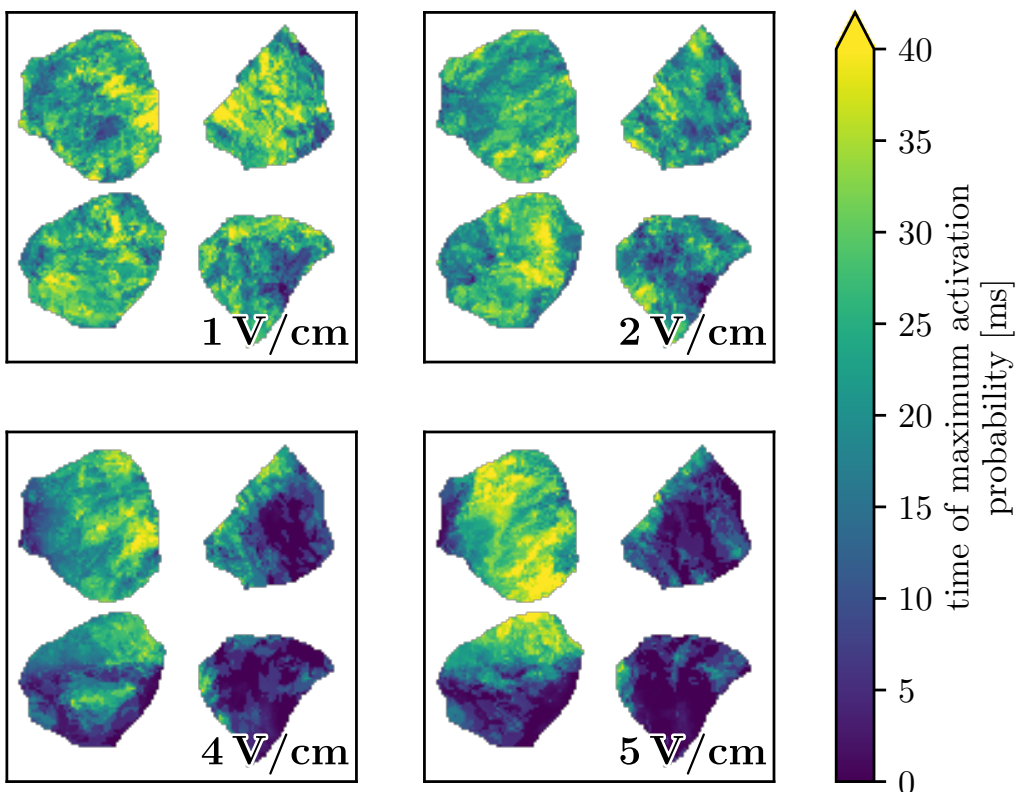

Fig. 8.4 a) Probability of an area being activated during any $10 \mathrm{~ms}$ time span within $100 \mathrm{~ms}$ after a shock of different voltage is given (moving windows). It is thus an extension of Figure 8.3 showing always larger values, since sometimes areas are commonly activated at a later time. b) The time when the maximum activation probability shown in (a) occurred. Thus, for example a value of $10 \mathrm{~ms}$ indicates that it was most likely to find an activation to occur in the time range of $10-20 \mathrm{~ms}$ after the shock. This indicates wave propagation and later activation on the left side of the plot (right side of the heart). 
affect the tissue are an important information that supports theoretical considerations for designing new shock vectors such as those suggested by Otani.

\subsection{Comparison of Shock Protocols}

Visualizations and comparison of different shock protocols may be another useful application. One open question is whether it is advantageous to give multiple shocks faster than the typical frequency of the arrhythmia (overdrive) or slower (underdrive). This has been studied extensively in experiments and simulations, for example in $[33,115,116]$.

The results of such an analysis can be seen in Figure 8.5 showing the area activated within $10 \mathrm{~ms}$ for each of 5 shocks. ${ }^{7}$ Here a total of 25 shock sequences were performed within a single VF episode, first as over- then as underdrive shocks, meaning either faster or slower than the typical frequency of the arrhythmia. Each sequence of overand underdrive consisted of 5 shocks at an amplitude of $20 \mathrm{~V}$. Overdrive shocks were given at a period of $96 \mathrm{~ms}$ and underdrive shocks at a period of $131 \mathrm{~ms}$. Between each of the shock sequences the VF was allowed to recover for a time of at least $0.5 \mathrm{~s}$. Note that the polarity is reversed compared to other recordings, so that activation is more pronounced on the right ventricle or left side of the image, when compared for example to Figure 8.2.

The effect of the first shock should be equivalent for over- and underdrive pacing, since the state before it is assumed to be chaotic and unperturbed arrhythmia. This is also what can be seen in the corresponding panels of Figure 8.5 which are very similar. However, the activation patterns differ greatly for subsequent shocks. In the case of overdrive pacing, the same areas are directly recruited by each shock with an increasing probability, while the effect on other regions decreases. This can be explained by waves originating from the prominent areas. After the first shocks the same main sites are recruited for every shock. These waves then travel over much of the heart creating zones that are activated at a later time, and thus are still refractory when the next shock comes. Although the main activated sites appear not to change anymore after the first few shocks, closer inspection shows that the activation patterns outside these areas are not activated as systematically (compare Sec. 8.2) as the main activation sites. It appears that the synchronization of the main recruited areas can be rapid, while synchronizing of tissues not part of these main sites is slow or may not occur at all.

In contrast to the overdrive example, the picture is more diverse in the underdrive case. While similar regions are still excited, in most parts the shocks are too slow to activate these regions every time. One small area towards the right is an exception and is activated almost every time. This exception is explained by a locally larger cycle length of about $125 \mathrm{~ms}$ compared to otherwise 85 to $110 \mathrm{~ms}$, and, although less clear, by a longer $\mathrm{APD}$ with an approximated $\mathrm{APD}_{50}$ of above $50 \mathrm{~ms}$ when much of the heart

\footnotetext{
${ }^{7}$ Experiment performed on 2017-08-11 mainly by Daniel Hornung with my additional shock protocol.
} 


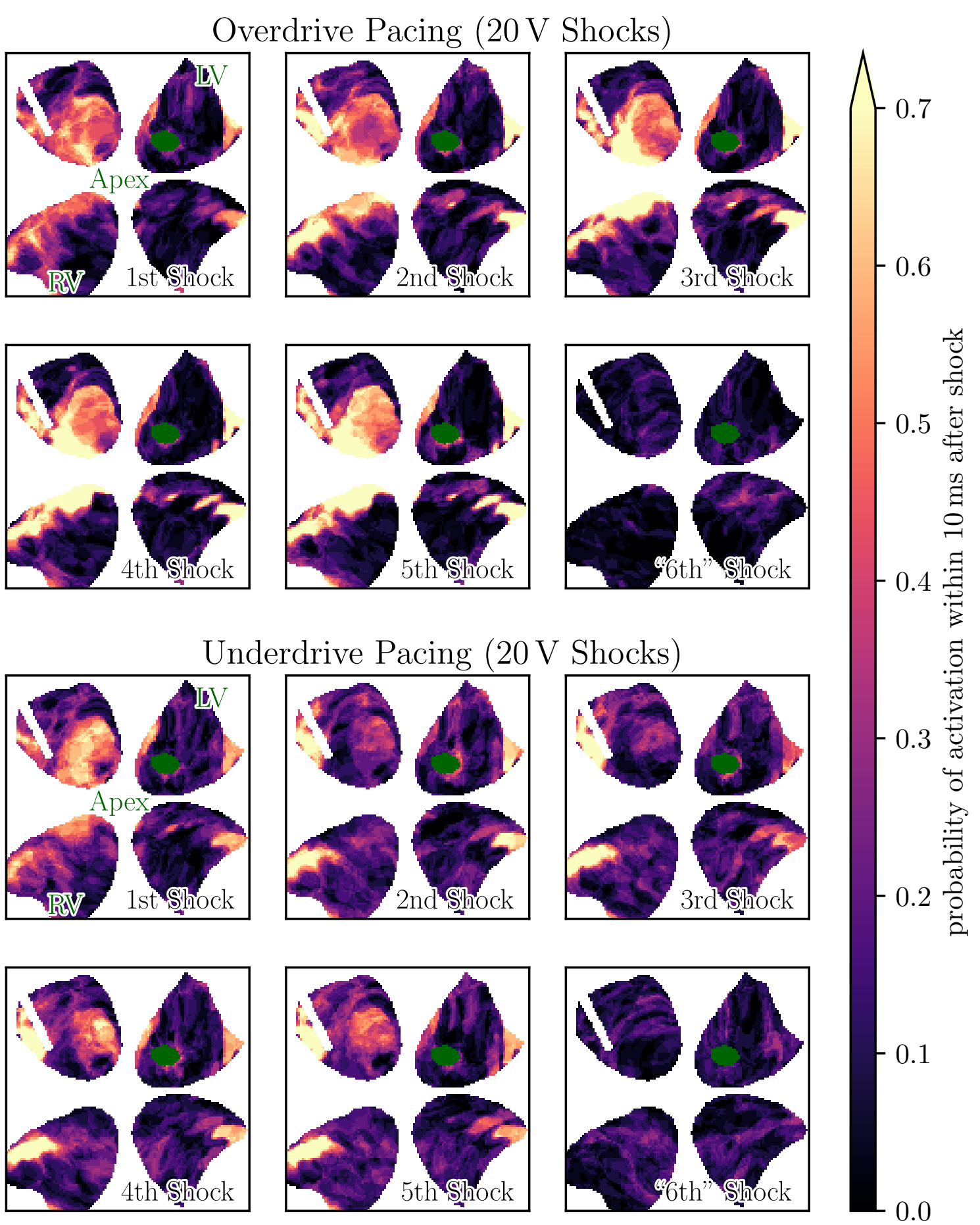

Fig. 8.5 Probability of activation occuring within $10 \mathrm{~ms}$ after each shock in a series of 5 shocks. Statistics calculated from 25 events each. In the upper rows shocks were given at a period of $96 \mathrm{~ms}$ between shocks and thus faster than the dominant CL of the VF (about $110 \mathrm{~ms}$ ). In the lower rows shocks were given at a period of $131 \mathrm{~ms}$ between shocks and thus slower than the CL of the VF. The polarity of the shock is inverted compared to the most other examples leading to a more dominant activation towards the left side of the image (right side of heart). The excitation at the period at which a "6th" shock would have been given is also shown. The green area identifies a cryo-ablated region (no activation possible). 
has a very short one below $30 \mathrm{~ms}$. Since there is a lower dominance of sites which are recruited every time, some areas such as toward the bottom of the images (back of the heart) which are roughly orthogonal to the shock vector are activated occasionally. Overall underdrive shocks appear to lead to a less strong but more diverse recruitment pattern from one shock to the next.

For comparison, the activation pattern one period after the 5th shock ("6th") is also given. Some patterns can be seen, indicating remaining effects of the previous shocks. However, the overall probability of activation is low, showing that the coherent activation of specific sites is largely lost even within a single period.

For the most part these results confirm the expectation about how shocks may exert control on the cardiac tissue. However, the differences between the protocols seen here indicate that new information can be gained with such analyses.

\subsection{Quantification of the Excitable Gap for Far Field Shocks}

In the previous sections the probability of excitation shortly after the shock was shown and a high probability was interpreted as a zone where excitation occurs directly due to the shock. This probability is affected by the number and places where a shock can create new waves, as well as how susceptible the tissue is at the time of the shock. The former can also be obtained from activation maps and times where shocks are given when the tissue is quiescent. Activation time and map analysis for the Pinacidil experiments also used here are shown in Section 5.5. There, no effect of Pinacidil on the activation times could be found, thus indicating that the number of recruited wave emitting sites is largely unaffected by Pinacidil. The latter influence of the susceptibility to the shock is due to the excitable gap. The excitable gap describes the time after an excitation when a cell can be excited again and before the next natural excitation cycle occurs. Since a stronger forcing has a larger effect on the cell, this time window increases with the applied shock voltage. However, it is also an important characteristic of the dynamics. Shortened action potentials, as seen with Pinacidil, may cause an increase of the excitable gap. In literature the size of the excitable gap is sometimes measured as the effective refractory period, the minimum time after which a local stimulus can excite the tissue again [27, 117]. To study how Pinacidil, which was used the in previous result chapters, affects the excitable gap, here, it is analyzed via its effect on far field shocks which are also used during defibrillation.

Using the statistical approach from before, the hypothesis of an increased excitable gap due to Pinacidil can be examined. Figure 8.6 shows the probability of activation shortly after a shock of increasing strength with and without Pinacidil for the 2017-10-05 experiment. ${ }^{8}$ The direct comparison shows similar patterns for both the

\footnotetext{
${ }^{8}$ Due to small inaccuracies - mostly well below a single camera frame of $2 \mathrm{~ms}$ - for this specific analysis activation was considered to be after the shock even when it was detected within one camera
} 

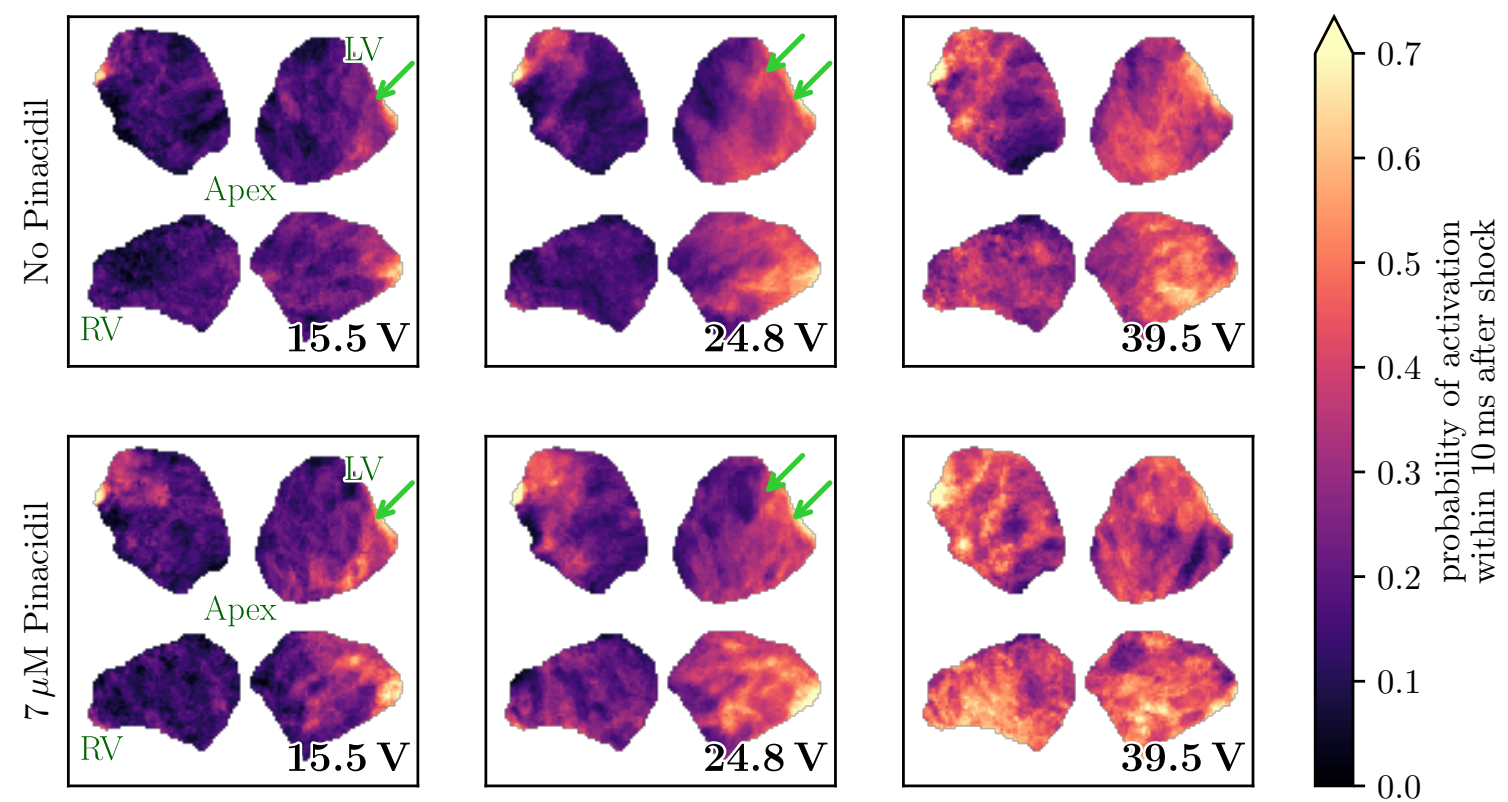

Fig. 8.6 Probability of excitation within $10 \mathrm{~ms}$ after a shock of various amplitudes during the 2017-10-05 Pinacidil series experiment. The top row shows the probability for an episode without Pinacidil (at 17:06) while the bottom row was measured with $7 \mu \mathrm{M}$ Pinacidil (at 16:07). The first and last panel provide statistics over 50, the centre one over 100 shocks. All shocks were given within the same VF episodes (one with and one without Pinacidil). After each shock the VF was allowed to develope freely for a minimum of about $650 \mathrm{~ms}$. Green arrows indicate areas of high activation probability added at the increased voltage (compare text).

Pinacidil and no Pinacidil conditions with generally higher values in the Pinacidil case. As mentioned, the sites that are recruited are not expected to differ between the two cases for the same voltage. This similarity in recruitment is visible on close inspection of the patterns in the top right (left ventricle) when going from $15 \mathrm{~V}$ to $25 \mathrm{~V}$. In both cases increasing the voltage adds a second "stripe" of higher probability. These stripes are indicated by green arrows.

To further elucidate and quantify the effect, Figure 8.7 shows the spatial histogram over the areas first activated by the shock. This area is approximated as the right side in the images shown in Figure 8.6. The figure includes the same analysis for two more experiments in the series. The histogram shows the area fraction which is activated with a given probability. For higher voltages more of the area is activated with a high probability leading to a shift of the histograms towards large probabilities (right). A similar effect is also seen for Pinacidil. Comparing the histograms calculated from the shocks with and without Pinacidil show that much larger regions are activated with a

frame before the shock should have occurred (compare limitations section). The time span chosen still represents $10 \mathrm{~ms}$, but is thus slightly shifted. This was necessary due to a relatively low video quality during the Pinacidil measurement. 
a)
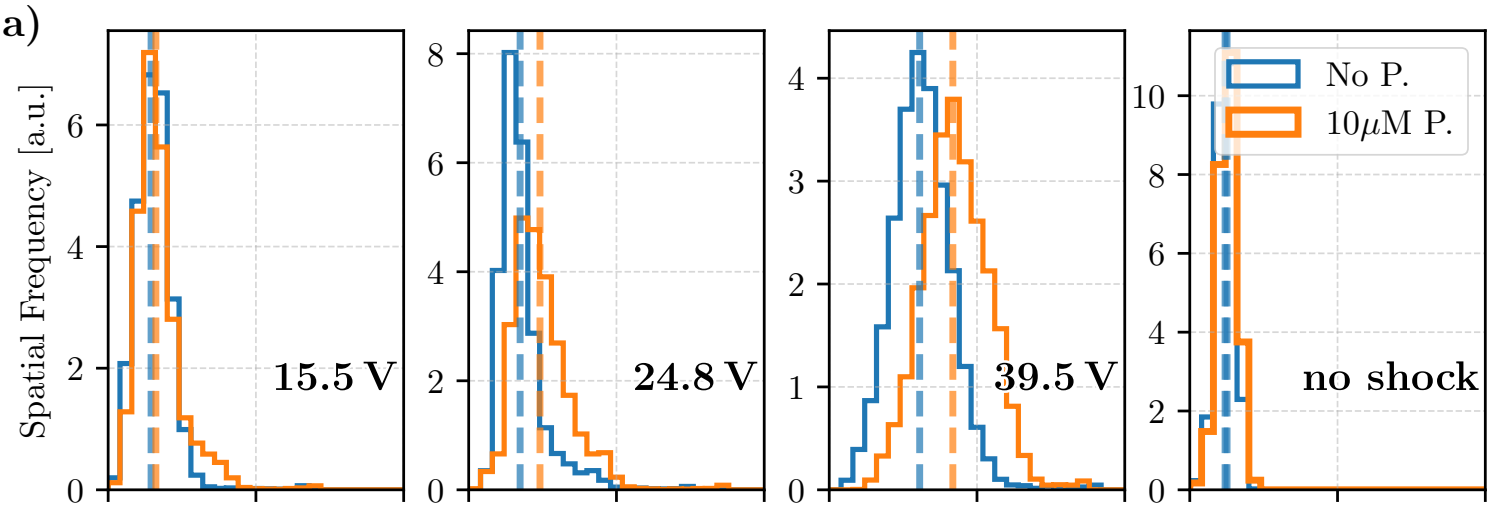

b)
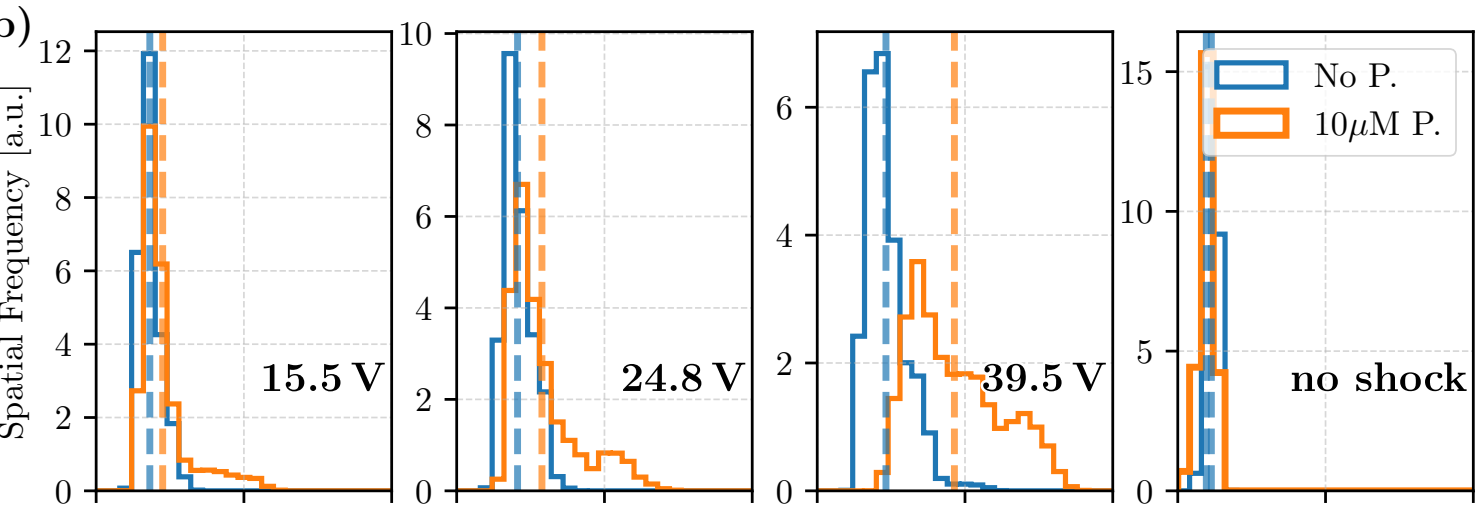

c)
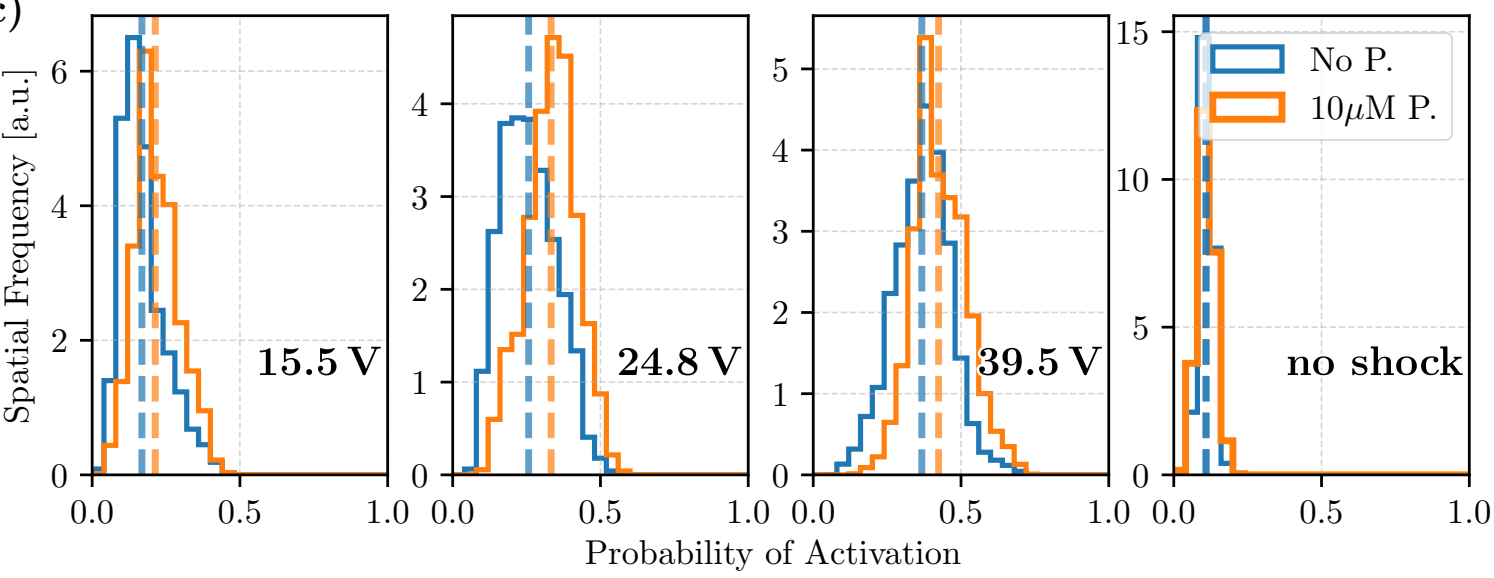

Fig. 8.7 a) Spatial histogram over the values shown on the right cameras in Figure 8.6 from the 2017-10-05 Pinacidil experiment. The right cameras roughly correspond to the area first activated by the shock. Thus it shows the area fraction that is excited with a given probability within $10 \mathrm{~ms}$ after a shock. The shock voltage increases from left to right. Additionally, the distribution shortly before the shock is given for comparison. b) Corresponding histogram for the experiment 2017-09-21 averaging 150 (15.5 V and 39.5 V) or 300 (24.8 V) shocks. The pulse vector was inverted in this experiment. c) Histogram for the 2017-09-28 experiment averaging $200(15.5 \mathrm{~V}$ and $39.5 \mathrm{~V})$ or $400(24.8 \mathrm{~V})$ shocks. For each of the histograms the dashed line indicates the mean value. 
higher probability with Pinacidil. At the same time no large difference is seen without a shock. This is because without a shock the activation probability directly follows from the activation cycle length (compare Tab. 5.3 and Fig. 5.6) and the change of the cycle length caused by Pinacidil is not very large in absolute numbers.

From this data, it can be deduced that the probability for a shock to excite a certain area is increased due to Pinacidil. The effect of Pinacidil is most likely linked to the excitable gap, since an effect on the recruitment should have been seen in the activation map analysis in Section 5.5. 



\section{Chapter 9}

\section{Summary and Discussion}

In this thesis the complex patterns during ventricular fibrillation were investigated with the aim to better understand and characterize them. One of the central ideas was understanding changes in the arrhythmia with respect to ischemic conditions. Although defibrillation strategies were not studied in detail in this work, it was motivated by the observed variations in the defibrillation success in previous experiments. To understand such differences in defibrillation results, it is necessary to characterize the dynamics and the effect of electrical shocks in detail. For this, experiments were performed with the aim of modifying the dynamics of the arrhythmia using the drug Pinacidil. The effect of the drug is similar to the effects of ischemia, which could be one factor for the observed variations in defibrillation success.

The results of this thesis thus are grouped in the following way:

- Chapter 5 introduces and gives an overview of the Pinacidil experimental series as a basis for the further analysis. The experimentally found changes in dynamical properties during fibrillation are presented in this chapter.

- The following Chapter continues with this analysis by introducing a stochastic model for the investigation of fibrillation and showing how it can help to analyze such data.

- Chapter 6 then focuses on the observation of spatial structuring in the phase singularities. This spatial structuring is related to heterogeneity in the dynamical properties, one cause of which can be ischemia.

- Chapter 7 then shows and discusses methods to allow a better understanding of how shocks affect arrhythmia.

\section{Fibrillation under the Influence of Pinacidil}

In Chapter 5, the datasets used for most of the results in this thesis are described. Further, the data is studied with respect to:

- Properties of activation by electrical shocks using activation time analysis. 
- Properties of the arrhythmia analyzing features such as the cycle length and the number of phase singularities.

The main result is the comparison and characterization of how Pinacidil affects these properties. The chapter concludes that Pinacidil has no effect on the activation time analysis and thus wave recruitment. The results further show Pinacidil affects the dynamical properties of the arrhythmia. More phase singularities are created in its presence and the frequency of activation is higher. This helps to understand how Pinacidil affects arrhythmia and establishes the experimental basis for the stochastic model in Chapter 6.

\section{Characterization of Ventricular Fibrillation using a Markov Model}

Chapter 6 shows the application of a Markov model approach to describe the number of phase singularities during fibrillation. To my knowledge this is the first such application to experimentally observed phase singularities. This model has been used to study turbulent systems and - from simulation studies - can be expected to help to characterize the dynamical properties of the fibrillation. The number of phase singularities are described by using stochastic transition probabilities or rates based on the dynamics of phase singularities: pair creation, pair annihilation, entering from a boundary, and leaving or colliding with a boundary. These rates are described and fitted as functions that depend on the current number of phase singularities.

The analysis presented shows that such a stochastic model is indeed capable of capturing many observations, such as the probability distribution of the number of phase singularities. Further, the properties of the model are discussed in detail since simulation studies suggest that an interpretation of the model parameters - the phase singularity rates of change - may provide information about the dynamical properties of the fibrillation. As an example that these are indeed descriptive, it is shown that the rate of phase singularity creation may have more descriptive power over the changes induced by Pinacidil than the number of phase singularities or the frequency of the arrhythmia which were calculated in Chapter 5. Additionally, I outline that the description using such a model may describe the probability of an arrhythmia ending spontaneously and could help to investigate temporal fluctuations by observing changes in the phase singularity rates of change.

\section{Spatial Organization of Phase Singularities}

The spatial distribution of phase singularities with respect to spatial heterogeneity is discussed in Chapter 7. The chapter includes my publication [106], which uses a model of an excitable medium to showcase how dynamical heterogeneity can affect chaotic spiral wave dynamics. In the extreme case, a full stabilization can occur. This stabilization and the properties of phase singularity reorganization due to the heterogeneity are discussed.

In the numerical study, the importance of local properties with respect to the spatial organization of phase singularities is highlighted. The chapter continues with 
an experimental validation of these observations. The results shown in this thesis give strong evidence, validated by statistical tests, that the two hypotheses are true:

1. Spatial phase singularity organization is stronger when the dynamical properties are more heterogeneous.

2. Dynamical heterogeneities and the phase singularity organization are correlated.

This analysis is shown based on the observations of the numerical study and by characterizing the action potential shape observed in experiments.

\section{Control using Far Field Shocks}

In an exemplary fashion, Chapter 8 discusses how electrical shocks - such as those given during defibrillation - affect ventricular fibrillation. This is achieved by statistically analyzing many shock events and then inspecting the probability of activation due to a shock. The chapter discusses three main points. First, it is seen that activation due to a shock during an arrhythmia indeed appears to occur in a probabilistic manner but in a similar fashion to the quiescent heart. Second, different pacing strategies and wave propagation after the shock are briefly discussed. Third, it highlights that the probability of such activation - or recruitment - is a property of the dynamics which is influenced by Pinacidil.

In this the chapter another important change of the dynamical properties due to Pinacidil is shown. Further, it is outlined that the recruitment during the electrical shock can be visualized and thus analyzed.

\subsection{Discussion and Outlook}

The results summarized above approach the problem of fibrillation and defibrillation from various sides. While defibrillation itself was not discussed in depth in this thesis, detailed understanding of the dynamical properties of the fibrillation is a prerequisite for the better understanding of defibrillation techniques.

Towards this goal, differences in the arrhythmic dynamics need to be understood and quantified and this was studied for the Pinacidil model. In particular the method of using a stochastic model to derive new tools for the investigation of fibrillation was used. In the cardiac context, lifetimes of phase singularities or waves are commonly reported (e.g. [37, 118, 119]), and to some degree, also the frequency of occurrence of the number of phase singularities (e.g. [120]). Chen et al. [43] have also counted the entering-to-leaving ratio of wavelets - into the camera's field of view - for short segments of ventricular fibrillation and argued that a typically higher number of leaving events indicates that the arrhythmia is driven from outside the camera's field of view. Further methods for characterization of ventricular fibrillation such as the mean area swept out by a wave, the multiplicity of the rhythm, or wavefront fractionations and collisions have been proposed and used, many of which were developed by Rogers and 
co-workers [121-123]. Also general parameters such as the wave conduction speed can shed light into the dynamics.

However, the tools previously developed to describe turbulent systems and the Belousov-Zhabotinsky reaction [67-69, 73] have not been applied to cardiac data. These model the number of phase singularities as a stochastic process and, unlike many other measures, were used in theoretical studies. The data presented in chapter 6 shows that such a model also performs well for the heart. With respect to defibrillation, it can be interesting to investigate whether differences such as differences in the selftermination probability - which is approachable using this model - affects defibrillation strategies.

The properties of phase singularities as a central concept are further analyzed here both numerically and experimentally with respect to dynamic heterogeneities. Previous studies have linked such spatial organization to vasculature and other geometrical features [102] and observed reentry at heterogeneities [108-110]. Understanding how heterogeneity affects arrhythmias is important, since many diseases, such as infarction, are associated with local changes in the heart tissue. My experimental results demonstrate that the spatial phase singularity organization is indeed related to dynamical heterogeneities.

The last chapter is more directly relevant to the understanding of defibrillation. It discussed the effects of electrical shocks on the heart. Similar methods have been used to study the effect of local pacing [22, 37]. Here, a statistical approach to this visualization was shown to provide information on activation sites and probabilities. The understanding of why and when a given defibrillation approach is more effective than another is elusive and this means of visualization can help provide insights into the differences. The approach may be particularly interesting in the design of new shock vectors where recruitment is of immediate interest.

Finally, I believe that the results from the stochastic model show that new approaches to the experimental analysis of phase singularities or phase filaments can contribute many new insights into fibrillation in the future. It is my hope that these results derived from the study of the phase singularity dynamics will lead to a better understanding of fibrillation and defibrillation. 


\section{References}

[1] Eurostat. Cardiovascular diseases statistics - Statistics Explained. Sept. 2017. URL: https : / / ec . europa . eu / eurostat / statistics - explained / index . php / Cardiovascular_diseases_statistics.

[2] S. V. Pandit and J. Jalife. "Rotors and the Dynamics of Cardiac Fibrillation". In: Circulation Research 112.5 (Mar. 1, 2013), pp. 849-862. DOI: 10.1161/ CIRCRESAHA.111.300158.

[3] A. Karma. "Physics of Cardiac Arrhythmogenesis". In: Annual Review of Condensed Matter Physics 4.1 (Apr. 2013), pp. 313-337. DOI: 10.1146/annurevconmatphys-020911-125112.

[4] S. Luther et al. "Low-energy control of electrical turbulence in the heart". In: Nature 475.7355 (July 14, 2011), pp. 235-239. DOI: 10.1038/nature10216.

[5] M. Valderrábano. "Influence of anisotropic conduction properties in the propagation of the cardiac action potential". In: Progress in Biophysics and Molecular Biology. Gap junction channels: from protein genes to diseases 94.1 (May 1, 2007), pp. 144-168. DOI: 10.1016/j.pbiomolbio.2007.03.014.

[6] A. Mahajan et al. "A Rabbit Ventricular Action Potential Model Replicating Cardiac Dynamics at Rapid Heart Rates". In: Biophysical Journal 94.2 (Jan. 2008), pp. 392-410. DOI: 10.1529/biophysj.106.98160.

[7] A. T. Winfree. The Geometry of Biological Time. Red. by J. E. Marsden, S. Wiggins, and L. Sirovich. Vol. 12. Interdisciplinary Applied Mathematics. New York, NY: Springer New York, 2001.

[8] F. Fenton and E. Cherry. "Models of cardiac cell". In: Scholarpedia 3.8 (2008), p. 1868. DOI: 10.4249 /scholarpedia.1868.

[9] A. O. Grant. "Cardiac Ion Channels". In: Circulation: Arrhythmia and Electrophysiology 2.2 (Apr. 1, 2009), pp. 185-194. DOI: 10.1161/CIRCEP.108.789081.

[10] F. Sachs. "Stretch-Activated Ion Channels: What Are They?" In: Physiology 25.1 (Feb. 1, 2010), pp. 50-56. DOI: 10.1152/physiol.00042.2009.

[11] M. Bär and M. Eiswirth. "Turbulence due to spiral breakup in a continuous excitable medium". In: Physical Review E 48.3 (Sept. 1, 1993), R1635-R1637. DOI: 10.1103/PhysRevE.48.R1635.

[12] S. Alonso, M. Bär, and A. V. Panfilov. "Negative Tension of Scroll Wave Filaments and Turbulence in Three-Dimensional Excitable Media and Application in Cardiac Dynamics". In: Bulletin of Mathematical Biology 75.8 (Aug. 2013), pp. 1351-1376. DOI: 10.1007/s11538-012-9748-7. 
[13] M.-A. Bray, S.-F. Lin, R. R. Aliev, B. J. Roth, and J. P. Wikswo. "Experimental and theoretical analysis of phase singularity dynamics in cardiac tissue". In: Journal of Cardiovascular Electrophysiology 12.6 (2001), pp. 716-722.

[14] F. H. Fenton, E. M. Cherry, H. M. Hastings, and S. J. Evans. "Multiple mechanisms of spiral wave breakup in a model of cardiac electrical activity". In: Chaos: An Interdisciplinary Journal of Nonlinear Science 12.3 (Sept. 1, 2002), pp. 852-892. DOI: $10.1063 / 1.1504242$.

[15] E. B. Bourgeois, H. D. Reeves, G. P. Walcott, and J. M. Rogers. "Panoramic optical mapping shows wavebreak at a consistent anatomical site at the onset of ventricular fibrillation". In: Cardiovascular Research 93.2 (Feb. 1, 2012), pp. 272-279. DOI: $10.1093 /$ cvr/cvr327.

[16] C. H. Luo and Y. Rudy. "A model of the ventricular cardiac action potential. Depolarization, repolarization, and their interaction." In: Circulation Research 68.6 (June 1, 1991), pp. 1501-1526. DOI: 10.1161/01.RES.68.6.1501.

[17] V. G. Fast, S. Rohr, A. M. Gillis, and A. G. Kléber. "Activation of Cardiac Tissue by Extracellular Electrical Shocks: Formation of 'Secondary Sources' at Intercellular Clefts in Monolayers of Cultured Myocytes". In: Circulation Research 82.3 (Feb. 23, 1998), pp. 375-385. DOI: 10.1161/01.RES.82.3.375.

[18] P. Bittihn, M. Hörning, and S. Luther. "Negative Curvature Boundaries as Wave Emitting Sites for the Control of Biological Excitable Media". In: Physical Review Letters 109.11 (Sept. 14, 2012). DOI: 10.1103/PhysRevLett.109.118106.

[19] N. A. Trayanova and L. J. Rantner. "New insights into defibrillation of the heart from realistic simulation studies". In: Europace 16.5 (May 1, 2014), pp. 705-713. DOI: 10.1093/europace/eut330.

[20] P. Bittihn. Complex Structure and Dynamics of the Heart. Springer Theses. Cham: Springer International Publishing, 2015.

[21] A. Pumir, V. Nikolski, M. Hörning, A. Isomura, K. Agladze, K. Yoshikawa, R. Gilmour, E. Bodenschatz, and V. Krinsky. "Wave Emission from Heterogeneities Opens a Way to Controlling Chaos in the Heart". In: Physical Review Letters 99.20 (Nov. 13, 2007), p. 208101. DOI: 10.1103/PhysRevLett.99.208101.

[22] B. H. KenKnight, P. V. Bayly, R. J. Gerstle, D. L. Rollins, P. D. Wolf, W. M. Smith, and R. E. Ideker. "Regional Capture of Fibrillating Ventricular Myocardium: Evidence of an Excitable Gap". In: Circulation Research 77.4 (Oct. 1, 1995), pp. 849-855. DOI: 10.1161/01.RES.77.4.849.

[23] H. Fei, M. S. Hanna, and L. H. Frame. "Assessing the Excitable Gap in Reentry by Resetting: Implications for Tachycardia Termination by Premature Stimuli and Antiarrhythmic Drugs". In: Circulation 94.9 (Nov. 1, 1996), pp. 2268-2277. DOI: 10.1161/01.CIR.94.9.2268.

[24] P. Bittihn, A. Squires, G. Luther, E. Bodenschatz, V. Krinsky, U. Parlitz, and S. Luther. "Phase-resolved analysis of the susceptibility of pinned spiral waves to far-field pacing in a two-dimensional model of excitable media". In: Philosophical Transactions of the Royal Society A: Mathematical, Physical and Engineering Sciences 368.1918 (May 13, 2010), pp. 2221-2236. DOI: $10.1098 /$ rsta.2010.0038. 
[25] E. Carmeliet. "Cardiac Ionic Currents and Acute Ischemia: From Channels to Arrhythmias". In: Physiological Reviews 79.3 (July 1999), pp. 917-1017. DOI: 10.1152/physrev.1999.79.3.917.

[26] I. Martišienè, J. Jurevičius, R. Vosyliūtè, A. Navalinskas, R. Treinys, R. Mačianskienè, R. Benetis, A. Matiukas, and A. M. Pertsov. "Evolution of Action Potential Alternans in Rabbit Heart during Acute Regional Ischemia". In: BioMed Research International 2015 (2015). DOI: 10.1155/2015/951704.

[27] J. Caldwell, F. L. Burton, G. L. Smith, and S. M. Cobbe. "Heterogeneity of Ventricular Fibrillation Dominant Frequency During Global Ischemia in Isolated Rabbit Hearts". In: Journal of Cardiovascular Electrophysiology 18.8 (Aug. 2007), pp. 854-861. DOI: 10.1111/j.1540-8167.2007.00867.x.

[28] R. Mandapati, Y. Asano, W. T. Baxter, R. Gray, J. Davidenko, and J. Jalife. "Quantification of effects of global ischemia on dynamics of ventricular fibrillation in isolated rabbit heart". In: Circulation 98.16 (1998), pp. 1688-1696.

[29] F. X. Witkowski, L. J. Leon, P. A. Penkoske, W. R. Giles, M. L. Spano, W. L. Ditto, and A. T. Winfree. "Spatiotemporal evolution of ventricular fibrillation". In: Nature 392.6671 (Mar. 5, 1998), pp. 78-82. DOI: 10.1038/32170.

[30] A. G. Kléber, M. J. Janse, F. J. Wilms-Schopmann, A. A. Wilde, and R. Coronel. "Changes in conduction velocity during acute ischemia in ventricular myocardium of the isolated porcine heart." In: Circulation 73.1 (Jan. 1, 1986), pp. 189-198. DOI: 10.1161/01.CIR.73.1.189.

[31] C. P. Bradley, R. H. Clayton, M. P. Nash, A. Mourad, M. Hayward, D. J. Paterson, and P. Taggart. "Human Ventricular Fibrillation During Global Ischemia and Reperfusion". In: Circulation: Arrhythmia and Electrophysiology 4.5 (Oct. 1, 2011), pp. 684-691. DOI: 10.1161/CIRCEP.110.961284.

[32] R. M. Bell, M. M. Mocanu, and D. M. Yellon. "Retrograde heart perfusion: The Langendorff technique of isolated heart perfusion". In: Journal of Molecular and Cellular Cardiology 50.6 (June 1, 2011), pp. 940-950. DOI: 10.1016/j.yjmcc.2011. 02.018 .

[33] D. Hornung. "Cardiac Arrhythmia Termination on the Vascular and Organ Scale". PhD thesis. Göttingen: University of Göttingen, Nov. 11, 2014.

[34] A. T. Winfree. "Electrical instability in cardiac muscle: phase singularities and rotors". In: Journal of theoretical Biology 138.3 (1989), pp. 353-405.

[35] J. M. Davidenko, P. F. Kent, D. R. Chialvo, D. C. Michaels, and J. Jalife. "Sustained vortex-like waves in normal isolated ventricular muscle." In: Proceedings of the National Academy of Sciences 87.22 (Nov. 1, 1990), pp. 8785-8789. DOI: $10.1073 /$ pnas.87.22.8785.

[36] J. M. Davidenko, A. V. Pertsov, R. Salomonsz, W. Baxter, and J. Jalife. "Stationary and drifting spiral waves of excitation in isolated cardiac muscle". In: Nature 355.6358 (Jan. 1992), p. 349. DOI: 10.1038/355349a0.

[37] P. V. Bayly, B. H. KenKnight, J. M. Rogers, E. E. Johnson, R. E. Ideker, and W. M. Smith. "Spatial organization, predictability, and determinism in ventricular fibrillation". In: Chaos: An Interdisciplinary Journal of Nonlinear Science 8.1 (Mar. 1998), pp. 103-115. DOI: 10.1063/1.166291. 
[38] R. A. Gray, A. M. Pertsov, and J. Jalife. "Spatial and temporal organization during cardiac fibrillation". In: Nature 392.6671 (Mar. 5, 1998), pp. 75-78. DOI: $10.1038 / 32164$.

[39] I. R. Efimov, V. Sidorov, Y. Cheng, and B. Wollenzier. "Evidence of ThreeDimensional Scroll Waves with Ribbon-Shaped Filament as a Mechanism of Ventricular Tachycardia in the Isolated Rabbit Heart". In: Journal of cardiovascular electrophysiology 10.11 (1999), pp. 1452-1462.

[40] C. Frothingham and G. R. Minot. "Normal temperature of rabbits". In: American Journal of Physiology-Legacy Content 30.6 (Sept. 2, 1912), pp. 430-435. DOI: 10.1152/ajplegacy.1912.30.6.430.

[41] A. D. Bachtel, R. A. Gray, J. M. Stohlman, E. B. Bourgeois, A. E. Pollard, and J. M. Rogers. "A Novel Approach to Dual Excitation Ratiometric Optical Mapping of Cardiac Action Potentials With Di-4-ANEPPS Using Pulsed LED Excitation". In: IEEE Transactions on Biomedical Engineering 58.7 (July 2011), pp. 2120-2126. DOI: 10.1109/TBME.2011.2148719.

[42] J. Christoph, J. Schröder-Schetelig, and S. Luther. "Electromechanical optical mapping". In: Progress in Biophysics and Molecular Biology. Cardiac Mechanics and Electrics: it takes two to tango 130 (Nov. 1, 2017), pp. 150-169. DOI: 10.1016/j.pbiomolbio.2017.09.015.

[43] J. Chen, R. Mandapati, O. Berenfeld, A. C. Skanes, and J. Jalife. "HighFrequency Periodic Sources Underlie Ventricular Fibrillation in the Isolated Rabbit Heart". In: Circulation Research 86.1 (Jan. 7, 2000), pp. 86-93. DOI: 10.1161/01.RES.86.1.86.

[44] K. E. Brack, R. Narang, J. Winter, and G. A. Ng. "The mechanical uncoupler blebbistatin is associated with significant electrophysiological effects in the isolated rabbit heart: Electrophysiological effects associated with blebbistatin". In: Experimental Physiology 98.5 (May 2013), pp. 1009-1027. DOI: 10.1113/ expphysiol.2012.069369.

[45] L. M. Swift, H. Asfour, N. G. Posnack, A. Arutunyan, M. W. Kay, and N. Sarvazyan. "Properties of blebbistatin for cardiac optical mapping and other imaging applications". In: Pflügers Archiv - European Journal of Physiology 464.5 (Nov. 1, 2012), pp. 503-512. DOI: 10.1007/s00424-012-1147-2.

[46] M. Képiró, B. H. Várkuti, L. Végner, G. Vörös, G. Hegyi, M. Varga, and A. Málnási-Csizmadia. "para-Nitroblebbistatin, the Non-Cytotoxic and Photostable Myosin II Inhibitor". In: Angewandte Chemie International Edition 53.31 (July 28, 2014), pp. 8211-8215. DOI: 10.1002/anie.201403540.

[47] B. H. Várkuti, M. Képiró, I. Á. Horváth, L. Végner, S. Ráti, Á. Zsigmond, G. Hegyi, Z. Lenkei, M. Varga, and A. Málnási-Csizmadia. "A highly soluble, non-phototoxic, non-fluorescent blebbistatin derivative". In: Scientific Reports 6 (May 31, 2016), p. 26141. DOI: 10.1038/srep26141.

[48] A. Hassner, D. Birnbaum, and L. M. Loew. "Charge-shift probes of membrane potential. Synthesis". In: The Journal of Organic Chemistry 49.14 (1984), pp. 2546-2551. 
[49] D. Gross, L. M. Loew, and W. W. Webb. "Optical imaging of cell membrane potential changes induced by applied electric fields". In: Biophysical Journal 50.2 (Aug. 1, 1986), pp. 339-348. DOI: 10.1016/S0006-3495(86)83467-1.

[50] A. Y. H. Chen. "Development and characterization of fiber-based systems for biomedical imaging". PhD thesis. ResearchSpace@ Auckland, 2011.

[51] C. J. Hyatt, S. F. Mironov, M. Wellner, O. Berenfeld, A. K. Popp, D. A. Weitz, J. Jalife, and A. M. Pertsov. "Synthesis of Voltage-Sensitive Fluorescence Signals from Three-Dimensional Myocardial Activation Patterns". In: Biophysical Journal 85.4 (Oct. 1, 2003), pp. 2673-2683. DOI: 10.1016/S0006-3495(03)746906.

[52] M. J. Bishop, B. Rodriguez, F. Qu, I. R. Efimov, D. J. Gavaghan, and N. A. Trayanova. "The Role of Photon Scattering in Optical Signal Distortion during Arrhythmia and Defibrillation". In: Biophysical Journal 93.10 (Nov. 2007), pp. 3714-3726. DOI: 10.1529/biophysj.107.110981.

[53] A. Matiukas et al. "Near-infrared voltage-sensitive fluorescent dyes optimized for optical mapping in blood-perfused myocardium". In: Heart Rhythm 4.11 (Nov. 1, 2007), pp. 1441-1451. DOI: 10.1016/j.hrthm.2007.07.012.

[54] D. C. Sigg, P. A. Iaizzo, Y.-F. Xiao, and B. He, eds. Cardiac Electrophysiology Methods and Models. Boston, MA: Springer US, 2010. DOI: 10.1007/978-14419-6658-2.

[55] A. Pumir, S. Sinha, S. Sridhar, M. Argentina, M. Hörning, S. Filippi, C. Cherubini, S. Luther, and V. Krinsky. "Wave-train-induced termination of weakly anchored vortices in excitable media". In: Physical Review E 81.1 (Jan. 11, 2010), p. 010901. DOI: 10.1103/PhysRevE.81.010901.

[56] M. Tanaka, A. Isomura, M. Hörning, H. Kitahata, K. Agladze, and K. Yoshikawa. "Unpinning of a spiral wave anchored around a circular obstacle by an external wave train: Common aspects of a chemical reaction and cardiomyocyte tissue". In: Chaos: An Interdisciplinary Journal of Nonlinear Science 19.4 (2009), p. 043114. DOI: $10.1063 / 1.3263167$.

[57] M. Hörning. "Termination of pinned vortices by high-frequency wave trains in heartlike excitable media with anisotropic fiber orientation". In: Physical Review E 86.3 (Sept. 14, 2012). DOI: 10.1103/PhysRevE.86.031912.

[58] Y. C. Ji, I. Uzelac, N. Otani, S. Luther, R. F. Gilmour, E. M. Cherry, and F. H. Fenton. "Synchronization as a mechanism for low-energy anti-fibrillation pacing". In: Heart Rhythm 14.8 (Aug. 2017), pp. 1254-1262. DOI: 10.1016/j. hrthm.2017.05.021.

[59] A. Behrend, P. Bittihn, and S. Luther. "Predicting unpinning success rates for a pinned spiral in an excitable medium". In: 2010 Computing in Cardiology. 2010 Computing in Cardiology. Sept. 2010, pp. 345-348.

[60] T. K. Shajahan, S. Berg, S. Luther, V. Krinski, and P. Bittihn. "Scanning and resetting the phase of a pinned spiral wave using periodic far field pulses". In: New Journal of Physics 18.4 (2016), p. 043012. DOI: 10.1088/1367-2630/18/4/ 043012. 
[61] D. Hornung, V. N. Biktashev, N. F. Otani, T. K. Shajahan, T. Baig, S. Berg, S. Han, V. I. Krinsky, and S. Luther. "Mechanisms of vortices termination in the cardiac muscle". In: Royal Society Open Science 4.3 (Mar. 1, 2017), p. 170024. DOI: $10.1098 /$ rsos. 170024 .

[62] A. S. Tang, S. Yabe, J. M. Wharton, M. Dolker, W. M. Smith, and R. E. Ideker. "Ventricular defibrillation using biphasic waveforms: the importance of phasic duration". In: Journal of the American College of Cardiology 13.1 (1989), pp. 207-214.

[63] A. Pumir and V. Krinsky. "Unpinning of a Rotating Wave in Cardiac Muscle by an Electric Field". In: Journal of Theoretical Biology 199.3 (Aug. 7, 1999), pp. 311-319. DOI: 10.1006/jtbi.1999.0957.

[64] P. Coullet, L. Gil, and J. Lega. "Defect-mediated turbulence". In: Physical Review Letters 62.14 (Apr. 3, 1989), pp. 1619-1622. DOI: 10.1103/PhysRevLett. 62.1619 .

[65] L. Gil, J. Lega, and J. L. Meunier. "Statistical properties of defect-mediated turbulence". In: Physical Review A 41.2 (1990), p. 1138.

[66] J. Lega. "Defect-mediated turbulence". In: Computer Methods in Applied Mechanics and Engineering. Second World Congress on Computational Mechanics 89.1 (Aug. 1, 1991), pp. 419-424. DOI: 10.1016/0045-7825(91)90051-7.

[67] K. E. Daniels and E. Bodenschatz. "Defect Turbulence in Inclined Layer Convection". In: Physical Review Letters 88.3 (Jan. 7, 2002), p. 034501. DOI: 10. 1103/PhysRevLett.88.034501.

[68] Y.-N. Young and H. Riecke. "Penta-Hepta Defect Chaos in a Model for Rotating Hexagonal Convection". In: Physical Review Letters 90.13 (Apr. 3, 2003). DOI: 10.1103/PhysRevLett.90.134502.

[69] K. E. Daniels, C. Beck, and E. Bodenschatz. "Defect turbulence and generalized statistical mechanics". In: Physica D: Nonlinear Phenomena. Anomalous distributions, nonlinear dynamics, and nonextensivity 193.1 (June 15, 2004), pp. 208-217. DOI: 10.1016/j.physd.2004.01.033.

[70] C. Huepe, H. Riecke, K. E. Daniels, and E. Bodenschatz. "Statistics of defect trajectories in spatio-temporal chaos in inclined layer convection and the complex Ginzburg-Landau equation". In: Chaos: An Interdisciplinary Journal of Nonlinear Science 14.3 (Sept. 1, 2004), pp. 864-874. DOI: 10.1063/1.1778495.

[71] J. Davidsen, A. Mikhailov, and R. Kapral. "Front explosion in a periodically forced surface reaction". In: Physical Review E 72.4 (Oct. 20, 2005). DOI: 10. 1103/PhysRevE.72.046214.

[72] J. Davidsen, M. Zhan, and R. Kapral. "Filament-Induced Surface Spiral Turbulence in Three-Dimensional Excitable Media". In: Physical Review Letters 101.20 (Nov. 11, 2008), p. 208302. DOI: 10.1103/PhysRevLett.101.208302.

[73] C. Qiao, H. Wang, and Q. Ouyang. "Defect-mediated turbulence in the Belousov-Zhabotinsky reaction". In: Physical Review E 79.1 (Jan. 21, 2009). DOI: 10.1103/PhysRevE.79.016212. 
[74] G. St-Yves and J. Davidsen. "Influence of the medium's dimensionality on defect-mediated turbulence". In: Physical Review E 91.3 (Mar. 26, 2015). DOI: 10.1103/PhysRevE.91.032926.

[75] D. T. Gillespie. "A general method for numerically simulating the stochastic time evolution of coupled chemical reactions". In: Journal of Computational Physics 22.4 (Dec. 1, 1976), pp. 403-434. DOI: 10.1016/0021-9991(76)90041-3.

[76] K. Sugimura and H. Kori. "Exponential system-size dependence of the lifetime of transient spiral chaos in excitable and oscillatory media". In: Physical Review E 92.6 (2015), p. 062915.

[77] M. C. Strain and H. S. Greenside. "Size-dependent transition to highdimensional chaotic dynamics in a two-dimensional excitable medium". In: Physical Review Letters 80.11 (1998), p. 2306.

[78] C. Beta, A. S. Mikhailov, H. H. Rotermund, and G. Ertl. "Defect-mediated turbulence in a catalytic surface reaction". In: Europhysics Letters (EPL) 75.6 (Sept. 2006), pp. 868-874. DOI: 10.1209/epl/i2006-10215-5.

[79] C. Beta. "Controlling chemical turbulence in surface reactions". PhD thesis. Berlin, Germany: Freie Universität Berlin, Jan. 18, 2005.

[80] M. Hildebrand, M. Bär, and M. Eiswirth. "Statistics of Topological Defects and Spatiotemporal Chaos in a Reaction-Diffusion System". In: Physical Review Letters 75.8 (Aug. 21, 1995), pp. 1503-1506. DOI: 10.1103/PhysRevLett.75. 1503.

[81] J. I. Laughner, F. S. Ng, M. S. Sulkin, R. M. Arthur, and I. R. Efimov. "Processing and analysis of cardiac optical mapping data obtained with potentiometric dyes". In: American Journal of Physiology-Heart and Circulatory Physiology 303.7 (July 20, 2012), H753-H765. DOI: 10.1152/ajpheart.00404.2012.

[82] K. Wang, P. Lee, G. R. Mirams, P. Sarathchandra, T. K. Borg, D. J. Gavaghan, P. Kohl, and C. Bollensdorff. "Cardiac tissue slices: preparation, handling, and successful optical mapping". In: American Journal of Physiology - Heart and Circulatory Physiology 308.9 (May 1, 2015), H1112-H1125. DOI: 10.1152/ajpheart.00556.2014.

[83] A. Schlemmer, S. Berg, T. Shajahan, S. Luther, and U. Parlitz. "Entropy Rate Maps of Complex Excitable Dynamics in Cardiac Monolayers". In: Entropy 17.3 (Feb. 26, 2015), pp. 950-967. DOI: 10.3390/e17030950.

[84] J. Canny. "A Computational Approach to Edge Detection". In: IEEE Transactions on Pattern Analysis and Machine Intelligence PAMI-8.6 (Nov. 1986), pp. 679-698. DOI: 10.1109/TPAMI.1986.4767851.

[85] A. Schlemmer, S. Berg, T. Lilienkamp, S. Luther, and U. Parlitz. "Spatiotemporal Permutation Entropy as a Measure for Complexity of Cardiac Arrhythmia". In: Frontiers in Physics 6 (2018). DOI: 10.3389/fphy.2018.00039.

[86] E. Jones, T. Oliphant, P. Peterson, et al. SciPy: Open source scientific tools for Python. 2001.

[87] L. M. Hondeghem, L. Carlsson, and G. Duker. "Instability and Triangulation of the Action Potential Predict Serious Proarrhythmia, but Action Potential Duration Prolongation Is Antiarrhythmic". In: Circulation (Apr. 17, 2001). 
[88] Y.-B. Liu, A. Peter, S. T. Lamp, J. N. Weiss, P.-S. Chen, and S.-F. Lin. "Spatiotemporal Correlation Between Phase Singularities and Wavebreaks During Ventricular Fibrillation". In: Journal of Cardiovascular Electrophysiology 14.10 (Oct. 2003), pp. 1103-1109. DOI: 10.1046/j.1540-8167.2003.03218.x.

[89] H. Kantz and T. Schreiber. Nonlinear Time Series Analysis. Cambridge University Press, 2004.

[90] W. E. Lorensen and H. E. Cline. "Marching cubes: A high resolution 3D surface construction algorithm". In: Computer Graphics. Vol. 21. Anaheim, California: ACM, July 27, 1987, pp. 163-169.

[91] S. van der Walt, J. L. Schönberger, J. Nunez-Iglesias, F. Boulogne, J. D. Warner, N. Yager, E. Gouillart, and T. Yu. "scikit-image: image processing in Python". In: PeerJ 2 (June 19, 2014), e453. DOI: 10.7717/peerj.453.

[92] M. W. Kay, G. P. Walcott, J. D. Gladden, S. B. Melnick, and J. M. Rogers. "Lifetimes of epicardial rotors in panoramic optical maps of fibrillating swine ventricles". In: AJP: Heart and Circulatory Physiology 291.4 (June 2, 2006), H1935-H1941. DOI: 10.1152/ajpheart.00276.2006.

[93] B. Trénor, J. M. Ferrero, B. Rodríguez, and F. Montilla. "Effects of Pinacidil on Reentrant Arrhythmias Generated During Acute Regional Ischemia: A Simulation Study". In: Annals of Biomedical Engineering 33.7 (July 2005), pp. 897906. DOI: $10.1007 / \mathrm{s} 10439-005-3554-4$.

[94] S. Wu, H. Hayashi, S.-F. Lin, and P.-S. Chen. "Action Potential Duration and QT Interval During Pinacidil Infusion in Isolated Rabbit Hearts". In: Journal of Cardiovascular Electrophysiology 16.8 (Aug. 1, 2005), pp. 872-878. DOI: 10. 1111/j.1540-8167.2005.40811.x.

[95] V. V. Fedorov, A. V. Glukhov, C. M. Ambrosi, G. Kostecki, R. Chang, D. Janks, R. B. Schuessler, N. Moazami, C. G. Nichols, and I. R. Efimov. "Effects of KATP channel openers diazoxide and pinacidil in coronary-perfused atria and ventricles from failing and non-failing human hearts". In: Journal of Molecular and Cellular Cardiology 51.2 (Aug. 1, 2011), pp. 215-225. DOI: 10.1016/j.yjmcc. 2011.04.016.

[96] J. G. Quintanilla et al. "KATP channel opening accelerates and stabilizes rotors in a swine heart model of ventricular fibrillation". In: Cardiovascular Research 99.3 (Aug. 1, 2013), pp. 576-585. DOI: 10.1093/cvr/cvt093.

[97] T. Hastie, R. Tibshirani, and J. Friedman. The Elements of Statistical Learning: Data Mining, Inference, and Prediction, Second Edition. 2nd ed. Springer Series in Statistics. New York: Springer-Verlag, 2009.

[98] R. Durbin, S. R. Eddy, A. Krogh, and G. Mitchison. Biological sequence analysis: Probabilistic models of proteins and nucleic acids. Cambridge: Cambridge University Press, 1998. DOI: 10.1017/CBO9780511790492.

[99] S. Alonso, F. Sagués, and A. S. Mikhailov. "Taming Winfree Turbulence of Scroll Waves in Excitable Media”. In: Science 299.5613 (Mar. 14, 2003), pp. 1722-1725. DOI: 10.1126/science.1080207.

[100] C. Gardiner. Stochastic Methods: A Handbook for the Natural and Social Sciences. Springer Berlin Heidelberg, Oct. 19, 2010. 
[101] A. Schlemmer. "Intermittent Complexity Fluctuations during Ventricular Fibrillation". PhD thesis. Göttingen: Georg-August-Universität Göttingen, 2017.

[102] M. Valderrábano, P.-S. Chen, and S.-F. Lin. "Spatial Distribution of Phase Singularities in Ventricular Fibrillation". In: Circulation 108.3 (July 22, 2003), pp. 354-359. DOI: 10.1161/01.CIR.0000080322.67408.B4.

[103] R. H. Keldermann, K. H. W. J. ten Tusscher, M. P. Nash, R. Hren, P. Taggart, and A. V. Panfilov. "Effect of heterogeneous APD restitution on VF organization in a model of the human ventricles". In: American Journal of Physiology-Heart and Circulatory Physiology 294.2 (Feb. 1, 2008), H764-H774. DOI: 10.1152/ajpheart.00906.2007.

[104] F. Spreckelsen, D. Hornung, O. Steinbock, U. Parlitz, and S. Luther. "Stabilization of three-dimensional scroll waves and suppression of spatiotemporal chaos by heterogeneities". In: Physical Review E 92.4 (Oct. 22, 2015), p. 042920. DOI: 10.1103/PhysRevE.92.042920.

[105] V. Y. Sidorov, I. Uzelac, and J. P. Wikswo. "Regional increase of extracellular potassium leads to electrical instability and reentry occurrence through the spatial heterogeneity of APD restitution". In: AJP: Heart and Circulatory Physiology 301.1 (July 1, 2011), H209-H220. DOI: 10.1152/ajpheart.01141.2010.

[106] P. Bittihn, S. Berg, U. Parlitz, and S. Luther. "Emergent dynamics of spatiotemporal chaos in a heterogeneous excitable medium". In: Chaos: An Interdisciplinary Journal of Nonlinear Science 27.9 (Sept. 1, 2017), p. 093931. DOI: 10.1063/1.4999604.

[107] R. C. Team. R: A language and environment for statistical computing. Vienna, Austria, 2018.

[108] A. V. Zaitsev, P. K. Guha, F. Sarmast, A. Kolli, O. Berenfeld, A. M. Pertsov, J. R. d. Groot, R. Coronel, and J. Jalife. "Wavebreak Formation During Ventricular Fibrillation in the Isolated, Regionally Ischemic Pig Heart". In: Circulation Research (Mar. 21, 2003).

[109] K. Tanaka et al. "Spatial Distribution of Fibrosis Governs Fibrillation Wave Dynamics in the Posterior Left Atrium During Heart Failure". In: Circulation Research 101.8 (Oct. 12, 2007), pp. 839-847. DOI: 10.1161/CIRCRESAHA.107. 153858.

[110] M. Vaquero, D. Calvo, and J. Jalife. "Cardiac fibrillation: From ion channels to rotors in the human heart". In: Heart Rhythm 5.6 (June 2008), pp. 872-879. DOI: $10.1016 /$ j.hrthm.2008.02.034.

[111] A. L. Wit, S. M. Dillon, J. Coromilas, A. E. Saltman, and B. Waldecker. "Anisotropic Reentry in the Epicardial Border Zone of Myocardial Infarctsa". In: Annals of the New York Academy of Sciences 591.1 (June 1, 1990), pp. 86108. DOI: $10.1111 /$ j.1749-6632.1990.tb15083.x.

[112] P. Comtois and S. Nattel. "Impact of tissue geometry on simulated cholinergic atrial fibrillation: A modeling study". In: Chaos: An Interdisciplinary Journal of Nonlinear Science 21.1 (Mar. 2011), p. 013108. DOI: 10.1063/1.3544470. 
[113] A. Defauw, N. Vandersickel, P. Dawyndt, and A. V. Panfilov. "Small size ionic heterogeneities in the human heart can attract rotors". In: American Journal of Physiology - Heart and Circulatory Physiology 307.10 (Nov. 15, 2014), H1456H1468. DOI: 10.1152/ajpheart.00410.2014.

[114] K. Wheeler, V. Krinski, and N. Otani. "A New, Low-Energy Defibrillation Strategy: Use of Multiple Electric Field Directions to Reshape Scroll Wave Filaments". In: 2017 Computing in Cardiology Conference. Sept. 14, 2017. DOI: 10.22489/CinC.2017.230-449.

[115] L. J. Rantner, B. M. Tice, and N. A. Trayanova. "Terminating ventricular tachyarrhythmias using far-field low-voltage stimuli: Mechanisms and delivery protocols". In: Heart Rhythm 10.8 (Aug. 2013), pp. 1209-1217. DOI: 10.1016/j. hrthm.2013.04.027.

[116] P. Buran, M. Bär, S. Alonso, and T. Niedermayer. "Control of electrical turbulence by periodic excitation of cardiac tissue". In: Chaos: An Interdisciplinary Journal of Nonlinear Science 27.11 (Nov. 2017), p. 113110. DOI: 10.1063/1. 5010787.

[117] P. D. Bella, G. Marenzi, C. Tondo, D. Cardinale, F. Giraldi, G. Lauri, and M. Guazzi. "Usefulness of excitable gap and pattern of resetting in atrial flutter for determining reentry circuit location". In: The American Journal of Cardiology 68.5 (Aug. 15, 1991), pp. 492-497. DOI: 10.1016/0002-9149(91)90784-I.

[118] J. M. Rogers, J. Huang, W. M. Smith, and R. E. Ideker. "Incidence, Evolution, and Spatial Distribution of Functional Reentry During Ventricular Fibrillation in Pigs". In: Circulation Research 84.8 (Apr. 30, 1999), pp. 945-954. DOI: 10. 1161/01.RES.84.8.945.

[119] J. Christoph et al. "Electromechanical vortex filaments during cardiac fibrillation". In: Nature 555.7698 (Mar. 2018), pp. 667-672. DOI: 10.1038/nature26001.

[120] M. P. Nash, A. Mourad, R. H. Clayton, P. M. Sutton, C. P. Bradley, M. Hayward, D. J. Paterson, and P. Taggart. "Evidence for Multiple Mechanisms in Human Ventricular Fibrillation". In: Circulation 114.6 (Aug. 8, 2006), pp. 536542. DOI: 10.1161/CIRCULATIONAHA.105.602870.

[121] J. M. Rogers, M. Usui, B. H. KenKnight, R. E. Ideker, and W. M. Smith. "Recurrent wavefront morphologies: A method for quantifying the complexity of epicardial activation patterns". In: Annals of Biomedical Engineering 25.5 (Sept. 1, 1997), pp. 761-768. DOI: 10.1007/BF02684160.

[122] J. Huang, J. M. Rogers, C. R. Killingsworth, K. P. Singh, W. M. Smith, and R. E. Ideker. "Evolution of activation patterns during long-duration ventricular fibrillation in dogs". In: American Journal of Physiology - Heart and Circulatory Physiology 286.3 (Mar. 1, 2004), H1193-H1200. DOI: 10.1152/ajpheart.00773. 2003.

[123] K.-A. Cheng, D. J. Dosdall, L. Li, J. M. Rogers, R. E. Ideker, and J. Huang. "Evolution of activation patterns during long-duration ventricular fibrillation in pigs". In: American Journal of Physiology - Heart and Circulatory Physiology 302.4 (Feb. 15, 2012), H992-H1002. DOI: 10.1152/ajpheart.00419.2011. 
[124] J. M. Rogers. "Combined phase singularity and wavefront analysis for optical maps of ventricular fibrillation". In: IEEE Transactions on Biomedical Engineering 51.1 (Jan. 2004), pp. 56-65. DOI: 10.1109/TBME.2003.820341.

[125] A. M. Climent et al. "Role of atrial tissue remodeling on rotor dynamics: an in vitro study". In: American Journal of Physiology - Heart and Circulatory Physiology 309.11 (Dec. 1, 2015), H1964-H1973. DOI: 10.1152/ajpheart.00055. 2015.

[126] F. Fenton and A. Karma. "Vortex dynamics in three-dimensional continuous myocardium with fiber rotation: Filament instability and fibrillation". In: Chaos: An Interdisciplinary Journal of Nonlinear Science 8.1 (Mar. 1, 1998), pp. 20-47. DOI: $10.1063 / 1.166311$.

[127] A. N. Iyer and R. A. Gray. "An Experimentalist's Approach to Accurate Localization of Phase Singularities during Reentry". In: Annals of Biomedical Engineering 29.1 (Jan. 2001), pp. 47-59. DOI: 10.1114/1.1335538.

[128] N. E. Huang, Z. Shen, S. R. Long, M. C. Wu, H. H. Shih, Q. Zheng, N.-C. Yen, C. C. Tung, and H. H. Liu. "The empirical mode decomposition and the Hilbert spectrum for nonlinear and non-stationary time series analysis". In: Proceedings of the Royal Society of London A: Mathematical, Physical and Engineering Sciences 454.1971 (Mar. 8, 1998), pp. 903-995. DOI: 10.1098/rspa.1998.0193.

[129] M.-A. Bray and J. P. Wikswo. "Considerations in phase plane analysis for nonstationary reentrant cardiac behavior". In: Physical Review E 65.5 (May 2, 2002). DOI: 10.1103/PhysRevE.65.051902.

[130] N. Tomii, M. Yamazaki, T. Arafune, H. Honjo, N. Shibata, and I. Sakuma. "Detection Algorithm of Phase Singularity Using Phase Variance Analysis for Epicardial Optical Mapping Data". In: IEEE Transactions on Biomedical Engineering 63.9 (Sept. 2016), pp. 1795-1803. DOI: 10.1109/TBME.2015.2502726.

[131] Y.-S. Lee, J.-S. Song, M. Hwang, B. Lim, B. Joung, and H.-N. Pak. "A New Efficient Method for Detecting Phase Singularity in Cardiac Fibrillation". In: PLoS ONE 11.12 (Dec. 1, 2016). Ed. by T. Ai, e0167567. DOI: 10.1371/journal. pone.0167567. 



\section{Appendix A}

\section{Pinacidil Experiments}

The following table lists the experimenters for each experiment of the series. Two previous experiments performed mainly by me are not included. More experiments which were performed later are not listed since they could not be included in the analysis anymore. Marion Kunze helped in many of the experiments with the first steps of experiment and heart preparation.

Table A.1 Overview over experimenter who performed the Pinacidil experiments.

Experiment Day Experimentators

2017-09-21 Sebastian Berg, Alexander Schlemmer

2017-09-28 Sebastian Berg, Daniel Hornung

2017-10-05 Sebastian Berg, Alexander Schlemmer, Daniel Hornung

2017-10-12 Henrik tom Wörden, Sebastian Berg, Daniel Hornung

2017-10-17 Sebastian Berg, Daniel Hornung, Alexander Schlemmer

2017-11-02 Henrik tom Wörden, Daniel Hornung, Sebastian Berg

2017-11-16 Sebastian Berg, Henrik tom Wörden, Daniel Hornung

2017-11-23 Sebastian Berg, Daniel Hornung

2017-11-30 Henrik tom Wörden, Daniel Hornung

2018-03-28 Henrik tom Wörden, Daniel Hornung 


\section{A.1 Overview Plots for all Experiments}

The following lists overviews for all Pinacidil experiments. These plots include the triangularity index, action potential duration (APD) as measured during the Experiments. Note that both triangularity index and APD drop after a VF episode and then relax slowly, which causes the short time drops. Additional markers give specific times when the Pinacidil concentration was changed or VF would spontaneously induce. Further, the flow rate through the heart (as manually measured) as well as the pressure are shown. This plot additionally marks the time when the heart was excised as well as when Blebbistatin was given. Furthermore, markers show when activation maps were measured. Also restitution curve measurements are marked, although not shown in depth in this thesis. With the exception of pressure, in all plots lines are only drawn to guide the eye. Note that the flow rate is measured manually. The triangularity and APD as shown here are calculated from the PseudoECG (average fluorescent signal) of the front right camera (pointing towards the LV). The analysis was performed during the sinus rhythm.

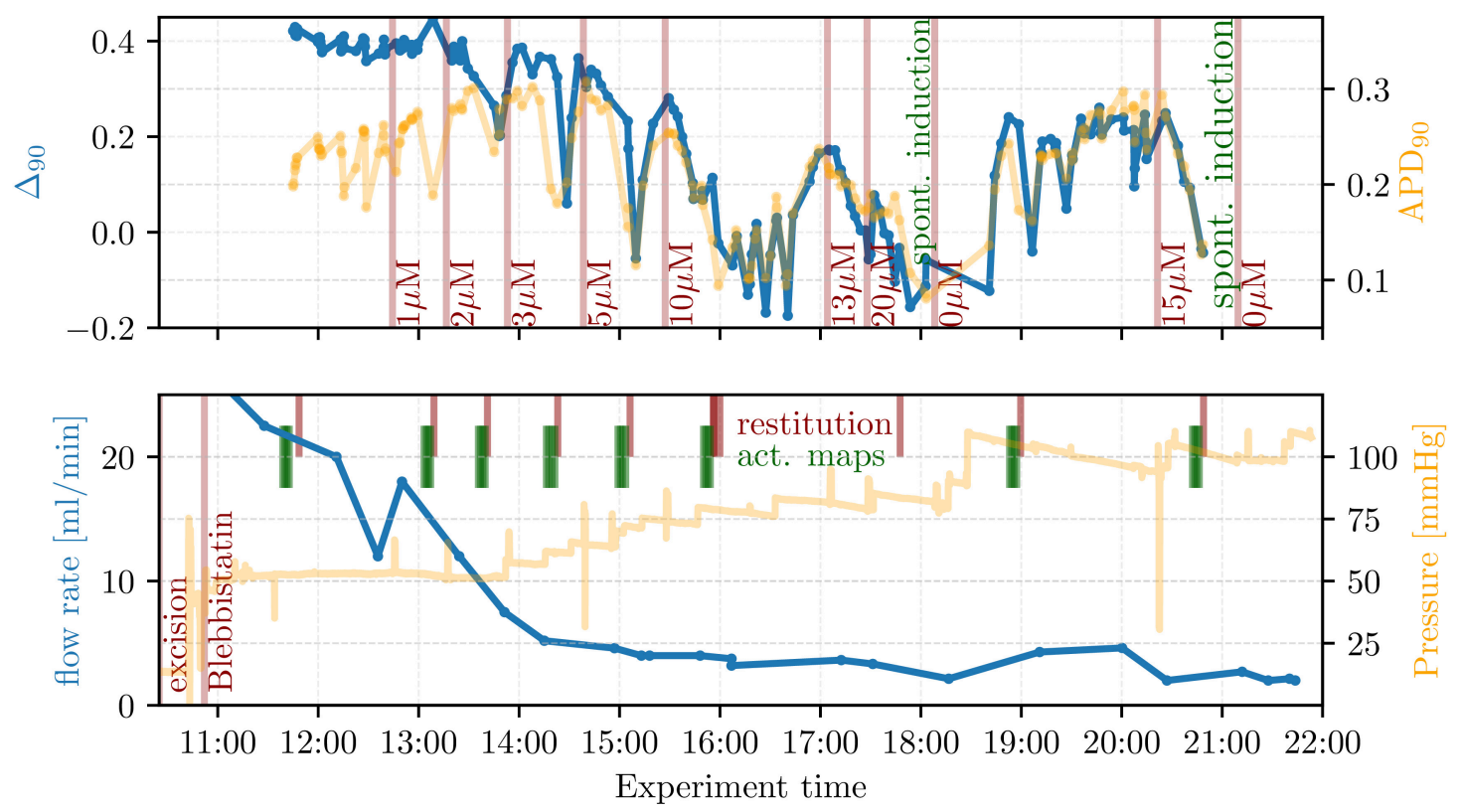

Fig. A.1 Overview plot summarizing the 2017-09-21 Experiment. The plot shows the triangularity index, APD as well as flow rate and pressure over time. Important times such as the change in Pinacidil concentration (red lines, top panel as $\mu \mathrm{M}$ ) are indicated. 

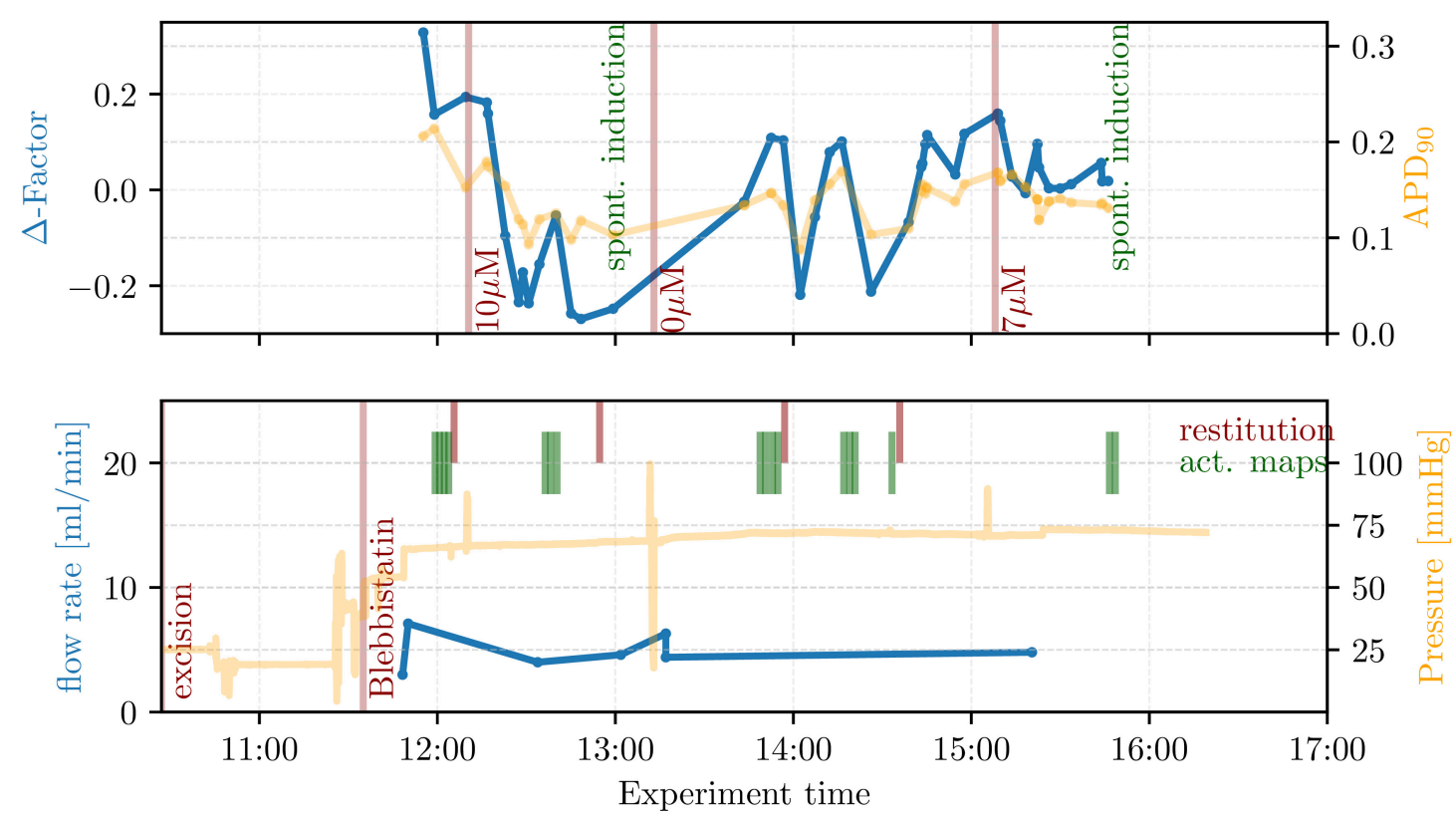

Fig. A.2 Overview plot summarizing the 2017-09-28 Experiment. The plot shows the triangularity index, APD as well as flow rate and pressure over time. Important times such as the change in Pinacidil concentration (red lines, top panel as $\mu \mathrm{M}$ ) are indicated.
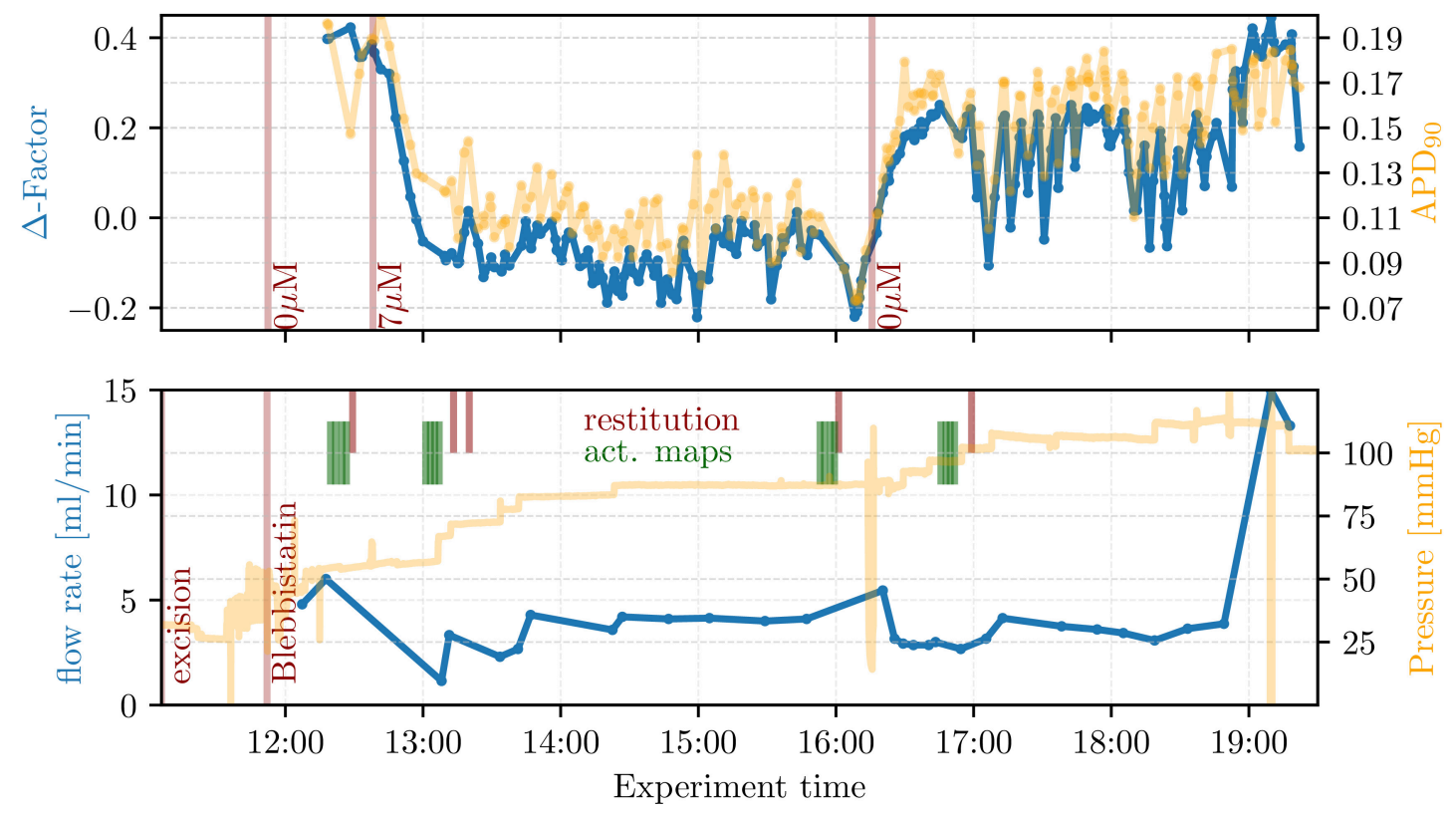

Fig. A.3 Overview plot summarizing the 2017-10-05 Experiment. The plot shows the triangularity index, APD as well as flow rate and pressure over time. Important times such as the change in Pinacidil concentration (red lines, top panel as $\mu \mathrm{M}$ ) are indicated. 

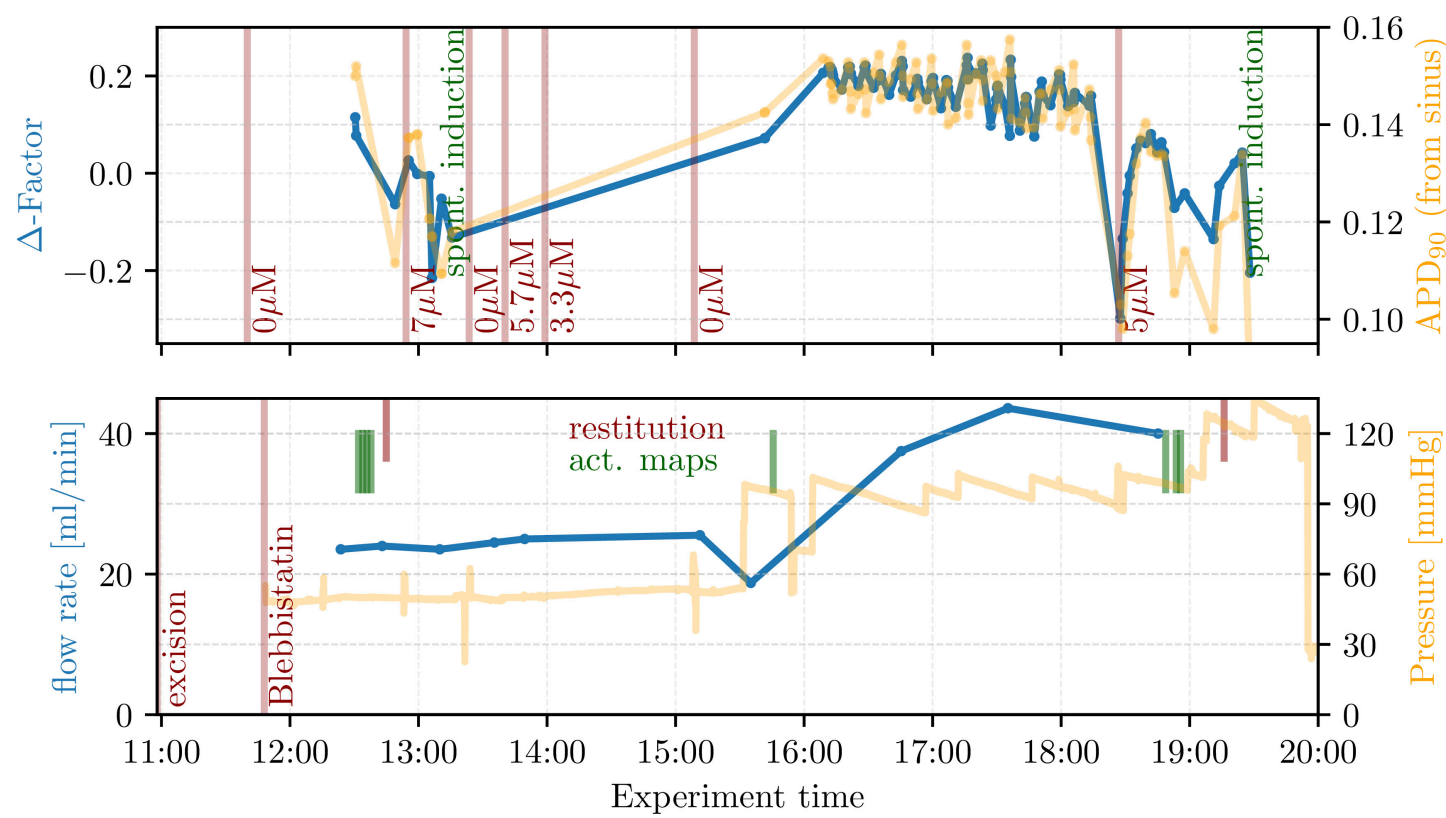

Fig. A.4 Overview plot summarizing the 2017-10-12 Experiment. The plot shows the triangularity index, APD as well as flow rate and pressure over time. Important times such as the change in Pinacidil concentration (red lines, top panel as $\mu \mathrm{M}$ ) are indicated.
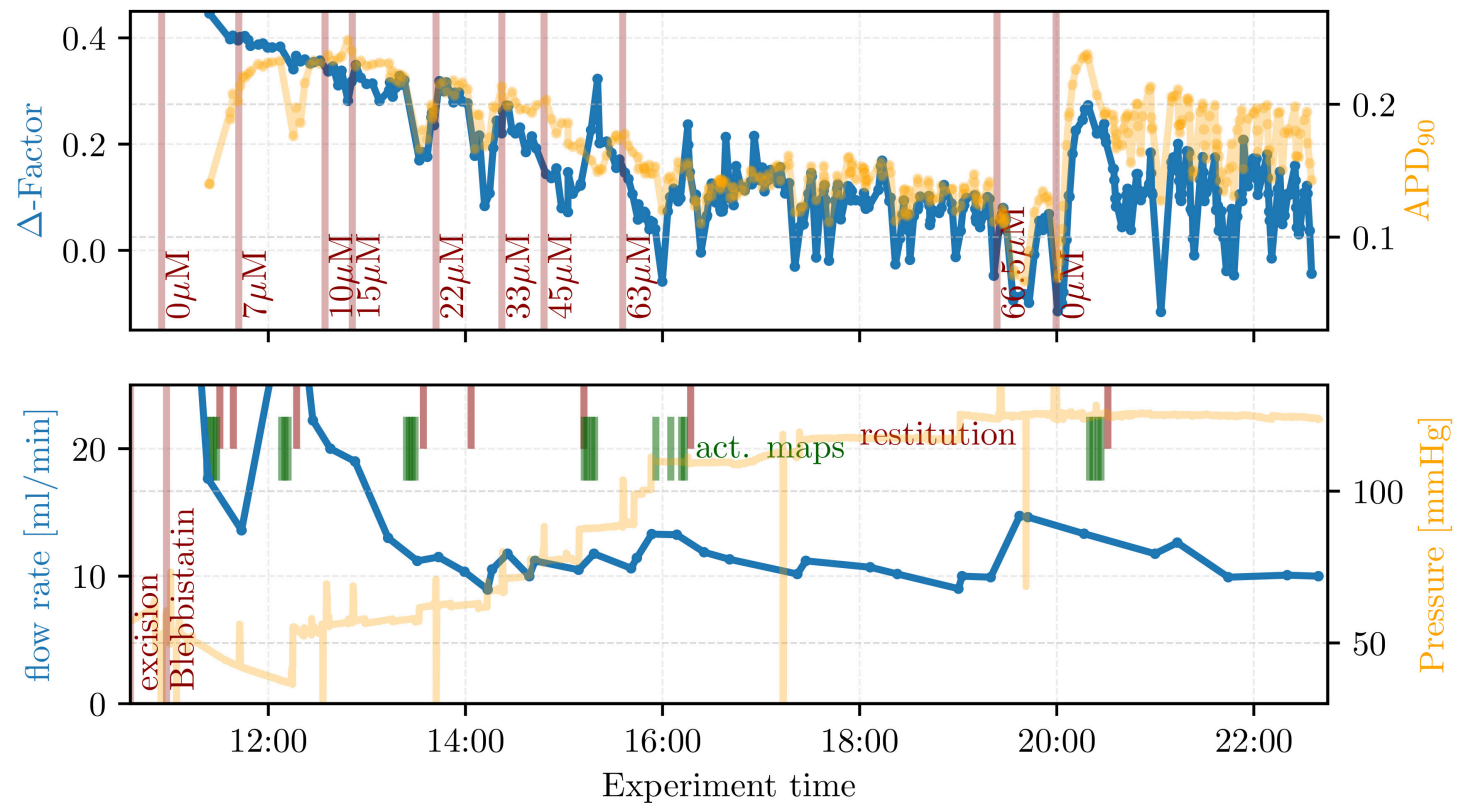

Fig. A.5 Overview plot summarizing the 2017-10-17 Experiment. The plot shows the triangularity index, APD as well as flow rate and pressure over time. Important times such as the change in Pinacidil concentration (red lines, top panel as $\mu \mathrm{M}$ ) are indicated. 

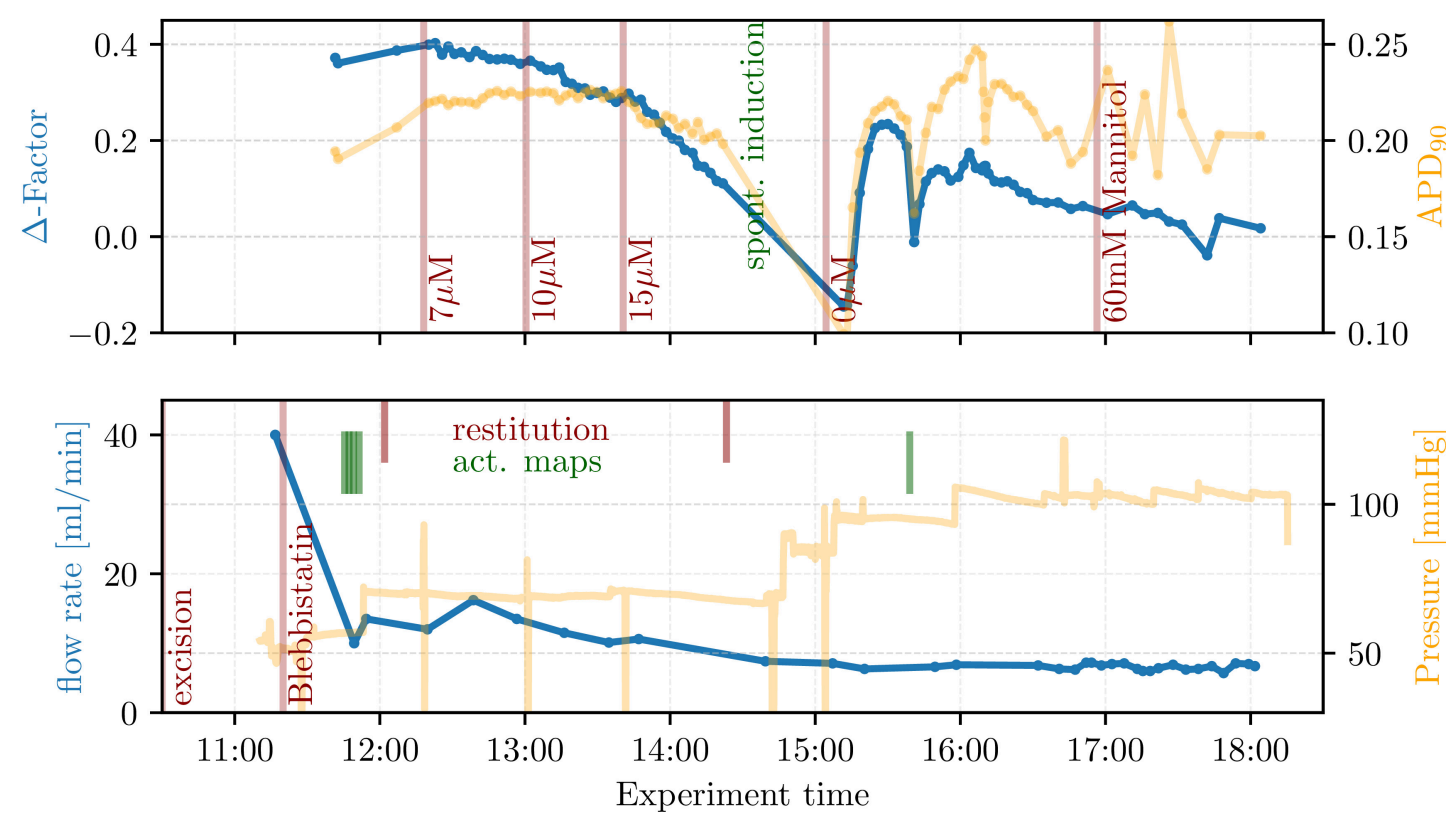

Fig. A.6 Overview plot summarizing the 2017-11-02 Experiment. The plot shows the triangularity index, APD as well as flow rate and pressure over time. Important times such as the change in Pinacidil concentration (red lines, top panel as $\mu \mathrm{M}$ ) are indicated.
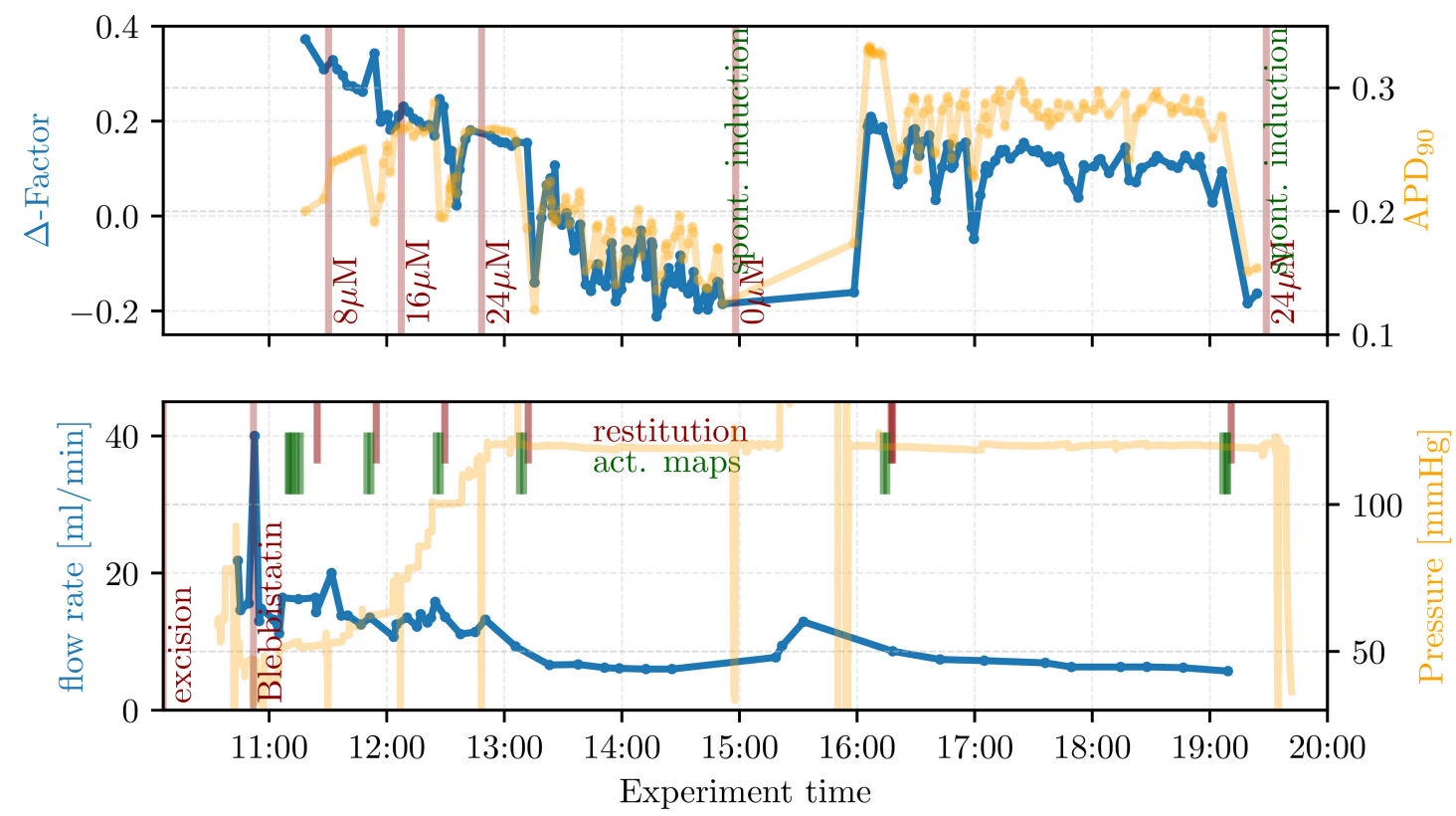

Fig. A.7 Overview plot summarizing the 2017-11-16 Experiment. The plot shows the triangularity index, APD as well as flow rate and pressure over time. Important times such as the change in Pinacidil concentration (red lines, top panel as $\mu \mathrm{M}$ ) are indicated. 

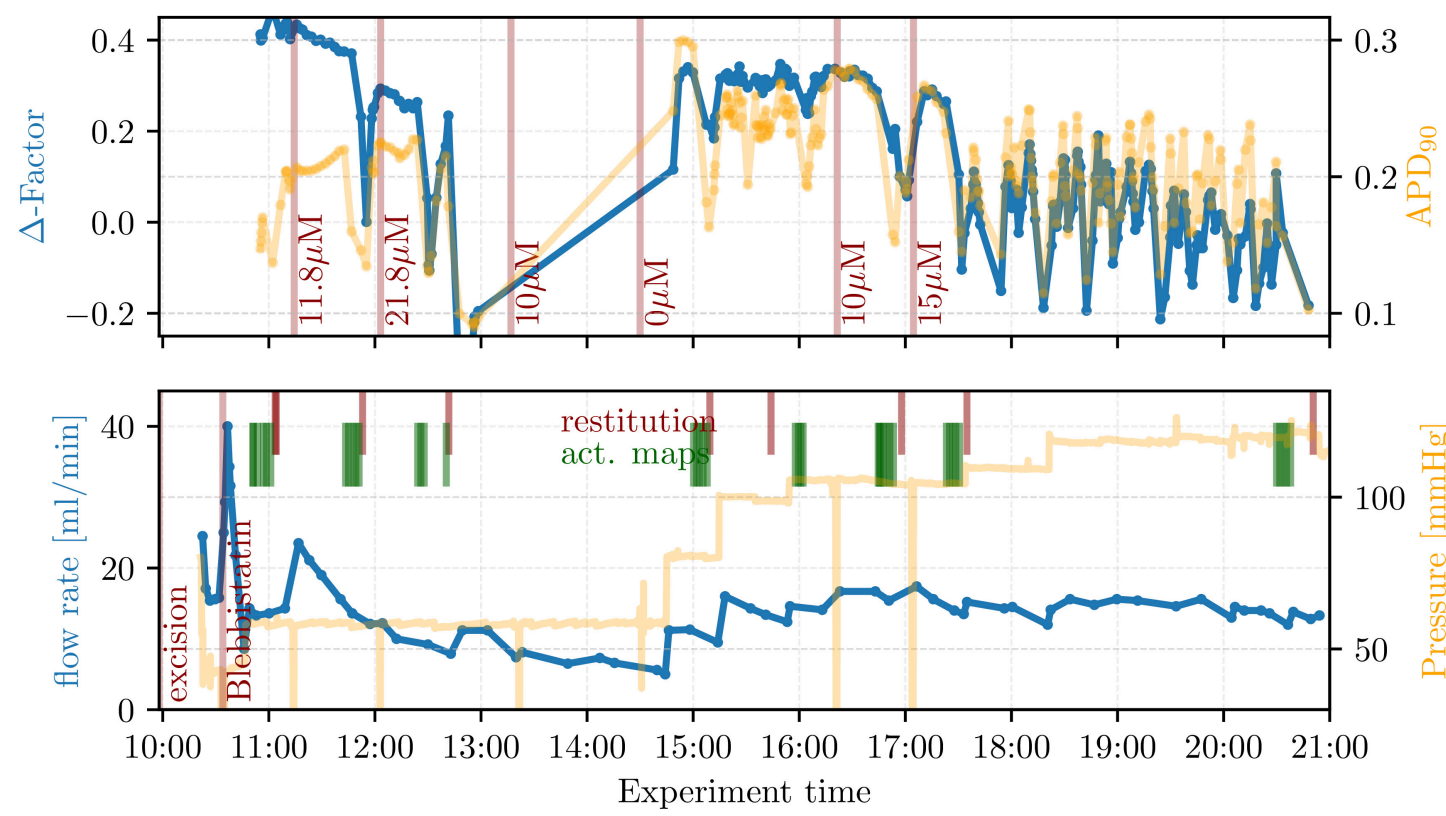

Fig. A.8 Overview plot summarizing the 2017-11-23 Experiment. The plot shows the triangularity index, APD as well as flow rate and pressure over time. Important times such as the change in Pinacidil concentration (red lines, top panel as $\mu \mathrm{M}$ ) are indicated.
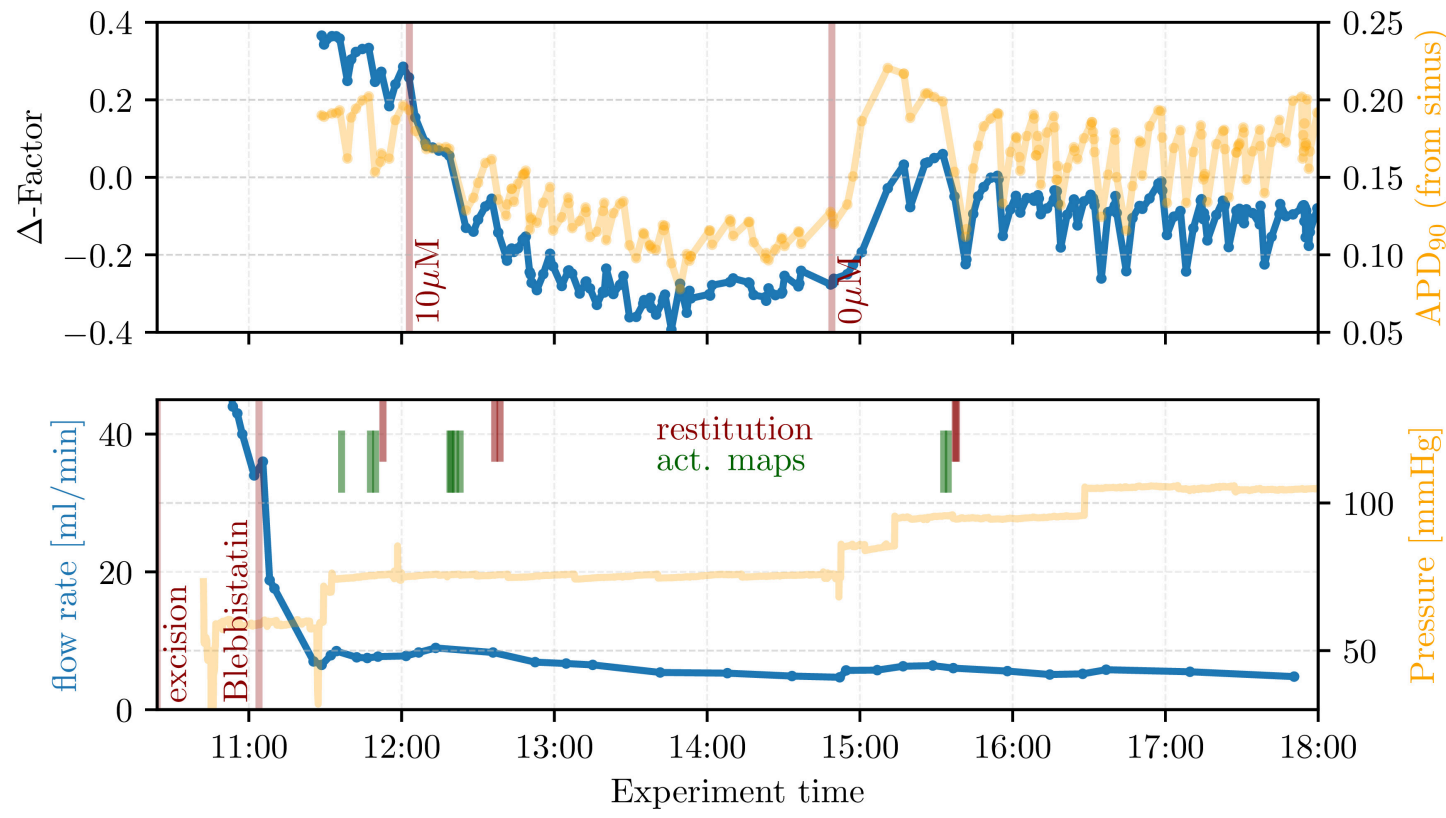

Fig. A.9 Overview plot summarizing the 2017-11-30 Experiment. The plot shows the triangularity index, APD as well as flow rate and pressure over time. Important times such as the change in Pinacidil concentration (red lines, top panel as $\mu \mathrm{M}$ ) are indicated. 

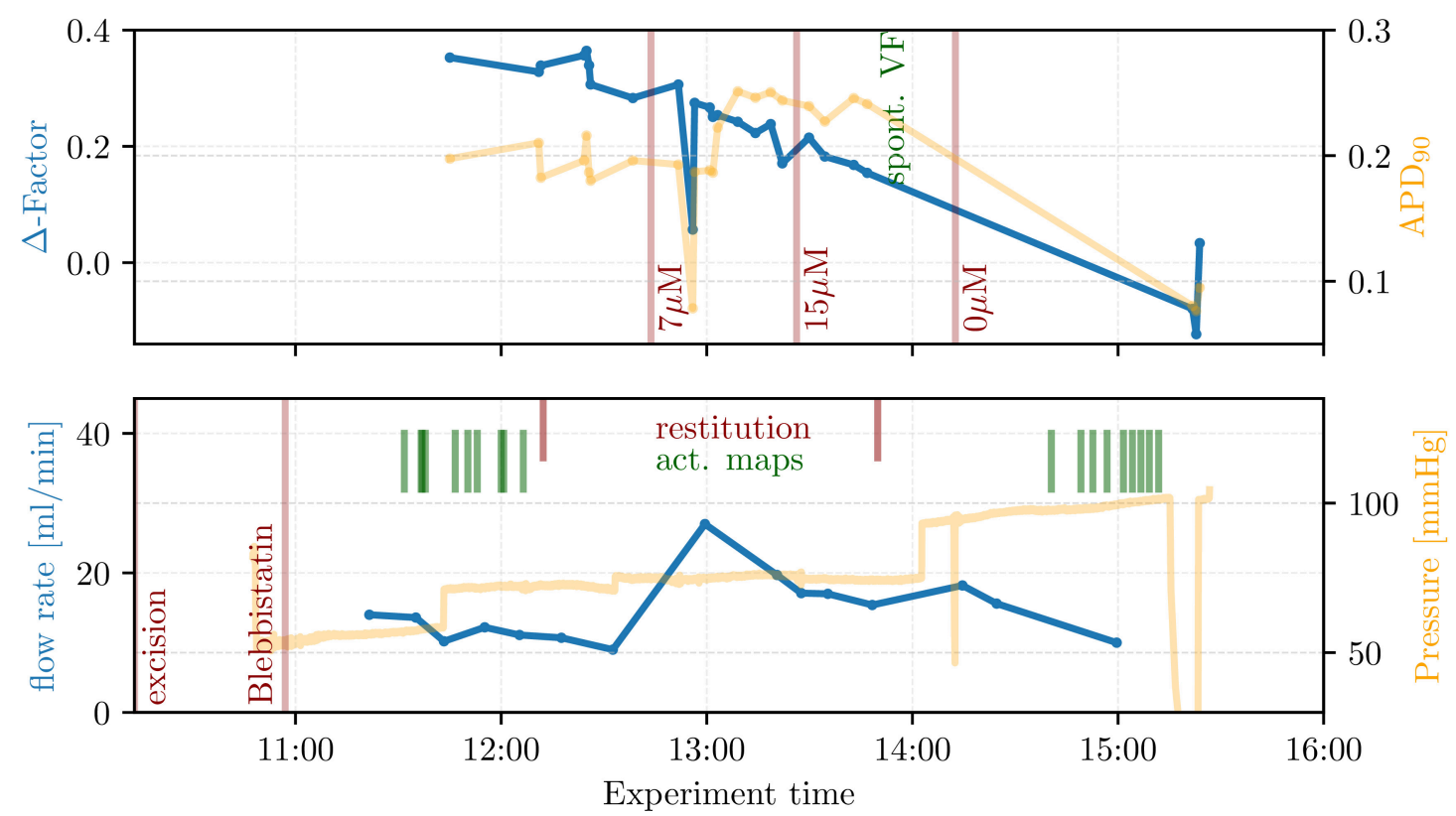

Fig. A.10 Overview plot summarizing the 2018-03-28 Experiment. The plot shows the triangularity index, APD as well as flow rate and pressure over time. Important times such as the change in Pinacidil concentration (red lines, top panel as $\mu \mathrm{M}$ ) are indicated. 


\section{A.2 Defibrillation Testing}

Although defibrillation testing is not the central topic of this thesis, one goal of the experiments was to test defibrillation in different conditions. Unfortunately, enough defibrillation events to establish the defibrillation threshold $E_{50}$, the energy required to defibrillate with a probability of $50 \%$ could not be achieved in all experiments. However, in the following, some results from the defibrillation testing that was performed in these experiments is given for context.

The defibrillation results are grouped into the time spans also used for all other analysis. These time spans differ in the Pinacidil concentration and are limited to stable conditions and optical quality. The $E_{50}$ values are estimated and given in Table A.2. Here, the energy reported is the energy for a single shock, so that the multi shock protocols require a larger total energy. The 5 shock events were performed at underdrive pacing - so slightly below the frequency of the fibrillation - and the 50 shock events performed with overdrive pacing. Shock width and other details are briefly explained in the table caption. These results are grouped by the same time spans also used in the later analysis. Since these do not include videos of poorer video quality some defibrillation events may not be included. Only time spans that contain at least two successful and two unsuccessful defibrillation attempts are listed. Further, in two cases the regression procedure did not provide a reasonable $E_{50}$ due to poor sampling. In general, values should be interpreted with care for the 50 shock values and those values calculated from few defibrillation trials. 
Table A.2 Estimated defibrillation threshold and number of successful and unsuccessful events for the different days and time spans. Each time span has a certain Pinacidil level (p.) and is split into trials with 1 shock, 5 shocks overdrive, and 50 shocks underdrive. Thus, 5 shocks are given at a higher and the 50 given at a lower frequency than the arrhythmia. The $E_{50}$ is estimated using logistic regression, with the error being estimated using a simulated experimental protocol. Because of differences in the protocol, no reasonable error can be given for the 50 shock energies. The protocol difference is also the reason for the much larger amount of unsuccessfull shocks there. The error estimation procedure is described in more detail in the $\mathrm{PhD}$ thesis of Henrik tom Wörden. The energy is calculated with an approximated impedance of $20 \Omega$. All shocks were biphasic with a rectangular form consisting of a $5 \mathrm{~ms}$ shock followed by a $2 \mathrm{~ms}$ of inverted polarity. For the 50 shocks, only monophasic $5 \mathrm{~ms}$ shocks were used. Energies refer to the energy of a single shock.

\begin{tabular}{|c|c|c|c|c|c|c|}
\hline day & time & p. $[\mu \mathrm{M}]$ & shocks & $E_{50}[\mathrm{~J}]$ & successful & unsuccessful \\
\hline \multirow[t]{2}{*}{ 2017-09-21 } & $19: 30-20: 20$ & 0 & 1 & $1.81 \pm 0.35$ & 4 & 5 \\
\hline & & & 5 & $1.62 \pm 0.31$ & 3 & 8 \\
\hline \multirow[t]{5}{*}{$2017-10-05$} & 13:00-16:00 & 7 & 1 & $1.19 \pm 0.21$ & 7 & 5 \\
\hline & & & 5 & $0.68 \pm 0.13$ & 6 & 5 \\
\hline & & & 50 & 0.41 & 8 & 39 \\
\hline & $16: 40-18: 50$ & 0 & 1 & $0.84 \pm 0.18$ & 4 & 4 \\
\hline & & & 5 & $0.53 \pm 0.12$ & 5 & 2 \\
\hline \multirow[t]{3}{*}{ 2017-10-12 } & $16: 00-18: 10$ & 0 & 1 & $0.77 \pm 0.16$ & 5 & 3 \\
\hline & & & 5 & $0.39 \pm 0.09$ & 6 & 2 \\
\hline & & & 50 & 0.78 & 5 & 34 \\
\hline \multirow[t]{4}{*}{$2017-10-17$} & $16: 00-19: 15$ & 63 & 1 & $1.72 \pm 0.27$ & 8 & 8 \\
\hline & & & 5 & $1.22 \pm 0.19$ & 8 & 8 \\
\hline & & & 50 & 1.26 & 8 & 49 \\
\hline & $20: 15-22: 10$ & 0 & 50 & 0.43 & 4 & 4 \\
\hline \multirow[t]{6}{*}{ 2017-11-16 } & $13: 10-14: 20$ & 24 & 1 & $1.48 \pm 0.35$ & 3 & 3 \\
\hline & & & 50 & 0.66 & 4 & 16 \\
\hline & $14: 20-15: 00$ & 24 & 50 & 0.77 & 2 & 12 \\
\hline & $16: 05-18: 50$ & 0 & 1 & $1.05 \pm 0.20$ & 6 & 5 \\
\hline & & & 5 & $0.80 \pm 0.15$ & 5 & 5 \\
\hline & & & 50 & 0.61 & 5 & 35 \\
\hline \multirow[t]{2}{*}{$2017-11-23$} & 18:00-19:50 & 15 & 1 & $1.40 \pm 0.23$ & 5 & 7 \\
\hline & & & 5 & $1.11 \pm 0.21$ & 3 & 8 \\
\hline \multirow[t]{6}{*}{ 2017-11-30 } & $12: 30-14: 45$ & 10 & 1 & $0.72 \pm 0.17$ & 4 & 3 \\
\hline & & & 5 & $0.30 \pm 0.07$ & 6 & 2 \\
\hline & & & 50 & 0.58 & 3 & 5 \\
\hline & $16: 00-17: 50$ & 0 & 1 & $0.54 \pm 0.11$ & 4 & 5 \\
\hline & & & 5 & $0.35 \pm 0.07$ & 3 & 8 \\
\hline & & & 50 & 0.61 & 7 & 26 \\
\hline
\end{tabular}




\section{A.3 List of all Activation Map Measurement Fits}

The following table lists the fitting parameters for all activation maps recorded in the Pinacidil series (compare Sec. 5.5). That is the slope parameter $\beta$, and the offset parameter encoded as $\tau(20 \mathrm{~V})$ and $V_{20 \mathrm{~ms}}$. Errors for $\beta$ are standard errors as estimated from the fit covariance.

Each activation map measurement is identified by the experiment day, the recording time as well as by its pulse width (p.w.) and field polarity (pol).

\begin{tabular}{|c|c|c|c|c|c|c|c|}
\hline day & time & P. $[\mu \mathrm{M}]$ & pol & p.w. $[\mathrm{ms}]$ & $\beta$ & $\tau_{10 \mathrm{~V}}$ & $\mathrm{~V}_{20 \mathrm{~ms}}$ \\
\hline \multirow[t]{32}{*}{ 2017-09-21 } & $11: 38$ & 0.0 & + & 5 & $-0.855 \pm 0.139$ & 31.8 & 17.2 \\
\hline & $11: 40$ & 0.0 & - & 5 & $-0.755 \pm 0.049$ & 25.9 & 14.1 \\
\hline & 11:41 & 0.0 & - & 2 & $-0.786 \pm 0.076$ & 31.1 & 17.6 \\
\hline & $11: 42$ & 0.0 & + & 2 & $-0.698 \pm 0.085$ & 28.5 & 16.6 \\
\hline & $13: 03$ & 1.0 & + & 2 & $-0.574 \pm 0.046$ & 30.8 & 21.2 \\
\hline & $13: 04$ & 1.0 & - & 2 & $-0.716 \pm 0.035$ & 34.7 & 21.6 \\
\hline & $13: 05$ & 1.0 & - & 5 & $-0.762 \pm 0.032$ & 31.0 & 17.7 \\
\hline & $13: 07$ & 1.0 & + & 5 & $-0.641 \pm 0.055$ & 26.3 & 15.3 \\
\hline & $13: 35$ & 2.0 & + & 5 & $-0.701 \pm 0.126$ & 33.1 & 20.5 \\
\hline & $13: 36$ & 2.0 & + & 2 & $-0.516 \pm 0.021$ & 27.2 & 18.1 \\
\hline & $13: 38$ & 2.0 & - & 2 & $-0.663 \pm 0.037$ & 33.5 & 21.7 \\
\hline & $13: 39$ & 2.0 & - & 5 & $-0.733 \pm 0.027$ & 31.5 & 18.6 \\
\hline & $14: 16$ & 3.0 & + & 5 & $-0.639 \pm 0.078$ & 31.1 & 20.0 \\
\hline & $14: 17$ & 3.0 & + & 2 & $-0.505 \pm 0.024$ & 28.0 & 19.5 \\
\hline & $14: 19$ & 3.0 & - & 2 & $-0.618 \pm 0.061$ & 32.6 & 22.1 \\
\hline & $14: 21$ & 3.0 & - & 5 & $-0.732 \pm 0.061$ & 32.0 & 19.0 \\
\hline & $14: 59$ & 5.0 & + & 5 & $-0.615 \pm 0.070$ & 32.2 & 21.7 \\
\hline & $15: 00$ & 5.0 & + & 2 & $-0.478 \pm 0.020$ & 28.8 & 21.4 \\
\hline & $15: 01$ & 5.0 & - & 2 & $-0.660 \pm 0.068$ & 34.6 & 22.9 \\
\hline & $15: 03$ & 5.0 & - & 5 & $-0.738 \pm 0.044$ & 32.1 & 19.0 \\
\hline & $15: 50$ & 10.0 & + & 5 & $-0.664 \pm 0.079$ & 31.3 & 19.6 \\
\hline & $15: 51$ & 10.0 & + & 2 & $-0.539 \pm 0.034$ & 29.6 & 20.7 \\
\hline & $15: 52$ & 10.0 & - & 2 & $-0.742 \pm 0.107$ & 37.7 & 23.5 \\
\hline & $15: 54$ & 10.0 & - & 5 & $-0.783 \pm 0.064$ & 33.0 & 19.0 \\
\hline & $18: 52$ & 0.0 & + & 5 & $-0.592 \pm 0.064$ & 32.4 & 22.6 \\
\hline & $18: 54$ & 0.0 & + & 2 & $-0.495 \pm 0.051$ & 30.8 & 23.9 \\
\hline & $18: 55$ & 0.0 & - & 2 & $-0.711 \pm 0.068$ & 40.2 & 26.6 \\
\hline & $18: 57$ & 0.0 & - & 5 & $-0.730 \pm 0.044$ & 34.5 & 21.1 \\
\hline & $20: 42$ & 15.0 & + & 5 & $-0.647 \pm 0.052$ & 30.9 & 19.6 \\
\hline & $20: 43$ & 15.0 & + & 2 & $-0.552 \pm 0.013$ & 30.4 & 21.3 \\
\hline & $20: 44$ & 15.0 & - & 2 & $-0.684 \pm 0.032$ & 34.1 & 21.8 \\
\hline & $20: 46$ & 15.0 & - & 5 & $-0.727 \pm 0.030$ & 30.3 & 17.7 \\
\hline 2017-09-28 & $11: 59$ & 0.0 & + & 5 & $-0.729 \pm 0.041$ & 20.6 & 10.4 \\
\hline
\end{tabular}




\begin{tabular}{|c|c|c|c|c|c|c|c|}
\hline day & time & P. $[\mu \mathrm{M}]$ & pol & p.w. $[\mathrm{ms}]$ & $\beta$ & $\tau_{10 \mathrm{~V}}$ & $\mathrm{~V}_{20 \mathrm{~ms}}$ \\
\hline & $12: 00$ & 0.0 & + & 2 & $-0.672 \pm 0.032$ & 20.5 & 10.4 \\
\hline & $12: 03$ & 0.0 & - & 5 & $-0.794 \pm 0.027$ & 19.5 & 9.7 \\
\hline & $12: 36$ & 10.0 & + & 5 & $-0.892 \pm 0.080$ & 21.7 & 11.0 \\
\hline & $12: 38$ & 10.0 & + & 2 & $-0.919 \pm 0.075$ & 24.9 & 12.7 \\
\hline & $12: 40$ & 10.0 & - & 2 & $-0.875 \pm 0.077$ & 20.8 & 10.5 \\
\hline & $13: 48$ & 0.0 & + & 5 & $-0.691 \pm 0.032$ & 18.9 & 9.21 \\
\hline & $13: 50$ & 0.0 & + & 2 & $-0.665 \pm 0.026$ & 20.3 & 10.2 \\
\hline & $13: 52$ & 0.0 & - & 2 & $-0.693 \pm 0.020$ & 19.1 & 9.36 \\
\hline & $13: 54$ & 0.0 & - & 5 & $-0.686 \pm 0.011$ & 16.8 & 7.73 \\
\hline & $14: 16$ & 0.0 & + & 5 & $-0.637 \pm 0.014$ & 18.2 & 8.61 \\
\hline & $14: 19$ & 0.0 & + & 2 & $-0.626 \pm 0.026$ & 19.5 & 9.63 \\
\hline & $14: 20$ & 0.0 & - & 2 & $-0.645 \pm 0.051$ & 18.8 & 9.08 \\
\hline & $14: 33$ & 0.0 & - & 5 & $-0.656 \pm 0.024$ & 16.9 & 7.76 \\
\hline & $15: 46$ & 7.0 & + & 5 & $-0.661 \pm 0.032$ & 17.1 & 7.9 \\
\hline & $15: 48$ & 7.0 & + & 2 & $-0.701 \pm 0.011$ & 19.2 & 9.42 \\
\hline \multirow{16}{*}{ 2017-10-05 } & $12: 19$ & 0.0 & + & 5 & $-0.865 \pm 0.047$ & 18.0 & 8.87 \\
\hline & $12: 21$ & 0.0 & + & 2 & $-0.897 \pm 0.091$ & 19.3 & 9.62 \\
\hline & $12: 24$ & 0.0 & - & 2 & $-0.930 \pm 0.044$ & 20.7 & 10.4 \\
\hline & $12: 26$ & 0.0 & - & 5 & $-0.872 \pm 0.044$ & 18.0 & 8.84 \\
\hline & $13: 01$ & 7.0 & + & 5 & $-0.891 \pm 0.049$ & 16.6 & 8.1 \\
\hline & $13: 03$ & 7.0 & + & 2 & $-1.057 \pm 0.060$ & 19.7 & 9.87 \\
\hline & $13: 05$ & 7.0 & - & 2 & $-1.122 \pm 0.110$ & 21.0 & 10.4 \\
\hline & $13: 07$ & 7.0 & - & 5 & $-1.044 \pm 0.061$ & 18.7 & 9.37 \\
\hline & $15: 53$ & 7.0 & + & 5 & $-0.947 \pm 0.044$ & 19.1 & 9.53 \\
\hline & $15: 54$ & 7.0 & + & 2 & $-1.039 \pm 0.064$ & 23.7 & 11.8 \\
\hline & $15: 57$ & 7.0 & - & 2 & $-1.069 \pm 0.082$ & 23.5 & 11.6 \\
\hline & $15: 59$ & 7.0 & - & 5 & $-1.077 \pm 0.056$ & 21.4 & 10.6 \\
\hline & $16: 45$ & 0.0 & + & 5 & $-0.842 \pm 0.047$ & 22.2 & 11.3 \\
\hline & $16: 47$ & 0.0 & + & 2 & $-0.874 \pm 0.048$ & 23.7 & 12.1 \\
\hline & $16: 49$ & 0.0 & - & 2 & $-0.966 \pm 0.071$ & 24.2 & 12.2 \\
\hline & $16: 51$ & 0.0 & - & 5 & $-0.943 \pm 0.053$ & 20.5 & 10.3 \\
\hline \multirow[t]{8}{*}{ 2017-10-12 } & $12: 31$ & 0.0 & + & 5 & $-0.794 \pm 0.053$ & 22.0 & 11.3 \\
\hline & $12: 33$ & 0.0 & + & 2 & $-0.802 \pm 0.033$ & 24.7 & 13.0 \\
\hline & $12: 35$ & 0.0 & - & 2 & $-0.732 \pm 0.059$ & 25.1 & 13.6 \\
\hline & $12: 37$ & 0.0 & - & 5 & $-0.747 \pm 0.050$ & 21.8 & 11.2 \\
\hline & $15: 45$ & 0.0 & + & 5 & $-0.679 \pm 0.008$ & 18.8 & 9.14 \\
\hline & $18: 48$ & 5.0 & + & 5 & $-0.680 \pm 0.034$ & 19.7 & 9.77 \\
\hline & $18: 53$ & 5.0 & - & 5 & $-0.844 \pm 0.050$ & 20.9 & 10.6 \\
\hline & $18: 55$ & 5.0 & - & 2 & $-0.725 \pm 0.050$ & 21.2 & 10.8 \\
\hline \multirow[t]{3}{*}{ 2017-10-17 } & $11: 22$ & 0.0 & + & 5 & $-0.861 \pm 0.021$ & 26.3 & 13.8 \\
\hline & $11: 24$ & 0.0 & + & 2 & $-0.847 \pm 0.039$ & 28.4 & 15.1 \\
\hline & $11: 26$ & 0.0 & - & 2 & $-0.973 \pm 0.074$ & 31.5 & 15.9 \\
\hline
\end{tabular}




\begin{tabular}{|c|c|c|c|c|c|c|c|}
\hline day & time & $\mathrm{P} .[\mu \mathrm{M}]$ & pol & p.w. $[\mathrm{ms}]$ & $\beta$ & $\tau_{10 \mathrm{~V}}$ & $\mathrm{~V}_{20 \mathrm{~ms}}$ \\
\hline & $11: 28$ & 0.0 & - & 5 & $-0.964 \pm 0.041$ & 26.4 & 13.3 \\
\hline & $12: 08$ & 7.0 & + & 5 & $-0.818 \pm 0.112$ & 25.8 & 13.6 \\
\hline & $12: 10$ & 7.0 & + & 2 & $-0.795 \pm 0.049$ & 27.5 & 14.9 \\
\hline & $12: 12$ & 7.0 & - & 2 & $-0.887 \pm 0.079$ & 29.4 & 15.4 \\
\hline & $13: 27$ & 15.0 & - & 2 & $-0.884 \pm 0.161$ & 31.5 & 16.7 \\
\hline & $13: 29$ & 15.0 & - & 5 & $-0.842 \pm 0.099$ & 24.6 & 12.8 \\
\hline & $15: 12$ & 45.0 & + & 5 & $-0.731 \pm 0.075$ & 25.9 & 14.3 \\
\hline & $15: 18$ & 45.0 & - & 5 & $-0.865 \pm 0.092$ & 27.6 & 14.5 \\
\hline & $16: 11$ & 63.0 & - & 2 & $-0.782 \pm 0.101$ & 33.6 & 19.4 \\
\hline & $16: 13$ & 63.0 & - & 5 & $-0.803 \pm 0.128$ & 29.7 & 16.4 \\
\hline & $20: 20$ & 0.0 & + & 5 & $-0.609 \pm 0.036$ & 26.4 & 15.8 \\
\hline & $20: 22$ & 0.0 & + & 2 & $-0.547 \pm 0.034$ & 26.3 & 16.4 \\
\hline & $20: 25$ & 0.0 & - & 2 & $-0.748 \pm 0.149$ & 30.7 & 17.7 \\
\hline & $20: 26$ & 0.0 & - & 5 & $-0.733 \pm 0.068$ & 25.4 & 13.9 \\
\hline \multirow[t]{4}{*}{ 2017-11-02 } & $11: 45$ & 0.0 & + & 5 & $-0.815 \pm 0.030$ & 22.2 & 11.3 \\
\hline & $11: 47$ & 0.0 & - & 5 & $-0.788 \pm 0.052$ & 22.2 & 11.4 \\
\hline & $11: 49$ & 0.0 & - & 2 & $-0.800 \pm 0.075$ & 25.1 & 13.3 \\
\hline & $11: 51$ & 0.0 & + & 2 & $-0.791 \pm 0.051$ & 22.5 & 11.6 \\
\hline \multirow[t]{14}{*}{ 2017-11-16 } & $11: 10$ & 0.0 & + & 5 & $-0.810 \pm 0.044$ & 31.5 & 17.5 \\
\hline & $11: 12$ & 0.0 & - & 5 & $-0.827 \pm 0.070$ & 29.7 & 16.1 \\
\hline & $11: 13$ & 0.0 & - & 2 & $-0.873 \pm 0.056$ & 31.2 & 16.7 \\
\hline & $11: 15$ & 0.0 & + & 2 & $-0.763 \pm 0.024$ & 27.8 & 15.4 \\
\hline & $11: 49$ & 8.0 & + & 5 & $-0.828 \pm 0.095$ & 32.7 & 18.1 \\
\hline & $11: 51$ & 8.0 & - & 5 & $-0.735 \pm 0.035$ & 27.4 & 15.4 \\
\hline & $12: 25$ & 16.0 & - & 5 & $-0.788 \pm 0.040$ & 33.0 & 18.9 \\
\hline & $12: 27$ & 16.0 & + & 5 & $-0.738 \pm 0.059$ & 29.2 & 16.7 \\
\hline & $13: 07$ & 24.0 & + & 5 & $-0.861 \pm 0.106$ & 37.8 & 20.9 \\
\hline & $13: 09$ & 24.0 & - & 5 & $-0.822 \pm 0.078$ & 33.4 & 18.6 \\
\hline & $16: 13$ & 0.0 & - & 5 & $-0.738 \pm 0.049$ & 34.3 & 20.7 \\
\hline & $16: 15$ & 0.0 & + & 5 & $-0.684 \pm 0.021$ & 29.8 & 17.9 \\
\hline & 19:06 & 0.0 & + & 5 & $-0.741 \pm 0.027$ & 34.6 & 21.0 \\
\hline & 19:09 & 0.0 & - & 5 & $-0.798 \pm 0.035$ & 37.0 & 21.6 \\
\hline \multirow[t]{10}{*}{$2017-11-23$} & $10: 51$ & 0.0 & + & 5 & $-0.863 \pm 0.030$ & 37.1 & 20.4 \\
\hline & $10: 53$ & 0.0 & - & 5 & $-0.822 \pm 0.040$ & 32.6 & 18.2 \\
\hline & $10: 56$ & 0.0 & - & 2 & $-0.845 \pm 0.084$ & 35.3 & 19.6 \\
\hline & $10: 58$ & 0.0 & + & 2 & $-0.853 \pm 0.072$ & 36.2 & 20.1 \\
\hline & $11: 43$ & 11.8 & + & 5 & $-0.844 \pm 0.035$ & 37.4 & 21.0 \\
\hline & $11: 45$ & 11.8 & - & 5 & $-0.787 \pm 0.058$ & 33.1 & 19.0 \\
\hline & $11: 47$ & 11.8 & - & 2 & $-0.846 \pm 0.073$ & 34.9 & 19.3 \\
\hline & $11: 49$ & 11.8 & + & 2 & $-0.846 \pm 0.073$ & 34.9 & 19.3 \\
\hline & $12: 24$ & 21.8 & + & 5 & $-0.845 \pm 0.052$ & 38.4 & 21.7 \\
\hline & $12: 26$ & 21.8 & - & 5 & $-0.797 \pm 0.059$ & 33.3 & 18.9 \\
\hline
\end{tabular}




\begin{tabular}{lllllcll}
\hline \multirow{2}{*}{ day } & & & & & $\beta$ & $\tau_{10 \mathrm{~V}}$ & $\mathrm{~V}_{20 \mathrm{~ms}}$ \\
& time & $\mathrm{P} .[\mu \mathrm{M}]$ & pol & p.w. $[\mathrm{ms}]$ & & & \\
\hline & $12: 27$ & 21.8 & + & 2 & $-0.917 \pm 0.041$ & 37.3 & 19.7 \\
$15: 00$ & 0.0 & + & 5 & $-0.934 \pm 0.098$ & 42.5 & 22.4 \\
$15: 01$ & 0.0 & - & 5 & $-0.796 \pm 0.094$ & 35.2 & 20.4 \\
$15: 03$ & 0.0 & - & 2 & $-0.798 \pm 0.181$ & 39.0 & 23.1 \\
$15: 05$ & 0.0 & + & 2 & $-0.855 \pm 0.106$ & 38.7 & 21.7 \\
$15: 57$ & 0.0 & - & 5 & $-0.963 \pm 0.159$ & 51.0 & 26.4 \\
$16: 45$ & 10.0 & + & 5 & $-0.758 \pm 0.079$ & 39.9 & 24.9 \\
$16: 47$ & 10.0 & - & 5 & $-0.641 \pm 0.083$ & 32.8 & 21.6 \\
$16: 49$ & 10.0 & - & 2 & $-0.594 \pm 0.090$ & 31.8 & 21.8 \\
$17: 23$ & 15.0 & + & 5 & $-0.731 \pm 0.086$ & 40.1 & 25.9 \\
$17: 24$ & 15.0 & - & 5 & $-0.620 \pm 0.073$ & 33.7 & 23.2 \\
$17: 26$ & 15.0 & - & 2 & $-0.562 \pm 0.069$ & 31.3 & 22.2 \\
$17: 28$ & 15.0 & + & 2 & $-0.716 \pm 0.054$ & 37.2 & 23.7 \\
$20: 29$ & 15.0 & + & 5 & $-0.750 \pm 0.059$ & 38.0 & 23.5 \\
$20: 31$ & 15.0 & - & 5 & $-0.900 \pm 0.122$ & 43.1 & 23.5 \\
$20: 33$ & 15.0 & - & 2 & $-0.705 \pm 0.102$ & 34.7 & 21.8 \\
$20: 35$ & 15.0 & + & 2 & $-0.657 \pm 0.064$ & 33.9 & 22.3 \\
$11: 47$ & 0.0 & + & 5 & $-0.834 \pm 0.033$ & 22.1 & 11.3 \\
$11: 49$ & 0.0 & - & 5 & $-0.877 \pm 0.067$ & 20.4 & 10.2 \\
$12: 19$ & 10.0 & + & 2 & $-0.867 \pm 0.057$ & 24.2 & 12.5 \\
$12: 21$ & 10.0 & + & 5 & $-0.802 \pm 0.025$ & 20.5 & 10.3 \\
$12: 22$ & 10.0 & - & 5 & $-0.873 \pm 0.157$ & 20.3 & 10.2 \\
$15: 32$ & 0.0 & + & 5 & $-0.829 \pm 0.052$ & 23.5 & 12.1 \\
$15: 34$ & 0.0 & - & 5 & $-0.860 \pm 0.064$ & 21.8 & 11.0 \\
\hline
\end{tabular}




\section{A.4 Comparison of Cycle Length Calculations}

The CL was calculated generally based on the dominant CL as described in Section 4.3.2. However, in all defibrillation events, which includes all of the $3 \mathrm{~s}$ or $5 \mathrm{~s}$ VF episodes analyzed for the Pinacidil series we have also calculated the period from the ECG. As mentioned before, it is also possible to use an averaged CL instead of the dominant one. Figure A.11 compares all three of these values. The dominant CL directly correlates with the period measured from the ECG. However, there is some differences, mostly a longer CL, when comparing the spatially averaged calculation with the dominant CL one. Generally, the ECG comparison has a higher variance. This may be due to finding the double/half period in the ECG and also due to the shorter analyzed time segment of only $1 \mathrm{~s}$. Overall, however, the agreement of the ECG and the dominant CL seems to have no or little systematic differences. 
a)

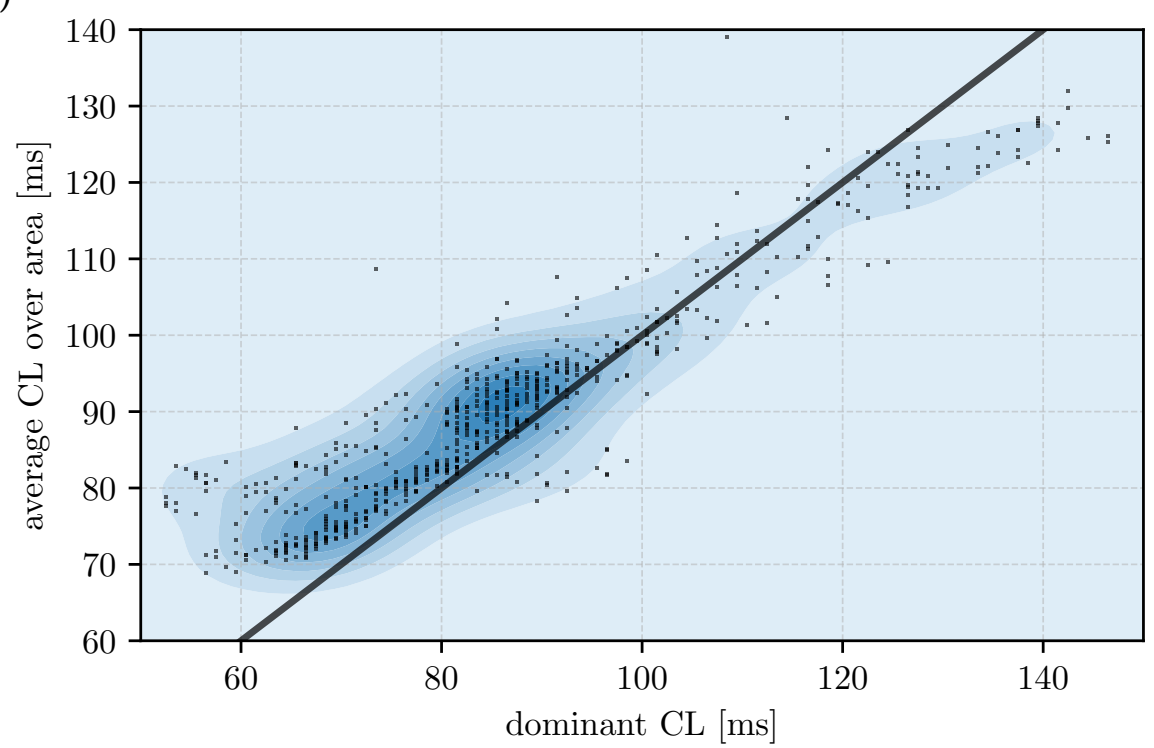

b)

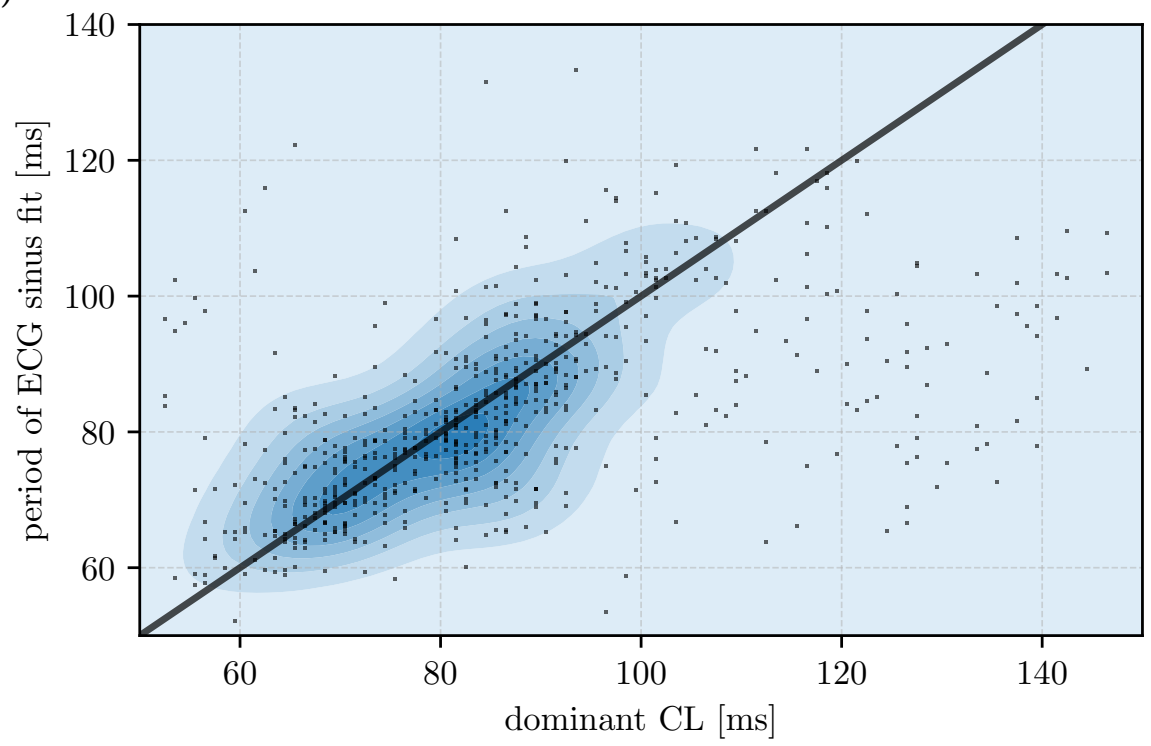

Fig. A.11 a) Kernel densitity estimation and individual episodes comparing dominant CL with the average of the CL map as introduced in Section 4.3.2. A slight shift in detected period is apparent. b) The same comparison but comparing the dominant CL with the period derived from the ECG by fitting a sine to $1 \mathrm{~s}$ of ECG time series. Aside stronger variance, these values correlate well. Overall over 600 episodes are included in the plot. 


\section{Contributions to the Annihilation Rate}

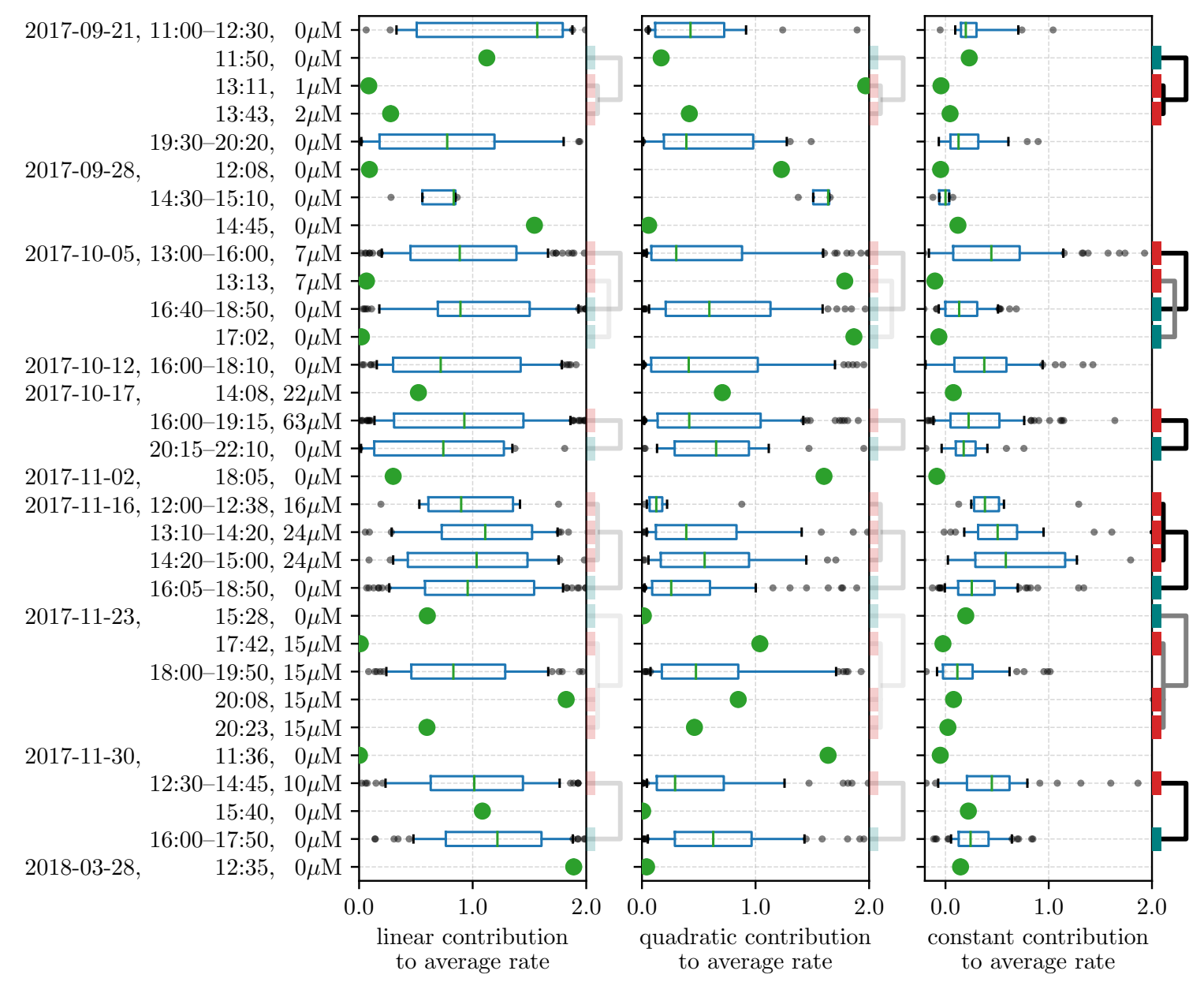

Fig. A.12 Comparison of the importance of the linear and quadratic contribution to the annihilation rate (compare Sec. 6.1.2 for discussion) for all time spans with points indicating single long videos (15 s to $30 \mathrm{~s}$ ) and boxplots for multiple short $(3 \mathrm{~s}$ or $5 \mathrm{~s}$ ) VF episodes within the time span. The $y$-axis shows the experiment day, time span, and Pinacidil concentration. The first two panels shows the average contribution of the fit parameter for the linear and quadratic term in a model without a constant offset. For comparison and to show that a constant component is not important, the third panel shows the contribution of the constant offset when an additional constant term is permitted. Brackets next to the axes (light for the first two) indicate which times can be used to compare a single heart with and without Pinacidil. Brighter/grey ones groupe single long videos. Red colour indicates Pinacidil while teal indicates no Pinacidil. 


\section{A.5 Alternative analysis of the Spatial Organiza- tion}

Here the alternative analysis of the spatial organization similar to the one performed in Section 7.2.1 is shown. The Analysis is here varied in the use of the APD and the transform:

- Figure A.13: the same analysis based on $-\Delta_{80}$ without the additional transform. (Additional smoothing is applied.)

- Figure A.13: the same analysis, but based on the $\mathrm{APD}_{80}$.

- Figure A.15: the same analysis based on the $\mathrm{APD}_{80}$ and without the additional transform. (Additional smoothing is applied.)

a)

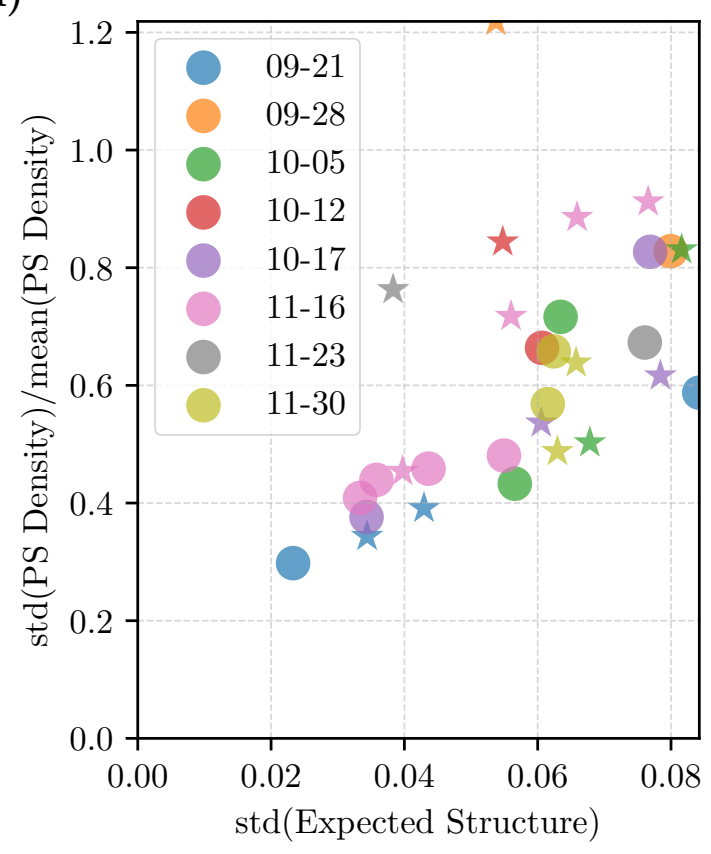

b)

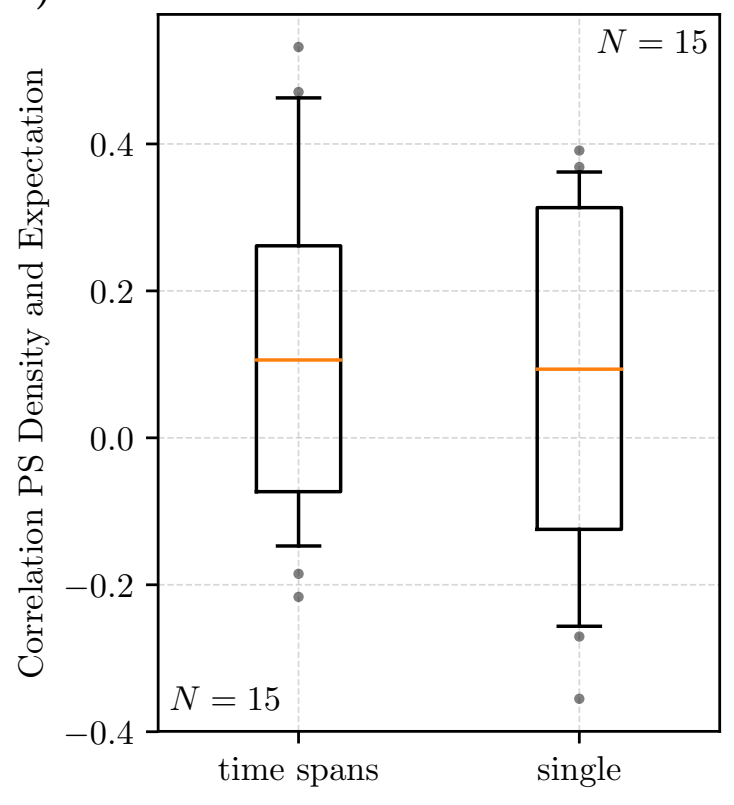

Fig. A.13 The same as Figure 7.4 but based on $-\Delta_{80}$ after additional spatial smoothing with a Gaussian function with $\sigma=5$ for similarity. a) The strength of the structuring seen in the PS density plotted against the expected structuring strength. Circles indicate time spans with many VF episodes while stars indicate a single long VF episode. Colours indicate the experiment day, and the legend also applies to the stars. b) The spatial correlation coefficient between the PS density and the expected structure. The two boxplots show the result of the time spans containing multiple VF episodes and the single long VF episodes. 
a)

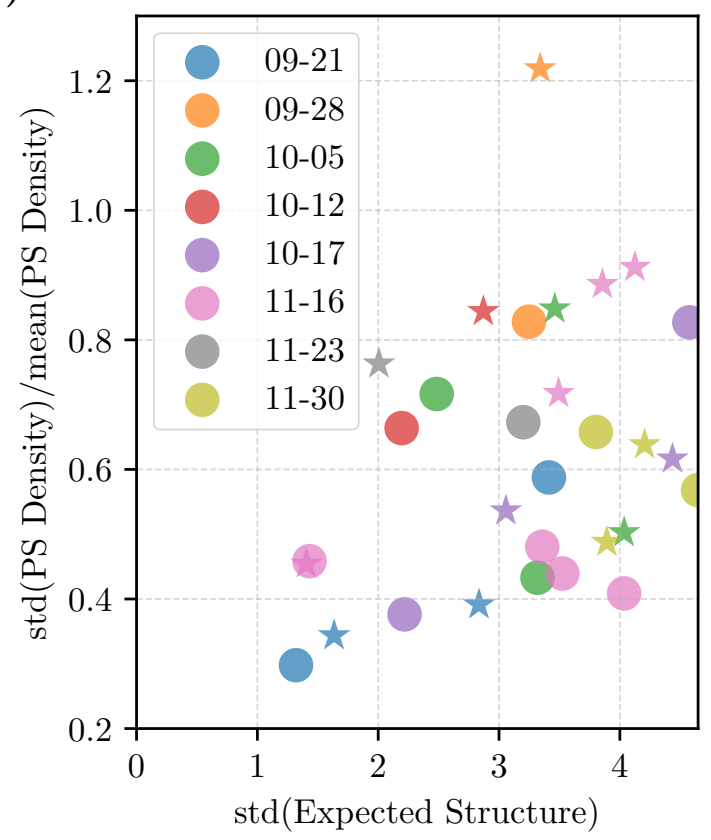

b)

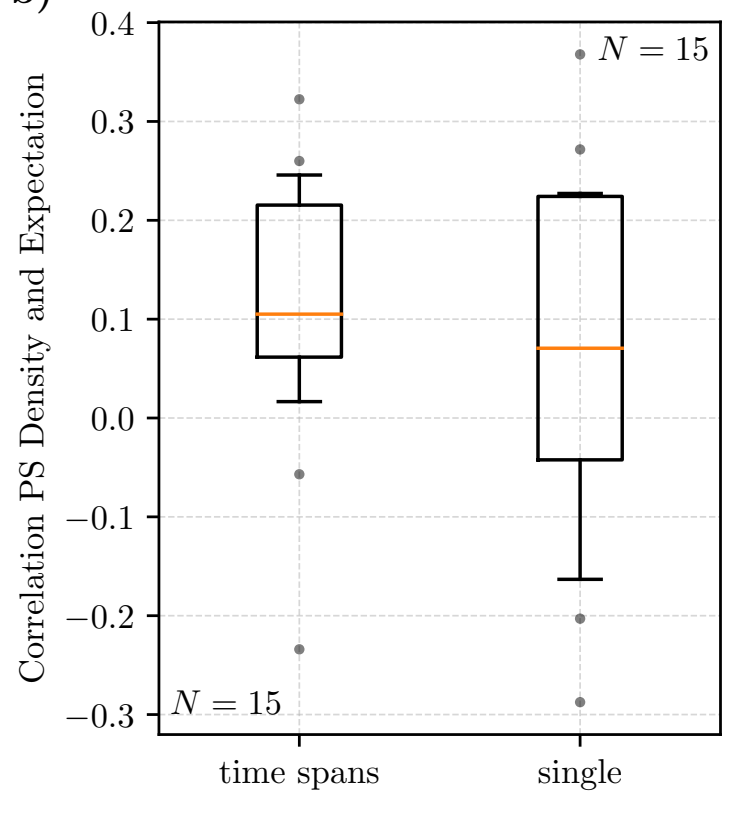

Fig. A.14 The same as Figure 7.4 but based on $\mathrm{APD}_{80}$. a) The strength of the structuring seen in the PS density plotted against the expected structuring strength. Circles indicate time spans with many VF episodes while stars indicate a single long VF episode. Colours indicate the experiment day, and the legend also applies to the stars. b) The spatial correlation coefficient between the PS density and the expected structure. The two boxplots show the result of the time spans containing multiple VF episodes and the single long VF episodes. 
a)

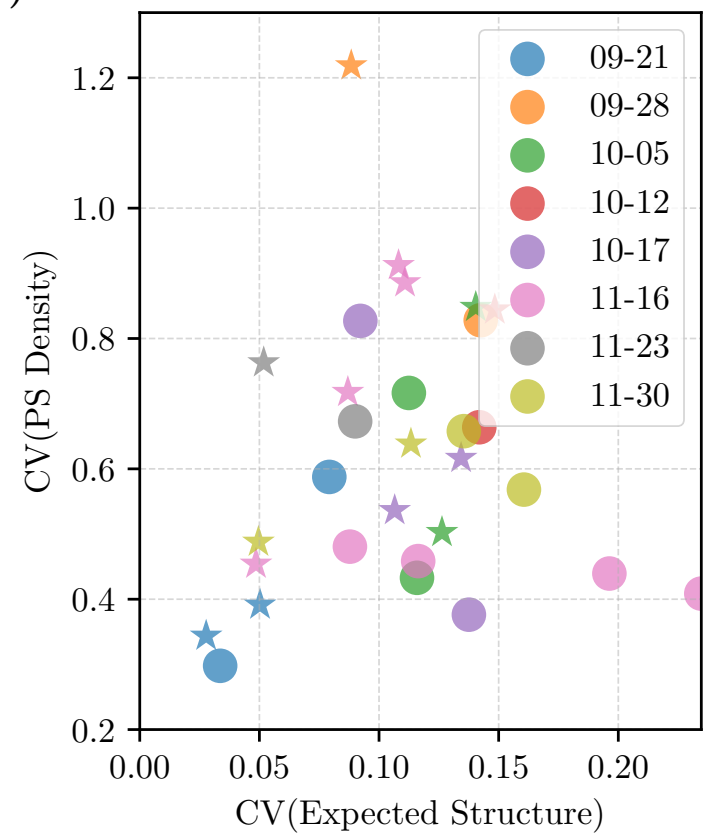

b)

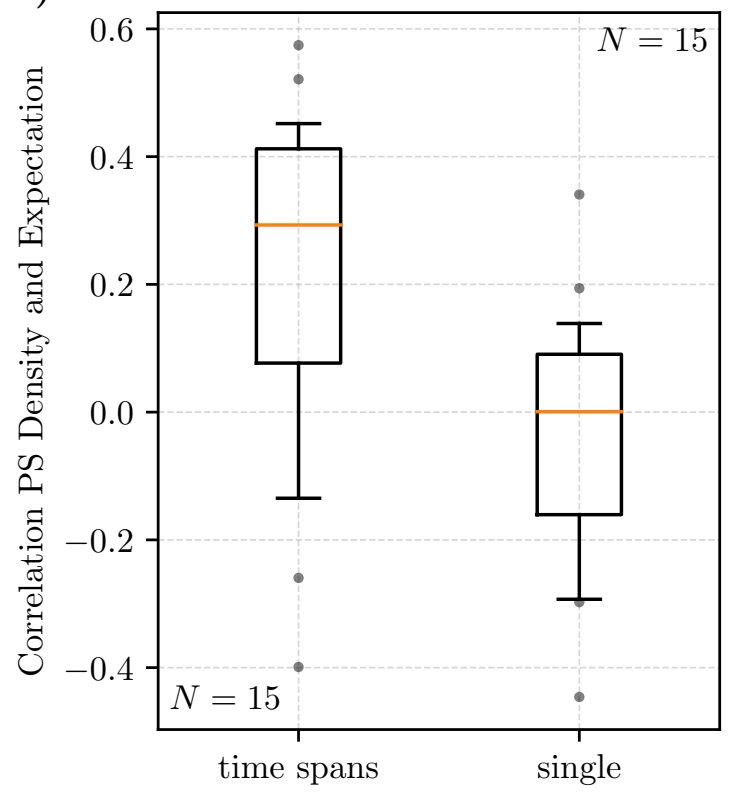

Fig. A.15 The same as Figure 7.4 but based on $-\mathrm{APD}_{80}$ after additional spatial smoothing with a Gaussian function with $\sigma=5$ for similarity. a) The strength of the structuring seen in the PS density plotted against the expected structuring strength. Circles indicate time spans with many VF episodes while stars indicate a single long VF episode. Colours indicate the experiment day, and the legend also applies to the stars. b) The spatial correlation coefficient between the PS density and the expected structure. The two boxplots show the result of the time spans containing multiple VF episodes and the single long VF episodes. 

Appendix B

Code Sample 


\section{B.1 Windowing Function for Upstroke Detection}

The below python snippet defines the exact kernel used to find the excitation times (upstrokes). The default parameters are the ones used for the analysis of arrhythmia, more details can be found in Section 4.2.

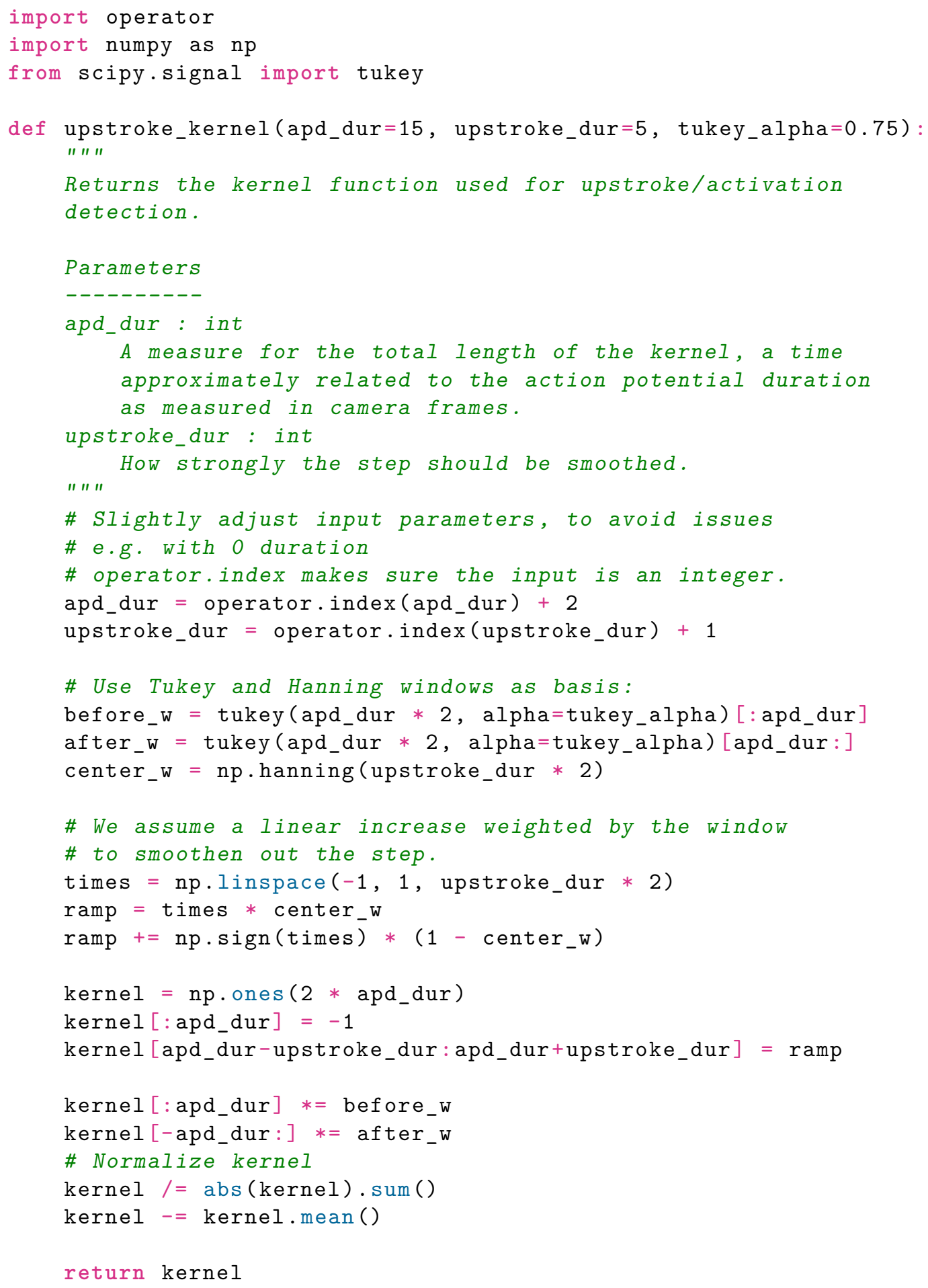




\section{Appendix C}

\section{Additional Information or Details}

\section{C.1 Experimental Recommendations}

Experimental work can become tricky and sometimes based on experience we find a certain way of doing things is successful. Some of these tips may thus not hold up to thorough scientific analysis. However, such tips may be helpful to scientists new in the fields. Thus, in this appending I provide a list of experiences gathered or taught to me when conducting experiments.

- Especially when using drugs it can sometimes happen that even the strongest shock protocols cannot defibrillate a heart or the arrhythmia self induces again very quickly. Especially if the second observation is the case a good option to try is many shocks with a fast sinus period and an amplitude such that a single shock should be able to defibrillate. In some cases this will work to defibrillate the arrhythmia.

- Pacing protocols can sometimes induce arrhythmia accidentally, at least unless timed carefully. While not always an option, especially if this occurs and is unwanted, care should be taken to reduce the amplitude as much as possible. This will make the vulnerable window smaller and can solve or at least limit the issue. An other solution should of course be to synchronize the pacing (or first shock) to the natural heart rhythm.

- Inducing arrhythmia is often conveniently done using $50 \mathrm{~Hz}$ or $60 \mathrm{~Hz}$ local or far field pacing protocols. These typically reliably induce fibrillation. As taught to me, one thing to keep an eye on is again the camera traces. If the camera traces start to look irregular there is a very high chance of arrhythmia induction. If this does not work, increasing the pacing duration or simply repeating the procedure is a good idea. If however the traces are not irregular, increasing the pacing strength or repositioning the electrode may help. Additional one can try to lower the pacing frequency since $50 \mathrm{~Hz}$ is much higher than any arrhythmia and the overall pacing strength at this high frequency might require larger energies. 
While lowering the frequency too much may fail to induce arrhythmia as well, trying out a few lower frequencies can be helpful.

- It is a good habit for the experimenter to keep an eye on the camera traces of the heart during the experiment. This allows to observe possible changes already during the experiment. This trace observations will also show if there is still movement in the heart, although the same can be achieved using a bandpass filter as implemented into our experimental software. For example, sometimes resonance with the pump can cause small movements and once found these movements can be easily avoided by increasing the pumping speed.

- Additionally observing a single pixel or small trace will provide the experimenter with an idea of whether or not the data is good enough for the purpose of analysis. And whether restaining should be considered. A single pixel trace should show a very clear action potential during sinus: with good quality, the single pixel noise amplitude will be well below the sinus rhythm amplitude. If this is not the case, even with strong filtering the data will be very hard or impossible to analyze in depth during fibrillation.

- The perfusion is sometimes better at the start of the experiment. Likely because of this, staining the heart very early on in the experiment sometimes seemed to provide for a better image quality. 


\section{C.2 Overview over PS Identification Methods}

Many methods and especially data preprocessing approaches have been described in literature and the spatial and temporal filtering can vary widely. Here many, though certainly not all, methods for phase singularity identification both for numerical and experimental data are listed. This includes a suggestion for PS identification in simulation data based on the derivatives.

The method developed by Rogers [124] and the one used in this thesis focus on improving tracking techniques by making use of the wavefront information. Most methods vary mainly in the way the phase is defined. The PS are then found in a similar manner through a line integral around the central points. However, variations exists, for example by changing the line integral length or using a combination of multiple line lengths [125]. Another approach is to find the intersection point of two iso-values. The following lists the various methods:

- Finding the points where voltage iso-value line and the line of zero derivative in the voltage intersect (only used in simulations) [126]

- Variation, I found useful: Using the local derivative not including the diffusion part for comparison provides a different quantification of the local phase. This improved PS identification noticeably in certain parameters ranges of the Fenton-Karma model.

- Using time delay embedding and the line integral. [127]

- Using the Hilbert transform and then a line integral over the phase map. Since the Hilbert transform requires a stationary signal, two variations are used:

- Subtracting the mean (after or through highpass filtering)

- Using a pseudo-empirical mode decomposition (PEMD) method. ${ }^{1}$ This decomposition follows local minima and maxima in the signal that occur on a certain time scale and shifts the signals to be stationary with respect to the local minima/maxima. Thus the signal is not required to be stationary and for example smaller amplitude events may be picked up easier. [129]

- A variation of the embedding method, however Rogers combines this with wavefront tracking to achieve a higher reliability and better tracking. [124]

- More recently suggestions were made to find areas of high phase variance [130] or, in a simulation study, to only look for points with ill defined phase locally [131]. ${ }^{2}$

- For simulated data of two variable models Davidsen et al. have used lines of zero derivative in both variables [74].

\footnotetext{
${ }^{1}$ PEMD methods are more commonly used in other fields to extract phases from non-stationary data [128].

${ }^{2}$ Neither methods offer immediate approach for tracking or defining the chirality of the PS.
} 


\section{Acknowledgements}

I would like to thank Stefan Luther for the opportunity of working on exciting experiments and thank also my thesis committee members Tim Salditt and Martin Göpfert.

It was a great pleasure to work in a friendly group with many discussions both about science and of course non-scientific things. For the scientific discussions and collaborations I would like to especially thank Alexander Schlemmer, Philip Bittihn, Tariq Baig, and Ulrich Parlitz. For his advise and freely sharing his knowledge, I would like to thank Christian Bollensdorff.

Much thanks goes to all of those working in and around the lab. Especially, Daniel Hornung who was always there to make sure that the experiments kept running and the setup working. I thank Marion Kunze and Claudia Richter for all their work in preparing and organizing the experiments. I also would like to thank all of the group members with whom I have sat together in the lab for many hours. A list that includes almost all of the group: Alexander Schlemmer, Annette Witt, Baltasar Rüchardt, Carolin Schürer, Filippo Cosi, Henrik tom Wörden, Jan Christoph, Sayedeh Hussaini, Tariq Baig, Thomas Lilienkamp, Vineesh Kappadan, and probably more I forgot. I thank Johannes Schröder-Schetelig for keeping the cameras rolling and Laura Diaz and Andreas Barthel for building all those nice electronics.

Finally, I would like to thank everyone for their support, especially during the last bit of writing. I also have to apologize to all of those confused by the use of Canadian spelling in this thesis or stumbling over confusing structure.

This research has received funding from the German Federal Ministry of Education and Research (BMBF) (project FKZ 031A147, GO-Bio). 